\title{
Abstracts from \\ The American Telemedicine Association 2016 Annual Meeting and Trade Show
}

\author{
Jointly provided by
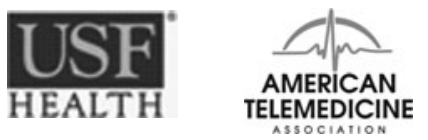 \\ May 14-17, 2016 \\ Minneapolis Convention Center \\ Minneapolis, MN
}

Please note that abstract numbering is not consecutive.

There are no missing abstracts. 


\section{Oral Presentations Abstracts}

\section{The American Telemedicine Association 2016 Annual \\ Meeting and Trade Show}

\section{Track: Direct to Consumer Room 101 JI}

\section{May 15-17, 2016 Minneapolis Convention Center SUNDAY, MAY 15, 2016}

12:00 PM-1:00 PM

Sunday, May 15, 2016

\section{Session 1 Session Title: ONLINE CARE MEASUREMENTS AND GUIDANCE}

MODERATOR: Richard J. Boxer, MD, FACS, Chief Medical Officer WellVia, Los Angeles, CA

TELEMEDICINE FOR ACUTE CARE: COMPARING IN-PERSON VISITS TO TELEMEDICINE VISITS AT A PEDIATRIC EMERGENCY DEPARTMENT

PRESENTER: Mordechai D. Raskas, MD, EdM, Pediatric Emergency Fellow, David Mathison, MD, MBA

Children's National Medical Center, Washington, DC

Introduction: Direct-to-consumer (DTC) telemedicine has the potential to improve population health and decrease health costs by minimizing expensive resources and emergency department (ED) overcrowding. However, little is known about the precision of diagnosis and treatment via DTC telemedicine from an acute care pediatric setting. Since rashes are often low acuity, without the need for lab or radiology testing, they are an ideal cohort to study. This study further evaluates the potential of DTC, real-time, video telemedicine by comparing telemedicine visits to in-person encounters for pediatric patients who seek emergency care for rashes.

Methods: We prospectively enrolled 40 patients, ages 3 months to 18 years, with a chief complaint of "rash" who presented to an urban academic pediatric ED between February and August 2015. Patients were enrolled as a convenience sample, 9am-11pm daily, including weekends. Patients were placed in private rooms and provided with smartphones with a HIPPA secure video chat to simulate a home or mobile environment. The physicians were pediatricians, pediatric emergency fellows, and pediatric emergency attendees, all of whom routinely work in the ED and for this study were initially stationed remotely with a tablet. Following the telemedicine visit, physicians completed a study form detailing the diagnosis and treatment plan. The same physician then entered the room to complete an in-person encounter with closer physical exam, including touch. The diagnoses and treatments were compared using a modified version of a previously published tool (McConnochie KM, 2006). Comparisons were categorized as "equivalent", "slight difference", or "major difference." Based on previous work, we hypothesized a 90\% concordance, and powered our sample size to be 40 patients using a 10\% error margin and 95\% confidence interval. The primary outcome was treatment concordance ("equivalent" or "slight difference") between telemedicine and in-person visits. Secondary outcomes included diagnosis concordance and survey results of patient and physician perspectives on telemedicine. The study was IRB approved.

Results: When comparing telemedicine and in-person visits, 38 of 40 (95\%) demonstrated treatment concordance. The major differences included one telemedicine visit which missed a diagnosis of scabies and another that missed scarlet fever; permethrin and penicillin, respectively, were prescribed after the in-person visits. For diagnosis, 37 of 40 visits $(92.5 \%)$ had concordance. The major differences included the above two cases. In a third case, telemedicine diagnosed contact dermatitis and the in-person visit added the diagnoses of eczema and popsicle panniculitis; there was no significant treatment change. All patients were discharged home. Telemedicine visits were preferred by 77\% of patients. When asked regarding a future implementation of a similar telemedicine model, 19\% of physicians reported that they "strongly favor" it, 58\% reported "somewhat favor", and 19\% reported "neutral". Qualitative feedback from physicians included that they felt the video connection to be poor especially when the smartphone was moved quickly. Physicians also reported a lower confidence in their diagnosis in the telemedicine visit compared with the in-person.

Conclusions: Pediatric emergency physicians can diagnose and treat rashes in a simulated DTC visit. This is one of the first studies to simulate and evaluate the physician's diagnosis and treatment choices in the pediatric emergency setting. If this tool is an effective modality with patients who had sought emergency care, there is certainly broader application for routine rash care, especially for monitoring improvement and trend following an initial encounter. In addition to providing evidence for the safety of telemedicine treating low acuity pediatric rashes, this study outlines a methodology that can easily be reproduced for a wide variety of diagnoses and complaints. Patients reported preferring telemedicine visits and physicians "somewhat favor" a telemedicine approach. These results provide further support to telemedicine, including urban settings and large academic centers. Limitations of this study include being a small study at a single site. Second, patients had already been triaged by a nurse and were included only if their chief complaint was "rash" alone. In addition, the DTC aspect was only simulated, as the patients were in emergency rooms, which, for instance, have excellent overhead lighting. On the other hand, given the environment, some parents had difficulty keeping their young children focused long enough to allow a quality video transmission of the rash. A significant limitation of this study is the use of the same physician for both the telemedicine and in-person visits. We deliberately chose this strategy, while recognizing its limitations, given that little data exists on the concordance of treatment choices for rashes between different physicians. While there is a theoretical, even unconscious, bias for a physician to anchor on an initial diagnosis, this was not found in a prior similar study (Heffner, 2009). Future studies should address this anchoring bias by including a larger sample size and utilizing two different physicians. In addition, future studies can utilize this methodology to study a wide variety of urgent care clinical problems.

\section{REFERENCES}

1. Heffner VA, Lyon VB, Brousseau DC, Holland KE, Yen K. (2009). Store-andforward teledermatology versus in-person visits: a comparison in pediatric teledermatology clinic. J Am Acad Dermatol 2009; 60(6):956-61.

2. McConnochie KM, Conners GP, Brayer AF, Goepp J, Herendeen NE, Wood NE, et al. (2006). Differences in diagnosis and treatment using telemedicine versus inperson evaluation of acute illness. Ambul Pediatr 2006;6(4):187-195; discussion 196-187.

3. McConnochie KM, Ronis SD, Wood NE, Ng PK. Effectiveness and Safety of Acute Care Telemedicine for Children with Regular and Special Healthcare Needs. Telemed J E Health 2015; 21(8):611-21. 


\section{ORAL PRESENTATIONS ABSTRACTS}

\section{Objectives}

1. Assess the accuracy of both diagnosis and treatment for direct-toconsumer telemedicine by comparing real-time video televisits with inperson encounters

2. Describe patient and pediatric emergency physician perspectives on the use of direct-to-consumer telemedicine

3. Explore the feasibility of using direct-to-consumer telemedicine for acute care from a tertiary emergency department

SINUSITIS TREATMENT GUIDELINE ADHERENCE IN THE E-VISIT SETTING: A PERFORMANCE IMPROVEMENT PROJECT

PRESENTER: Kevin L. Smith, DNP, FNP, FAANP, Chief Clinical Officer Zipnosis, Minneapolis, MN

Background: Studies demonstrate poor guideline adherence by healthcare providers for the treatment of upper respiratory infections, particularly acute bacterial rhinosinusitis (ABRS), in the appropriate prescribing of antibiotic medications. The Centers for Disease Control and Prevention estimates that nearly $50 \%$ of antibiotics prescribed in the outpatient setting are unnecessary and that overuse of antibiotics is resulting in an increase of antibioticresistant organisms. Many studies have been performed and educational campaigns implemented targeting antibiotic prescribing practices, but no long-term reduction in antibiotic prescribing has been accomplished.

Objective: The purpose of this quality improvement project was to evaluate the effect of implementing interventions for improving adherence to a clinical practice guideline for the management of ABRS for patients treated in the e-visit setting. Interventions included: providing a report to providers of their adherence rates to a ABRS clinical guideline prior to the intervention, providing updated education on the ABRS guideline, and implementing a clinical decision support system reminder within the Web-based electronic health record documentation tools.

Methods: A pre and post intervention evaluation design was used. Data were obtained from a retrospective electronic health record (EHR) data extract of all 316 diagnoses for ABRS in the pre-intervention 2-month time period and all 368 diagnoses of ABRS in the post-intervention 2-month time period. A review of the structured clinical data elements was performed to determine whether the provider adhered to the clinical guidelines, meaning that only patients meeting the criteria for ABRS were to receive an antibiotic prescription.

Results and Conclusion: The interventions resulted in a 3.3\% improvement in adherence to the ABRS clinical guideline from 95.25\% adherence preintervention to $98.4 \%$ post-intervention. These results demonstrated that the use of an educational intervention and clinical decision support resulted in improved adherence to the ABRS clinical guideline in the e-visit setting. The implications for practice could be significant in that these quality improvement interventions improve guideline adherence and reduce unnecessary prescribing of antibiotics.

\section{REFERENCES}

1. Castillo RS, Kelemen A. Considerations for a successful clinical decision support system. Comput Inform Nurs 2013; 31(7):319-26.

2. Chow AW, Benninger MS, Brook I, et al. IDSA clinical practice guideline for acute bacterial rhinosinusitis in children and adults. Clin Infect Dis 2012; 54(8):e72-e112.

3. Mehrotra A, Paone S, Martich GD, Albert SM, Shevchik GJ. A comparison of care at e-visits and physician office visits for sinusitis and urinary tract infection. JAMA Intern Med 2013; 173(1):72-4.

4. Castillo RS, Kelemen A. Considerations for a successful clinical decision support system. Comput Inform Nurs 2013; 31(7):319-26.

5. Chow AW, Benninger MS, Brook I, et al. IDSA clinical practice guideline for acute bacterial rhinosinusitis in children and adults. Clin Infect Di. 2012; 54(8):e72-e112.
6. Mehrotra A, Paone S, Martich GD, Albert SM, Shevchik GJ. A comparison of care at e-visits and physician office visits for sinusitis and urinary tract infection. JAMA Intern Med 2013; 173(1):72-4.

Objectives

1. Describe the problem of poor clinical guideline adherence in the management of upper respiratory infections resulting in overuse of antibiotics

2. Identify strategies for improving provider adherence to an acute bacterial rhinosinusitis clinical-practice guideline in the e-visit setting

3. Discuss clinical decision support strategies for improving the quality of care in the e-visit setting

A MATCHED COHORT STUDY OF PATIENTS USING URGENT CARE TELEMEDICINE AND TRADITIONAL BRICK AND MORTAR PRACTICE

PRESENTER: Laura Ten Eyck, PhD, Associate Director of Healthcare Analytics

Optum, Minneapolis, MN

Introduction: Urgent Care Telemedicine has continued to grow at a rapid pace in the United States and is gaining more visibility in the mainstream market as well. Americans are becoming more technologically savvy and are increasingly reliant on their smartphones and computers to manage many aspects of their lives. Telemedicine offers people the opportunity to address common medical issues in an efficient, timely and professional manner, using their smartphones, computers and other devices. As telemedicine services increase in number and more people utilize such services, it is important to assess their impact on patient outcomes. Additionally, it is important to assess how telemedicine compares to traditional brick and mortar medical practices. The present study was designed to compare patients who used urgent care telemedicine to a matched cohort of patients who used traditional brick and mortar care on several key outcomes including follow-up rates for the same condition, post visit medical costs, and prescription fill rates. Our hypothesis was that patients who accessed direct-topatient urgent care telemedicine would have lower follow-up rates for the same condition, lower post-index medical costs, and similar encounter-related prescription fill rates when compared to those patients who accessed brick and mortar care for conditions that fall within the scope of NowClinic.

Methods: We conducted a retrospective matched cohort study on members of a large national health plan who accessed the NowClinic direct-to-patient online care platform during calendar year 2014. Control members were selected from the same health plan's commercial population. Inclusion criteria for both groups included medical health plan eligibility and continuous enrollment in the six month pre-index and 30 day post-index period. A subset of both groups also had pharmacy benefit coverage provided by the health plan, which was required to meet the same criteria as medical health plan eligibility. The date of each NowClinic encounter in 2014 served as the index date for target group members and the date of the NowClinic appropriate urgent care, emergency department, or office visit in 2014 served as the index date for control group members. Control members were matched to target group members via propensity score matching. Logistic regression was used to create a propensity score model based on 39 baseline variables including member level demographics, index encounter characteristics, pre-period spend, chronic medical conditions, pharmacy benefit coverage and prescription utilization. Final 1:1 matching resulted in a sample of 1,132 NowClinic patient encounters with 1,132 matched controls from brick and mortar practice settings.

Results: NowClinic users were significantly less likely to complete a followup visit for the same diagnosis within 15 days of the index event when compared to their matched controls ( $1.8 \%$ versus $3.9 \%, p=0.003)$. The same pattern of results occurred within 30 days following the index event, where NowClinic users were significant less likely to complete a follow-up visit for the same diagnosis than were their matched controls $(2.5 \%$ versus $5.2 \%$, $p=0.0009$. NowClinic users also demonstrated significantly lower post-index 
allowed medical costs than their matched controls at 15 days ( $\$ 83.42$ versus $\$ 110.28, \mathrm{p}<0.0001)$ and 30 days $(\$ 218.59$ versus $\$ 257.93, \mathrm{p}=0.0159)$. NowClinic users with valid health plan pharmacy coverage filled at least one prescription within one day of their index event at rates similar to those in the matched control group (70.5\% versus 66.8\%, $\mathrm{p}=0.28$ ).

Conclusions: NowClinic users were less likely to have a follow-up visit for the same condition, incurred lower post-index medical costs at 15 days and 30 days, and had nearly identical rates of encounter-related prescription fills when compared to the matched control group. Such outcomes demonstrate that for appropriate treatment conditions, direct-to-patient telemedicine services are a viable alternative to traditional brick and mortar practice-based services.

\section{Objectives}

1. Compare follow-up rates for urgent care telemedicine and traditional brick and mortar practice patients

2. Describe the impact that urgent care telemedicine has on patients' postvisit medical costs

3. Demonstrate that urgent care telemedicine patients fill encounter-related prescriptions at similar rates when compared to traditional brick and mortar practice patients

\section{SUNDAY, MAY 15, 2016}

\section{1:15 PM-2:15 PM Sunday, May 15, 2016}

\section{Session 9 \\ Session Title: FINANCIAL CONSIDERATIONS OF YOUR HEALTH SYSTEM'S DIRECT-TO-CONSUMER TELEMEDICINE PROGRAM}

PRESENTER: Mathew Hanley, MBA, Director of Strategic Affiliations OSF Healthcare, Peoria, IL

PRESENTER: Matt Thorne, BA, Chief Operating Officer/ Vice President Finance

Carena, Seattle, WA

Launching a telemedicine program for a health system can be a daunting task. Should you buy or build your solution? What will the operational impact be for implementation as well as for sustaining the program? How will your provider group react to a new offering? Undoubtedly, you will encounter the question of how much it will cost and how will the system measure the economic impact and value? The list of questions could go on.

OSF HealthCare in Peoria, Illinois found themselves asking these questions in 2013. They wanted a direct-to-consumer telemedicine offering to provide virtual care to their existing patients and expand their reach to new patients. Over the course of a year, OSF evaluated whether to buy or build by modeling the financial impact and ROI of each option in both the near and the longterm. Ultimately, they decided to buy rather than build for both financial and non-financial reasons. Now, 11 months after launching OSF OnCall, the service is available to 1.3 million patients in central Illinois.

In this session, you'll hear from Mathew Hanley, Director of Strategic Affiliations at OSF, and Matt Thorne, COO of Carena. They will discuss the key questions to ask when contemplating a telemedicine solution, considerations when answering those key questions, as well as who needs to be at the table when having those discussions. They will also provide a framework for an economic analysis of a direct-to-consumer telemedicine offering broken out into three categories:

- Financial and economic measures

- Intrinsic value with some correlation to financial measures

- Intrinsic value without a direct correlation to financial measures
Whether your health system is contemplating a telemedicine solution or you're still on the sidelines, this session will give you the tools and insights for a successful process.

\section{Objectives}

1. Provide a framework for modeling and analyzing the ROI of a directto-consumer telemedicine program

2. Outline considerations for a health system when deciding whether to buy or build their telemedicine program

3. Explain the importance of finding a model that works best for clinical and business objectives of a health system

\section{MONDAY, MAY 16, 2016}

8:00 AM-8:45 AM Monday, May 16, 2016

\section{Session 17 \\ Session Title: RETAIL KIOSKS IN URGENT CARE CLINICS: WHY IT'S THE NEW NORMAL}

MODERATOR: Matt Levi, MPH, MHA, Director of Virtual Health Services Franciscan Health System, Tacoma, WA

PRESENTER: Lana Adzhigirey, RN, MN, CPHQ, Program Manager Care Transformation

Virtual Health Services, Tacoma, WA

PRESENTER: Korina Tanner, MD, Medical Director of Urgent Care Services CHI Franciscan Health, Tacoma, WA

Currently, the access issues we have been experiencing in Emergency Departments have spread to the less emergent Urgent Care clinics. As the volumes at these clinics steadily increased, the wait times have gotten longer in some cases over two hours. Our operating hypothesis included the idea that introducing a Virtual Urgent Care kiosk at an Urgent Care Clinic will decrease the wait times at the Urgent Care Clinic and increase patient satisfaction.

Our findings included some surprises such as not being able to use the existing exam rooms as we hoped. We also found this case to break down the biggest barrier of buy in from the executive team which resulted in the approval of Virtual Urgent Care dedicated providers. We have yet to finalize the patient perception and will share the results in the presentation.

In conclusion, access to care is a pressing dilemma which is not going away. As the Virtual Urgent Care is becoming the standard of care, our organization has invested in implementing a kiosk and created a new standard of providing urgent care: via different modes of access which can save our patients hours in wait time.

\section{REFERENCES}

1. Berwick DM, Nolan TW, Whittington J. The triple aim: care, health and cost. Health Affairs 2008;27(3):759-69.

2. O'Malley AS. After-hours access to primary care practices linked with lower emergency department use and less unmet medical need. Health Affairs 2013; 32(1):175-83.

\section{Objectives}

1. Describe the current state of Urgent Care and issues clinicians are facing today

2. Describe the decision making process to merge the physical and virtual services in one clinic

3. Share lessons learned and results of implementing a Virtual Urgent Care kiosk at a physical clinic location 
MONDAY, MAY 16, 2016

11:00 AM-12:00 PM
Monday, May 16, 2016
TUESDAY, MAY 17, 2106

11:00 AM-12:00 PM

Tuesday, May 17, 2016

\section{Session 25 \\ Session Title: ASSIMILATION OF VIRTUAL VISITS TO THE HEALTHCARE SYSTEM: KEEPING QUALITY AT THE FOREFRONT}

MODERATOR: Karen K. Scott, Senior Director - Marketing, Product and Innovation - Telehealth and Onsite Initiatives

UnitedHealthcare, Minnetonka, MN

PRESENTER: Karen K. Scott, Senior Director - Marketing, Product and Innovation - Telehealth and Onsite Initiatives

UnitedHealthcare, Minnetonka, MN

PRESENTER: Michelle Lobe, MA, MBA, Vice President, Network Strategy and Innovation

UnitedHealthcare, Chicago, IL

PRESENTER: Roy Schoenberg, MD, MPH, Chief Executive Officer American Well, Boston, MA

PRESENTER: Ian Tong, MD, Chief Medical Officer

Doctors on Demand, San Francisco, CA

Market changes (ATA accreditation program; AMA telemedicine policy, etc.) and intersection of telehealth segments (Video Telemedicine; Remote monitoring; and m-Health) open the door to continued adoption, growth and innovation of virtual visits. The healthcare industry must be vigilant in ensuring patient experience and quality care remain at the forefront. This can be accomplished through delivering consistency with flexibility around technology, credentialing and billing standards. This case study explores advancing virtual visits beyond application-specific integrations to full assimilation across the care delivery system, as a modality that will support the Triple Aim fully across all patients and populations. Positioning virtual visits as a beneficial, common and widely accepted aspect of patient healthcare will break down not just geographic barriers, but also lifestyle and access-to-care barriers. This will involve input from all the parties involved: from regulatory bodies, to professional organizations, to patients and their advocates. Research findings include: cost-effectiveness; ease of use; time-savings; healthcare availability access for patients in rural and under-served areas; and opportunities to expand practices and serve a wider audience.

Objectives

1. Discuss Virtual Visit model specific to patient choice; quality assurances; and multi-point integration

2. Discuss need for benefit coverage, designs and networks of virtual visit provider groups that will let consumers see and talk to a doctor, obtain a diagnosis and a prescription, all from their computer, tablet or mobile phone

3. Discuss need for standards for network providers based on American Medical Association and Federation of State Medical Boards guidelines, and how regulatory environment will factor into the telehealth arena

\section{Session 46 \\ Session Title: ATA ACCREDITATION OF ONLINE PATIENT CONSULTATIONS}

MODERATOR: Edward M. Brown, MD, Chief Executive Officer Ontario Telehealth Network, Ontario, Canada

PRESENTER: Michael J. Ackerman, PhD, ATA Commissioner and Retired Assistant Director

High Performance Computing and Communications, National Library of Medicine, Bethesda, MD

PRESENTER: Keith Algozzine, PA-C, CEO

Upstate Concierge Medicine, Clifton Park, NY

PRESENTER: Ian Tong, MD, Chief Medical Officer

Doctor on Demand, San Francisco, CA

The importance of quality and safety cannot be overemphasized in relation to the proliferation of online patient healthcare providers and clinical services delivered in the United States. Critical perspectives about the importance and value of healthcare accreditation are explored by panelists who are involved with the ATA Accreditation Program for direct-to-consumer Online Patient Consultations, including a sitting ATA Commissioner and representatives from accredited Organizations. The session is designed to connect the "dots" and other salient considerations about "quality" for providers, payers, and regulators operating in the telemedicine space.

Objectives

1. Understand current direct to consumer market and the value and role of accreditation in quality healthcare delivery and consumer safety.

2. Gain an understanding of the ATA accreditation program.

3. Learn about the accreditation process from accredited organizations, an ATA commissioner and an organization currently going through the survey process.

\section{TUESDAY, MAY 17, 2016}

\section{1:15 PM-2:15 PM}

Tuesday, May 17, 2016

\section{Session 54}

\section{Session Title: PROVEN ROI OF VIRTUAL VISITS TO IMPROVE CONVENIENCE AND ACCESS TO QUALITY CARE}

MODERATOR: Henry DePhillips, MD, Chief Medical Officer

Teladoc, Nashville, TX

PRESENTER: Henry DePhillips, MD, Chief Medical Officer

Teladoc, Nashville, TX

Telehealth is the most rapidly growing sector in the healthcare industry. Virtual doctor visits offer a proven solution to address many of the challenges facing U.S. employers, health plans and consumers today, including skyrocketing costs, doctor shortages, long wait times, absenteeism and lost productivity, to name a few. There is a rapidly growing demand for telehealth solutions to reduce the rising cost of healthcare benefits, which is estimated at approximately $\$ 620$ billion annually. Telehealth successfully delivers the diagnosis and treatment of common, uncomplicated conditions remotely, along with prescribing when necessary. This translates to clinical resolution quickly, easily and affordably. Telehealth reduces unnecessary visits to ER and urgent care, saving time and money. 
Telehealth provides $24 / 7$ access to a doctor, by phone or video, from any location. Large employers have recognized the value. In fact, $74 \%$ of large employers plan to offer telehealth services in 2016.

This presentation will feature the telehealth program results Reynolds American, the parent company of R. J. Reynolds Tobacco Company. We will explore their 12-month utilization data showing the effective redirection of care from less efficient modalities across its employee base of approximately 7,100 , with total membership of approximately 14,200 . The ROI savings data is claims-based and compares telehealth costs to traditional care.

We will evaluate the short-term spending and resource utilization of members who used Teladoc compared to those who chose to receive care for similar conditions in a physician's office or ER. The results summary indicates a savings of direct medical costs per visit of approximately $\$ 253$ per episode ( $\$ 191$ savings per episode redirected from an office and $\$ 2,661$ per episode redirected from the ER). Using actual Reynolds member-reported redirection, the group has saved nearly $\$ 181,000$ year-to-date. The overall annualized savings is expected to reach $\$ 271,000$, representing a utilization rate of $19 \%$ (*based on YTD analytics. Numbers are subject to slight $+/$ - fluctuation.) In addition, Reynolds will also demonstrate that employee productivity savings were equally significant, estimated to be an additional savings of $\$ 62,000$ based on time lost from work.

Reynolds will provide factual narrative around their member engagement, revealing that utilization is strongly influenced by seasonality and well-timed communication campaigns help to increase awareness, behavior change and savings. Their users rated their virtual doctor visit service at $91 \%$ and $90 \%$ of members surveyed said they would use the service again. This presentation will also address the key elements of an effective deployment, communication and member engagement strategy, to achieve the maximum ROI. We'll also demonstrate how we apply predictive modeling and other advanced analytic tools to better understand variables that impact utilization, including member personas, age, gender, industry, health risk status and even past engagement as predictors for future use.

\section{Objectives}

1. Exhibit the benefits of telehealth for increasing access, lowering cost and improving outcomes while impacting savings for organizations through measurable ROI

2. Analyze claim based resolution rates and costs of virtual visits compared to traditional care centers

3. Identify key drivers of program success, including member engagement, utilization and increased ROI potential year over year

\section{TUESDAY, MAY 17, 2016}

\section{3:00 PM-4:00 PM}

Tuesday, May 17, 2016

\section{Session 62}

\section{Session Title: DIRECT TO EMPLOYER TELEHEALTH}

MODERATOR: Jeffrey Benabio, MD, Physician Director of Healthcare Transformation

Kaiser Permanente, San Diego, CA

\section{IMPLEMENTATION OF A DIRECT TO EMPLOYER TELEHEALTH CLINIC}

PRESENTER: Tim Sagers, MD, Medical Director, Business Health Solutions Mercy Physician Services, Cedar Rapids, IA

Background: Healthcare consumer behavior is rapidly evolving. Hastened by technology driven expectations and drastic health benefit cost shifts, patients are looking for new access models for lower cost healthcare services. At the same time, employers of all sizes are looking for innovative ways to provide value based healthcare options for their employees to off-set cost shifting and the inevitable movement to large deductible health plans (HDHP). On-site, employer- based telemedicine offers a solution which adds value to existing employer based benefit offerings by providing convenient access to urgent and primary care services at a fraction of the cost of traditional healthcare. Although some traditional care confines are required, i.e. private space, the cost to implement an onsite telemedicine clinic can be quite attractive to employers of all sizes. Recent experiences also demonstrate very high levels of employee/patient satisfaction. Experience: In 2015, MercyCare Business Health Solutions deployed an on-site, employer based clinic in close cooperation with Rockwell Collins, a large international technology and defense corporation located in Cedar Rapids, IA. With an eligible local employee population of 4,600, Rockwell Collins was searching for a value added healthcare option for their local employees, a majority of whom are served by a HDHP. Prior to deployment, close attention was given to developing a reimbursement model which would allow for claims adjudication with Wellmark of Iowa. Communication with stakeholders in the local healthcare delivery system was also critical to successful deployment of the MercyCare/Rockwell Collins telemedicine clinic as close integration into the local primary care networks is essential. The clinic is fully equipped with all diagnostic capabilities needed to deliver episodic acute care services. Technologies from CISCO, AMD and Agnes are incorporated into the delivery model. Care delivery in the MercyCare/Rockwell clinic is based on a very deliberate menu of services, limiting liability and providing for a complete patient experience within the telemedicine space. Employees who visit are offered a flat, negotiated fee rate currently about $50 \%$ less than traditional urgent and primary care delivery. Employee/patient acceptance of the model and subsequent utilization continues to grow and the current patient satisfaction is at the 99th percentile (Press-Ganey 2015).

Conclusion: 0n-site, employer-based telemedicine provides an innovative solution to meet both consumer expectation and employer needs. Well recieved by patients, this model is a functional bridge from traditional care delivery to that of a true virtual care experience. Several key factors should be noted: 1 . Close conversation with payors to build a meaningful reimbursement model is essential. 2. Close integration and communication with the local primary care networks is mandatory. 3. Close and ongoing attention to patient satisfaction and employer/client feedback is critical to ongoing success.

\section{REFERENCES}

1. Advisory Board. Telehealth Industry Trends 2015/ppt.

2. Adans, J et al. Advisory Board. White Paper:2015 Healthcare IT Top Ten. 2015. $1-17$.

3. State Telehealth Reimbursement Policies. Center for Connected Health Policy. Sept. 2014. 1- 2014.

\section{Objectives}

1. Apply current regulatory and financial factors in the implementation of a payor based reimbursement model for on-site telemedicine clinics

2. Demonstrate the importance of communication and education in the transformation of the existing healthcare delivery model to one based on telemedicine capability

3. Identify factors critical to the succesful deployment and operation of an on-site employer based telemedicine clinic

\section{TUESDAY, MAY 17, 2016}

4:15 PM-5:15 PM Tuesday, May 17, 2016

\section{Session 70 Session Title: URGENT CARE TELEHEALTH: CASE REVIEWS}

PRESENTER: Lo Fu Tan, MD, MS, Associate Medical Director, On Demand Medicine

Southwest Medical Associates, Optum Nevada, Las Vegas, NV 


\section{ORAL PRESENTATIONS ABSTRACTS}

Urgent Care Telehealth is here but is missing Clinical Quality Assessment tools and guidelines. There is little or no literature that looks at the topic. Programs have had to devise their own "Quality Assurance" programs. We wish to present one part of ours through case presentations as a way to illustrate important quality points that have been missed during visits.

We have several case presentations drawn from 10,000 Urgent Care Telehealth visits over a 2 year period. These have been recorded in mock audiovisual format. Each will be shown to the audience, and an ongoing discussion, question and answer period will ensue to help determine quality issues. Ways to improve future clinical care will be determined in the same interactive format.

Once all cases are completed, we will highlight Quality points under the following areas: Clinical, Organizational, and Legislative.

Finally, we will present a way to assess the effectiveness of this case-based quality review tool, namely by doing follow-up case reviews.

\section{Objectives}

1. Present cases to illustrate some quality problems

2. Discuss quality criteria based on clinical guidelines, organizational policies, and legislative requirements

3. Discuss quality monitoring of Urgent Care Telehealth

\section{Track: Acute Care Room 102 C}

\section{SUNDAY, MAY 15, 2016}

\section{Session 2 Session Title: UNIVERSITY OF VIRGINIA PILOTS TELESTROKE PROGRAM}

MODERATOR: David Cattell-Gordon, MDiv, MSW, Director of UVA

Telemedicine and Rural Network Development

University of Virginia Office of Telemedicine, Charlottesville, VA

PRESENTER: Nina Solenski, MD, Associate Professor of Neurology UVA Primary Stroke Center, Charlottesville, VA

PRESENTER: Brian Gunnell, Senior System Engineer

University of Virginia Health System, Charlottesville, VA

Virginia hospitals, similar to other states with substantial rural communities, have faced several challenges that prevent rural hospitals from providing specialty medical care to patients in need. To combat this access to care issue, the University of Virginia (UVA)'s Center for Telehealth transformed patient delivery through technology-driven telemedicine programs throughout the state. UVA's Center for Telehealth was developed so that medical fellows, doctors and specialists can connect virtually with patients using solutions like immersive video conferencing technology and unified communications. UVA's Center for Telehealth projects currently includes a 125-site network in Virginia and the center has conducted more than 40,000 patient encounters in 40 sub-specialties of medicine, saving patients roughly 9.2 million miles of travel in healthcare support.

One of these exemplary projects is the telestroke program.

Nearly 800,000 people in the U.S. have a stroke each year, and this estimate is projected to rise to 1.5 million by the year 2050. Currently, strokes are the fourth leading cause of death and a leading cause of long-term adult disability in the U.S. and around the world. An analysis, conducted by UVA, of 46 U.S. rural hospitals, including 4,462 teleneurology emergency department (ED) consults, demonstrated rapid response time for a teleconsultation with a neurologist once the physician was alerted of the potential stroke patient. However, time from a patient's ED arrival, to the appropriate treatment of the patient, did not meet current American Stroke Association benchmarks of less than 60 minutes. Barriers for treatment can include initial triage, recognition of atypical stroke presentations, bedside instruction on the neurology exam and preparation delay of TPA.

UVA implemented its enhanced telestroke program at critical access hospitals in Bath and Culpeper, Virginia. UVA's program utilizes interactive, high-definition and real-time videoconferencing to enable effective, scalable and easy-to-manage deployments anywhere, on any device or hospital cart. This effort provides access to stroke neurology services for patients in one of the most rural regions of Virginia and has led to an expansion of telestroke services throughout the Commonwealth. At telestroke hospitals, medical staff also use hospital carts with videoconferencing end-points, which are ready 24 hours a day for potential stoke victims. Neurologists from UVA's Center for Telehealth are equipped with end-points in their homes, in fixed and mobile units, as well as their medical centers. This technology enables the neurologists to administer life-saving assessments in a fraction of the time that would be needed to transport a patient to a primary stroke center. Adding telestroke allows a small, rural hospital to become as effective as a primary stroke center in its rate of TPA administration, which is very significant in terms of morbidity and quality of life.

UVA's telestroke program also incorporates educating healthcare professionals at any level to become more skilled in stroke assessment and treatment. The Stroke Telemedicine and Tele-education (STAT) program includes training at all levels of the acute stroke care continuum: EMS and ED physician training, stroke specific telepresenter training which instructs medical and physician assistants on ways administer physical exams on patients to support off-site neurologists, and a checklist protocol to standardize the evaluation and treatment of the stroke patient. The Southside Telehealth Training Academy and Resource Center (STAR), a joint program sponsored by UVA's Center for Telehealth and the New College Institute, is a premier training program for healthcare providers seeking to use advanced telemedicine and telehealth systems to improve access to quality healthcare for rural and medically-underserved populations. To date, this is the largest cohort of rural telestroke patients analyzed and the stroke specific online telepresenter is the first of its kind.

As the practice of telestroke becomes more mainstream, UVA's investigators are now developing novel uses of mobile telemedicine to improve acute neurological care. Examples include a tablet-based system for rapid assessment of stroke patients during ambulance transport (iTREAT Study) and using wearable devices to better evaluate stroke patients during after-hours consultation (NeuroEGG Study).

With the ability to access specialists on-demand, the program is maximizing patient recovery by delivering a timely assessment, diagnosis and determination of eligibility for short-term therapy and treatment. Due to comprehensive telestroke programs such as this, TPA administration across rural hospitals in Virginia has risen. UVA's telestroke program is looking to create a standards-based operating system for stroke care in healthcare facilities across the nation.

\section{REFERENCES}

1. Lippman JM et al. Mobile telestroke during ambulance transport is feasible in a rural EMS setting: the iTREAT Study. Telemed J E Health ePub - Nov 24, 2015.

2. Padrick MM, Chapman Smith SN, McMurry TL, et al. Abstract 90: NIH Stroke Scale Assessment via iPad-based Mobile Telestroke During Ambulance Transport is Feasible - Pilot data from the Improving Treatment with Rapid Evaluation of Acute Stroke via Mobile Telemedecine (iTREAT) Study. Stroke; a journal of cerebral circulation 2015;46:A90

3. Chapman S, Lippman J, Gunnell B, et al. Mobile Telestroke During Ambulance Transport Is Feasible in a Rural EMS Setting - Pilot Data From The Improving Treatment With Rapid Evaluation Of Acute Stroke Via Mobile Telemedicine (iTREAT) Study (S5.002). Neurology 2014; 82:S5.002.

4. Ali SF, Viswanathan A, Singhal AB, Rost NS, Forducey PG, Davis LW, Schindler J, Likosky W, Schlegel S, Solenski N, Schwamm LH; Partners Telestroke Network The TeleStroke mimic (TM)-score: a prediction rule for identifying stroke mimics evaluated in a Telestroke Network. J Am Heart Assoc 2014; 23(3):e000838. 


\section{Objectives}

1. Apply and understand principles of the role of bedside telepresenter for stroke specific patients, and the benefit of tele-education

2. Demonstrate understanding of the technical challenges in using mobile telestroke platform

3. Describe the data and implication of time to treatment for TPA in rural U.S. hospital

\section{SUNDAY, MAY 15, 2016}

1:15 PM-2:15 PM Sunday, May 15, 2016

\section{Session 10 \\ Session Title: USING TELEHEALTH TO FACILITATE BUNDLED PAYMENTS IN A STROKE SYSTEM OF CARE}

MODERATOR: Kenneth Gaines, MD, Vascular Neurologist

Ochsner Medical Center, New Orleans, LA

PRESENTER: Kenneth Gaines, MD, Vascular Neurologist

Ochsner Medical Center, New Orleans, LA

PRESENTER: Patricia Commiskey, DrPH, MA, Clinical Research Scientist Vanderbilt University Medical Center, Nashville TN

Introduction: Stroke is the 5th leading cause of death in the United States and requires timely intervention at onset and chronic care post-stroke to decrease morbidity and mortality. Stroke patients move through four distinct Nodes of Care, from initial acute assessment and diagnosis through in-hospital treatment to subacute care at a rehabilitation unit or skilled nursing facility or chronic care at home to reduce recurrence through risk factor reduction. Current stroke care systems are poorly coordinated and foster confusion, as patients and families face conflicting geographic care delivery locations and constantly changing providers. Therapeutic/prognostic recommendations and health-related education often conflict from one Node to another because of differing specialty focus and limited information from prior Nodes. Better designed systems that integrate care and account for these Node transitions are needed. Telemedicine is a powerful tool that can be utilized to foster coordinated care and improve outcomes. Telestroke has been applied to increase time to treatment and can be cost effective, as research has shown that using intravenous recombinant tissue-type plasminogen activator (rtPA) in an ischemic stroke patient in the first three hours results in a 76\% greater likelihood of a good or excellent outcome, saving the healthcare system $\$ 25,000$ over the lifetime of each patient treated. Given expected increases in strokerelated medical and disability costs, innovative reimbursement models like bundled payments, which provide reimbursement based on expected costs per episode rather than per visit, allow treatment for stroke patients' complex acute and chronic care needs, including preventive mechanisms to reduce recurrent stroke risk factors. Well designed bundled payment models combining evidence-based guidelines, multidisciplinary approaches, and innovative technologies like telestroke are essential to reduce costs and ensure quality care. This panel presentation will describe a novel telestroke system as a tool to facilitate integrated care and develop a comprehensive bundled payment model for stroke. Its use in an innovative stroke care redesign initiative will be highlighted, including how results from this program are being used to develop a bundled payment model.

Methods: Ochsner Clinic Foundation established a comprehensive hub and spoke telestroke system that incorporates both "drip and ship" and "drip and keep" methodology at sites across Louisiana. Ochsner was also awarded a threeyear Health Care Innovation Award (HCIA; grant \# 1C1CMS331043; PI: KGaines) from the Centers for Medicare and Medicaid Services (CMS) in 2012 to test a novel Integrated Stroke Care Delivery Model combining coordinated acute and in-hospital care with home-based care and education for 12 months postdischarge; an overarching goal was to develop a bundled care model for stroke from acute onset through one year post stroke discharge. This Integrated Stroke Care Delivery Model combined in-hospital Stroke Central with home-based, outpatient Stroke Mobile. Once discharged, patients and families were visited 12 times at home to assess overall health and recovery, evaluate risk factors, and provide comorbidity management, education, and caregiver/family support. The project described was supported by Grant No.1C1CMS331043 from the Department of Health and Human Services (US HHS), CMS. Contents of this publication are solely the responsibility of the authors and do not necessarily represent the official views of the US HSS or any of its agencies. Research presented here was conducted by the awardee, and findings might or might not be consistent with or confirmed by the independent evaluation contractor.

Results: Vascular neurologists at the primary hub hospital (Ochsner Medical Center) completed approximately 4,000 telestroke consults for ischemic, hemorrhagic, and TIAs from 2009-2014, averaging approximately 1,000 consults per year. Ischemic stroke patients receiving rtPA by 2014 increased 275\% compared to pre-telestroke rtPA utilization rates, and significant personnel efficiency was seen overall, including site management by a small group of vascular neurology specialists and neuro-intensive care, neuro-interventional, and neurosurgical physicians. In addition, 2,979 patients with suspected stroke symptoms were seen in Stroke Central from January 2013-June 2015 and 459 patients had been seen in Stroke Mobile since February 2013, with 136 completing all 12 home-based visits. Technology has been utilized throughout this initiative, and a key goal has been development of a bundled payment model. Conclusions: Telestroke programs have great potential for increasing and expediting stroke care across Nodes of Care. Additional research is needed to evaluate cost effectiveness, but Ochsner's program has furthered exploration of using technology and innovative payor models to encourage new stroke care delivery models.

\section{REFERENCES}

Reference list (19 total) for this abstract available from Submitter upon request.

\section{Objectives}

1. Describe a novel, integrated telestroke system and its potential application to facilitate a more integrated care model

2. Discuss the benefits of a bundled payment program and the applicability of telestroke as a tool to coordinate care as part of this model

3. Illustrate telestroke use and application in a bundled payment program as part of an innovative stroke care redesign initiative at Ochsner Medical Center

MONDAY, MAY 16, 2016

8:00 AM-8:45 AM

Monday, May 16, 2016

\section{Session 18 \\ Session Title: MEASURING AND IMPROVING TELESTROKE OUTCOMES}

MODERATOR: Matthews Gwynn, MD, Chief Executive Officer AcuteCare Telemedicine, Atlanta, GA

RACIAL AND GENDER DIFFERENCES IN TISSUE PLASMINOGEN ACTIVASE METRICS FOR ACUTE ISCHEMIC STROKE OVER TELEMEDICINE

PRESENTER: Amanda Jagolino, MD, Assistant Professor, Shima Bozorgui, MD, MPH, Jignesh Patel, MD, Malcolm Irani, BA, Christy Ankrom, BS, TC Cossey, MD, Anjail Sharrief, MD, MPH, Farhaan Vahidy, MD, PhD, Sean Savitz, MD, Tzu Ching Wu, MD

University of Texas Health Sciences Center Houston, Houston, TX 


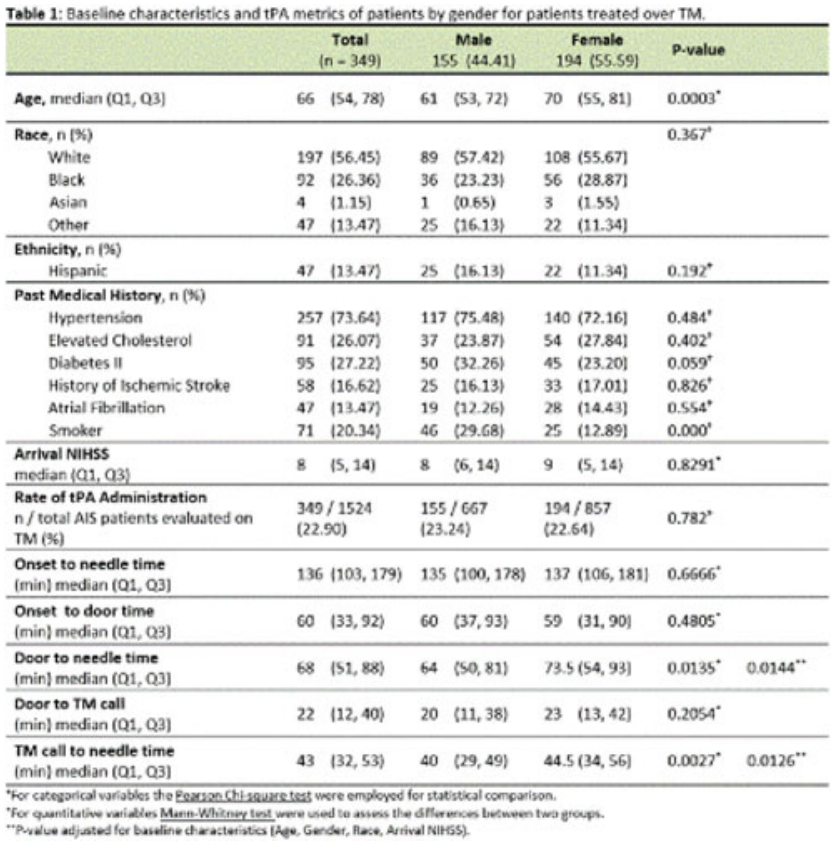

Introduction: Several studies have demonstrated racial/ethnic and gender disparities in acute ischemic stroke management which persist after adjustment for measures of socioeconomic status. As acute ischemic stroke management and intravenous (IV) tissue plasminogen activase (tPA) treatment via telemedicine increases, it is important to assess for differences in treatment metrics by race/ethnicity and gender.

Methods: We identified 349 patients in our telemedicine stroke registry who received IV tPA at one of our 14 spoke hospitals from 2/2014 to 5/2015. We abstracted baseline characteristics, and compared tPA metrics among different groups, including race/ethnicity (White, Black, Hispanic, Asian) and gender.

Results: Baseline characteristics and clinical variables are found in both tables. The median time between telemedicine code stroke alert and tPA administration was longer for women compared to men ( 44.5 vs $40, p=0.00$ ), which is reflected in a significantly longer median door to needle time for women (73 vs $64, p=0.01$ ) (Table 1 ). This difference persisted when adjusted

\begin{tabular}{|c|c|c|c|c|c|c|c|}
\hline & & $\begin{array}{c}\text { White } \\
(n=19 n)\end{array}$ & $\begin{array}{c}\text { Black } \\
(n=92)\end{array}$ & & $\begin{array}{l}\text { Hispanic } \\
(n=47)\end{array}$ & $\begin{array}{l}\text { Asian } \\
(n=4)\end{array}$ & P.value \\
\hline Age, modian (Q.1, Q3) & 70 & $(55,79)$ & $\cos (51.5,73)$ & 60 & $(48,73)$ & $71.5(59,87.5)$ & $0.0057^{\circ}$ \\
\hline $\begin{array}{c}\text { Gender, } n(5) \\
\text { Male }\end{array}$ & 89 & (45.18) & $35 \quad$ (39.13) & 25 & (53.19) & $\{25.00\}$ & $0.380^{\prime}$ \\
\hline Pest Medical History, $n[(x)]$ & & & & & & & \\
\hline Mrpertention & 141 & {$[71.57]$} & $77(83.70)$ & 30 & (63.83) & $\{100\}$ & $0.027^{\prime}$ \\
\hline Elevatac Cholesterol & 55 & (27.92) & $20 \quad(21.74)$ & 10 & (21.28) & $100 ;$ & $0.010^{\circ}$ \\
\hline Clabotos II & 42 & (21.32) & $39 \quad(42.39)$ & 12 & (23.40) & (25.08) & $0.002^{\prime}$ \\
\hline Iechemic atroko & 30 & (15.23) & 17 (18.A8) & , & (14.89) & (25.00) & $0.720^{\prime}$ \\
\hline Atrial fibenllation & 33 & (26.75) & $20 \quad(20.57)$ & 2 & $(4.26)$ & $10.00\}_{1}$ & $0.200^{\prime}$ \\
\hline Smoket & 40 & (20.30) & 22 (23.91) & 6 & $(12.7)$ & $1 \quad(25.00)$ & $0.411^{\prime}$ \\
\hline $\begin{array}{l}\text { Arrival RathSS } \\
\text { median (Q1, Q3) }\end{array}$ & 9 & $(S, 16)$ & $=(6,13.5)$ & 8 & $(5,12)$ & $23 \quad(6.5,23.5)$ & $0.47 m^{\circ}$ \\
\hline $\begin{array}{l}\text { Rate of tPA Administration } \\
\text { n/total AIS pationts } \\
\text { evaluated on TMM (F) }\end{array}$ & & $/ 613(24.32)$ & $92 / 264(25.84)$ & & $165|22.17\rangle$ & $4 / 28 \quad(12.5)$ & $0.349^{\prime}$ \\
\hline $\begin{array}{l}\text { Onset to necdie time } \\
\text { (min) median (Q1, Q3) }\end{array}$ & & $(100,170)$ & 125.5 (10:5, 196) & 142 & $(112,215)$ & $129(26.5,149)$ & $0.2814^{*}$ \\
\hline $\begin{array}{l}\text { Onset to door time } \\
\text { (min) modian (a.1, 03) }\end{array}$ & 58 & $(34,88)$ & $59.5(30,105)$ & 60 & $(42,205)$ & $58.5(29,72.5)$ & 0.7047 \\
\hline $\begin{array}{l}\text { Door to needle time } \\
\text { (min) median }(01,03)\end{array}$ & 67 & $(50,87)$ & $68.5(50,38)$ & 77 & $(53,200)$ & $70.5(57.5,76.5)$ & $0.4834^{\circ}$ \\
\hline $\begin{array}{l}\text { Door to TM call } \\
\text { (min) modian (Q1, O3) }\end{array}$ & 24 & $(12,40)$ & $19 \quad|12.5,37.5|$ & 21 & $(10,521$ & $19 \quad[16.5,30,5)$ & $0.7175^{\circ}$ \\
\hline $\begin{array}{l}\text { IIM call to needle time } \\
\text { (min) median }(01,03)\end{array}$ & 40 & $(30,52)$ & $42.5(33,57.5)$ & 45 & $(33,55)$ & $40.4(35.5,51.5)$ & $0.2651^{\circ}$ \\
\hline
\end{tabular}

for age, race, and baseline NIH Stroke Scale $(p=0.01)$. However, there was no significant difference in IV tPA metrics between race in our dataset (Table 2). Conclusions: Further investigation is needed to define factors contributing to gender difference in IV tPA treatment metrics over telemedicine.

\section{REFERENCES}

1. Bladin CF, Cadilhac DA. Effect of Telestroke on Emergent Stroke Care and Stroke Outcomes. Stroke 2014; 45(6):1876-880.

2. Boehme AK, Siegler JE, Mullen MT, et al. Racial and Gender Differences in Stroke Severity, Outcomes, and Treatment in Patients with Acute Ischemic Stroke. J Stroke Cerebrovasc Dis 2014; 23(4):e255-e261.

\section{Objectives}

1. Identify areas of improvement in healthcare disparities

2. Identify areas of improvement in acute ischemic stroke care via telemedicine

3. Apply knowledge from this subset of stroke patients to own telemedicine practice

\section{IMPROVING TRENDS IN DOOR TO NEEDLE TIME IN A STATEWIDE TELESTROKE PROGRAM}

PRESENTER: Ellen Debenham, RN, Neuroscience Telemedicine Manager, Jessica Hannah, MD, Christine Holmstedt, MD, Nancy Turner, RN Medical University of South Carolina, Charleston, SC

Background and Purpose: The goals of telestroke programs are to provide safe and efficient stroke care to hospitals. An important marker of efficient acute stroke care delivery is door-to-needle (DTN) time, the time it takes from entrance into the emergency department (ED) to administration of alteplase. We sought to determine if our interventions were improving DTN time within our statewide telestroke network.

Methods: From 5/4/2008 - 7/12/2015, we prospectively collected data on DTN time from consultations performed in our 18-hospital statewide telestroke network. DTN time was recorded on 765 of the 4985 consults where alteplase recommendations were documented. For all active hospital sites combined, the yearly mean DTN time was determined and compared by year with a one-way ANOVA and Tukey test.

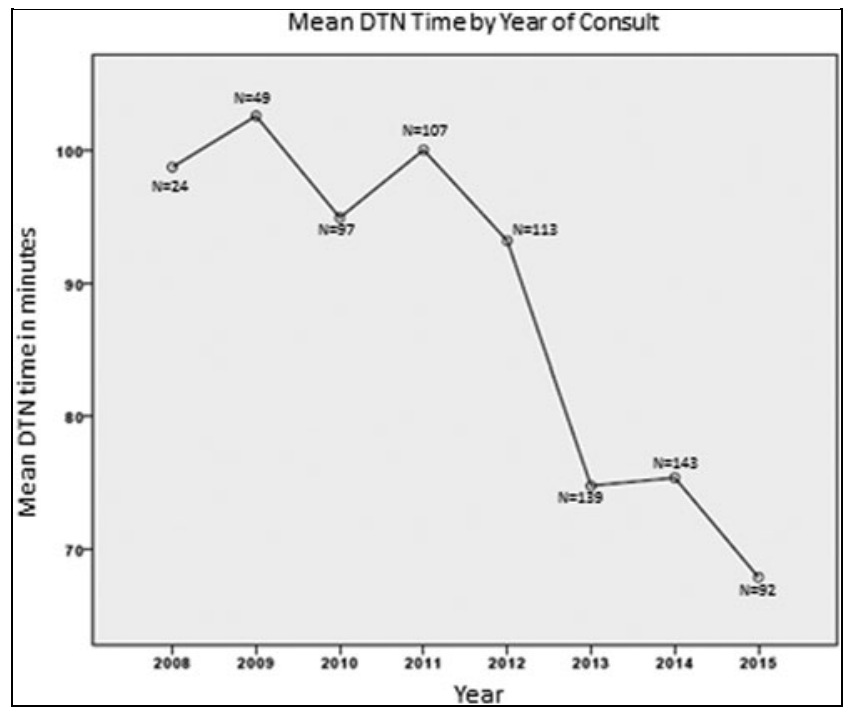




\section{ORAL PRESENTATIONS ABSTRACTS}

Results: Of the total 6116 consults performed over the time period, there was an average of 95.5 total consults/year among all 18 sites and an average of 8.3 consults/active site/year with documented arrival in ED and drug delivery times. The mean +/-SD DTN for all of the consults in all years was 85.4+/-42.0. As shown in the figure, we found a significant decrease in DTN over time at $(p<0.001)$. In the post-hoc test, the following pairs were determined to be significantly different (at p <0.05): 2008 vs. $2015 ; 2009$ vs. 2013, 2014, and 2015; 2010 vs. 2013, 2014, and 2015; 2011 vs. 2013, 2014, and 2015; 2012 vs. 2013, 2014, and 2015.

Conclusions: Among a statewide Telestroke network, the mean DTN time continued to decrease over the last 7 years, reflecting more efficient delivery of acute stroke treatment. Since more efficient stroke care delivery is associated with better outcomes, this may be a contributing factor to the overall improved stroke mortality within the state. During this time site visit frequency was increased providing more stroke education and specific case follow up. Since inception of the Telestroke program 7 sites have achieved Primary Stroke Center certification, a designation of The Joint Commission. These interventions and certifications played a role in improving DTN times and outcomes.

\section{Objectives}

1. Identify interventions to decrease door to needle times at telestroke sites

2. Describe the improtance of faster treatment of ischemic stroke patients

3. Apply various techniques to improve patient care and outcomes

\section{AMBULANCE MEASUREMENT OF NIH STROKE SCALE WITH} TELEMEDICINE: A FEASIBILITY PILOT STUDY

PRESENTER: Sarvam TerKonda, MD, Director Mayo Clinic Connected Care, Florida $^{1}$, William D. Freeman, MD ${ }^{1}$, Kevin M. Barrett, MD ${ }^{1}$,

Tarin Casadonte, $\mathrm{MS}^{1}$, Wendy Hattery, $\mathrm{MS}^{1}$, Lesia Mooney, $\mathrm{RN}^{1}$, Elizabeth Mauricio, $\mathrm{MD}^{1}$, Scott Silvers, $\mathrm{MD}^{1}$, Ranya Habash, $\mathrm{MD}^{2}$, David Miller, $\mathrm{MD}^{1}$, Carol Raper, RHIA ${ }^{1}$, Ben Brown, $\mathrm{MD}^{1}$, James F. Meschia, MD ${ }^{1}$, Rabih Tawk, $\mathrm{MD}^{1}$, Robert Wharen, $\mathrm{MD}^{1}$

${ }^{1}$ Mayo Clinic, Jacksonville, FL, ${ }^{2}$ EverBridge, Burlington, $M A$

Introduction: Ischemic stroke is a time-based disease, with better outcomes linked to shorter times from onset to treatment. Reducing door-to-needle times is associated with improved outcomes in TPA-eligible ischemic stroke patients. We hypothesized that remote ambulance-based measurement of the NIH stroke scale (NIHSS) using a HIPAA-compliant mobile platform immediately prior to arrival was feasible and would reduce door-to-needle times in patients eligible for treatment with IV rtPA.

Methods: We conducted a 2-part administrative, proof-of-concept feasibility pilot as part of our Comprehensive Stroke Center and stroke systems of care model (SSMC). The first part consisted of 'beta testing' a simulated stroke patient scenario in simulation along with EMS over aspects of the NIHSS measurement using a HIPAA-compliant video platform. The second part was a 60-day feasibility period in the mobile EMS environment with timed measurement of NIHSS on stroke patients en route to Mayo Clinic Hospital (Jacksonville, FL). All patients were treated by standard of care on arrival by the receiving neurologist/neurosurgery teams. Five EMS ambulances were equipped with a tablet device with LTE/4G-enabled wireless carrier with a secure HIPAA-compliant video platform to test proof of concept. The third part was a 30-day extension of the second part to improve logistical efficiency with EMS providers. The primary aim was video feasibility with timed measurement of the NIHSS. Secondary outcomes included satisfaction with the platform, comparing NIHSS measurement time against historical controls at our Stroke Center to calculate a theoretical 'DTN' time or impact. A P value $<0.05$ was considered significant by paired t test comparison of mean NIHSS measurement time against historical DTN.

Results: Part one of the pilot was successful in beta video testing a simulated patient scenario in 3 of 3 attempts. Part two was successful in video testing the mobile NIHSS measurement in 10 of $11(91 \%)$ attempts. The one video attempt that was unsuccessful was due to local LTE being turned off on the device, which was later fixed. Video signal was dropped twice during a severe thunderstorm, delaying, but not preventing NIHSS measurement in one case. NIHSS measurement time averaged $7.45 \mathrm{~min}$ in 10 successful cases (range 3 to $9.8 \mathrm{~min})$. Satisfaction was rated good to excellent from the neurologist perspective, and satisfied to very satisfied in 100\% of successful attempts with EMS, and poor with the one failed video attempt. Given the average NIHSS measurement time, a theoretical DTN was $38 \mathrm{~min}$ (7 to $45 \mathrm{~min}$ ) theoretical impact, $\mathrm{P}$ - value 0.9 via paired t test. A power calculation using these data and standard deviation in DTN of 40 min showed that this theoretical DTN would require a sample size of 197 patients to test this hypothesis with an alpha of 0.05 with $80 \%$ power.

Conclusion: This proof of concept pilot demonstrates that remote ambulance-based $\mathrm{NIH}$ stroke scale measurement is feasible albeit with technical limitations. The average time of the NIHSS measurement during transport was 7.4 minutes which could theoretically reduce door to needle times and allow patients to go direct to CT scan or other interventions but the study was underpowered to detect significant difference. A sample of approximately 197 patients would be needed to test this hypothesis adequately.

\section{REFERENCE}

1. Wu TC, Nguyen C, Ankrom C, Yang J, Persse D, Vahidy F, et al. Prehospital utility of rapid stroke evaluation using in-ambulance telemedicine: A pilot feasibility study. Stroke 2014; 45(8):2342-347.

\section{Objectives}

1. Define how telemedicine on an ambulance can help measure NIH stroke scale

2. Review measurement time of NIH in an ambulance

3. Discuss limitations of mobile telemedicine

MONDAY, MAY, 16, 2016

11:00 AM-12:00 PM

Monday, May, 16, 2016

\section{Session 26}

\section{Session Title: TELEICU MANAGEMENT IN RELATED SETTINGS}

MODERATOR: Herb Rogove, DO, FCCM, FACP, President and Chief Executive Officer

C30 Telemedicine, Ojai, CA

THE EFFECT OF TELE-ICU INNOVATION ON PROGRESSIVE CARE UNIT (PCU) PATIENT POPULATION

PRESENTER: Leslee Gross, Assistant Vice President of Telehealth, Louis Gidel, PhD, MD

Baptist Health South Florida, Coral Gables, FL

Introduction: The inclusion of an ICU telemedicine program as a major part of the critical care delivery paradigm has been implemented for $11 \%$ of critically ill patients in the U.S. as a perceived solution for the problem of access to adult critical care services.

Human Workforce: The increasing number and severity of critical care patients as the U.S. population ages, and the decreasing supply of critical care physicians available to manage the growing number of ICU patients supports a rationale for Tele-ICU. Higher acuity patients that were formerly cared for in ICUs are increasingly cared for in PCUs. Tele-ICU has the potential to address this critical care staffing shortage by enabling clinicians in one "command center" to remotely monitor, consult and care for ICU and PCU patients in multiple and distant locations. By increasing the number of ICU and PCU 
patients that critical care teams can manage through Tele-innovation effectively extends both the productivity and the reach of the specialists.

Finance: Policymakers have had a long-standing interest in knowing under what circumstances improved quality and safety also result in reduced healthcare costs. ICU quality initiatives in general and the Tele-approach in particular are ripe areas for expanding knowledge of the quality/cost tradeoff, not only because the ICU is a core clinical service in virtually all hospitals, but also because ICU and PCU care are large and growing contributors to rising healthcare spending.

\section{Questions About Return on Investment:}

1. Unknown impact on quality and costs.

2. There are a number of barriers, not only capital and operating costs, but also organizational and clinical staff resistance, technical incompatibilities, cross state licensure issues, and lack of payment for the Tele-ICU services.

3. Intensive care units are an essential and costly component in most U.S. hospitals; however, little is actually known about what staffing and work-process interventions produce the best balance of quality and

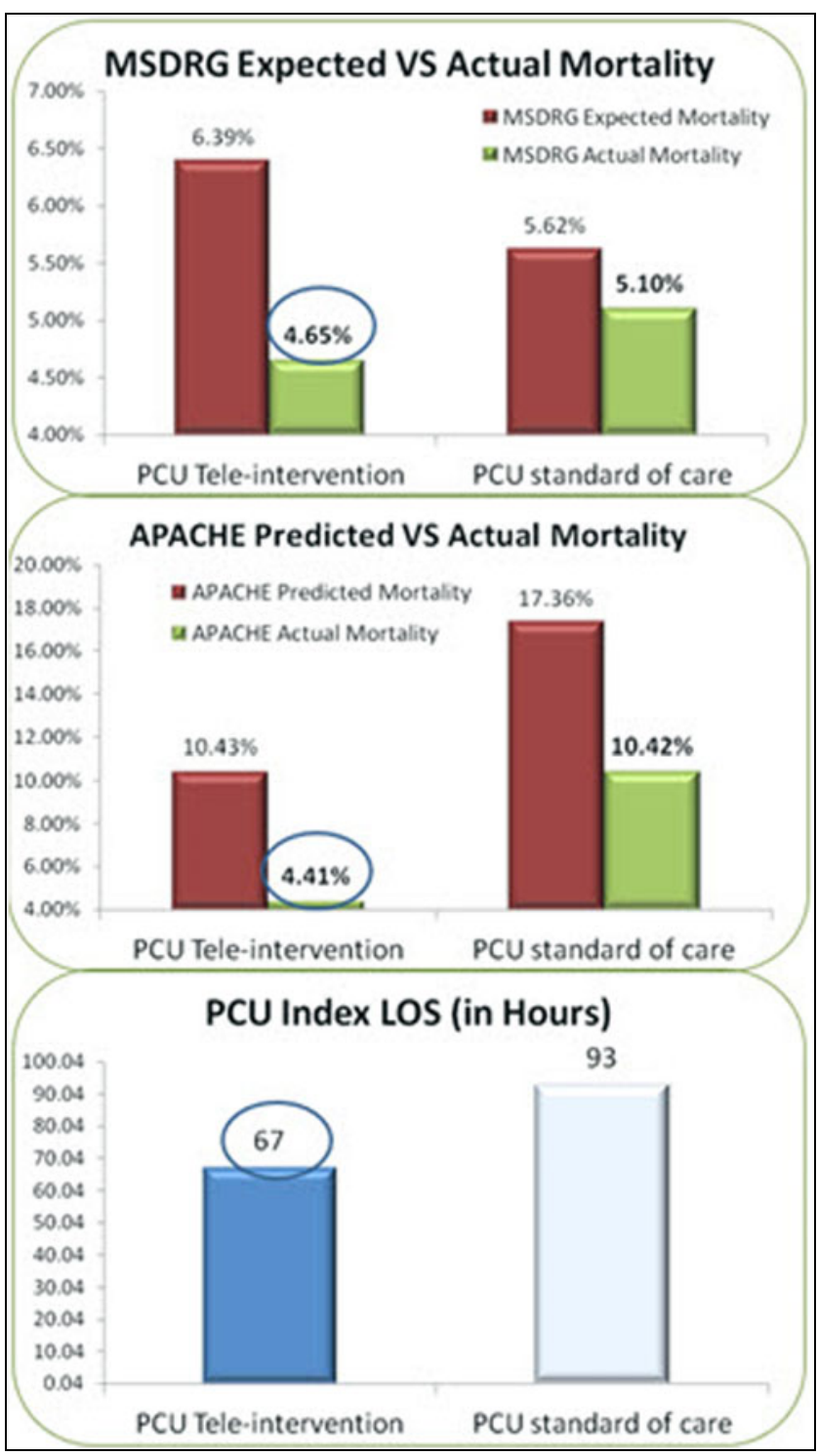

costs. Hospital clinical leaders hold strong views but have little objective information on which to judge the worthiness of Tele-innovation. 4. Substantial variation in the practice of ICU telemedicine exists, including qualifications of providers; ICU staffing models, leadership and governance; intensivist review for new patients; adherence to best practices; use of quality and safety information; and ICU physician sign out for their patients.

Methods: The optimal telemedicine technology configuration and dose tailored to ICU organization and case mix remain unclear. Tele-innovation's advanced monitoring, clinical decision-support functions and cognitive affordances have not been examined in PCU. Therefore, data about $n=13,421$ patients from 6 hospitals (Observational Case Control design) from Jan 2012 Mar 2015 were analyzed. PCU Tele-intervention $n=6,374$ and PCU standard of care control $n=7,047$. PCU inclusion time was defined as PCU Index $=$ first contiguous PCU census encounter $>24$ hours (time thresholds derived from greater than median LOS). Intervention group inclusion defined as $>24$ hours Tele-intervention during PCU Index time.

Results: We compared significant well established outcomes and quality measures between PCU Tele-intervention and PCU standard of care, namely, hospital length of stay (LOS), mortality, APACHE IV severity adjusted mortality and MSDRG severity adjusted mortality. The two groups were fairly balanced. Comparing outcomes in PCU Tele-intervention vs. PCU standard of care, respectfully, the intervention group is older (70+/-16 vs. $65+/-18, \mathrm{p}<0.001)$; of the patients who had MSDRG expected mortalities (6359, 7018), expected mortality $(6.39 \%$ vs. $5.62 \%, p=0.0025)$; however, actual mortality direction was reversed and lower (4.65\% vs. 5.10\%, $p=0.2444$ ). PCU Index LOS was shorter (67 hours vs. 93 hours, $p<0.001$ ). Of the patients who had an APACHE IV prediction (5852; 1319), predicted mortality $(10.43 \%$ vs. $17.36 \%, \mathrm{p}<0.000)$; however, actual mortality is lower $(4.41 \%$ vs. $10.42 \%$ vs. $\mathrm{p}<0.000)$.

Conclusions: In our population, Tele-ICU approach applied in PCU resulted in significantly decreased mortality and much shorter PCU Index LOS. These findings provide evidence of the effectiveness of Tele-innovation and validate the impact on quality and cost in the progressive care setting, providing a rationale for extension of access to Tele-PCU care services across broader hospital populations. Further investigation is needed to examine severity adjusted predictions across varying practice settings. Data analytics in healthcare is evolving into a promising field to attain insight from very large data sets and improve outcomes while reducing costs. Its potential is great; however there remain challenges to overcome. The next generation of research must provide clinicians, healthcare administrators, and policy makers with actionable data to guide truly effective implementation.

\section{REFERENCES}

1. Bashshur RL, Shannon GW, Smith BR, Alverson DC, Antoniotti N, Barsan WG, et al. The empirical foundations of telemedicine interventions for chronic disease management. Telemed J E Health 2014; 20(9):769-800.

2. Kahn JM. ICU telemedicine: from theory to practice. Crit Care Med 2014; 42(11):2457-8.

3. Kahn JM, Cicero BD, Wallace DJ, Iwashyna TJ. Adoption of ICU telemedicine in the United States. Crit Care Med 2014; 42(2):362-8.

4. Lilly CM, Zubrow MT, Kempner KM, Reynolds HN, Subramanian S, Eriksson EA, et al. Critical care telemedicine: evolution and state of the art. Crit Care Med 2014; 42(11):2429-36.

\section{Objectives}

1. Describe tele-innovation application in progressive care

2. Distinguish effect of tele-intervention on mortality and quality outcomes

3. Describe implications for practice and future research 


\section{CLINICAL AND FINANCIAL BENEFITS OF TELE-ICU MANAGEMENT} OF ICU BOARDERS IN THE EMERGENCY DEPARTMENT

PRESENTER: Michael Ries, MD, MBA, Medical Director Critical Care and eICU Cindy Welsh, RN, MBA, VP, Adult Critical

Care \& Medical Professional Affairs

Advocate Health Care, Oak Brook, IL

The practice of holding critically ill patients in the emergency department (ED) as boarders after they have been admitted to the hospital because no ICU beds are available can pose significant safety and quality risks. After a serious safety event of an ICU boarder, we integrated ICU telemedicine into the process of providing care for the boarded ICU patient. This new paradigm of care realized value-added benefit with continuous attention by critical care physicians and nurses, a 25\% downgrade of patients from ICU status which allowed admission to a lower acuity bed, reduced LOS in the ED, and increased delivery of evidence-based medicine. These successes led us to extend our tele-ICU - ED program to three additional hospitals in our healthcare system.

Prolonged boarding times result in an increase in morbidity and mortality in critically ill adult patients admitted to the ICU. One of our hospitals, which has one of the busiest ED's in the state, typically has $4-5$ and sometimes as many as 8 - 10 ICU boarders at a time. In 2014, ICU boarders spent 836,530 minutes being boarded in that one ED. ED boarding has been associated with other negative patient-oriented outcomes including increased medication errors, diversion of ambulances, and lower patient satisfaction. Boarding these patients can delay optimal care and tie up resources which render emergency room staff unable to provide best care to the other emergency room patients. This can be especially harmful to critical care patients who are sick enough to be in an ICU but instead receive inconsistent care in the emergency room which is not equipped technically and lacks proper specialized critical care trained physicians, nurses, and pharmacists. Traditionally, the only strategy to obviate this problem has been to optimize bed management to reduce boarding by improving the efficiency of hospital patient flow.

As our use of tele-ICU evolved over the last 12 years, multiple benefits have been noted: disease management by intervening early in acute situations; supplementary rounding to realize opportunities in disease management; population management of ICU patients; and, gap solutions in the process of delivering evidence-based medicine to our critically ill patients. After the adverse safety event, delays and omissions caring for ICU boarders were identified. The gap analysis of the process identified opportunities that could be resolved by our tele-ICU program, including a rethinking of standard practices.

The bedside intensivist, while physically not stationed in the ED, was able to function as the primary responsible physician because live video, data, vital signs, lab and radiology reports, etc., that streamed to the tele-ICU enabled that team to primarily manage the boarder patients. Tele-ICU supported the bedside ED nurses and physicians and, through focused management, the identification of early clinical changes. While changes in the care of shared ICU boarder patients occurred on many levels, the tele-ICU brought organizational clarity, improved clinical outcomes and financial benefit.

\section{REFERENCES}

1. Bernstein SL, Aronsky D, Duseja R, Epstein S, Handel D, Hwang U, Asplin BR. The Effect of Emergency Department Crowding on Clinically Oriented Outcomes. Acad Emerg Med 2009; 16(1):1-10.

2. Chalfin DB, Trzeciak S, Likourezos A, Baumann BM, Dellinger PR. Impact of delayed transfer of critically ill patients from the emergency department to the intensive care unit. Crit Care Med 2007; 35(6):1477-483.

3. Kulstad EB, Sikka R, Sweis RT, Kelley KM, Rzechula KH. Overcrowding is associated with an increased frequency of medication errors. Am J Emerg Med 2008; 28:304-09.

4. Engoren $\mathrm{M}$, et al. The effect of prompt physician visits on intensive care unit mortality and cost. Crit Care Med 2005; 33:727-32.

\section{Objectives}

1. Learn how telemedicine can bring organizatinal clarity to complex patient management

2. Demonstrate how gap analysis affords an opportunity for telemedicine to improve evidence-based practice adherence in the ICU patients boarding in the Emergency Room

3. The success of telemedicine will be determined less by what technologies you have and more by how you use them

\section{MONDAY, MAY 16, 2016}

1:15 PM-2:15 PM Monday, May 16, 2016

\section{Session 33 Session Title: FINANCIAL AND OUTCOME IMPACT OF TELEICU}

MODERATOR: Naomi Fried, PhD, Healthcare Innovation and Digital Health Leader

Boston, MA

\section{A COMBINED INTENSIVIST/TELEINTENSIVIST PROGRAM MODEL IMPROVES ICU OUTCOMES}

PRESENTER: P. W. Ludwig, MD, FCCP, President, V. Patel, D0, K. Ramos, LPN, BHSA, B. Ludwig, MBA, M. Marquez, FACHE NuView Health, Boca Raton, FL

Introduction: Our group has extensive experience with ICU telemedicine. Previous experience has demonstrated the need for a structured approach to ICU care, whether by daytime onsite Intensivist or via a Teleintensivist model. We recorded our results for a 1 year period after instituting a Community Hospital Intensivist program and in this study a combined Intensivist/Teleintensivist model of care.

Methods: The study hospital is a Community Hospital with a 19 bed ICU and approximately 1,000 ICU admissions per year. The baseline data is from 2013 during which time there was an Intensivist program onsite staffed by 2 daytime Intensivists with phone call coverage at night. We established a structured model for ICU care with a 12 hour daytime Intensivist and a 12 hour night Teleintensivist. There is a mandatory Intensivist consult for all ICU admissions during all hours in this combined Intensivist/Teleintensivist program. The telemedicine system utilized by the Teleintensivist allows simultaneous visualization of the patient and the on call Teleintensivist. The Teleintensivist also has full access to the EMR and PACs system. Both daytime and nighttime physicians followed a structured schedule including triage rounds, MDR rounds, formal handoff, evening Teleintensivist "walk rounds" and protocols for care. Data was collected for a 1 year period with a chart review on all ICU admissions. Data was compared to hospital obtained baseline data for the year prior to program start date.

Results: In the 12 month period after the program start there was a significant reduction in ICU mortality $18.9 \%$ to $10.53 \%(\mathrm{p}<0.01)$. The MPM predicted mortality for the period was $14.9 \%$. ICU LOS was reduced from 5.38 to 3.65 days. Total hospital LOS for ICU patients was reduced from 12.05 to 8.53 days. ICU readmission rate was less than 3\%. Other metrics including ventilator days/case, VAP, and CLABSI rates all averaged better than national benchmarks.

Conclusions: A structured Intensivist/Teleintensivist program instituted after a long term onsite traditional Intensivist model reported a significant improvement in patient care and outcomes. This combined model is both cost effective for the hospital and improves quality. 


\section{Objectives}

1. Combined Intensivist and Teleintensivist program

2. Structured schedule for Intensivist and Teleintensivist

3. Positive results from combined programs

EARLY ANALYSIS OF THE FINANCIAL IMPACT OF TELE CRITICAL CARE IMPLEMENTATION

PRESENTER: William Beninati, MD, Medical Director, TeleCritical Care, Andy Merrill, MStat, Marni Chandler, MSN

Intermountain Healthcare, Salt Lake City, UT

Introduction: Intermountain Healthcare established an adult telecritical care (TCC) program to engage a daily census of 150-170 patients distributed between 7 community hospital intensive care units (ICU) and 5 tertiary hospital ICUs. The objectives of this program are to improve clinical outcomes and reduce the cost of care.

Methods: The TCC program is based on data from Intermountain's electronic medical record supplemented by remote web viewing of bedside monitor data and audio-video conferencing with an internally developed platform. Intermountain's internally operated TCC support center is staffed continuously with critical care nurses and physicians. The nurses have a ratio of approximately 1 staff to 40 patients, perform proactive best-practice rounding on all patients, and provide reactive clinical support to the bedside. The physicians focus on prompt consultation and ongoing co-management for community hospital admissions, along with reactive support for all ICU patients. Implementation of nurse-only coverage for 20 beds began in May 2014 with a stepped addition of beds until full implementation in December 2014. Daytime physician coverage began in August 2014 and moved to continuous coverage in December 2014. The program did not take any steps specifically aimed at reducing costs. An initial financial analysis was conducted in May 2015. A change in net operating income was calculated based on the change in total variable costs for patients with an ICU admission compared with a pre-implementation. Change in payment related to reduced variable costs was also estimated. The ICU variable costs were adjusted based on patient age, acute physiology score, diagnosis group, month, and sex.

Results: The encounters in the analysis period normalize to 21,551 per year. The estimated annual decrease in payments received was $\$ 1,014,476$ in community hospitals and $\$ 3,354,364$ in tertiary hospitals. In community hospitals the observed cost/case was $\$ 4,397$ compared to an expected cost/ case of $\$ 4,697$ adjusting for patient age, acute physiology score, diagnosis group, month, and sex for an estimated annual savings of $\$ 1,014,476$. In tertiary hospitals the observed cost/case was $\$ 9,225$ compared to an expected cost/case of $\$ 9,410$ for an estimated annual savings of $\$ 3,354,364$. The impact on operating income was a decrease in costs of $\$ 4.36$ million and a decrease in payments of $\$ 3.34$ million for a favorable net operating income impact of $\$ 1.02$ million after one year of operation.

Conclusions: After 6 months of partial- and 6 months of completeimplementation of TCC we have seen a substantial net financial benefit. This does not yet offset the personnel and equipment costs for implementing TCC, but that was not expected at this point. Intermountain is moving to an accountable care model in which care is pre-paid. Under this model the benefit is much larger. A limitation of this study is the short implementation period and the lack of granularity for the specific areas of savings. This will be addressed in ongoing analysis.

\section{Objectives}

1. Describe a model for implementation of an internally-developed telecritical care program

2. Identify factors that can confound a pre- and post-implementation financial analysis

3. Discuss factors that contribute to the financial viability of a telecritical care program
MONDAY, MAY 16, 2016

4:15 PM-5:15 PM Monday, May 16, 2016

\section{Session 39 \\ Session Title: IMPROVING ACCESS TO CARE FOR BRAIN INJURY}

MODERATOR: Lawrence Wechsler, MD, Professor and Chairperson

Department of Neurology, University of Pittsburgh, Pittsburgh, PA

\section{DISRUPTING HEALTHCARE DELIVERY: USING TECHNOLOGY}

TO PLACE EXPERIENCED CLINICIANS ON THE FIELD

WITH INJURED PLAYERS

PRESENTER: Fadesola Adetosoye, MSc, Global Healthcare Solutions Leader -Telemedicine \& Integrated Care Management Dell, Inc, Silver Spring, MD

In an evolving healthcare ecosystem, patients, plans and providers must adopt innovative ways to improve care delivery using cost-appropriate methods. While telehealth services are becoming more technologically advanced and consumer focused, it becomes vitally important for health plans to adopt appropriate telehealth services that align with the needs of various population segment through optimal and innovative technological solutions. This session will focus on the latest technology and trends in telehealth to help you transform healthcare delivery and highlight the direction health plans and other organizations should consider in their interests in expanding their business offerings, ultimately driving more value-based care.

In partnership with VSee, Dell will offer a low cost, HIPAA-compliant, video telemedicine platform for Vsee software, which can connect remote physicians with patients anywhere in the world via the Internet. In this study, the system will connect a remote physician with an athlete on the field for the concussion consult. Dell will provide Windows 8 tablets with built-in highdefinition cameras, to facilitate the video consult.

At the end of the football season, UMMC's Center for Telehealth will provide metrics to the NFHS to show how often the system was used and to measure the effectiveness of on-field evaluation by a physician. The NFHS is interested in scaling and replicating this program broadly, so a thorough evaluation of the program will help the group determine if this technology can improve the way coaches and other athletic officials respond to concussive injuries. Results from the study are expected to be available within the first half of 2016

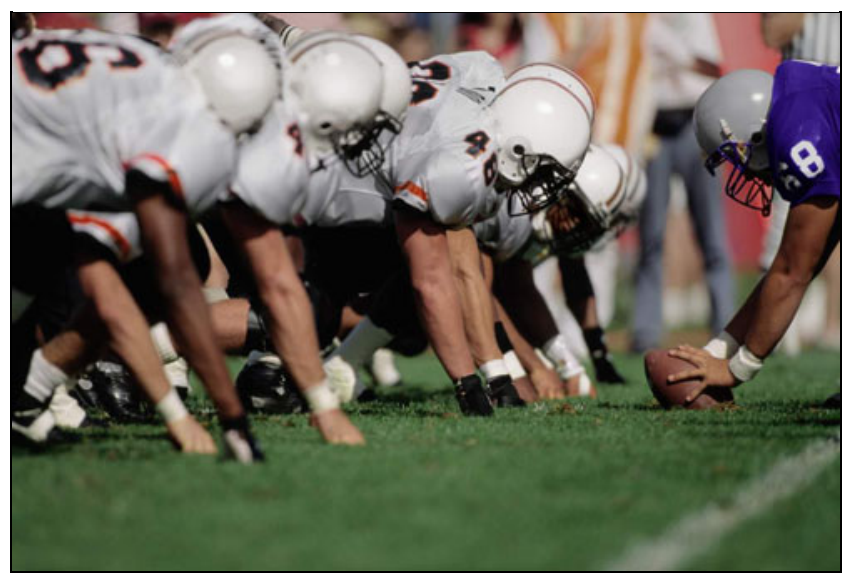




\section{ORAL PRESENTATIONS ABSTRACTS}

\section{Objectives}

1. Best practices for operating remote patient monitoring technologies

2. Identification of the most effective use cases for telemedicine and video conferencing

3. Impact of the new technology on patient and provider interactions within the healthcare ecosystem

\section{WOUNDED WARRIOR CARE MANAGEMENT PROGRAM USING MOBILE} TECHNOLOGY

PRESENTER: Nora Presson, PhD, Research Associate ${ }^{1}$,

Allison J. Borrasso, MS ${ }^{1}$, Steven Benso, $\mathrm{RN}^{1}$, Kathryn Edelman, MS ${ }^{1}$, John Dzivak, BA ${ }^{2}$, Naveen Khan, BA, PgDL ${ }^{2}$, Walter Schneider, $\mathrm{PhD}^{1}$, Ronald Poropatich, $\mathrm{MD}^{1}$, David 0. Okonkwo, MD, $\mathrm{PhD}^{1}$

${ }^{1}$ University of Pittsburgh, Pittsburgh, PA, ${ }^{2}$ PT Pal, Dallas, TX

Introduction: Traumatic brain injury (TBI) is a heterogeneous disorder with numerous clinical challenges for civilian and military populations. "TEAMTBI: Targeted Evaluation, Action and Monitoring" is a DoD-funded research program implementing multiple monitored clinical interventions for TBI patients. A key objective of this research is the development and initial testing of a patient protocol for integrating these multiple interventions. With advanced evaluation methods, a team of internationally-recognized experts identify and prioritize evidence-based therapies for patients who have sustained a TBI, assessing each patient across multiple trajectories, identifying relevant symptoms, and providing a sequenced, individualized treatment program to be monitored over a six-month period. Continuity of care between clinical and military coaches and the primarily veteran study population leverages existing mobile applications to remotely manage specific TBI trajectories that are impaired for each patient. The study was designed for monthly follow-up, allowing both online survey data collection and telephone conversation with clinical coaches; however, the complexity of both symptoms and treatments required more frequent communication from coaches, revealing a clear need for easy mobile access to study information (identified trajectories, treatment recommendations, contact information, access to surveys, documenting medication changes, etc.).

Methods: Participants travel to University of Pittsburgh and University of Pittsburgh Medical Center (UPMC) to undergo a 3.5-day comprehensive evaluation, completing assessments of sleep, mood, and vestibular, oculomotor, and neuropsychological function, as well as advanced neuroimaging studies. After testing, a multi-disciplinary case review identifies trajectories of concern, individualized goals, and treatment recommendations. Participants then depart Pittsburgh with a customized mobile TBI Toolkit, which includes: iPad with preloaded applications (e.g., Breathe2Relax for relaxation with diaphragmatic breathing; an object locator app), activity monitor, sleep kit, and object finders, along with targeted interventions (e.g., cognitive rehabilitation, specific sleep treatment, vestibular exercises, ocular-motor program). Clinical coaches provide telemedicine support and work with treatment providers for coordination of care. Clinician-to-patient communication occurs via telephone, e-mail, online surveys and/or text messaging and encompasses individualized prescribed treatment(s), outside referrals, goals, medication changes, and a review of monthly survey data to assess various TBI-related symptoms.

Results: As of September 2015, 27 patients (female $=6$; male $=21$ ) have been enrolled, with planned total enrollment of 120 . Of these, 7 have completed the goal-setting intake and initial progress monitoring process. All patients were assigned to one or more trajectories: the trajectories most often identified across subjects were Sleep $(n=19)$, Ocular-Motor/Vestibular $(n=20)$ and Mood $(n=21)$. Cognitive $(n=3)$ and other trajectories $(n=4)$ were also identified. Further evaluation and/or treatment recommendations were made for each identified trajectory. Of the 7 patients who completed goal-setting intake and progress monitoring, 5 met their goals, 1 made incremental progress, and 1 did not complete follow-up. In one example, the subject identified goals related to fatigue, vestibular/oculomotor, and cognitive TBI symptoms. This subject followed relevant treatment recommendations over the 6-month monitoring period, with coaching that adapted to an initial need for more frequent communication, and reported decreased fatigue, vestibular disturbances, and vision difficulties, which were associated with improvement in cognitive neuropsychological test scores in the absence of direct cognitive rehabilitation. Having identified patient and coach needs, our ongoing mobile development is adapting an established mobile app platform to allow coaches to administer specific exercises, monitor progress and app usage, take notes, and communicate with patients, as well as provide reminders and daily schedules for patients. This central app will include: notes, both clinician-only or patient-and-clinician; scheduling functions, with coaches/providers selecting exercises through a portal, patients receiving instructions to perform them via app, and therapists/coaches monitoring patient performance; and reminder functions to improve compliance. Key patient features include: $e x-$ ercise schedules and instructions, a repetition counting tool, and patient feedback. At monthly follow-up, coaches will be able to monitor patient performance on specific exercises, progress with outside referrals and recommendations, and survey results, as well as additional surveys 'ordered' for a patient by the coach, alerts/reminders for patients yet to complete, and a relational database of results. Upon completion, we will compare coaching with this central platform to coaching alone.

Conclusions: A comprehensive assessment is imperative to identify and prioritize primary areas of concern and effective treatment recommendations following TBI. Because multiple healthcare specializations are required for this comprehensive assessment and treatment approach, challenging, time-consuming clinical coordination of care is essential for patient compliance and accurate follow-through. A mobile platform will better support this multi-clinician, multi-treatment process, allowing a patient's "health story" to be shared by the patient and his/her treatment team. Sharing via mobile telemedicine encompasses both access and authorship. The application's primary purpose is to support care delivery, which in turn, will support better health. Preliminary data are encouraging for adoption and utilization of a mobile telemedicine platform to meet the complex needs of those recovering from TBI, and the program adoption of this platform will be a focus of our May presentation.

\section{REFERENCES}

1. Pavliscsak H, Little JR, Poropatich RK, McVeigh FL, Tong J, Tillman JS, et al. Assessment of patient engagement with a mobile application among service members in transition. J Am Med Inform Assoc 2016;23(1):110-18.

2. Poropatich RK, Pavliscsak HH, Tong JC, Little JR, McVeigh FL. (2014). mCare: Using Secure Mobile Technology to Support Soldier Reintegration and Rehabilitation. Telemed J E Health 2014; 20(6):563-69.

3. Chmura J, Presson N, Benso S, Puccio AM, Fissel K, Hachey R, et al. (2015). A High-Definition Fiber Tracking Report for Patients With Traumatic Brain Injury and Their Doctors. Mil Med 2015; 180(3S):122-34.

\section{Objectives}

1. Understand the complexities of TBI in a military population

2. Identify challenges of mobile health use in chronic care management

3. Apply mobile healthcare management tools to other clinical situations (e.g. total joint replacement)

\section{TUESDAY, MAY 17, 2016}

11:00 AM-12:00 PM Tuesday, May 17, 2016

\section{Session 47}




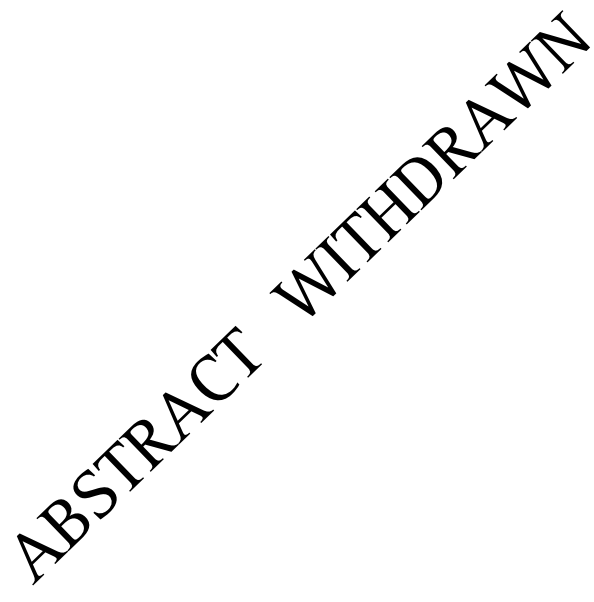

TUESDAY, MAY 17, 2016

1:15 PM-2:15 PM

Tuesday, May 17, 2016

\section{Session 55}

\section{Session Title: BUILDING AN EVIDENCE BASE FOR TELE-EMERGENCY CARE: REVIEWS OF THREE FEDERALLY FUNDED PROGRAMS}

MODERATOR: Boyd Gilman, PhD, Senior Researcher Mathematica Policy Research, Princeton, NJ

PRESENTER: Mandy Bell, MHA, eCARE Quality \& Innovation Officer Avera Health, Sioux Falls, SD
PRESENTER: James P. Marcin, MD, MPH, Professor

University of California, Davis, Sacramento, CA

PRESENTER: Nina J. Solenski, MD, Associate Professor University of Virginia, Charlottesville, VA

Background: In September 2014, the Office for the Advancement of Telehealth (OAT) awarded grants to six health systems through the EvidenceBased Tele-Emergency Network Grant Program (EB TNGP). The purpose of this program is to support implementation and evaluation of broad telehealth networks to deliver Emergency Department consultation services. The program primarily seeks to establish an evidence-base assessing the effectiveness of tele-emergency care. Each grantee is currently delivering 24-hour emergency consultation services defined as an immediate, synchronous, interactive audio/video connection between an ED specialist at the distant site and general practitioners at the originating site. OAT is also asking grantees to participate in collecting and reporting proposed tele-ED performance measures with a goal of each network individually, and collaboratively publishing analysis of the effectiveness of tele-ED services. In 2014, OAT has contracted with Mathematica to identify, pilot test, and recommend 25 tele-ED performance measures (by December 2015) that will contribute to the establishment of an evidence base for subsequent evaluation of the impact of tele-ED services on rural hospitals. The goal is to test the general hypothesis: Teleemergency services help to eliminate geographic disparities in care that may occur as a result of limited resources, manpower shortages, and long distances to specially trained providers.

Objectives: This panel will briefly describe 3 of the tele-ED program partners, the 25 performance measures, and the evaluation methods and measured outcomes of each program. Challenges to conducting rigorous evaluations of tele-emergency will be discussed along with potential solutions and lessons learned. Panel members will discuss the novel approach by OAT and Mathematica to develop common metrics that will allow for a multi-site analysis of the effectiveness of tele-emergency services.

Findings: Avera eEmergency provides general tele-emergency services to over 100 rural hospitals in the Upper Great Plains. Previous research has illustrated that these services support recruitment and retention of rural clinicians as well as support the economic viability of rural hospitals. As a part of the EB TNGP program, Avera is evaluating the effect of telemedicine on the timeliness of emergency care as well as the ER discharge disposition of the patient for 22 network hospitals. Additionally, Avera is measuring the impact of services on the staffing of rural emergency rooms. The University of California, Davis' Pediatric Telemedicine Program conducts an average of 2,800 inpatient and outpatient telemedicine consultations each year. UC Davis has previously demonstrated that a tele-emergency program specific for seriously ill children results in higher parent satisfaction, higher provider satisfaction, higher quality of care, safer care (lower medication errors), more appropriate care and more cost effective care. In this current project, there is an ongoing clustered, randomized, crossover trial of telemedicine versus telephone consultations among seriously ill children for several outcomes including quality of care, medication error rates and cost effectiveness of care. The University of Virginia, Stroke Telemedicine And Tele-education (STAT) Program provides acute stroke emergency consultation to surrounding rural hospitals. Through an academic research partnership with Specialist On Call Inc., STAT providers reach additional rural hospitals throughout the state, and have access to data from 47 rural hospitals nationally. An analysis of the combined programs demonstrates improved access, and eligibility for time sensitive treatments compared to no telemedicine, with trends toward meeting national guideline metrics set for larger hospitals. The effect of onsite and online stroke Teleeducation for ED staff on improving treatment metrics is ongoing, in addition to a cost analysis for the hub and spoke participants, including projected patient savings.

Conclusions: Developing a standardized approach to evaluation along with metrics that fit a wide variety of clinical programs including pediatric critical 
care, neurology, and general emergency medicine is challenging but feasible with concerted effort. It will require the ongoing cooperation of a multitude of experienced telemedicine networks to build an evidence base for tele-emergency care.

This project is supported by the Health Resources and Services Administration (HRSA) of the U.S. Department of Health and Human Services (HHS) under grant numbers G01RH27868, G01RH27872, G01RH27869, Evidence-Based Tele-emergency Network Grant Program for $\$ 800,000$, $\$ 799,327$ and $\$ 800,000$ respectively. This information or content and conclusions are those of the author and should not be construed as the official position or policy of, nor should any endorsements be inferred by HRSA, HHS or the U.S. Government.

\section{REFERENCES}

1. MacKinney AC, Ward MM, Ullrich F, Ayyagari P, Bell AL, Mueller KJ. The Business Case for Tele-emergency. Telemed J E Health 2015; 21(12):1005-11.

2. Potter AJ, Mueller KJ, MacKinney AC, Ward MM. Effect of tele-emergency services on recruitment and retention of US rural physicians. Rural Remote Health 2014; 14(3):2787.

3. Yang NH, Dharmar M, Yoo BK, Leigh JP, Kuppermann N, Romano PS, Nesbitt TS, Marcin JP. Economic Evaluation of Pediatric Telemedicine Consultations to Rural Emergency Departments. Med Decis Making ePub - May 7, 2015.

4. Yang NH, Dharmar M, Kuppermann N, Romano PS, Nesbitt TS, Hojman NM, Marcin JP. Appropriateness of disposition following telemedicine consultations in rural emergency departments. Pediatr Crit Care Med 2015; 16(3):e59-64.

\section{Objectives}

1. Describe the collaboration between three tele-emergency networks, describe the common performance measures being collected, and compare and contrast three unique approaches to tele-ED services across rural communities

2. Identify emerging best practices and approaches in evaluating the efficacy and impact of emergency telemedicine care

3. Summarize lessons learned from the Office for the Advancement of Telehealth's multi-site effort to develop a standardized approach to evaluation of tele-emergency care with a goal of building an evidence base for this telehealth model

\section{TUESDAY, MAY 17, 2016}

\section{3:00 PM-4:00 PM}

Tuesday, May 17, 2016

\section{Session 63 \\ Session Title: DEPLOYMENT OF PREHOSPITAL EMS TO EMERGENCY DEPARTMENT TELEMEDICINE FOR EMERGENCY AND ROUTINE TRANSPORTS}

MODERATOR: YiDing Yu, MD, Chief Innovation Engineer

Atrius Health, Newton, MA

PRESENTER: William Tollefsen, MD, Medical Director of EMS

South Shore Hospital, South Weymouth, MA

PRESENTER: Eugene Duffy, EMT-P, EMS Manager

South Shore Hospital, South Weymouth, MA

Emergency Medical Services (EMS) provide crucial prehospital alerts to Emergency Departments (ED) to enable hospitals to mobilize critical resources ahead of patient arrival. However, standard calls via radio or telephone provide limited patient information and point of care data, contributing to costly diagnostic and treatment delays.
Telemedicine between EMS and EDs offers a potential solution, but large-scale deployments of live-streaming solutions have been limited by poor connectivity and time burden on EMS and ED staff. This panel will discuss the planning, pilot, and deployment of a novel prehospital telemedicine platform for emergency 911 and routine transports in four communities in Eastern Massachusetts with a combined catchment population of 250,000.

South Shore Hospital, a Level 2 Trauma, Cardiac, and Stroke Center, partnered to implement a digital prehospital telemedicine technology with EMS agencies in four communities in Eastern Massachusetts. Each EMS agency had an exclusive 911 contract for the community and also provided routine transports to South Shore Hospital. South Shore Hospital is among the busiest EDs in Massachusetts, with approximately 100,000 ED visits per year. Wait times, ED throughput, and patient outcome metrics, including door-to-balloon, door-to-tPA, and patient satisfaction, are top priorities for South Shore Hospital.

A total of 12 ambulance trucks were outfitted with smartphones and HIPAA-compliant mobile software that allowed EMS to relay audio, photos, videos, EKGs, and clinical severity to the South Shore Hospital ED. All Emergency Medical Technicians (EMTs) and paramedics who may be deployed by 911 dispatch were trained on use the software and instructed to use the mobile application as the primary mode of prehospital communication. Data were received by ED physicians on a Web-based dashboard that provided centralized triage of all incoming ambulance data.

Over the course of a 1-month launch period, 121 patients transports employed prehospital telemedicine. System uptime was $99.9 \%$ at the end of 3 months. Priority 1, Priority 2, and Priority 3 cases accounted for $14.3 \%, 28.5 \%$, and $57.1 \%$ of all cases sent respectively $(p=0.017)$, averaged over all EMS agencies. Priority 1 cases included STEMI, trauma, stroke, and seizures. Discrete triage data were sent for 98.1\% of all notifications, which included vital signs, symptoms, and interventions. Supplemental verbal reports were provided for $58.1 \%$ of all cases, photos provided for $42.8 \%$ of cases, and EKGs sent for $20.9 \%$ of cases. Compared to Priority 3 cases, Priority 1 and 2 cases were more likely to include EKGs $(41.6 \%$ vs. $14.8 \%, p=0.038)$, but they were no more likely to include a photo or audio report.

In this case presentation, we will discuss how telemedicine can be successfully deployed for prehospital EMS communication. Partnerships with EMS agencies demonstrated that such a system can be used for both 911 emergencies and routine transports to the ED, with a high use of EKG transmission, photo documentation, and audio recording. In cases of heart attack, stroke, and major trauma, timely communication with the ED may accelerate diagnosis and treatment with life-saving interventions.

\section{Objectives}

1. Identify existing telemedicine solutions for EMS in the prehospital setting

2. Understand the process for piloting and deploying a prehospital telemedicine platform for EMS for 911 emergencies and routine transports

3. Demonstrate how user-centered design can improve workflow for EMS and EDs

\section{TUESDAY, MAY 17, 2016}

4:15 PM-5:15 PM

Tuesday, May 17, 2016

\section{Session 71 \\ Session Title: TELEMEDICINE TO SAVE NEWBORNS: TWO CASE STUDIES}

MODERATOR: John Chuo, MD, Attending Neonatologist and Medical Director of Telemedicine

The Children's Hospital of Philadelphia, Philadelphia, PA 


\section{ORAL PRESENTATIONS ABSTRACTS}

IMPACT OF TELEMEDICINE ON OUTCOMES AMONG CHILDREN TRANSFERRED FROM REFERRING EMERGENCY DEPARTMENTS TO A PEDIATRIC INTENSIVE CARE UNIT

PRESENTER: Parul Dayal, MS, PhD (Candidate), Nayla M. Hojman, BS, Jamie Kissee, MA, Jacqueline Evans, MD, PhD, Joanne E. Natale, MD, Yunru Huang, MS, Rebecca L. Litman, MA, James P. Marcin, MD, MPH

Department of Pediatrics, University of California Davis, Sacramento, CA

Introduction: Previous research has demonstrated that children transferred to Pediatric Intensive Care Units (PICUs) from referring Emergency Departments (EDs) have higher mortality rates than children admitted to PICUs directly from EDs at Children's Hospitals. Previous research has also demonstrated that the financial costs associated with the care of children transferred from referring EDs is higher due to heavier resource utilization, costs associated with emergency medical transport, and the burdens placed on families having to travel out of their local communities to receive care. Higher severity of illness and mortality rates among transferred children can be explained by the lack of necessary expertise, experience, equipment, and infrastructure in EDs outside of Children's Hospitals. Disparities in access to pediatric subspecialists could be partly addressed by the use of telemedicine. The use of telemedicine by pediatric critical care physicians to assist in the ED care of seriously ill children could facilitate the timely and appropriate stabilization of patients during the pre-transfer stage of the care process. The objective of this study was to compare outcomes among seriously ill children admitted to a Children's Hospital PICU from EDs with and EDs without access to pediatric critical care consultations using telemedicine.

Methods: We analyzed all pediatric patients ( $<18$ years old) admitted to the PICU at the University of California, Davis Children's Hospital directly from a referring ED between 2010 and 2014. We compared demographic factors (age and distance traveled) and clinical parameters (illness severity as measured by Pediatric Risk of Mortality III [PRISM III] score, PICU Length Of Stay [LOS], mortality and disposition) between patients transferred from EDs with and EDs without access to pediatric telemedicine. Two separate analyses were conducted: First, we compared factors among all children with and without access to pediatric telemedicine. Second, among hospitals that obtained access to pediatric telemedicine during the study period, we compared pre-telemedicine factors to post-telemedicine factors. We compared PRISM III scores between pre and post-telemedicine cohorts using a multivariable analysis adjusting for possible confounders such as age and distance traveled, as well as day and time of PICU admission. To evaluate the impact of telemedicine on mortality, we compared the $0 / E$ ratios (ratio of observed number of deaths to number of deaths predicted by the PRISM III score) of non-telemedicine, pre-telemedicine, and post-telemedicine cohorts.

Results: 582 patients were transferred to the PICU from 15 EDs having pediatric telemedicine capabilities and 524 patients were transferred from 60 EDs without access to pediatric telemedicine. During the study period, four EDs obtained telemedicine capabilities. Among this cohort, there were 95 patients in the pre-telemedicine cohort and 43 in the post-telemedicine cohort. On average, children transferred from EDs with telemedicine were younger (5.6 vs. 6.9 years, $\mathrm{p}<0.001)$ and traveled a greater distance $(72.4$ vs. 63.1 miles, $\mathrm{p}<0.05$ ) when compared to those transferred from EDs without telemedicine. In terms of clinical parameters, patients transferred from EDs with telemedicine were less sick (PRISM III score 3.2 vs. $4.0, \mathrm{p}<0.05$ ) when compared to patients transferred from EDs without telemedicine. Children from EDs with telemedicine also had shorter lengths of stay ( 3.1 vs. 3.8 days, $p=0.113$ ) and lower observed mortality ( $14 \%$ vs. $23 \%, p=0.067$ ) than children from EDs without telemedicine. After adjusting for age, distance traveled, and day and time of admission, children transferred from EDs with telemedicine had lower PRISM III scores than children transferred from EDs without telemedicine (Beta=
$-0.74, p=0.04)$. Among patients transferred from the four EDs that obtained telemedicine during the study period, we again found that the mean PRISM III scores were lower by 1.2 points $(p=0.03)$ in the post-telemedicine cohort compared to the pre-telemedicine cohort. The $0 / \mathrm{E}$ ratios of post-telemedicine, pre-telemedicine, and no-telemedicine cohorts were 0.88 (95\% CI 0.53-1.09), 1.07 (95\% CI 0.53-1.60) and 1.02 (95\% CI 0.71- 1.33).

Conclusion: Children transferred from EDs that use telemedicine to consult with pediatric critical care physicians arrive to the PICU less sick and have better clinical outcomes than those children transferred from EDs without telemedicine capabilities. This finding was not the result of simply transferring patients who were less ill. The implementation and use of telemedicine was associated with a decline in illness severity and improvements in the 0/E mortality ratios.

\section{REFERENCES}

1. Yang NH, Dharmar $M$, Yoo BK, Leigh JP, Kuppermann N, Romano PS, et al. Economic Evaluation of Pediatric Telemedicine Consultations to Rural Emergency Departments. Med Decis Making 2015; 35(6):773-83.

2. Webb $C L$, Waugh $C L$, Grigsby J, Busenbark D, Berdusis $K$, Sahn DJ, et al. Impact of Telemedicine on Hospital Transport, Length of Stay, and Medical Outcomes in Infants with Suspected Heart Disease: A Multicenter Study. J Am Soc Echocardiog 2013; 26(9):1090-98.

3. Dharmar M, Romano PS, Kuppermann N, Nesbitt TS, Cole SL, Andrada ER, et al. Impact of Critical Care Telemedicine Consultations on Children in Rural Emergency Departments. Crit Care Med 2013; 41(10):2388-95.2.

\section{Objectives}

1. Understand that there are disparities in characteristics impacting outcomes between children who are transferred to a children's hospital PICU from referring hospitals with and without telemedicine

2. Understand the role of telemedicine in improving clinical outcomes in critically ill pediatric patients

3. Evaluation of telemedicine as a possible intervention to improve availability of specialty care in a rural/remote hospital's settings lacking a PICU

\section{SAVING THE LIFE OF A NEWBORN, ONE VIDEO CONSULT AT A TIME}

PRESENTER: Jennifer Fang, MD, Fellow in Neonatal and Perinatal Medicine, Beth Kreofsky, BS, MBA, Joan Broers, MS, RN, Julie Jensen, MS, Christopher Colby, MD

Mayo Clinic, Rochester, MN

Introduction: The present-day system of perinatal healthcare delivery has improved the survival and long-term outcomes of many babies born in the United States. Unfortunately, those of certain racial backgrounds, socioeconomic groups and geographical locations share these gains inequitably. Our regionalized perinatal care system is primarily intended to ensure that high-risk deliveries occur in tertiary care centers. Myriad factors conspire to limit many expectant mothers' access to these advanced-level services, leading to delivery in resourcelimited hospitals (those in rural areas, small communities or poor inner-city neighborhoods). Providers at these facilities are ill equipped to respond to newborn emergencies and often provide a non-uniform level of care that deviates from established guidelines. Because high-risk resuscitations are routinely practiced at high-volume delivery centers such as ours, we will leverage our experience with telemedicine to disseminate best practices and help local providers conduct newborn resuscitations according to the highest standards of care, irrespective of resources. This program has the potential to significantly reduce the outcome disparities in our patient population improving access to neonatal intensive care. While these concepts and services are innovative, they also have 


\section{ORAL PRESENTATIONS ABSTRACTS}

their foundations in user-friendly and highly adaptable technology. Thus, this model may be generalizable to regions well beyond Minnesota.

Aims: In order to reduce the outcomes disparity among infants born to families with rural, resource-limited and minority ethnic backgrounds, we will implement a regional telemedicine service to diffuse best practices and afford the highest level of care to newborns requiring resuscitation in lower-acuity hospitals.

Findings: During the 30-month study period, the telemedicine service was utilized for 77 cases. It was activated by every health system site, with use proportional to delivery volume. All neonatologists had the opportunity to provide consultative services. More than half of all cases involved emergent newborn resuscitation guidance. A full third of the cases resulted in the newborn remaining in the hospital of birth thus avoiding costly medical transport and admission to the intensive care unit. We calculate a cost savings of over $\$ 600,000$ during these cases. The response to this service has been overwhelmingly positive with nearly $95 \%$ of providers indicating that they would use the service again and found it quite helpful. Initially, a wireless, tablet-based video-conferencing software was used. We recognized that that system resulted in poor first time reliability of connectivity. Additionally, there were issues with reliability of the wireless based system. We have spent extensive time and resources to develop a next generation system that is a cart based hard wired solution that may be brought to the patient's bedside. We have tested this system on multiple occasions and many sites and have determined that is offers significant advantages to our prototype. The next step in our project will be to disseminate a hard wired cart based telemedicine solution. We have been fortunate to collaborate with an institutional multidisciplinary technical team who can evaluate the $\mathrm{AV}$ inconsistencies and failures and determine root causes. Improvements to the system, policies, and infrastructure are ongoing.

Conclusion: Telemedicine is a cost-effective way to optimize patient outcomes and support local providers during neonatal resuscitations. We will continue to work to optimize our technical solutions and systems that are in place to improve and further disseminate this practice.

\section{REFERENCES}

1. Fang JL, Carey WA, Lang TR, Lohse CM, Colby CE. Real-time video communication improves provider performance in a simulated neonata resuscitation. Resuscitation 2014; 85(11):1518-22.

2. Colby $C E$, Fang JL, Carey WA Remote video neonatal consultation: a system to improve neonatal quality, safety and efficiency. Resuscitation 2014;85(2):e2930. 24269867.

\section{Objectives}

1. Describe current state processes for newborn resuscitation in the United States

2. Suggest an alternative system which may improve newborn outcomes and decrease medical malpractice

3. Explain the technology solutions that we have utilized

\section{Track: Chronic Care Room 200 CD}

\section{SUNDAY, MAY 15, 2016}

12:00 PM-1:00 PM Sunday, May 15, 2016

\section{Session 3} Session Title: BUILDING EVIDENCE FOR TELEHOSPICE

MODERATOR: Karla Washington, MSW, PhD, Assistant Professor University of Missouri, Columbia, MO
PRESENTER: George Demiris, PhD, Professor

University of Washington, Seattle, WA

PRESENTER: Debra Parker Oliver, MSW, PhD, Professor University of Missouri, Columbia, MO

Introduction: The Hospice Caregiving Research Network recently completed two randomized controlled trials to demonstrate the value of telehealth in the context of hospice and specifically to support informal caregivers of patients at the end of life. The two trials were ACTIVE (Assessing Caregivers for Team Intervention via Video Encounters) and PISCES (Problem Solving Intervention to Support Caregivers in End of Life Settings). These two trials are to our knowledge the largest randomized trials in hospice in general, and testing telehealth in hospice, more specifically. They were designed to address the many challenges that informal caregivers face when taking care of a loved one at the end of life. These challenges include lack of communication, lack of confidence in carrying out complex caregiving tasks, anticipatory grief, challenges of effective pain and symptom management, financial and other barriers, time management and physical fatigue. Our interventions targeted communication with the hospice professional team in order to improve pain management (ACTIVE) and overall problem solving ability during stressful times (PISCES).

Methods: For the ACTIVE study, we tested a telehospice intervention using a randomized controlled trial design with 446 caregivers from November 2010-April 2014. Hospice caregivers were randomized into two groups, usual care $(n=223)$ and intervention group $(n=223)$, who used web-conferencing or telephones to connect with the hospice care team during care plan meetings once every two weeks. We explored outcomes including the caregivers perceptions of pain medicine, caregiver quality of life, caregiver anxiety, and patient pain. Measures were taken at baseline and every two weeks for four weeks, monthly through 6 months, and every 45 days thereafter. Video recordings, field notes, caregiver and staff interviews assessed the intervention effects and facilitators and barriers to translation. In the PISCES study we also used a randomized control design to test the effectiveness of a 3-session problem solving therapy (PST) intervention on quality of life and anxiety delivered faceto-face (F2F) and via live-videoconferencing (VID) relative to an attention control (AC) comparison group. We recruited a total of 514 caregivers over 4 years [AC $(n=172)$, VID $(n=171)$, and F2F $(n=171)]$. Caregivers ranged in age from 19 to 100 (mean age 60.3 years), were predominantly female (75\%) and mostly adult children (55\%) or spouses/partners $(27 \%)$ of hospice patients.

Results: In the ACTIVE study we found that the intervention participants had a stronger belief that pain could be controlled and reported their patient's pain was better controlled than did the usual care participants 30 days after the patients death. Caregiver anxiety and patient pain were correlated and sub-analysis results indicated that caregivers of cancer patients benefit more than other hospice caregivers. For the PISCES study, compared to the attention control $(\mathrm{AC})$ group, caregivers in the $\mathrm{F} 2 \mathrm{~F}$ condition had postintervention reduced anxiety $(-1.31$ [95\% CI, -2.11 to 0.50$], \mathrm{p}=.002)$, improved emotional (.45 [95\% CI, .08 to .81], $\mathrm{p}=.02)$, social (.57 [95\% CI, . 19 to .95 ], $\mathrm{p}=.004$ ), financial (.57 [95\% CI, .21 to .93], $\mathrm{p}=.002$ ), and physical quality of life (.53 [95\% CI, .19 to .87], $\mathrm{p}=.002)$. Results were adjusted for referral agency, patient diagnosis, and bereaved status. There were no differences in caregivers in the videoconferencing (VID) condition compared to AC condition.

Conclusion: These two studies demonstrated the potential of telehealth in hospice as well as challenges and opportunities in conducting randomized clinical trials in telehospice. The ACTIVE study provided additional understanding related to cost, and facilitators and barriers to translation of a telehospice intervention. While technology made the intervention feasible, and the hospice philosophy is supportive of caregiver involvement, the intervention requires modification to make it practical. Specifically, 


\section{ORAL PRESENTATIONS ABSTRACTS}

caregiver education and emotional support need to be delivered outside the meeting. Use of additional technology platforms such as YouTube and Facebook may present opportunities to improve the intervention. Strengthening of the intervention and a new trial need to be conducted before the intervention is promoted within the hospice industry. Similarly, the PISCES study demonstrated the great potential of problem solving therapy for the hospice setting. Since face to face delivery was the most effective platform but may require increased resources, agencies may explore a hybrid delivery platform (combining in-person and video delivery) to capitalize the effects of the intervention and avoid challenges documented with a video-only delivery format.

\section{Objectives}

1. Demonstrate awareness of current evidence in telehospice research

2. Identify technical, ethical and practical challenges in conducting randomized controlled trials using telehealth-based interventions

3. Identify factors that lead to successful clinical trials in telehealth and more specifically in home-based telehealth interventions

\section{SUNDAY, MAY 15, 2016}

1:15 PM-2:15 PM Sunday, May 15, 2016

\section{Session 11 Session Title: CARDIAC MONITORING BEST PRACTICES}

MODERATOR: Kristi Henderson, DNP, NP-BC, FAEN, Vice President, Virtual Care \& Innovation

Seton Healthcare Family, Austin, TX

\section{REDUCTION OF HOSPITAL READMISSIONS THROUGH TELEHEALTH} AMONG HIGH-RISK CARDIAC PATIENTS

PRESENTER: David Taylor, RN, Supervisor of Telehealth Services Visiting Nurses Association (VNA) of the Rockford Area, Rockford, IL

In an effort to help Rockford Memorial Hospital lower its overall readmission rate, which hovered around 28\% in 2011, the Visiting Nurses Association of the Rockford area (VNA) launched a new initiative - the Heart \& Vascular Program - to provide remote patient monitoring services to high-risk congestive heart failure (CHF) patients who were not eligible for home care services under Medicare and insurance guidelines.

As part of an overall initiative to reduce hospital readmissions at Rockford Memorial Hospital, the Visiting Nurses Association of the Rockford Area introduced a new program they called the Heart \& Vascular Program, which identified high-risk congestive heart failure (CHF) patients who could benefit from remote patient monitoring (RPM) in both short- and long-term timeframes.

Despite the fact that the subset of cardiac patients treated for CHF were not typically eligible for home care services under Medicare guidelines, the staff at the VNA recognized that in most cases, RPM could prevent patients from being readmitted to the hospital.

To that end, the VNA worked with the Rockford Memorial Hospital cardiology group to create a new program that allowed any patient being treated by a hospital cardiologist to receive a remote patient monitoring device - whether they qualified for VNA home care services under Medicare or not.

In order to impact both the short-term hospital readmission reduction goals and the long-term health and well-being goals for patients, the VNA staff and hospital cardiologists created specific protocols around the RPM vital sign standards for patients in the program. Some vital sign parameters were consistent for all patients (such as weight), but others were customized (such as blood pressure) in order to ensure that clinicians got more accurate alerts and that all patients received the right level of attention.

The combination of standardized and customized vitals monitoring might have been difficult for the VNA to manage, given the breadth of its RPM services (with more than 85 patients monitored at any given point in time), but using Honeywell's software allowed the VNA nurses to easily import existing vital sign standards as well as set customized ranges in a patient record, making it simple to implement a customized threshold in their RPM system.

If the VNA telehealth nurses observed that a Heart \& Vascular Program patient was exhibiting out-of-normal range vital signs, they alerted the patient's cardiology staff, who then made contact with the patient and worked with them to address any issues. Through this early recognition of potential complications based on patient data points, they were able to provide early intervention such as: 1) a change to patient medication(s) without a visit to the doctor or 2) a visit to the physician's office without a trip to the emergency room.

While most cardiac patients in the Heart \& Vascular Program had monitoring devices for the standard 45-90 days, the VNA staff has the ability to be flexible with those timeframes. The ability to monitor patients in the longterm has allowed the VNA to impact and observe not only sustained lower hospital readmissions, but positive impacts to the long-term health and wellbeing of its patients.

\section{Outcomes:}

Prior to the implementation of the Heart \& Vascular program, the readmission rate for Rockford Memorial Hospital ranged between 25-28\%, on average. Following the launch of the program, overall readmission rates initially dropped to $17 \%$ after the program's first year, and to $14 \%$ after the second year.

And while the Heart \& Vascular program played a significant part in reducing the overall hospital readmission rates, they were also able to measure successful results within the readmission rates of their own program participants: rates for all-cause readmissions are now 7\%, with only 3.5\% of those related to CHF complications.

The patients have also reported increased levels of independence and an awareness that gives them the ability to self-manage their disease. The program has also provided patients' families with peace of mind, knowing their loved ones are being consistently monitored. In addition, the positive health improvements impact their greater communities and employers, as patients have been able to be more participative within those environments.

\section{Objectives}

1. Acquire a deeper knowledge around the utilization of telehealth to reduce readmission rates among high-risk patient populations - specifically cardiac groups

2. Assess whether the same type of telehealth program could be deployed within their own established organizational structure

3. Utilize the information provided to implement a telehealth program within their own organization

\section{VIRTUAL CARE PROGRAM FOR CONGESTIVE HEART FAILURE - \\ A CASE STUDY}

PRESENTER: Jim Lancaster, MD, Medical Director Cigna-HealthSpring, Nashville, TN

The Virtual Care Program for Congestive Heart Failure at Cigna-HealthSpring has been in operation since October of 2012. The program has been very successful across a number of measures and continues to grow and adapt to improve the results. The program has a number of objectives, including the following:

\section{- Promote self-management}

- Promote patient specific treatment 
- Provide patient specific intervention and education

- Improve quality of life

- Decrease and/or eliminate heart failure exacerbations and hospitalizations

Some of these outcomes can be measured objectively, but some require continual subjective monitoring by the clinical and operational staff involved in the program.

The program allows patients to receive care from the comfort of their own homes. It is a 90-day Virtual Care Program that gives the patient a dedicated nurse practitioner and a technology solution that includes a tablet computer, weight scale and blood pressure monitor. Monitoring includes daily biometrics, daily health questionnaires, and video conferences to discuss health concerns and review the biometrics directly with the patient. Additionally, the program includes a series of educational videos that enable the patient to make better health decisions and feel better about their health choices.

Proper planning and ongoing support for the program have been keys to the success. Additionally, the program has dedicated staff and senior-level sponsorship, which is important to any initiative such as this. The program has addressed several areas to improve results over time. This includes items such as improving internal communications with providers, tracking key operational metrics, and improving patient recruitment practices. Lastly, the program has an extremely dedicated, committed and enthusiastic clinical and operational staff that believes in the value of telehealth and remote patient monitoring.

This presentation will share some key learnings from a very successful remote patient monitoring program.

\section{Objectives}

1. Identify components of a successful remote patient monitoring program

2. Demonstrate how to change and adapt within a remote patient monitoring program to ensure success

3. Discuss actions for success and pitfalls to avoid in a remote patient monitoring program

\section{MONDAY, MAY 16, 2016}

\section{8:00 AM-8:45 AM Monday, May 16, 2016}

\section{Session 19}

\section{Session Title: TELE-COPD PROGRAM DEMONSTRATES REDUCED READMISSIONS AND COST SAVINGS}

MODERATOR: Karen Butterton, BS, Chief Strategy Officer

Barnes Healthcare Services, Valdosta, GA

PRESENTER: Amy Day, RRT, Director of Ventilation Services

Barnes Healthcare Services, Valdosta, GA

This session will highlight a retrospective study which evaluated COPD patients who were hospitalized two or more times within a year and transitioned to a COPD patient management program, which included monitoring those patients post discharge by TeleHealth. The results of the study showed that the readmission rate was reduced by $97 \%$ during the subsequent 12 months.

In the U.S., costs for hospital stays for patients with COPD as a principal diagnosis has been projected to be approximately $\$ 29.5$ billion. The readmission rate within 30 days of discharge for patients with COPD has been reported to be as high as $22.6 \%$. In this study, the proportion of COPD patients who were readmitted on two or more occasions decreased from 100\% (397 of 397 ) in the year prior to the initiation of the program to $2.2 \%$ (9 of 397) in the following year.

The session will demonstrate that better management of the COPD patient along with incorporating TeleHealth as one component of patient follow up and engagement allows health systems to not only significantly reduce readmissions and the associated high costs, but also improve the quality of life for some of its most complex, challenging, and chronic patients.

Retrospective Assessment of Home Ventilation to Reduce Rehospitalization in Chronic Obstructive Pulmonary Disease. Journal of Clinical Sleep Medicine, Steven Coughlin, Ph.D; Wei E. Liang, Ph.D, Sairam Parthasarathy, MD. Vol. 11. No 6, 2015.

\section{Objectives}

1. Gain a better understanding of a care continuum for COPD patients post discharge and how TeleHealth can be utilized to possibly prevent an acute exacerbation and subsequent readmission

2. Demonstrate the components required to successfully transition a COPD patient from the hospital to the home and ensure compliance with their therapies utilizing TeleHealth

3. Discuss the Barnes Healthcare COPD program specific to the study and published outcomes demonstrating a reduction in hospital readmissions

MONDAY, MAY 16, 2016

11:00 AM-12:00 PM

Monday, May 16, 2016

\section{Session 27 \\ Session Title: ADDRESSING THE NEEDS OF PATIENTS WITH MULTIPLE CHRONIC CONDITIONS THROUGH A COMPREHENSIVE TELEHEALTH PROGRAM}

MODERATOR: Todd Evans, BS, Director, Health Industries $P w C$ Healthcare, Chicago, IL

PRESENTER: Brian Rosenfeld, MD, Vice President and Telehealth Chief Medical Officer

Philips Healthcare, Andover, MA

PRESENTER: Deb Dahl, MBA, BSE, Vice President Patient Innovation Banner Health Network, Phoenix, AZ

Changes to and enhancements of telehealth technologies are fundamentally changing the way care can be delivered to certain populations. These changes facilitate new capabilities to address population health management in ways that have not been possible in the past, creating opportunities for collaboration among providers to anticipate patient needs before they escalate to threshold and more expensive levels.

Banner Health Network recently announced that it had delivered its best-ever results in the third successful year as part of the Medicare Pioneer Accountable Care Organization (ACO). Banner attributes its success to a number of innovative programs, including the Banner iCare program which targeted "super-users" patients with multiple chronic conditions who comprise approximately 5\% of patients yet utilize an estimated 50\% of healthcare resources. For this group of medically complex patients, healthcare needs - and remote management needs are very different. In 2013, Banner partnered with Philips Healthcare to pilot a telehealth-enabled program that uses "high-tech" technology and "high-touch" services to address the needs of this unique population. The "high-tech" component of the program includes a variety of telehealth technologies including remote monitoring and a specially designed Personal Health Tablet (PHT) to engage and communicate with the remote care team through two-way audiovisual software and messaging. Additionally, the PHT also delivers videos and surveys in the home. The "high-touch" component includes assignment of a personal Health Coach to help each patient measure his/her health and to deal with their psycho-social needs. Other members of the team include a team "quarterback" who keeps work assignments flowing, and a specially trained clinical team that includes an intensivist primary care physician who knows how to and likes to deal with this unique patient population. 


\section{ORAL PRESENTATIONS ABSTRACTS}

The data from the initial pilot program of 132 patients was very promising. Banner saw a $27 \%$ reduction in cost of care, a $32 \%$ reduction in acute and long term care costs, and a 45\% reduction in hospitalizations. The program was so successful that Banner increased the number of patients in the program to 500, $90 \%$ of whom are Medicare patients.

There were a number of learnings from the pilot program, including the challenges around identifying the level of patient support required to ensure the program's success. Banner found that patient claims data history alone was not sufficient and worked with behavioral psychologists to design a patient survey called the "MAY" or "More About You," scoring mechanisms to determine the patient's level of depression, and identification of the patient's behavioral phenotype to ensure that patient messaging and support is tailored to the individual's behavioral needs. The panel also will discuss the make-up of the remote care team, including the necessary qualifications and characteristics needed to make a program targeted to this complex population successful.

Participants will also learn about assumptions that were made in the beginning of the pilot which proved to be incorrect and how the program was adjusted to better meet the needs of the individual patients and the need to deliver high quality, cost-effective care.

Both Congress and the Medicare program have made this population a priority. This program demonstrates that enabling telehealth technologies when paired with the appropriate services can address the needs of patients with multiple chronic conditions and is the only cost effective and feasible way to meet the needs of a growing population.

\section{Objectives}

1. Recognize the challenges of dealing with patients with multiple chronic conditions that represent a relatively small population $(<5 \%)$ but a disproportionate cost (50\%)

2. Plan how best to integrate enabling telehealth technologies into programs designed to meet the challenges of this unique patient population

3. Understand the necessary clinical and behavioral health components necessary to achieve desired clinical and economic outcomes

MONDAY, MAY 16, 2016

4:15 PM-5:15 PM Monday, May 16, 2016

\section{Session 40}

\section{Session Title: TELEHEALTH \& REMOTE PATIENT MONITORING - FEDERAL OPPORTUNITIES AND CHALLENGES IN 2016 AND BEYOND}

MODERATOR: Brian Scarpelli, JD, Director, Government Affairs Telecommunications Industry Association, Arlington, VA

PRESENTER: Robert Jarrin, JD, Senior Director, Government Affairs Qualcomm, Inc, Washington, DC

PRESENTER: Crystal Riley, PharmD, MHA, MBA, Manager, Healthcare Policy

Baxter Healthcare Corporation, Washington, DC

Clinical evidence has demonstrated that the use of telehealth and remote monitoring of patient-generated health data improves care, reduces hospitalizations, helps avoid complications and improves satisfaction, particularly for the most chronically ill, and those in remote geographies. Yet the U.S. Congress and the Center for Medicare and Medicaid Services (CMS) continues to struggle to adopt meaningful policies that will ensure these solutions are fully leveraged. Join this panel to hear from key stakeholders about current and emerging telehealth and remote monitoring opportunities in Federal policy in the 2016 timeframe and beyond.

\section{REFERENCES}

A non-exclusive list of supportive references and works that are cited in this presentation are:

1. U.S. Agency for Healthcare Research and Quality (AHRO) Service Delivery Innovation Profile, Care Coordinators Remotely Monitor Chronically III Veterans via Messaging Device, Leading to Lower Inpatient Utilization and Costs (last updated Feb. 6, 2013), available at http://www.innovations.ahrq.gov/ content.aspx?id=3006

2. Hindricks, et al. Implant-based multiparameter telemonitoring of patients with heart failure (IN-TIME): a randomised controlled trial. Lancet 2014; 384(9943):583-90.

3. Darkins, Telehealth Services in the United States Department of Veterans Affairs (VA), available at http://c.ymcdn.com/sites/www.hisa.org.au/resource/resmgr/ telehealth2014/Adam-Darkins.pdf

4. Darkins A, Ryan P, Kobb R, Foster L, Edmonson E, Wakefield B, Lancaster AE. Care Coordination/Home Telehealth: the systematic implementation of health informatics, home telehealth, and disease management to support the care of veteran patients with chronic conditions. Telemed J E Health 2008;14(10):1118-26.

5. Mehrotra A, Paone S, Martich GD, Albert SM, Shevchik GJ, A comparison of care at e-visits and physician office visits for sinusitis and urinary tract infection. JAMA Intern Med. 2013;173(1):72-74.

6. Clark R, Inglis S, McAlister F, Cleland J, Stewart S. Telemonitoring or structured telephone support programmes for patients with chronic heart failure: systematic review and meta-analysis. BMJ 2007; 534(7600):942.

7. Quinn C, Shardelll M, Terrin M, Barr E, Ballew S, Gruber-Baldin A. ClusterRandomized Trial of a Mobile Phone Personalized Behavioral Intervention for Blood Glucose Control. Diabetes Care 2011; 34(9):1934-42.

\section{Objectives}

1. Demonstrate awareness of federal legislative and regulatory developments and opportunities specific to telehealth and remote patient monitoring

2. Identify key Federal legislative and regulatory barriers to realizing the potential of telehealth and remote patient monitoring

3. Determine priority venues for the most effective advocacy of views on telehealth and remote patient monitoring in the 2016 timeframe and beyond

\section{TUESDAY, MAY 17, 2016}

11:00 AM-12:00 PM Tuesday, May 17, 2016

\section{Session 48 Session Title: BENEFITS OF REMOTE PATIENT MONITORING}

MODERATOR: Lisa Roberts, PhD, Senior Vice President, Government AMC Health, New York, NY

INTEGRATION OF SPECIALTY ACUTE TELE-CARE WITH CHRONIC DISEASE MANAGEMENT

PRESENTER: Eric Dandes, MHA, Director of Operations and Analytics, Kaelin DeMuth, MS, Michael Shen, MS, MD, FACC

Duxlink Health, Sunrise, FL

Introduction: One of the key challenges in controlling healthcare expenses is managing patients with chronic diseases in order to prevent acute episodes that lead to unnecessary emergency room visits and hospital admissions or readmissions. Therefore, population-based chronic 


\section{ORAL PRESENTATIONS ABSTRACTS}

disease management integrated with effective acute episodic care that is managed by specialty may be a necessary approach in dealing with the paradoxical growing burden of chronic diseases with a shortage of primary care physicians in the U.S. How to integrate acute episodic care with routine chronic disease management under specialty physicians using specialized disease-driven clinical guidance has yet to be established. The project objective is to study and develop a specialty guided acute care network based on chronic disease management (CDM) in patients with cardiovascular diseases (CVDs).

Methods: Remote patient monitoring (RPM) devices (blood pressure, glucometer, weight scale, oximeter, and 12-lead electrocardiogram (ECG)) were provided to patients with CVD risk factors. High-risk patients with angina/coronary artery disease (CAD) and congestive heart failure (CHF) were included in the Specialty Network for Acute Care (SNAC) services for $24 \times 7$ monitoring and remote clinical care. Monitored data at the baseline was compared with those through telemedicine. Clinical outcomes, including acute myocardial infarction (AMI) and CHF readmissions, as well as Framingham risk scores (FRSs) were tracked and compared to clinical guidelines over a 12-month period.

Results: The SNAC system was developed in South Florida for approximately 100 patients with at least two or more chronic diseases in the cardiovascular specialty. As a result of the SNAC system, there was a 14\% drop in systolic blood pressure, and a 12\% drop in diastolic blood pressure, which resulted in a 13\% drop in the FRS. The SNAC system has now expanded to over 450 patients/month as a part of the Centers for Medicare and Medicaid Services (CMS) Innovation Program under Catholic Health System, with 8 hospitals in South Florida for transitional care, home care and the CDM-based SNAC program.

Conclusions: The preliminary data from the SNAC program has demonstrated a success in controlling chronic diseases compared to national clinical guidelines, with $10 \%$ of patients successfully treated for AMI and CHF. Integration of acute episodic care with routine chronic disease management under the specialty physician guidance is a new model to control readmissions and reduce healthcare costs.

\section{Objectives}

1. Analysis of the benefits of remote patient monitoring in congestive heart failure patients

2. Application of chronic disease management SNAC model

3. Analysis of healthcare savings using remote patient monitoring

\section{TESTING THE VALUE OF REMOTE PATIENT MONITORING - TELEHOMECARE - IN DIABETES, MENTAL HEALTH AND CHRONIC KIDNEY DISEASE}

PRESENTER: Laurie A. Poole, BScN, MHSA, Vice President, Telemedicine Solutions

Ontario Telemedicine Network, Toronto, ON, Canada

Telehomecare, an initiative of the Ontario Telemedicine Network (OTN), has delivered remote home monitoring and health coaching to more than 7,000 patients with chronic obstructive pulmonary disease (COPD) and congestive heart failure (CHF). The initial phase of the program proved the effectiveness of this model of care, with data reflecting patient satisfaction and reductions in healthcare resources. An average 50\% reduction in hospital admissions and visits to the ER have been reported by program delivery sites, both during the program and six months post discharge. Building on the success of the program, and in addition to rolling out Telehomecare across the province of Ontario, efforts are now underway to test the value of this innovative remote patient monitoring (RPM) approach for new patient groups to determine the efficacy and feasibility of new or streamlined models of care. Following the development of a high-level research framework, demonstration projects have launched targeting three specific patient populations. OTN's work with key stakeholders and community partners led to the identification of three priority chronic diseases - other than COPD and/or CHF - for which management in the community has been determined as a need: chronic kidney disease (CKD), diabetes and mental health.

These proposed demonstration projects also align closely with specific provincial government objectives within these priority areas. OTN is partnering with stakeholders, including a hospital and renal network, to establish a Telehomecare solution to support home peritoneal dialysis patients. OTN is also expanding Telehomecare to a pilot group(s) of diabetes patients, enabling a new mobile health (m-Health) model of care for children, youth and adults in up to three health authorities in the province. Finally, partnering with leaders in mental health in Ontario, OTN is establishing an alternate care model that provides digital mental health services including peer support forums and other self-management tools, all guided by mental health professionals. These patient groups have been selected according to the potential for RPM to have a significant impact on the healthcare and cost issues associated with these conditions.

Studies have shown that the $10 \%$ of patients with multiple chronic conditions account for up to $79 \%$ of healthcare costs and these costs will continue to increase as the population ages. Providing access to care in the home and the community for diabetes, mental health and CKD can both enhance the management of chronic illness and patient well-being and significantly bend the cost curve for these conditions. Remote monitoring differs from more consumer-oriented solutions for the "well" population in that it requires the involvement of healthcare providers and a timely and clinically appropriate response to data or health information submitted by patients. Innovative m-Health applications are being used for these projects to facilitate patient data collection and access to healthcare providers for patients who need support but may have difficulty accessing care and adhering to treatment. This presentation will focus on lessons learned, available outcomes data and how these interventions are optimizing self-management and quality of life for patients.

\section{Objectives}

1. Understand the potential for remote home monitoring and m-Health applications to support various patient populations

2. Describe the influence of $m$-Health initiatives on access to care and healthcare utilization

3. Evaluate the impact of $m$-Health initiatives on patient self-management and quality of life

\section{ECONOMICAL IMPACT OF A TELEMONITORING PROGRAM FOR HIGH} COMPLEXITY NON COMMUNICABLE DISEASES PATIENTS: A COMPARATIVE STUDY

PRESENTER: Xavier Urtubey, MD, MBA, Chief Executive Officer, David Decker, MA/MS, MPH

${ }^{1}$ AccuHealth, Santiago - Las Condes, Chile, ${ }^{2}$ CBS, Santiago - Las Condes, Chile

Introduction: AccuHealth is the first mover in the Telehealth Chilean industry, offering High-Value Evidence-Based Telemonitoring services for the private and public health system through a win-win disruptive patientcentered business model. Its core business aims mostly at out-of-control Chronic Disease patients management, creating a virtuous cycle by equilibrating them from a metabolic and clinical perspective, lowering the related expenses.

Methods: AccuHealth partners since 2014, initially in Chile, with a major privately owned third-party-payer to create and implement a Chronic Disease Patients Telemonitoring Program for High Complexity Non Communicable Diseases (NCD) beneficiaries including one or a combination of the following 4 diseases: Diabetes Mellitus (DM 1 \& 2), High Blood Pressure, Congestive Heart Failure (CHF) and Chronic obstructive pulmonary disease (COPD). The tele- 
monitoring program follows a telemonitoring plan of a series of daily protocols pre-established with the patients GP team including 1) biometric measurements (such as glucose, oxygen saturation, Blood Pressure, etc.), 2) an anamnesis intelligent threshold questionnaire, 3) the analysis of the patients metabolic and clinical patterns disruptions and 4) a behavioral change, all of them interpreted by a specialized team of Health Professionals supported by a data-mining predictive platform. Due to an accurate knowledge of potential Return On Investment, AccuHealth proposed to the HMO an alternative to the Fee-for-Service payment model with a pricing based on Saving Sharing. To be able to perform such an alternative, AccuHealth had to develop a methodology of Chronic Disease patients' identification and classification, yearly costs calculation and economical impact that would satisfy both the Telemonitoring team as well as the Health Insurance Company.

Results: In July 2014, the HMO and AccuHealth started proposing to its more complex NCD beneficiaries that they participate with no expenses on their side of the program, and in August the first patients started being Telemonitored. In that same month, 55 patients joined the program, followed by 87 in September, 130 in October and so on to reach a total of 831 patients recruited by end of April 2015. By the end of June 2015, 143 patients had dropped the program and 688 remained. By July 2015, the HMO made an analysis regarding outcomes of the first 10 months of the program. Regarding health impact, $60 \%$ of telemonitored patients were stabilized, 7\% increased their complexity level and 33\% decreased the NCD associated level of complexity. During the same period, the HMO conducted a retrospective economical impact study between the 688 patients that were on the program for at least 3 months or more and a control cohort with the same volume and characteristic of NCD's beneficiaries that never entered any Chronic Diseases Telemonitoring Program. From August 2014 until end of June 2015, the telemonitored cohorts decreased their NCDs-related cost around $1,250,000$ USD as during the same period the control cohorts increased their NCDs-related cost up to 225,000 USD, creating a savings gap of almost 1.5 Million USD for the period for an increasing number of patients.

Conclusions: Though further economical impact's analysis are still required, this study presents a clear economical impact of NCDs on telemonitoring vs. non-telemonitored patients.

\section{REFERENCES}

1. Trommer G. Digital health: bridging the gap between promise and reality. Biomed Instrum Technol 2015;49(3):182-87.

2. Asch DA. The Hidden Economics of Telemedicine. Ann Intern Med 2015; 163(10):801-02.

\section{Objectives}

1. Acquire an understanding of potential methodology on impact evaluation

2. Sharing commercial model international best practices

3. Achieve a better understanding of the review process for chronic disease cost evaluation

\section{TUESDAY, MAY 17, 2016}

1:15 PM-2:15 PM

Tuesday, May 17, 2016

\section{Session 56 \\ Session Title: MEASURING THE ABILITY \\ OF PREDICTIVE ANALYTICS AND HOME MONITORING TO REDUCE AVOIDABLE HOSPITALIZATION FOR COPD}

MODERATOR: Jack Kreindler, MD, Founder, Chairman, Chief Medical Officer

Sentrian, Inc., Aliso Viejo, CA
PRESENTER: Steven Steinhubl, MD, Director, Digital Medicine Scripps Translational Science Institute, La Jolla, CA

PRESENTER: David Ramirez, MD, Chief Medical Officer Caremore Health Plan, Cerritos, CA

Introduction: Chronically ill patients, such as those with congestive heart failure (CHF), chronic obstructive pulmonary disease (COPD) or diabetes, experience frequent hospitalizations and other expensive acute care interventions. Many of the hospital admissions occur after long intervals when there is no contact between patients and their clinicians. To close that communication gap, the idea of monitoring such patients at home has been around for at least 20 years. However, early generations of remote patient monitoring made several oversimplified assumptions. They focused on patients with one disease, such as CHF, and ignored the patients' comorbidities. The systems further assumed that the state of the one disease could be represented by a single measurement, such as patient weight for CHF. Those assumptions served to simplify the monitoring effort but resulted in many false positives. Lastly, first generation monitoring systems did not evaluate patients based on the likelihood that monitoring would be beneficial. At most, they chose patients at high risk for admission, but not for the possibility of altering outcomes.

Methods: In contrast with the current approach, the panel will describe the results of an interventional study of the value of home monitoring of physiologic parameters in individuals diagnosed with COPD by a Medicare managed care organization. The study will evaluate the utility of a rules-based analytic system, using home monitoring data combined with other longitudinal healthcare data to reduce avoidable acute care events (such as hospitalization or emergency department (ED) visits) by predicting deterioration in patients with COPD.The study will be carried out within the structure of the CareMore Medicare Managed Care Program. CareMore, an Anthem subsidiary, provides ongoing care for patients with chronic disease in California, Nevada, Arizona and Virginia. The study focus on patients managed at CareMore centers in California, with patients from other states included as necessary to achieve the target numbers. The panel will compare outcomes at one year between 1,000 active treatment individuals with COPD (and many with additional conditions like CHF and diabetes) and 1,000 matched controls receiving ongoing standard of care. Subjects are identified based on a robust set of inclusive criteria that includes a broad age range. In addition to the usual medical care, patients are provided with home monitoring devices that wirelessly record multiple physiologic data on a regular schedule. The data is analyzed by an event-processing engine that continuously evaluates newly acquired measurements against a pre-defined set of rules. A set of rules is termed a 'disease model'. The rules established are based on the devices assigned to the patient. Thus, patients with COPD and CHF might have rules based on the use of devices for peak flow, weight, oxygen saturation, pulse and blood pressure, constituting a disease model. The system allows an unlimited set of rules to be specified for a given model, which in turn enables complex patterns to be specified and detected.

Results: Preliminary results support the hypothesis that, by identifying patients more likely to benefit from home monitoring, choosing appropriate devices, evaluating day-to-day changes and longer term trends in measurements of multiple parameters and detecting patterns that predict deterioration days in advance of the patient becoming severely symptomatic, modest interventions can reduce avoidable hospitalization.

Conclusions: If the results continue to support the hypothesis, then the findings could significantly reform disease management not only for COPD, but for other chronic diseases - which account for the vast bulk of U.S. healthcare costs.

\section{REFERENCES}

1. Cook DJ, Krishnan N. Mining the home environment. J Intell Inf Syst 2014; 43(3):503-19.

2. Zullig LL, Melnyk SD, Goldstein K, et al. The role of home blood pressure telemonitoring in managing hypertensive populations. Curr Hypertens Rep 2013; 15(4):346-55. 
3. Adler-Milstein J, Sarma N, Woskie LR, et al. A comparison of how four countries use health IT to support care for people with chronic conditions. Health Affairs 2014; 33(9):1559-66.

4. Paré $G$, Jaana $M$, Claude Sicotte $C$. Systematic review of home telemonitoring for chronic diseases: the evidence base. J Am Med Inform Assoc 2007; 14(3):269-77.

\section{Objectives}

1. Discuss the utility of a personalized, rules-based, machine-learning analytics system using home monitoring data combined with other longitudinal healthcare data to reduce avoidable acute care events (such as hospitalization or emergency department)

2. Predict which patients are more likely to benefit from home monitoring

3. Explain how day-to-day changes and longer-term trends in measurements of multiple parameters compared to a patient's moving baseline detect patterns that predict deterioration days in advance of the patient becoming severely symptomatic

\section{TUESDAY, MAY 17, 2016}

3:00 PM-4:00 PM Tuesday, May 17, 2016

\section{Session 64 Session Title: MARKET WATCH}

MODERATOR: Victor Camlek, Principal Analyst, Transformational Health | Connected Health

Frost \& Sullivan, New York, NY

PRESENTER: Sheri Dodd, MSc, Vice President and General Manager Medtronic Care Management Services, Medtronic, Chanhassen, MN

PRESENTER: Jason Gorevic, Chief Executive Officer Teladoc, New York, NY

Frost \& Sullivan will host a Market Watch panel focused on two segments of telehealth that are expected to take off over the next five years. A panel of experts will address anticipated market developments in Clinical Grade Remote Patient Monitoring and the Interactive Virtual Telemedicine markets. This interactive session will engage and poll the audience using the conference mobile app.

\section{Objectives}

1. Review leading opportunities and challenges

2. Gain valuable insights from leading market experts

3. Identify factors to address in strategic planning efforts

\section{Track: Clinical Services Room 102 D}

\section{SUNDAY, MAY 15, 2016}

12:00 PM-1:00 PM Sunday, May 15, 2016

\section{Session 4}

Session Title: INNOVATIVE WAYS TO USE TELEMEDICINE TO TREAT INFECTIOUS DISEASES

PRESENTER: Jawad Nazir, MD, FACP, Physician, Infectious Disease Infectious Disease Specialists PC, Sioux Falls, SD

PRESENTER: Tammy Hatting, MPA, eCARE Innovation Manager Avera Health - eCARE, Sioux Falls, SD

The challenges of access to medical care can present a barrier to good health whether people live in urban or rural areas. That's especially true for health systems that cover hundreds or thousands of square miles including sparsely populated rural regions. With healthcare specialists and subspecialists often concentrated in urban centers, rural providers and residents face challenges with timely access to adequate care where they live. Nearly $20 \%$ of people living in rural or remote regions say they would not seek medical care if it meant a long drive, time away from work or the expense of travel and outof-town lodging. Many don't have the support network of family and friends to help them get care far from home. Or weather-related challenges may affect their ability to get care. For healthcare providers, drive time needed to reach outlying clinics could be better spent caring for more patients close to home. When specialists implement eConsult, all of the communities they serve gain access to non-urgent medical specialty care through interactive video technology located at their local facility.

By conducting the actual visit through interactive video technology, patients save the time and expense of long-distance travel to the specialty provider. That means they're more likely to get the medical care they need. In fact, 98\% of patients who responded to the Avera eCARE surveys report high satisfaction with their eConsult visits. Payers have also recognized the value of eConsult services. In many cases, Medicare and other payers reimburse for this care at the same level as in-person services. Services are billed using several of the same office visit codes used in traditional practice. This allows for seamless healthcare delivery to patients in rural and underserved areas. By using eConsult services, facilities and providers help boost local economies by retaining ancillary charges and other healthcare revenue in rural clinics.

Currently, the Infectious Disease specialty represents over $60 \%$ of the volume for the eConsult program at Avera Health, where each month, they average 400-500 visits in approximately 67 locations which include clinics, hospitals, long term care facilities and correctional institutions. In addition to office visits, infectious disease specialists are creating the vision for an eAntimicrobial Stewardship Program (eASP) to create a centralized focus within the Avera system to limit the overuse and misuse of antibiotics, optimize clinical outcomes and reduce the emergence of resistance. A growing body of evidence demonstrates that hospital-based programs dedicated to improving antibiotic use can both optimize the treatment of infections and reduce adverse events associated with antibiotic use. The eASP program is an Avera Health collaborative quality initiative led by an infectious disease physician specialist and supported by an infection control pharmacist utilizing technology and EMR systems to evaluate inpatient usage of broadspectrum antibiotics. Increasing antimicrobial resistance is one of the world's most pressing public health threats so there is a need for consistency and standardization throughout the system utilizing evidence-based guidelines. The appropriate usage of antibiotics will significantly reduce costs and the adverse effects associated with antimicrobials which result in more visits to the emergency room than any other drug classes, such as anticoagulants. Telemedicine services will be utilized for relevant data collection (patient history, microbiological data, antimicrobial use, etc), analysis, recommendations, education and consults. Infectious disease consultants in collaboration with pharmacist and infection prevention will determine rules which will help in identifying interventions in a timely manner to achieve overall goals of the eASP program. This may require review of hospital antibiograms, antimicrobial utilization data and antimicrobial resistance trends. These may differ from facility to facility and antimicrobial stewardship interventions may need to be selected based on these reviews. This session will discuss the importance of an eConsult program and demonstrate the possibilities and innovative experience of Avera eCARE and Infectious Disease PC in initiating quality programs utilizing telemedicine.

\section{Objectives}

1. Understand the value of telemedicine in the treatment of infectious diseases in rural hospitals and clinics

2. Discuss the impact of the infectious disease telemedicine consultations in decreasing antimicrobial resistance

3. Share the importance of collaboration and teamwork in developing a successful infectious disease telemedicine program 
SUNDAY, MAY 15, 2016

1:15 PM-2:15 PM

Sunday, May 15, 2016

\section{Session 12 \\ Session Title: USING VIDEO TECHNOLOGIES TO IMPROVE INFECTIOUS DISEASE MANAGEMENT: A CASE STUDY OF TUBERCULOSIS}

MODERATOR: Dale Alverson, MD, Professor Emeritus of Pediatrics \& Regents' Professor

University of New Mexico, Albuquerque, NM

PRESENTER: Mario Gutierrez, MPH, Executive Director

Center for Connected Health Policy, Sacramento, CA

PRESENTER: Lois Ritter, EdD, MS, MA, MS-HCA, Consultant

Consultants in Health, Alameda, CA

Introduction: Limited studies have been conducted on using telehealth to treat and prevent the spread of infectious disease. Tuberculosis (TB) is one of the deadliest infectious diseases in the world, causing over 8.8 million people to become ill and 1.4 million people to die annually from the disease (WHO, 2013). $\mathrm{TB}$ is treatable, however, poor adherence to daily medication regimens lasting 6-24 months leads to ongoing disease transmission, higher mortality, and acquisition of antibiotic resistant TB. "Directly observed therapy" (DOT) is recommended by the Centers for Disease Control and Prevention (MMWR, 2003 ) to minimize these problems by having healthcare workers (HCWs) watch patients take each dose of medication. While proven effective for improving adherence, DOT is costly, time consuming, potentially invasive, and often impossible for patients who need to take their medications outside of normal business hours or when they travel. Thus, some TB programs have begun using landline and mobile phones to conduct video DOT (VDOT). With VDOT, patients can schedule a time to meet with HCW using live video (synchronous VDOT) or the patient can record him or herself taking the medication and forward the video to the HCW (asynchronous VDOT). VDOT allows patients to take their medications at their preferred location and avoids the need for either party to travel. However, due to a lack of empirical evidence demonstrating that VDOT is comparable to traditional in-person DOT, health officials are wary of incorporating this technology solution into their TB programs. Other barriers to adoption of VDOT include the lack of guidance from health agencies on the use of VDOT, health policies that do not allow health departments to be reimbursed for these services and other legal and regulatory barriers. The problem is how to overcome these barriers in order for TB care providers to adopt disruptive technologies such as VDOT.

Objectives \& Methods: Collaborators from the Center for Connected Health Policy (CCHP), the University of California, San Diego (UCSD), and five California county health departments formed an academic, public health and health policy partnership to increase access to telehealth for treating TB and potentially other infectious diseases. UCSD researchers conducted epidemiological studies to evaluate the acceptability and efficacy of the VDOT system through local health departments to inform guidance documents and policy papers for using VDOT. These studies involved measuring medication adherence among $270 \mathrm{~TB}$ patients who used VDOT in rural and urban counties to patients who received in-person DOT. The public health partners utilized VDOT for their TB patients and developed an agency guidance document to assist other local health jurisdictions in adopting VDOT. CCHP conducted a literature review, interviews with key health professionals working at the pilot sites, examination of existing state and federal laws, and a survey with TB experts. Results: The findings to date show that video-DOT is a viable option for providing DOT to TB patients. Multiple benefits are perceived or experienced such as patient and staff satisfaction, cost effectiveness, staff safety, and improved medication adherence. The highest perceived or experienced concerns are related to reimbursement, start-up costs, connectivity problems, and equipment problems. The financial concerns stem from the fact that public and private payers do not reimburse for video-DOT. When asynchronous and synchronous Tele-DOT are compared, the perceived and experienced concerns and benefits were very similar. The greatest difference was a lower reported benefit of managing side effects with asynchronous when compared to synchronous. Users of VDOT have fewer concerns than non-users and reported greater benefits overall. Overall, 89\% of patients reported never/rarely having problems recording videos, 92\% preferred VDOT over in-person DOT, 81\% thought VDOT was more confidential, and 100\% would recommend VDOT to others. Analysis and interviews indicated that while there is great interest in using VDOT, reimbursement from private and public payers did not exist. A VDOT Guidelines working group was sanctioned by the California TB Controllers Association, which has begun drafting the document.

Conclusions: Preliminary data suggest that VDOT provides a promising mobile solution to the high cost and burden of in-person DOT for monitoring $\mathrm{TB}$ and other conditions that require strict treatment adherence. The benefits are numerous, but what is keeping video-DOT from widespread expansion is primarily related to technology and finances. Connectivity, equipment, and security problems are the technological concerns and lack of reimbursement and start-up costs are the financial concerns. It is anticipated that the technology problems will be reduced with the advances in the field, but policy changes related to reimbursement are needed to alleviate the financial barrier.

\section{REFERENCE}

1. Treatment of Tuberculosis. MMWR Recommendations and Reports, June 20, 2003 / 52(RR11);1-77.

\section{Objectives}

1. List at least four advantages and two disadvantages of VDOT for monitoring anti-TB medication adherence

2. Explain at least three relevant policies to the utilization of VDOT

3. Describe the efficacy and patient satisfaction of VDOT compared to inperson DOT

\section{MONDAY, MAY 16, 2016}

8:00 AM-8:45 AM Monday, May 16, 2016

\section{Session 20}

\section{Session Title: CASE STUDIES IN TELECARDIOLOGY}

MODERATOR: Earl W. Ferguson, MD, PhD, FACC, FACP, FACPM, Medical Director, Chief Medical Officer

Ridgecrest Regional Hospital, Radekal/Pertexa, Ridgecrest, CA

INNOVATIVE TELEMEDICINE USE IN A HEART FAILURE PAY

FOR PERFORMANCE MODEL

PRESENTER: Brenda L. Bartock, MPA, BSN, RN, Telehealth Clinical Project Director

University of Rochester Medical Center, Rochester, NY

The healthcare community must develop new care delivery models that will allow them to be financially viable in the evolving pay for performance environment. The University Of Rochester Medical Center entered into the Medicare Bundled Payments for Care Improvement (BPCI) Initiative for heart failure beginning July 1, 2015. The goal of their model is to achieve higher quality and more coordinated care at a lower cost to Medicare. The key to the model is use of telemedicine for remote cardiology visits.

A bundle is a single budget for a defined group of services associated with a medical or surgical condition. It covers all associated services during a spec- 


\section{ORAL PRESENTATIONS ABSTRACTS}

ified period of time and covers a defined period of time (e.g. 30, 60, 90 days post discharge). The University Of Rochester Medical Center's Medicare bundle begins at admission where the primary discharge DRG is CHF (MS-DRG 291, 292, 293). It includes all Part A and B services received during the inpatient stay and for a 90 day period after discharge (no matter where the services are provided-inpatient, outpatient, SNF, home health, etc.). The hospital is at risk for a budget based on trended historical expenses less than $2 \%$.

The key resources and processes include:

- Clinical pathways for inpatient and post-acute services

- A dashboard built into the electronic health record that tracks the patient throughout the episode

- A Care Navigator who has primary responsibility for following the patient and coordinating services and transitions

- An enhanced home care component that includes innovative use of telemedicine for not only biometric monitoring, but also early followup virtual appointments with cardiology and rapid response virtual consults with cardiology that may result in administration of intravenous medication to remove excess fluid to avoid readmission

- Financial tracking in "real" time

- Multi-disciplinary team overseeing the program

The use of telemedicine for virtual cardiology appointments with patients is a new innovation in our community in the care of patients with heart failure. The Information Services Division of the medical center took lead responsibility in working with the clinical staff to find a solution that met the rigorous quality standards necessary for a virtual versus a face to face assessment. A platform for secure videoconferencing and an electronic stethoscope were tested and selected. The Cardiology department was very pleased with the result. Important in this process was the fact that the lead Cardiologist was very comfortable with technology and supportive of the use of telemedicine applications in the management of heart failure.

All patients receiving home care also received biometric monitoring. Select patients are scheduled at home or at SNFs for virtual cardiology appointments on day 3 post discharge from the hospital. Lastly, the virtual visits are done in conjunction with a home care nurse or SNF staff using videoconferencing equipment and electronic stethoscopes. This allows for transmission of heart and lung sounds as well as visualization of the body for fluid accumulation. Vital signs/biometric trends are faxed in advance of the virtual appointment in addition to the cardiology staff having access to the home care telehealth portal for their patients. Finally, there is the ability to do urgent virtual consults for acute exacerbations or changes in condition where the cardiologist or designated provider may prescribe an intervention (such as oral diuretics, intravenous diuretics, oxygen adjustments, etc.). Having the home care nurse or SNF staff present with the patient during the virtual visit allows for improved communication across the continuum of care. There is multistakeholder investment in the use of the telemedicine intervention. In addition, the virtual visit provides the clinical context for the collected biometric data, enabling clinicians to better determine the most appropriate treatment.

This three year risk arrangement with Medicare for heart failure has just begun. Data will be evaluated and presented looking at resource utilization in the 90 day post discharge period, readmission rates and emergent care use, costs and patient/provider satisfaction with care. Expected outcomes include a reduction in emergent care and readmissions, cost of care reduction meeting the bundle targets, and high patient and provider satisfaction with care. Data is not currently available but there will be a number of 90 day episodes completed by the time of the ATA presentation that will be shared and compared with 2014 results.

\section{REFERENCES}

1. Aston G. (2015, March 10). Telehealth Promises to Reshape Healthcare: Hospitals Embrace Powerful New Tools to Continuously Connect to Patients. Retrieved from http://www.hhnmag.com/Magazine/2015/Mar/cov-telehealth patients-connect
2. Idris S, Degheim G, Ghalayini W, Larsen T, Nejad D, David S. Home Telemedicine in Heart Failure: A Pilot Study of Integrated Telemonitoring and Virtual Provider Appointments. Rev Cardiovasc Med 2015; 16(2):156-62.

3. American College of Cardiology (2013, Nov 26). Partners in Innovation: Program Finds HF Improvements with Virtual Connections. Retrieved from http://www.acc.org/latest-in-cardiology/articles/2013/11/26/09/49/partners-ininnovation-program-finds-hf-improvements-with-virtualconnections\#sthash.NvMvrkDL.dpuf

4. Joynt KE, Jha AK. A Path Forward on Medicare Readmissions. N Engl J Med 2013; 368(13):1175-177.

\section{Objectives}

1. Demonstrate awareness of the importance of investigating the application of telemedicine technologies in the evolving pay for performance environment of healthcare to achieve the triple aim

2. Identify the processes necessary in implementing a telehealth solution that involves multiple service providers in the care of a patient

3. Evaluate the potential value proposition of a multi-stakeholder telemedicine initiative to reduce readmissions in the heart failure patient population

\section{IMPROVING ACCESS TO CARDIOLOGY SERVICES AT REGIONAL VA MEDICAL CENTERS THROUGH TELEMEDICINE}

PRESENTER: Stephanie L. Deaner, PhD, VISN Telehealth Program Manager $V A$, Westchester, IL

Telespecialty care involves providing care from a tertiary medical center where a specialty care provider is on staff to a smaller medical center, where there is a shortage of specialty care providers and/or services. VA Medical Centers (VAMCs) in the mid-west are striving to provide a full range of telehealth services to the veteran population in specialty care. One of these specialty areas is cardiology.

The mid-western VAMCs are a pioneer of telecardiology. In 2010, two mid-western VAMCs decided to expand telespecialty and telemedicine services to rural veterans. A rural telehealth initiative required the collaboration of a tertiary VAMC located in Milwaukee, Wisconsin in order to reach Veterans residing in rural and highly rural counties in the upper peninsula of Michigan. Since the VAMC in Iron Mountain, Michigan had limited cardiology staff, the appointment is conducted via telehealth, in order to limit veteran travel. Drive times for veterans to the referral VAMC in some mid-western areas can take up to 7 hours. Offering telecardiology services to veterans at their local VAMC or CBOC expands their access to cardiology care and reduces travel time.

Since 2010, as resources became available, expansion of more telehealth services proceeded throughout the region providing telecardiology care to additional CBOCs in rural and non-rural areas. The expansion of telecardiology care continues to the present. Telecardiology is one of the most developed telespecialty care programs within the mid-west.

For the past half-decade, the mid-west has been the national leader in the VA for number of veterans served via telecardiology, primarily due to the program between the VAMCs in Iron Mountain, Michigan and Milwaukee, Wisconsin. The telecardiology program has exponentially grown from 17 patients in 2010 to over 700 patients each in 2014 and 2015.

Currently at five VAMCs in the mid-west, Cardiology Telehealth Clinical Applications include care between two or more VA Medical Centers (VAMCs) or between a VAMC and Community Based Outpatient Clinics (CBOCs). The purpose of telecardiology is the delivery of specialized cardiac care, such as for patients with chest pain, arrhythmias, and valve disorders, between VA centers of specialized care and patient sites lacking that expertise.

This presentation will focus on the necessary tools for a successful telecardiology program. It will discuss equipment, staff training, risk management, quality management, and lessons learned. 
All requests for telecardiology services will be reviewed by the consulting team at the tertiary medical center who will make the determination whether the visit is appropriate for telehealth or if the patient needs a face-to-face visit. Reasons for telecardiology referrals include, but are not limited to: ischemic heart disease, atrial fibrillation, congestive heart failure, post-cardiac surgery, ventricular arrthymias, prosthetic valves, syncope, and valvular heart disease.

For a telecardiology appointment to be successful, the staff need to complete training on telehealth and the equipment. The cardiologist needs a monitor with a codec and a stethoscope. Ideally, the codec should provide the ability to allow for far end remote control. At the patient site, there is a staff member, usually a health technician or Licensed Practical Nurse, with the patient to operate the telehealth equipment. The equipment at the patient site is a telehealth cart and codec, with a camera that can pan, tilt, zoom and has far end remote control capabilities.

The use of peripherals during the telecardiology visit is perhaps the most integral part of the telehealth encounter. By using a telestethoscope receiver, cardiology providers are able to receive high quality, real-time heart sounds and other internal sounds of the body. Telemedicine carts at the patient site are equipped with send units for the telestethoscope.

Risk management for telecardiology is twofold: effectively managing a medical or mental health emergency and managing equipment failure that prevents the patient and cardiologist from doing telecardiology. Emergency procedures must be in place prior to an acute event as well as a plan for telehealth equipment failure.

Lastly, telecardiology programs should incorporate performance improvement and quality management. Program evaluation planning should be performed along with telecardiology program development and deployment. The focus can be on clinical, business, and technical areas.

\section{Objectives}

1. Identify risk management factors involved in implementing a telecardiology program

2. Apply performance improvement factors involved in a telecardiology program

3. Demonstrate awareness of the equipment and training needed for a telecardiology program

\section{MONDAY, MAY 16, 2016}

11:00 AM-12:00 PM

Monday, May 16, 2016

\section{Session 28 \\ Session Title: INTEGRATED EHR AND TELEMEDICINE CARDIOVASCULAR MANAGEMENT}

MODERATOR: Neal Sikka, MD, Section Chief, Innovative Practice and Telehealth Section

The George Washington - Medical Family Associates, Washington, DC

USE OF MY HEALTHEVET - THE VETERANS AFFAIRS ELECTRONIC HEALTH RECORD (EHR) - FOR MANAGEMENT OF HEART FAILURE

PRESENTER: Stuti Dang, MD, MPH, Staff Physician ${ }^{1,2}$, Diana I. Ruiz, BSN ${ }^{1}$, Andrew L. Sorial, $\mathrm{MD}^{1}$, Carlos A. Gomez-Orozco, BS ${ }^{1}$, Kris Siddharthan, $\mathrm{PhD}^{3}$, Orlando W. Gomez-Marin, $\mathrm{PhD}^{2}$, Remberto Rodriguez, $\mathrm{MD}^{1}$

${ }^{1}$ MIAMI VA Hospital, Miami, FL, ${ }^{2}$ University of Miami Miller School of Medicine, Miami, FL, ${ }^{3}$ Tampa VA Hospital, Tampa, FL

Introduction: In 2009, President Obama signed into law the Health Information Technology for Economic and Clinical (HITECH) Act. This Act offered numerous incentives which aimed not only for widespread implementation of electronic health records, but also for its "meaningful use" in primary care settings. An electronic health record is "a digital version of a patient's paper chart...patientcentered records that make information available instantly and securely to authorized users". Many electronic health records give patients access to their health records and allow them to exchange messages with their providers, which are potential features that can be maximized and may offer clinical efficiencies. For healthcare systems that have already adopted electronic health record systems with secure messaging, this tool will be available at no additional cost.

Purpose: We implemented an educational, case management, and monitoring intervention for patients with heart failure, using the Veteran Affairs' comprehensive patient portal, My HealtheVet, and its secure messaging feature. Patients were sent weekly information on managing their heart failure. In addition, they were asked questions related to weight, medication adherence, and heart failure symptoms every week. Responses were tracked for possible deterioration in heart failure. Positive responses for increase in weight, and worsening symptoms were communicated to the primary care providers and interventions were made per primary care recommendation. This was a feasibility and proof of principle study. Relevant telehealth implementation barriers were identified.

Methods: The project enrolled $118 \mathrm{HF}$ patients and instructed them on how to navigate My HealtheVet and reply to secure messages. Usability data was obtained from the 118 participants; their average age was $65.1 \pm 9.6$ years, ranging from 41-91 years. A total of 103 (87.3\%) were non-Hispanic, and 15 (12.7\%) were Hispanic or Latino; included were 90 (76.3\%) Whites, and 20 (16.9\%) African Americans. Sixty-two (53\%) were married, 29 (25\%) divorced; median yearly income was $\$ 20,292$. The Veterans were asked to log onto My HealtheVet weekly and report their weight and symptoms common to heart failure such as shortness of breath, weight gain, or swelling of the legs. They also received weekly short educational messages related to heart failure. Patients' perceptions, concerns and general satisfaction were also explored.

Results: Initial monitoring of enrolled participants lasted 26 weeks. Among the 118 participants, 55 (46.6\%) used MHV between 1 to 26 weeks. Their response rate averaged $46.6 \pm 29.6 \%$, and ranged from $5 \%$ to $100 \%$. No participants were withdrawn for nonresponse. Out of the 55 participants who ever responded, 17 (31\%) responded $<25 \%$ of the weeks, 12 (22\%) responded $25-50 \%$ of the weeks, $16(29 \%)$ responded $50-75 \%$ of the weeks, and $10(18 \%)$ responded $>75 \%$ of the weeks. A total of 26 (47\%) participants responded at least 50\% of the weeks they were enrolled. Among the 55 responding, the number of response weeks ranged from 1 to 26 with a median of 8 and a mean of $9.4 \pm 7$. Three questionnaires were administered at baseline, and 3 months follow-up, to evaluate quality of life $(\mathrm{QoL})$, patient knowledge, and chronic disease selfefficacy. There was no significant change in QoL based on the Minnesota Living with Heart Failure $(p=0.128)$, or Chronic Disease Self-Efficacy (Stanford Questionnaire) $(\mathrm{p}=0.256)$. There was, however, a statistically significant improvement in patient's heart failure knowledge as measured by the Dutch Heart Failure Knowledge Scale ( $p=0.011)$. Seventy-four participants responded to our follow-up survey, (50) 67\% agreed that they had improved their ability to take care of their health, and (47) 64\% now had access to heart failure information that would otherwise have been difficult to obtain. Sixty-four percent (47) found it easy to communicate with the project team and only (7) $9 \%$ thought that using secure messaging was too difficult.

Conclusions: Electronic health records based interventions are a feasible way to improve patient knowledge. Usability and technical issues faced by participants can likely be overcome by additional training sessions for both patients and staff. As of yet, there is no distinct strategy for keeping patients engaged in self-management, but as new electronic health record technology continues to emerge and existing electronic health record systems improve, they may offer another feasible modality to enhance patient care. Special attention is warranted in order to make electronic health records more user friendly in order to improve patient usage. Further studies need to be done to assess clinical significance, and to determine how its potential can be best harnessed. 


\section{REFERENCES}

1. "Heart Failure Fact Sheet." Centers for Disease Control and Prevention. Centers for Disease Control and Prevention, 3 Dec. 2013. Web. 10 Nov. 2014. retrieved from http://www.cdc.gov/dhdsp/data_statistics/fact_sheets/

fs_heart_failure.htm

2. Purcell $R$, Mclnnes $S$, Halcomb EJ. Telemonitoring can assist in managing cardiovascular disease in primary care: a systematic review of systematic reviews. BMC Fam Pract 2014; 15(1):43.

3. Paré $\mathrm{G}$, Jaana $\mathrm{M}$, Sicotte $\mathrm{C}$. Systematic review of home telemonitoring for chronic diseases: the evidence base. J Am Med Inform Assoc 2007; 14(3): 269-77.

\section{Objectives}

1. Explore innovative strategies for heart failure management

2. Assess feasibility of using EHR for chronic disease management

3. Evaluate the impact of EHR use on self-efficacy and quality of life in patients with heart failure

STUDY OF EFFICACY OF INTEGRATED EHR AND TELEHEALTH SYSTEM IN COORDINATED CARDIOVASCULAR CARE IN REMOTE CRITICAL ACCESS HOSPITAL

PRESENTER: Earl W. Ferguson, MD, PhD, FACC, FACPM, Medical Director, Kishor Joshi, MBA, Russ Rudin, PhD

Pertexa/Radekal, Ridgecrest, $C A$

Background: An integrated, interoperable EHR/HIE/HIT/telehealth system has been developed to markedly improve physician and other health provider visit productivity. This system allows expedited visits using touch tablets that do not require typing or dictation to document histories and physical examinations coupled with almost 4000 anatomic diagrams linked with diagnostic algorithms based on chief complaints that lead providers though the normal clinical exam process. The tablet anatomic diagrams can be reviewed with the patient to determine and clarify exact diagnoses - for example specific shaded areas of chest pain can be identified specifically by the patient for the provider to document with a touch of the stylist, and then specific areas of radiation of that pain can similarly be documented; as another example, muscle, tendon, joint and bone discomfort can similarly be evaluated through different layers of anatomy to specify and educate the patient about their problem. Diagnoses are then identified and can be linked to e-Prescriptions, laboratory and imaging studies, and on-demand telecardiology and other consult support services. Once the visit is complete, a natural language generator provides a detailed text visit note that documents the visit. Coding is automatically linked to diagnoses. Once the text visit note is reviewed, the provider can sign the note and the visit can be completed, billed and orders sent before the patient leaves the examining room.

Objectives: The efficiency and effectiveness of this integrated system is being documented and will be evaluated with an independent review by the University of Southern California Keck School of Business.

Findings: Preliminary estimates are that this system decreases the time for completion of a provider visit by $30-50 \%$ over standard EHRs that require typing and/or dictation.

Conclusions: This integrated, interoperable EHR/HIE/HIT/telehealth system is a disruptive technology that improves provider productivity, decreasing the time required for a visit and markedly improving interactions with patients.

\section{REFERENCE}

1. Ferguson EW. (2014) American Healthcare Reform: Fixing the Real Problems. Bloomington, IN: AuthorHouse.

\section{Objectives}

1. Understand value of integrated, interoperable systems

2. Ways to markedly improve efficiency

3. Value of patient education with visits

\section{MONDAY, MAY 16, 2016}

\section{Session 34 Session Title: REVOLUTIONIZING SEXUAL ASSAULT CARE: DEVELOPING THE NATIONAL TELENURSING CENTER TO SERVE POPULATIONS IN NEED}

MODERATOR: Cynthia A. Forbes, RN, MSN, CEN, NREMT-P, SANE, Sexual Assault Response Team Coordinator

Suttler Lakeside Hospital, Lakeport, CA

PRESENTER: Stacy E. Garrity, RN, MS, WHNP-BC, SANE, Director National TeleNursing Center, Newton, MA

PRESENTER: Joan Meunier-Sham, RN, MS, Director MA SANE Program, Boston Medical Center, Boston, MA

Adult and adolescent sexual assault victims have unique medical, emotional and forensic needs. Sexual Assault Nurse Examiner (SANE) programs have demonstrated improved quality of healthcare for patients, increased quality of forensic evidence collection, and increased success with prosecution. However, access to SANE programs and other medical forensic expertise is not uniformly available throughout the country, particularly in remote and rural areas. Healthcare professionals (nurses, nurse practitioners, physicians, and physician assistants) trained to conduct forensic sexual assault exams without SANE expertise often do not see the volume of patients required to maintain proficiency in examination and evidence collection technique. Additionally, healthcare providers often lack the emotional support and ongoing education required for this work leading to high attrition rates. Telemedicine holds promise as a vehicle for increasing clinician's aptitude for providing care to this unique patient population and expanding access to forensic nursing expertise across the United States.

Following a national solicitation, the Massachusetts SANE program received a \$3.2 million grant from the Office for Victims of Crime in collaboration with the National Institute of Justice to establish a telenursing center to support clinicians in four unique populations across the country as they provide care to recent adult and adolescent victims of sexual assault. The populations identified, (military, tribal, rural, and corrections), demonstrated a need for additional support to bolster the care they are providing within their communities. Collaborating with an established SANE program via telemedicine provides an opportunity to introduce or enhance services for survivors at healthcare facilities within the identified populations.

This three year federally funded demonstration project has progressed though the planning phase and is currently providing 24/7 support and guidance to healthcare providers across the country as they care for recent victims of sexual assault, utilizing telemedicine technology and best practice nursing standards. Despite the challenges and obstacles, successful partnerships are established and patients are receiving collaborative care from expert SANEs in this pilot project.

\section{REFERENCE}

1. A Renewed Call to Action to End Rape and Sexual Assault. http://www.whitehouse .gov.sites/default/files/docs/sexual_assulat/report_1-21-14.pdf 


\section{ORAL PRESENTATIONS ABSTRACTS}

\section{Objectives}

1. Discuss the primary obstacles overcome by collaborating agencies to develop the National TeleNursing Center and the established care delivery model

2. Identify the challenges of supporting professionals as they provide victim centered forensic nursing care to patients in healthcare facilities remote from expert SANEs

3. Illustrate the interactive nature of a TeleNursing Encounter, the process of providing and receiving real-time guidance, and discuss three-case studies to exemplify the nature of the experience

\section{MONDAY, MAY 16, 2016}

4:15 PM-5:15 PM

Monday, May 16, 2016

\section{Session 41 Session Title: IS TELEMEDICINE GOOD ENOUGH?}

MODERATOR: Michael V. Bess, MD, MHA, National Medical Director Telehealth

Optum, Galena, $\mathrm{OH}$

PRE-OPERATIVE TELEMEDICINE EXAMINATION IS EQUALLY AS SAFE AND EFFECTIVE AS IN-PERSON PREOPERATIVE EXAMINATION BASED ON A LONGITUDINAL CASE-MATCHED SERIES OVER 4 YEARS

PRESENTER: Andrew R. Watson, MD, MLitt., FACS, Vice President, International Division, Medical Director, Telemedicine UPMC, Pittsburgh, PA

Preoperative evaluation of patients using telemedicine (TM) is becoming increasingly important for patients in rural and underserved areas to access critical subspecialty procedural care. Patients in such areas frequently are unable to drive due to medical conditions, are limited by weather, have to pay out of pocket for travel expenses and cannot take time off work to travel for surgical specialists. Therefore, telemedicine has the potential to save patient's time and money and give them access to medically necessary procedural care without barriers of cost, travel and time lost.

Between March 6, 2000 and July 18, 2014, a single colorectal surgeon first met and evaluated 30 patients using TM in rural / underserved areas of Pennsylvania. All 30 patients were brought to the urban tertiary center for surgery. The patients were first met face-to-face in the preoperative holding area by the surgeon. The patient surgical outcomes and preoperative comorbidities were compared to 308 non-telemedicine control patients who were seen in person (IP). Outcome measures focused on complications, total case time, set-up time, DRG weight, length of stay, number of post-op emergency department visits and readmissions. These are core metrics of surgical success and were used to measure the efficacy of TM only versus IP preoperative assessment. There were no statistical differences in all core metrics between the telemedicine and in-person preoperative evaluation, with the only trend that telemedicine patients were more often likely to return to the $\mathrm{OR}(\mathrm{p}=0.70,3 \% \mathrm{vs} 10 \%)$. There was not a high readmission rate ( $9 \%$ IP vs $10 \%$ TM) or length of stay ( $3.9 \%$ IP vs $5.2 \%$ TM). Complications were unaffected by telemedicine, $23 \%$ IP vs. $20 \%$ TM. Procedure time, total case time and set-up time were all not significantly different.

Overall, this study shows that a preoperative evaluation with telemedicine is safe and effective as it does not lead to a higher complication rate, readmission rate, ED utilization or unfavorable operating room metrics. Telemedicine can be offered to patients as a preoperative evaluation modality and does not disrupt core urban operation room function.

\section{Objectives}

1. Understand the safe evaluation of preoperative patients using telemedicine

2. See the financial and clinical outcomes of telemedicine preoperative evaluation

3. Understand how a quality based study in telemedicine is conducted

COMPARISON OF IMAGES GENERATED BY SMARTPHONES FOR USE IN TELEPATHOLOGY

PRESENTER: William Eng, MD, Assistant Professor ${ }^{1}$, John Barksdale, BS ${ }^{2}$, Robert Norman, D0 ${ }^{1}$

${ }^{1}$ University of Central Florida Medical School, Orlando, FL,

${ }^{2}$ Pathology Services LLC, North Platte, NE

Introduction: Pathologists have for many years embraced the utility of photography in their practice. Less than 20 years ago, film photography was the dominant method of capturing images under a microscope. Microscopes arranged specifically for photography were outfitted with a "triocular" head reserved for a camera attachment, usually a $35 \mathrm{~mm}$ SLR. The film could be processed for Kodachrome or printed for publications. However, over the past 15 years, fixed digital cameras have largely supplanted the use of film. A separate evolution has been the incorporation of digital cameras within cell phones, but these early models produced low resolution and amateurish photos. However, the Apple's iPhone 5 has a 8-megapixel camera, and the Samsung's Galaxy 6S phone has a 16megapixel camera. For pathologists, the primary question is whether the camera can produce a photograph which he/she can view and arrive at a diagnosis that is the same as if the specimen was being examined under a microscope. This "validation" process has even been incorporated into the FDA's proposal rules concerning telepathology.

Methods: 25 skin specimens that were collected in a private dermatology office were randomly selected for "Quality Assurance" review and photographed using an iPhone 5 and Galaxy 6S smart phones. Photos were taken at 40,100 , and $400 \times$ magnifications by bringing the smart phone camera adjacent to the right eyepiece of a Nikon Labophot-2 microscope. The Nikon Abbe 125 condenser was set at 15, while the lamp intensity was set to 1.2. Both phones took photos of the identified field of view so that a direct comparison can be made.

Results: Both the iPhone 5 and Galaxy $6 \mathrm{~S}$ smart phones produce images acceptable for diagnosis. In addition, the images were of sufficient resolution for a pathologist to arrive at a diagnosis comparable to the original diagnosis. The color of the images from both smart phones were a close approximation to the true color. Images that contained more than $60 \%$ white background were found to be slightly poorer contrast, so images at higher magnifications that filled up the field of view often appeared sharper. The autofocus feature worked exceptionally so even images that were purposely out of focus slightly could be corrected by the camera software.

Conclusions: Quality assurance cases that were previously examined by 2 independent, board certified pathologist who arrived at the same diagnosis were used to eliminate interpretation variations. Optimal positioning of the camera directly in front of the eyepiece required steady hands. While multiple models of cellphone holders are commercially available, this study did not employ them. These devices do aid in holding the camera steady at a fixed angle and focal distance, but they are configured for smart phones without a case. This becomes a cumbersome proposal to remove the phone from its casing every time the camera is used. Another issue is that the added weight on the microscope's eyepiece which could cause excessive wear on its threads. A fixed spacer between the eyepiece and camera would have to be developed for reproducible focal length. Since the camera's white balance is automatic, photographing minute 


\section{ORAL PRESENTATIONS ABSTRACTS}

specimens at low magnification may be problematic. The importance of properly processed, cut, and stained tissue cannot be diminished since poor quality tissue makes it difficult to render an accurate diagnosis regardless of the camera system. Finally, the operator's level of experience creates a hidden bias. Whereas a more experience pathologist can zoom in a specific feature of the diagnosis, others may not be able to identify these salient features as readily. In our experience as well as that of others, the technology of current smartphone cameras are capable of producing images that are of sufficient technical quality for a pathologist to make an accurate diagnosis. However, transmission of patient images over an unsecure network is a risk for HIPPA violation.

\section{REFERENCES}

1. Clinical Guidelines for Telepathology, May 2014. American Telemedicine Association.

2. Fontelo P. et al. Evaluation of a cellphone for telepathology: Lessons learned. J Path Inform 2015; (6):35

3. Farahani N, Pantanowitz L. Overview of Telepathology. Surg Pathol Clin 2015; $8(2): 223-31$

\section{Objectives}

1. Understand the current ability of smart phone cameras to image pathology slides

2. Know the limitation of taking photos using smartphone cameras

3. Recognize the factors which contribute to high quality images

\section{EVALUATION OF SYMPTOM CHECKERS FOR SELF-DIAGNOSIS} AND TRIAGE: AUDIT STUDY

PRESENTER: Hannah Semigran, BA, Research Assistant ${ }^{1}$, Jeffrey Linder, MD, $\mathrm{MPH}^{1,2}$, Courtney Gidengil, MD, $\mathrm{MPH}^{3,4}$, Ateev Mehrotra, MD, MPH, MS ${ }^{1,5}$

${ }^{1}$ Harvard Medical School, Boston, MA,${ }^{2}$ Brigham and Women's Hospital, Boston, MA,${ }^{3}$ RAND, Boston, MA, ${ }^{4}$ Boston Children's Hospital, Boston, MA, ${ }^{5}$ Beth Israel Deaconess Medical Center, Boston, MA

Introduction: A new and novel form of telemedicine is symptom checkers, which are online tools that use computer algorithms to help patients with selfdiagnosis and/or self-triage. Symptom checkers are becoming increasingly popular, with over 100 million uses per year. Despite this, the clinical accuracy of symptom checkers has not been assessed. Our objective was to determine the diagnostic and triage accuracy of online symptom checkers.

Methods: We performed an audit study of Web-based symptom checkers using standardized patient (SP) vignettes to assess their clinical performance. First, we identified 23 symptom checkers that were in English, free, publiclyavailable, and provided advice across a range of conditions. We then compiled 45 standardized patient (SP) vignettes equally divided into three categories of triage urgency: emergent care required (e.g., pulmonary embolism), nonemergent care reasonable (e.g., otitis media), and self-care reasonable (e.g., viral upper respiratory illness). For symptom checkers that provided a diagnosis, our main outcomes were whether the symptom checker listed the correct diagnosis first or within the first 20 potential diagnoses $(n=770$ SP evaluations). For symptom checkers that provided a triage recommendation, our main outcomes were whether the symptom checker recommended emergent care, non-emergent care, or self-care ( $\mathrm{n}=532 \mathrm{SP}$ evaluations).

Results: The 23 symptom checkers provided the correct diagnosis first in $34 \%$ (95\% confidence interval [CI], 31 to 37) of SP evaluations, listed the correct diagnosis within the top 20 diagnoses given in 58\% (95\% CI 55 to 62) of SP evaluations, and provided the appropriate triage advice in 57\% (95\% CI 52 to 61) of SP evaluations. Triage performance varied by con- dition urgency, with appropriate triage advice provided in 80\% (95\% CI 75 to 86) of emergent cases, 55\% (95\% CI 47 to 63) of non-emergent cases, and 33\% (95\% CI 26 to 40$)$ of self-care cases $(p<0.001)$. Performance across the 23 individual symptom checkers on appropriate triage advice ranged from $33 \%$ (95\% CI 19 to 48) to $78 \%$ (95\% CI 64 to 91) of SP evaluations.

Conclusions: Symptom checkers had deficits in both triage and diagnosis. Triage advice from symptom checkers is generally risk-averse, encouraging users to seek care for conditions where self-care is reasonable.

\section{REFERENCE}

1. Fox, S., \& Duggan, M. (2013). Health Online 2013. Washington D.C.: Pew Research Center and California Healthcare Foundation.

\section{Objectives}

1. Utilize a methodology to assess the performance of similar tools to symptom checkers

2. Understand the spread in the clinical performance of publicly available symptom checkers

3. Recognize the role of symptom checkers in comparison to other sources of healthcare information and advice

\section{TUESDAY, MAY 17, 2016}

11:00 AM-12:00 PM

Tuesday, May 17, 2016

\section{Session 49 \\ Session Title: INVESTIGATING THE USE OF STANDARDIZED 2D AND 3D TELEDERMATOLOGY IMAGING IN TRIAGING SKIN CONCERNS}

MODERATOR: Trilokraj Tejasvi, MD, Clinical Lecturer,

Department of Dermatology

University of Michigan Medical School, Ann Arbor, MI

PRESENTER: Thomas Scornavacca, DO, Medical Director, Office of Clinical Integration

UMass Memorial Medical Group, Worcester, MA

PRESENTER: Mary Maloney, MD, Chief of Dermatology

UMass Medical School, Worcester, MA

With the boom in direct-to-consumer teledermatology, many questions have been raised about the accuracy of teledermatology diagnoses and patient compliance in following up with teledermatologist's prescribed treatment plans. While physician-to-physician teledermatology may increase patient compliance, image quality, regulatory, and reimbursement, barriers still prevent its widespread use. The goal of this study was to approach teledermatology as one might a novel radiology test, using in-person dermatologists' responses and biopsy results to compute sensitivity and specificity values for a multi-modality teledermatology imaging system.

Patients with various skin concerns, including pigmented and nonpigmented lesions, rashes, acne, and chronic conditions, had pictures of their skin taken with multi-modality imagers. Collected images included crosspolarized, non-polarized, magnified (dermatoscopic), overview, and 3D modalities. The imaging technology's software portal walked the imaging personnel through a strict protocol, which lasted less than one minute per patient. Each patient was also seen by an in-person (IP) dermatologist. The responses of the IP dermatologists or the biopsy results (when applicable) were used as the "true answer", and served as the study's control group. Most patients were imaged at the dermatology office at time of visit, with a subset of the patients imaged at primary care to more accurately mimic real world conditions. Each 


\section{ORAL PRESENTATIONS ABSTRACTS}

skin concerns' set of images was read by three different teledermatologists (TD), none of which had seen the patient in-person. In line with other concordance studies, the majority TD response was used to compare against the IP dermatologists' response or the biopsy result (when applicable).

The study used a sample size of 350, which was calculated with a minimum tolerable sensitivity of $90 \%$ and a minimum tolerable specificity of 95\%. The primary target was the binary decision to refer or to not refer a patient for an in-person dermatology appointment. Preliminary results show that the imaging system allowed teledermatologists to match in-person dermatologists' triage assessments nearly equivalently, with a non-significant increase in false positives, cases where the teledermatologist requested a biopsy for a lesion that was labeled benign by the in-person dermatologist. The secondary target was the effectiveness of the triage in terms of cost savings. The preliminary results show that this teledermatology protocol could reduce inperson referrals from primary care by $53-75 \%$. Some data collection and data analysis are still ongoing as more teledermatologists' responses are collected and other metrics, such as concordance of primary treatment plans, are investigated. Results will be finalized by the end of this year.

The major takeaway from this study is that by using multi-modality imaging in a standardized protocol at primary care offices, providers can capture the relevant information remote dermatologists need to make safe and accurate triage decisions. The implication for cost savings if this system were to be implemented within the UMass Memorial health network would be twofold in that the number of unnecessary in-person referrals would be reduced while patients with a time sensitive condition, such as melanoma, could have their appointments expedited. This study has led to a rather large and informative image database that lends itself to future collaborations. Follow-up work could include identifying which types of skin concerns have the highest diagnostic accuracy with subsets of imaging modalities and assessing the dermatologist learning curve in remote read times and accuracy rates.

\section{REFERENCES}

1. Barbieri J, Nelson $C_{1}$ James $W$, et al. The reliability of teledermatology to triage inpatient dermatology consultations. JAMA Dermatol 2014; 150(4):419-24.

2. Martin I, Aphivantrakul PP, Chen K, Chen SC. Adherence to teledermatology recommendations by primary healthcare professionals: strategies for improving follow-up on teledermatology recommendations. JAMA Dermatol 2015; 151(10):1130-32.

3. Warshaw EM, Gravely AA, Nelson DB. Reliability of store-and-forward teledermatology for skin neoplasms. J Am Acad Dermatol 2015; 72(3):426-35.

\section{Objectives}

1. Identify key problems facing implementation of physician-to-physician teledermatology today

2. Apply lessons learned from this study when selecting teledermatology technologies and creating local teledermatology networks

3. Devise studies using the model created to calculate teledermatology savings for a health system wanting to implement teledermatology in the future

\section{TUESDAY, MAY 17, 2016}

1:15 PM-2:15 PM

Tuesday, May 17, 2016

\section{Session 57 Session Title: TELEREHABILITATION SUPPORT FOR BRAIN INJURY}

MODERATOR: Tammy Richmond, MS, OTRL, FAOTA, Chief Executive Officer and Founder

Go 2 Care, Los Angeles, CA

\section{USING TELEPRACTICE TO PROVIDE META-COGNITIVE}

SUPPORT FOLLOWING TRAUMATIC BRAIN INJURY:

ONE WOMAN'S EXPERIENCE

PRESENTER: Deidre Sperry, MSc, S-LP(C), Speech Pathologist

and Clinical Director, Paul McCormack, BA

eCommunity Support Network, Hamilton, ON, Canada

Each year hospital Emergency Rooms across North America are inundated with patient visits related to Traumatic Brain Injuries (TBI) including concussion. The Centres for Disease Control and Prevention estimate that in the United States alone there are up to 5.3 million people living with TBI related disabilities. The impact on public health is considerable. Commonly, people living with TBI report difficulties related to attention, fatigue, headaches, difficulties remembering, planning, anticipating, initiating, and problem solving. A TBI can adversely affect a person's ability to independently carry out many functional everyday activities. This population often requires a lifetime of ongoing support to help them navigate the landlines of everyday life. However, for many this help is difficult to find, very expensive, geographically unavailable or not focused on the everyday help they require. In Canada, the story is much the same. Although the incidence of injury is less, the prevalence is just as far reaching with the same societal tolls. In addition, our challenging geographic realities makes rehabilitation and support unattainable for many brain injury survivors.

In 2011, four independent community-based rehabilitation clinicians in Hamilton, Ontario Canada developed a service that would revolutionize the way support is provided to survivors of TBI. By using readily available technology and by delivering services over the Internet many previously encountered obstacles were being torn down. For the last five years brain injury survivors have continued to be served using this model called eRehab. The program offered in Hamilton, Ontario is a social enterprise with a social mission to revolutionize how technology can be used to support people with complex needs. By merging front line knowledge of delivery of quality community based support services and their interest in technology this small program created a truly innovative approach to service.

The subject of this case study is a 54 year old woman who sustained her brain injury in a motor vehicle accident and as a result had many neurocognitive impairments with a myriad of functional sequela. For several months post injury, she participated in a traditional community based, kneeto-knee, multi-disciplinary rehabilitation program, but still she could not participate in many of her everyday life activities such as taking care of her home and family, let alone returning to work. She was depressed and feeling depressed much of the time. Her Occupational Therapist recommended that she participate in the eRehab program. Within a short period of time, the subject of the case study began to experience real life, and demonstrated functional changes. By having the support to implement the meta-cognitive strategies and compensatory strategies on a regular basis without having to leave her home or have someone come to her home, she began to experience greater success and improved quality of life. With the right support, at the right time, delivered in this online manner, the subject began to once again participate in her life roles.

This case study will describe the eRehab program and will demonstrate how the implementation of tele-rehabilitation contributed to the functional and meaningful changes seen in the one woman's life. The presentation will compare models and explore possible reasons why the significant changes were observed when they had not been previously. The presentation will explore some of the challenges experienced in developing and setting up a tele-rehabilitation program such as eRehab, such as colleague adoption and acceptance. Finally, this presentation will discuss how people living with cognitive limitations can live more independent and rewarding lives with the introduction of tele-rehabilitation into traditional models. 


\section{REFERENCES}

1. Ng EM, Polatajko HJ, Marziali E, Hunt A, Dawson DR. Telerehabilitation for addressing executive dysfunction after traumatic brain injury. Brain Inj 2013; 27(5):548-64.

2. Togher L, Wiseman-Hakes C, Douglas J, Stergiou-Kita M, Ponsfrod J, Teasell R, et al. INCOG recommendations for management of cognition following traumatic brain injury, part IV: Cognitive communication. J Head Trauma Rehabil 2014; 29(4):353-68.

3. U.S. Department of Health and Human Services Centre for Disease Control and Prevention (2014). Report to Congress Traumatic Brain Injury in The United States: Epidemiology and Rehabilitation.

\section{Objectives}

1. Identify ways tele-rehabilitation can help people living with TBI

2. Describe challenges and solutions in the provision of tele-rehabilitation

3. Apply lessons learned in this case study to other population groups

MOTOR TELE-REHABILITATION INTERVENTION FOR ACQUIRED BRAIN INJURY: SINGLE-MULTI-USER SERVICE DELIVERY

PRESENTER: Patrice (Tamar) L. Weiss, PhD, Researcher ${ }^{1,2}$, Rachel Kizony, $\mathrm{PhD}^{2,3}$, Sharon Harel, $\mathrm{MSc}^{1}$, Orit Elion, $\mathrm{PhD}^{1}$, Alexei Obuhov, $\mathrm{MD}^{3}$, Gabriel Zeilig, $\mathrm{MD}^{3}$, Yoram Feldman, MBA, BScEng ${ }^{1}$, Mordechai Shani, $\mathrm{MD}^{1}$

${ }^{1}$ Gertner Institute, Ramat Gan, Israel, ${ }^{2}$ University of Haifa, Haifa, Israel,

${ }^{3}$ Sheba Medical Center, Ramat Gan, Israel

Introduction: Tele-rehabilitation technology aims to deliver healthcare services at a distance via synchronous and/or asynchronous techniques. The field has been growing rapidly due to significant advances in technology coupled with an increasing need to provide long-term care to clients, especially those who are remote; insufficient numbers of clinicians available to provide therapy; and the increasing financial burden to healthcare funders (Moffet et al., 2015). For reasons related to client safety, legislative restrictions and technology capability, the majority of tele-rehabilitation services are synchronous, wherein a local therapist provides treatment to a remote client on a one-to-one basis. The Kinect-based ReAbility Online Tele-Motion system was designed to provide a continuum of services ranging from full one-to-one, synchronous care to more efficient interventions in which a single clinician treats two clients simultaneously or in which offline (asynchronous) treatment is supplemented with scheduled synchronous sessions (Kizony et al., 2014). The goal of this service is to improve or maintain the function of the weak upper extremity. The objective of this abstract is to present the feasibility of the service, the clients' clinical profile and results of a retrospective study on clients with Acquired Brain Injury (ABI) enrolled in this service for two months or more.

Methods: Clients with ABI are eligible for this service provided they have intact sitting balance, mild-moderate impairment of the upper extremity, an ability to interact with the system, intact or mild cognitive impairment and accessibility to the Internet at home. They were evaluated prior to the service with the National Institutes of Health Stroke Scale (NIHSS), the MiniMental State Exam (MMSE) or the Montreal Cognitive Assessment (MOCA) for cognitive screening, the Trail Making Tests (TMT, parts A \& B) to assess attention and executive functions, shoulder range of motion, the FuglMeyer Assessment (FMA) to assess active movements of the weak upper extremity and the Motor Activity Log to query how much (MAL amount) and how well (MAL Quality) the person uses his weak hand for daily activities. Initially, clients received one-to-one online treatment twice a week with the level of difficulty for each activity set by the clinician in accordance with their abilities and needs. For clients who are able to do so, treatment is then given by one clinician to two clients at the same time. These clients are also able to perform the activities offline (independently) for up to $45 \mathrm{~min}$ per day on their own. After two months, the client is asked to return for full clinical re-assessment. At this time, a feedback questionnaire (satisfaction from the service) is also administered.

Results: Fifty-eight people (58.6\% male), ranging in age from 10 to 80 years (mean \pm standard deviation $(\mathrm{SD})=56.4 \pm 14.8$ ), were included in the study. The majority of the clients had a stroke (72.4\%), seven (12.1\%) are diagnosed with multiple sclerosis, three had a traumatic brain injury (5.2\%) and the rest had other neurological conditions such as encephalitis. At the start of the tele-rehabilitation service, their mean \pm SD MMSE $=27.5 \pm 2.0(n=26)$ and MOCA $=26.5 \pm 2.8(n=31)$ indicating that most of the clients has intact or mild cognitive impairment. The NIHSS score $=5.4 \pm 1.5$ indicating a mild-moderate level of impairment. The MAL amount $=1.5 \pm 1.0$ and MAL Quality $=1.4 \pm 0.8$, indicating that clients have little use of their weak upper extremity for daily activities. The FMA ranges between 8-54 (35.7 \pm 12.4$)$ and the mean shoulder range of motion (ROM) is greater than 90 degrees (Flexion $109.5 \pm 39.0$; $\mathrm{Ab}$ duction $91.9 \pm 30.4$ ). The mean \pm SD of the TMT shows a large variance (TMT $\mathrm{A}=66.1 \pm 53.5 \mathrm{~s}(\mathrm{n}=28)$ and TMT B $162.7 \pm 124.6 \mathrm{~s}(\mathrm{n}=29))$. Forty-two clients underwent a second assessment after two months (with some missing data due to technical problems or the fact that some tests were not administered at the outset). No adverse events were reported and the clients' feedback of the service (on a scale of $1=$ not at all to $5=$ very much) showed that they enjoyed it $(4.0 \pm 1.1$, median $=4.0)(n=40)$. Initial analysis showed that they had a significant improvement in their FMA score $(\mathrm{t}(37)=-2.5 \mathrm{p}=.017)$, shoulder flexion and abduction ROM $\mathrm{t}(41)=-2.2, \mathrm{p}=.036 ; \mathrm{t}(39)=-2.4, \mathrm{p}=.02$, respectively and TMT B time $(\mathrm{t}(26)=2.4, \mathrm{p}=.027)$.

Conclusions: The results of this small retrospective study demonstrate both the feasibility of a combined synchronous-asynchronous tele-motionrehabilitation service as well as its clinical utility. Subjective feedback was also very good and clients thought it should be combined with conventional rehabilitation services. The service appears to be both effective in important clinical outcomes related to improvements in upper extremity function (e.g., FMA) as well as in attention and executive functions. However, a randomized clinical trial should be conducted to verify this.

\section{REFERENCES}

1. Kizony R, Weiss PL, Elion O, Harel S, Baum-Cohen I, Krasovsky T, Feldman $Y$ Shani, M. Development and validation of a tele-health system for stroke rehabilitation. Int J Disabil Hum Dev 2014; 13(3):361-68.

2. Moffet $H$, Tousignant $M$, Nadeau $S$, Mérette $C$, Boissy $P$, Corriveau $H$, Marquis $F$, Cabana F, Ranger P, Belzile EL, Dimentberg R. In-home telerehabilitation compared with face-to-face rehabilitation after total knee arthroplasty: A noninferiority randomized controlled trial. J Bone Joint Surg Am 2015; 97(14):1129-141.

\section{Objectives}

1. Demonstrate awareness of the relative advantages of synchronous and asynchronous tele-rehabilitation systems

2. Identify the effectiveness and usability of the presented tele-rehabilitation system

3. Become more aware of the methods underlying retrospective clinical studies

TUESDAY, MAY 17, 2016

3:00 PM-4:00 PM

Tuesday, May 17, 2016

\section{Session 65 \\ Session Title: INNOVATIVE APPLICATIONS FOR TELEREHABILITATION}

MODERATOR: Christopher Peterson, Telerehabilitation Program Director Hartford Healthcare Rehabilitation Network, Hartford, CT 


\section{ORAL PRESENTATIONS ABSTRACTS}

\section{LISTENING AND SPOKEN LANGUAGE TRAINING FOR CHILDREN WITH} COCHLEAR IMPLANTATION THROUGH TELEREHABILITATION

PRESENTER: Andi Saptono, PhD, Assistant Professor ${ }^{1}$, Michelle Parfitt, MA, CCC-SLP, LSLS Cert. AVEd ${ }^{2}$, Sujini Ramachandar, MS, CC-SLP ${ }^{1}$, Deborah Moncrieff, Ph. D ${ }^{1}$, Bambang Parmanto, $\mathrm{PhD}^{1}$

${ }^{1}$ University of Pittsburgh, Pittsburgh, PA, ${ }^{2}$ DePaul School for Hearing \& Speech, Pittsburgh, PA

Introduction: Proper Listening and Spoken Language (LSL) training for children with cochlear implantation is necessary to ensure that the recipient's spoken language skill progresses similarly to their typically developing normal hearing peers. Parental involvement and participation has been recognized as a key factor in the success of the rehabilitation. However, as LSL strategies are not intuitive for normal hearing parents, proper education for parents is important to ensure the continuation of therapy in the home setting. The shortage in the number of LSL service providers requires parents and their children to travel to specific sites for training, potentially limiting the amount of training that both the children and parents can receive. Telerehabilitation offers a solution to connect the LSL specialist with their clients: the technology allows the service provider to connect and both educate and train parents to provide intervention for their child in the home setting; when the child is receiving specialist training at school, the same technology provides the opportunity for parents to remotely view and learn from the interaction at school.

Methods: Two families were recruited to the pilot therapy spanning 12 months. After meeting the families in person, researchers conducted assessments to determine the appropriate technology for the project: flexibility (system that allows fine tuning to match with the available bandwidth and technology to deliver appropriate audio and video quality for the therapy) and security (system that has appropriate confidentiality and privacy measures). Prior to a session, the LSL specialist sent an e-mail with lesson plans and education materials. A typical session started with a review of events from any past session and plans to solve any identified problem issues. Sessions mainly consisted of parent-directed activities with the LSL specialist participating only when required. A usability questionnaire measured the perceived effectiveness and satisfaction of telerehabilitation as a platform to deliver the service.

Results: VISYTER was used as the platform for the therapy due to (1) the ability to fine tune the quality of video and audio based on available technology resource and (2) the security of the communication channel. Peripheral equipment such as a speakerphone helped eliminate echo and provide handsfree communication so that parents could communicate with the clinician while interacting freely with their children. A lending library was set-up to provide any peripheral/equipment that the family did not have. Based on an analysis from sound samples and standardized assessments, the children showed progress with their speech and language goals. Feedback from parents indicated that they felt empowered to actively participate in the training and had confidence to continue the interaction and language training for their children themselves. Parents also felt comfortable with telerehabilitation and were able to interact with clinicians without any difficulties or reservations.

Conclusions: Telerehabilitation provides a convenient way to deliver parent education and LSL therapy to children with cochlear implantation. Proper planning and preparation are essential to create a suitable home environment for therapy, including: ensuring conditions that facilitate optimal interaction (ample light, proper position of camera and monitor), minimizing distraction (turning off phone, preparing activities to engage other family members, siblings, and/or friends of children), and keeping the child motivated to stay focused and interested with the therapy (preparing toys, picture books). To mitigate potential technology failures during a session, proper initial setup and backup plans were crucial. During the initial setup, a connection test was conducted prior to the very first session. Educational materials were sent before any session and made accessible via e-mail and/or cloud-based storage.
An alternative communication line such as phone or text messaging were also set up as backup. These strategies were important to ensure a smooth session in times where Internet connectivity was unstable.

\section{REFERENCES}

1. Watkin $P$, et al., Language ability in children with permanent hearing impairment: the influence of early management and family participation. Pediatrics 2007; 120(3):e694-e701.

2. Cason J. A pilot telerehabilitation program: Delivering Early Intervention Services to Rural Families. Int J Telerehabil 2009; 1(1):29-38.

\section{Objectives}

1. Understand the need of speech and language training in home setting 2. Understand challenges as well as the pros and cons of the solutions to deliver the training to the setting

3. Understand the client's impression and experience in using telerehabilitation in a home environment

A PILOT STUDY COMPARING THE EFFICACY OF ASYNCHRONOUS VIDEO BASED TELE-REHABILITATION WITH TRADITIONAL OUT PATIENT PHYSICAL THERAPY FOLLOWING TOTAL KNEE ARTHROPLASTY

PRESENTER: Stefano A. Bini, MD, Professor of Orthopedic Surgery ${ }^{1}$, John Mehajan, MD ${ }^{2}$

${ }^{1}$ University of California San Francisco, San Francisco, CA, ${ }^{2}$ San Francisco Orthopedic Residency Program, San Francisco, CA

Background: A handful of articles have documented successful post-operative tele-rehabilitation following TKR using video based visits. Several problems have been identified ranging from bandwidth and technology issues to minimal improvements in cost of care. Furthermore, little is known about patient outcomes using telemedicine. Asynchronous video platforms promise to address many of these problems but have not been studied.

Introduction: We performed a process improvement pilot study comparing an asynchronous video based software platform to in-person outpatient Physical Therapy (PT) visits following total knee replacement (TKR) while measuring the user experience as well as clinical and patient reported outcomes.

Materials and Methods: Patients undergoing unilateral TKR were recruited into a pilot study at a single institution utilizing standardized TKR care protocols. 51 patients were randomized to one of two study groups. The intervention group (IG) used an application through which the therapist and patient securely exchanged exercise videos and messages asynchronously but were not precluded from accessing outpatient therapy. The control group (CG) received traditional outpatient physical therapy. Baseline data was collected prior to surgery and outcomes, utilization and satisfaction data at 3 months.

Results: 29 patients completed the pilot. The average IG participant logged in 49 times, uploaded 9 videos, 5 photographs and 10 messages. The therapists logged in on average 14 times per patient, for approximately 4.5 ' and spent a total of approximately an hour on line per patient. TC patients reported traveling 84 ' per visit to PT and accessed twice as many group visits. Daily home exercise time was longer for traditional care patients (TC: 71' vs. IG: 59'). Using a Likert scale, both groups found it easy or very easy to contact their therapist (4.5/5.0). The IG reported spending 68' per week on the application and rated the overall experience as 4.2 out of 5. On the VAS, the average improvement in pain at 3 months was equivalent for both groups (TC: 4.0, IG: 3.8) as was the final VAS score (TC: 1.5, IG: 1.7). The KOOS score improvement was equivalent between the two groups (TC: 7.2, IG: 7.9) as was the overall final score (TC: 5.2, IG: 4.9).

Discussion: In this pilot study we report quality outcomes following asynchronous telerehabilitation that are generally not inferior to those achieved with a traditional in person care model despite much lower utilization of costly outpatient resources. Further, patient and provider satisfaction was high, as was patient utilization of the application. 


\section{ORAL PRESENTATIONS ABSTRACTS}

Conclusion: The results of this pilot study suggest that the use of asynchronous telerehabilitation is a viable alternative to both synchronous video visits and the in-person care model. Patient satisfaction was high and clinical outcomes were equivalent between groups. Resource utilization was lower in the intervention group. The results of this pilot study suggest that asynchronous telerehabilitation following TKA can deliver excellent quality and lower cost over existing models of care in selected patients and justifies further study.

\section{REFERENCES}

1. Moffet $H_{\text {, Tousignant } M}$, Nadeau $S$, Mérette $C$, Boissy $P$, Corriveau $H$, et al. InHome Telerehabilitation Compared with Face-to-Face Rehabilitation After Total Knee Arthroplasty. J Bone Joint Surg Am 2015; 97(14):1129-141.

2. Tousignant M, Boissy P, Moffet $H$, Corriveau H, Cabana F, Marquis F, Simard J. Patients' satisfaction of healthcare services and perception with in-home telerehabilitation and physiotherapists' satisfaction toward technology for post-knee arthroplasty: an embedded study in a randomized trial. Telemed J E Health 2011; 17(5):376-82.

3. Tousignant $M$, Boissy $P$, Corriveau $H$, Moffet $H$. In home telerehabilitation for older adults after discharge from an acute hospital or rehabilitation unit: A proof-of-concept study and costs estimation. Disabil Rehabil Assist Technol 2006; 1(4):209-16.

\section{Objectives}

1. Understand the different value propositions between synchronous and asynchronous telemedicine platformse

2. Discuss the impact on patient care of providing home based post operative rehabilitation

3. Evaluate how telerehabilitation will help us achieve the IHI Triple Aim

\section{TUESDAY, MAY 17, 2016}

4:15 PM-5:15 PM Tuesday, May 17, 2016

\section{Session 73 Session Title: PRENATAL CARE AND TELEMEDICINE}

MODERATOR: Prashant G. Deshpande MD, FAAP, Pediatrician Advocate Health Care, Burr Ridge, IL

SELF-ADMINISTERED NON-STRESS TESTING: REMOTE FETAL MONITORING IN A HIGH-RISK OBSTETRIC POPULATION

PRESENTER: Erin Clark, MD, Assistant Professor

University of Utah, Salt Lake City, UT

Introduction: Mobile connectivity can potentially impact medical care of many patient populations, including pregnant patients. Non-stress testing (NST) that relies on fetal tococardiography technology is a widely accepted method of identifying fetal compromise that may result in stillbirth. NSTs are routinely used for high-risk pregnancies in which there is a higher risk of stillbirth secondary to maternal, fetal, or pregnancy-related complications. NSTs are generally performed in an in-hospital or outpatient clinical setting. NSTs require a bed, mid-level provider, interpreting physician, and NST machine. Most facilities are limited in the number of NSTs that can be performed at any given time secondary to NST machine and spatial limitations. Studies have examined conducting remote NSTs using telephone-photograph pairing; the earliest studies were performed in the late 1970s. The results of these studies encouragingly indicated that interpretable NST monitoring strips could be obtained in remote locations and transferred to a central location for reading. In addition to feasibility assessments, costeffectiveness studies of remote fetal monitoring systems from the late 1980s to the early 2000s showed decreased costs for at-home fetal monitoring, even when a midlevel provider conducts the in-home testing. While most of these studies looked at provider-applied devices, some research has shown patients can be taught how to use an NST device reliably, performing the monitoring to a similar competency as midlevel providers. Studies of patient satisfaction for fetal in-home monitoring indicate that remote testing is acceptable to patients and associated with a high level of satisfaction. It is important to note that no studies in the last decade have validated or evaluated the utility of modern at-home, patient-applied, remote NST monitoring systems, nor have any studies looked at the steps required to integrate at-home NST monitoring into an existing medical system.

Methods: Healthcare systems, information technology and mobile health (m-Health) platforms have all evolved, with newer and more efficient systems of electronic fetal monitoring that allow for remote fetal monitoring of highrisk pregnancies. For this project, patients with physician-ordered twiceweekly NSTs scheduled in the OB Diagnostic Center at University of Utah Hospital were eligible for enrollment. Patients living greater than 60 miles from the healthcare center were excluded. Study participants were asked to use the remote NST system machine every week until the time of delivery, while also receiving an in-clinic NST weekly. Participants were trained how to use the remote NST device and demonstrated competency prior to study enrollment. Patients were given educational materials, including a visualsenhanced quick-start guide. For their remote NST monitoring, patients received a virtual appointment. At the scheduled time, the patient applied the monitor, and the NST was read by the mid-level provider staffing the testing center after a 30-minute strip was recorded and transmitted to the database. Patients were notified of the result using a HIPPA-compliant telemedicine platform, and appropriate follow-up was arranged according to the NST management algorithm developed for this study. NSTs were also interpreted by a maternal-fetal medicine physician, according to the established clinical algorithm. Each patient served as her own control in terms of NST time, NST interpretability, and satisfaction. The weekly on-site NSTs provided educational opportunities and facilitated trouble-shooting. Study participation continued until the provider discontinued the NST order or until delivery.

Results: The primary outcome being measured is the feasibility and steps required to integrate remote NST monitoring into an existing healthcare system:

- Scheduling

- Interpretation and communication

- Clinical management algorithm

- Medical billing

- Secondary outcomes:

- Percent of interpretable NSTs obtained

- Time required by staff and patients to obtain an adequate NST tracing

- Frequency of necessary and appropriate follow-up according to the clinical algorithm

- Frequency/type of complications identified

- Patient/provider satisfaction

- Cost-effectiveness for the patient and healthcare system

Conclusions: The aim of this study is to evaluate the feasibility of integrating a remote self-administered NST program into an established healthcare system, to determine patient and healthcare provider satisfaction, and to determine costeffectiveness for the healthcare system and patient. The research results are anticipated in early 2016. Healthcare systems are becoming increasingly interested in implementing innovative care strategies in order to improve the quality of healthcare, reduce cost, and improve patient satisfaction- in other words, create VALUE. Within obstetrics, remote NSTs show promise for helping to achieve these aims. Our research will consider whether this technology could develop into a viable fetal testing option for healthcare systems across the nation.

\section{Objectives}

1. Demonstrate successful integration of a remote self-administered NST monitoring program into an established healthcare system 


\section{ORAL PRESENTATIONS ABSTRACTS}

2. Assess patient and healthcare provider satisfaction with a remote NST program

3. Evaluate cost-effectiveness of a remote NST program for the healthcare system and patient

\section{REMOTE PRENATAL CARE FOR LOW-RISK PREGNANT WOMEN}

PRESENTER: Brandon Welch, MS, PhD, Assistant Professor

Medical University of South Carolina, Charleston, SC

Introduction: The American College of Obstetricians and Gynecologists (ACOG) recommends that women receive roughly 12-15 prenatal care visits during pregnancy. While this level of care may be necessary for some women, it may be more than necessary for others; particularly women at low-risk for pregnancy complications who have had one or more successful uncomplicated pregnancies. With recent reimbursement changes toward bundled payments for prenatal care and delivery, the frequency of prenatal care visits for low-risk women may represent unnecessary expenses to a healthcare organization. As a result, clinicians and researchers at the University of Utah developed a new remote prenatal care protocol for low-risk pregnancies involving telemedicine. Pregnant women in this remote care protocol receive five in-person prenatal care visits at key times during the pregnancy (weeks 12, 20, 28, 36, 40+) with the remaining visits (weeks 16, $24,30,32,34,37,38,39)$ provided remotely using telemedicine technology. To support remote care, pregnant women were provided with at-home fetal heart monitors, digital blood pressure, and weight to obtain vital measurements at home. They then reported their results before each remote appointment using patient-entered flowsheets within Epic MyChart. At the scheduled appointment time, patients would meet with their prenatal care provider using a HIPAA-compliant, Web-based telehealth video solution (Doxy.me). By reducing costs and providing a more convenient option for patients, this telemedicine approach has the potential to greatly change the prenatal care paradigm in the United States. The objective of this research study was to compare how the remote prenatal care strategy compares to standard prenatal care.

Methods: To systematically evaluate how remote prenatal care compared to standard prenatal care, we conducted a randomized control trial with 200 lowrisk women, 100 participants were assigned to each arm (remote care vs usual care). Using REDCap, surveys were distributed to participants at pre-determined times throughout the pregnancy. The primary outcome of the study compared patient satisfaction in the remote prenatal care arm to standard inperson prenatal care. Satisfaction surveys were distributed at weeks 16, 24, and 34. We also developed a model to estimate patient cost and burden for remote and in-person methods of care for comparison. These cost assessments occurred in weeks $20,24,28,34,36$. Within the remote care arm, we ascertained user experiences using the remote care technology, and their preference for remote prenatal care. To validate the at-home vitals measurements, participants in the remote care arm recorded their measurements prior to having the measurements collected in the clinic during in-person appointments (weeks 20, 28, 36).

Results: From August 2013 to August 2015, we successfully enrolled all 200 low-risk pregnant women to participate. As of this writing, the last set of participants are completing the protocol. Data collection will be complete with the final delivery around January 2016, at which time formal results analysis will commence and be reported. Nevertheless, participant feedback and preliminary results thus far indicates strong evidence that telemedicine is an acceptable and cost effective approach to prenatal care for low-risk women.

Conclusions: By demonstrating that remote prenatal care is an acceptable and cost effective way to deliver prenatal care to low-risk women, it could revolutionize how prenatal care is delivered in the United States and abroad. This study provides evidence to support the new remote prenatal care paradigm.

\section{REFERENCE}

1. Guidelines for perinatal care, 7, American Academy of Pediatrics and the American College of Obstetricians and Gynecologists. (Ed), 2012.

\section{Objectives}

1. Understand the benefits of telemedicine in the prenatal care population

2. Learn how telemedicine impacts pregnant moms compared to standard prenatal care

3. Learn how this telemedicine approach was implemented in a practical and scalable way

OBSTETRIC TELE-ULTRASOUND IN THE NGABE-BUGLE REGION OF PANAMA TO IMPROVE MATERNAL AND FETAL OUTCOMES

PRESENTER: Brendan H. Grubbs, MD, Assistant Professor of Obstetrics and Gynecology ${ }^{1}$, Stuart Siegel, $\mathrm{MD}^{2}$, Ryan Navarro, $\mathrm{MHA}^{2}$, Silvio Vega, $\mathrm{MD}^{2}$, Jocelyn Navarro, $\mathrm{BS}^{2}$, Desiderio Castillo, $\mathrm{MD}^{3}$, Yaiset Joseph, $\mathrm{MD}^{3}$, Mariela Castrellon, $\mathrm{MD}^{4}$

${ }^{1}$ University of Southern California/ Children's Hospital Los Angeles, Los Angeles, CA, ${ }^{2}$ Children's Hospital Los Angeles, Los Angeles, CA, ${ }^{3}$ University of Southern California/ Children's Hospital Los Angeles/Comarca Ngabe-Bugle Ministry of Health, Los Angeles, CA, ${ }^{4}$ University of Southern California/ Children's Hospital Los Angeles/Hospital Obaldia, Los Angeles, CA

Introduction: The Ngabe-Bugle region of Panama is home to approximately $90 \%$ of the indigenous population of the country, and has relatively fewer health resources as compared to the surrounding areas. In 2012, the maternal mortality rate was $274.3 / 100,000$, as compared to the rate of $34.3 / 100,000$ seen in the neighboring state of Chiriqui which features more widely available healthcare. Similarly, the infant mortality rates in the indigenous population of Panama is elevated (84/ 1,000 Live Births) when compared to all of Panama (17.2/1,000 Live Births). As part of its goal of improving maternal and child health in developing parts of the world, the Lopez Family Foundation, in conjunction with Children's Hospital Los Angeles and the Panama Ministry of Health, has developed a Prenatal Ultrasound Screening Program to serve the area. Under this program, local General Practice Physicians employed by the Panama Ministry of Health have been trained by academic Obstetricians at Hospital Jose Domingo de Obaldia in Chiriqui to perform obstetric ultrasound assessments. These ultrasounds are routinely performed on patients receiving prenatal care at four of the rural clinics located in Ngabe-Bugle. Potential maternal benefits anticipated from this project include diagnosis of early treatable pregnancy complications including ectopic pregnancy and gestational trophoblastic disease, as well as late complications such as placenta previa. Potential fetal and neonatal benefits include: a more accurate assessment of gestational age, insurance of adequate fetal growth, and diagnosis of birth defects in which fetal rather than neonatal transport to a tertiary hospital would allow for prompt management.

Methods: This retrospective Cohort study will examine pregnancy outcomes in all patients receiving care at 4 Corregimiento (Township) based hospitals (Cerro Iglesias, Hato Juli, Hato Chami, and Sol'oy) as well as Hospital San Felix, which serves the entire Ngabe-Bugle region. Three ultrasound examinations are standardly scheduled for each patient in the First Trimester (10-12 weeks), Second Trimester (20-24 weeks), and the Third Trimester (32-36 weeks). All examinations are performed using a GE Voluson i. The First Trimester Ultrasound is used to determine pregnancy location, number of fetuses, and gestational age. The Second and Third Trimester Examinations are used to screen for fetal anomalies, and to evaluate growth, amniotic fluid. All examinations are recorded electronically, following a set protocol, and the generated images are transmitted via satellite to Hospital Jose Domingo de Obaldia for review by Obstetric staff. Ultrasound data as well as pregnancy outcomes are compiled by the Hospital Obaldia staff on an ongoing basis, and the results of those pregnancies served by the ultrasound program will be 


\section{ORAL PRESENTATIONS ABSTRACTS}

compared to those areas of Ngabe-Bugle not served by the program, as well as to historic controls.

Results: During the study period to date (12/2014-8/2015) a total of 556 ultrasounds have been performed in 398 pregnancies. Of the pregnancies screened to date, 3 congenital anomalies have been diagnosed, which is consistent with an expected anomaly rate of approximately $1 \%$. Overall, the delivery plan was altered by ultrasound findings in 10.5\% of the cases screened.

Conclusions: Our preliminary data from this study supports the feasibility of ultrasound screening by trained General physicians, when they are able to be backed by Obstetricians trained in fetal ultrasound. In the setting of NgabeBugle, a remote region of Panama, this is possible due to the introduction of a portable ultrasound unit and the development of an adequate telemedicine network for the efficient transmission of ultrasound images to Hospital Obaldia. We anticipate that the detection rate of fetal anomalies and prediction of obstetric complications will improve as the newly trained physicians gain ultrasound experience, and that this will translate into improved maternal and fetal outcomes.

\section{Objectives}

1. Understand the potential benefits of implementing an Obstetric Ultrasound Screening Program

2. Understand how telemedicine allows for implementation of an Obstetric Ultrasound Screening Program in a region without previously trained providers

3. Understand how this telemedicine program has affected maternal and fetal outcomes

\section{Track: Mental Health Room 205 AB}

\section{SUNDAY, MAY 15, 2016}

12:00 PM-1:00 PM

Sunday, May 15, 2016

\section{Session 5 \\ Session Title: LEVERAGING TECHNOLOGY TO ENHANCE ACCESS TO SPECIALTY CARE AND FOSTER INTEGRATION AND CARE COORDINATION}

MODERATOR: Ricardo Mendoza, MD, Chief Mental Health Psychiatrist Los Angeles County Department of Mental Health, Los Angeles, CA

PRESENTER: Christopher Benitez, MD, Coordinator for Behavioral Health Special Projects

Los Angeles County Department of Health Services, Los Angeles, CA

PRESENTER: Ricardo Mendoza, MD, Chief Mental Health Psychiatrist Los Angeles County Department of Mental Health, Los Angeles, CA

For too long patients have endured long waits as they exit the primary care door and queue up to see a specialist. Moreover, some referrals for specialty care are inappropriate while others should be fast tracked. Referrals from Primary Care (PC) to Mental Health can be especially problematic.

EConsult is a Web-based, encrypted, HIPAA compliant software platform that is being utilized in Los Angeles County (LAC) to enhance communication between primary care providers and medical specialists. PCPs logon to the software application, attach important elements of the patient's medical record (diagnoses, medications, laboratory values, etc.) and then frame the consultation question. The specialty reviewer (SR) strives to provide meaningful feedback to the PCP within four business days. Based on the data provided by the PCP and the nature of the consultation, the specialist can: 1) engage the PCP in cross talk dialog to better assist him/her in managing the patient, 2) authorize an immediate follow-up appointment, 3) direct the PCP to consult another, more appropriate specialty or, 4) deny the request for a face-to-face specialty care visit.

The Department of Health Services (DHS) and the Department of Mental Health (DMH) are separate and distinct organizational entities in LAC and each possesses a unique Electronic Medical Record (EMR) system. While this separateness has proved advantageous for the seriously and persistently mentally ill (protected funding streams for recovery, community based service delivery), it has also brought about significant challenges for patient movement across both systems and in the coordination of care between providers. The vast majority of patients in the PC setting with comorbid mental health and medical problems suffer with mild to moderate anxiety and depression and do not qualify for specialty mental health services. Stigma also remains an important factor that often prevents patients in the medical setting from seeking mental health treatment. These patients often prefer and need to be treated in their Patient Centered Medical Home. In preparation for the rollout of Psychiatric eConsult in LAC DHS, a supportive management approach was adopted for the web portal home page. An emphasis was placed on educating and assisting the PCP in the proper management of their comorbidly-affected patients. And for that subset of patients that did warrant specialty mental health services in the LAC DMH, workflows to ensure a smooth referral process had to be crafted. In addition, engineering and informatics specialists from each EMR vendor were brought in early on in the process to develop user friendly pathways for health information exchange.

Since its inception in 2012, 53 medical service web portals have rolled out on the eConsult platform in the LAC DHS. Currently, approximately 13,000 eConsults are generated per month. The number of referrals for face-to-face specialty care has been reduced by $30 \%$ and virtually all referrals to specialty care are handled through the eConsult platform. The response time of the SRs is 2.9 calendar days. The average length of time it takes a SR to answer an eConsult is less than eight minutes and the number of cross talks between the PCP and SR is 2-3. In LAC the use of the eConsult platform has evolved over time and depending on the medical service its application can resemble a simple eReferral solution or a collaborative, supportive management tool In light of the outlined challenges, Psychiatric eConsult is one of the last medical services to go live in the system. Data will be collected and compared to the large data set emerging with the use of eConsult in the other medical services.

EConsult represents one tool in the effort to achieve the Triple Aim of Health Care Reform. Based on the LAC experience, technology, in the form of the eConsult platform and HIE solutions, can produce efficient, enhanced communication between PCPs and medical specialists. It can also promote care coordination between providers from disparate systems of care. These results can potentially lead to improved population health and decreased costs. While technology can be utilized across large systems of care to foster integration, careful consideration must be given to the potential operational, technical, legal, organizational and financial barriers to care-coordination, collaboration and integration. When planning such an effort, healthcare administrators must also consider the nature of the clinical and cost outcome measures that will be monitored to determine the effectiveness of eConsult at improving population health.

\section{REFERENCES}

1. Keely $E$ et al. Utilization, Benefits, and Impact of an e-Consultation Service Across Diverse Specialties and Primary Care Providers Telemed J E Health 2013; 19(10):733-38.

2. Liddy $\mathrm{C}$ et al. Ten Steps to Establishing an e-Consultation Service to Improve Access to Specialist Care. Telemed J E Health 2013; 19(12):982-90.

3. Yeuen K et al. Not Perfect, but Better: Primary Care Providers' Experiences with Electronic Referrals in a Safety Net Health System. J Gen Intern Med 2009; 24(5):614-19.

\section{Objectives}

1. Participants will be able to list the types of outcome metrics that should be measured when utilizing the eConsult platform 
2. Participants will be able to list the critical elements that should be included when designing an eConsult web portal home page

3. Participants will be able to describe the types of Health Information Exchange solutions that can be utilized to transmit Protected Health Information across large systems of care utilizing separate electronic medical records

\section{SUNDAY, MAY 15, 2016}

\section{1:15 PM-2:15 PM}

Sunday, May 15, 2016

\section{Session 13 Session Title: A CASE STUDY OF TELEHEALTH BEHAVIORAL CRISIS ASSESSMENTS}

MODERATOR: John Simon, MD, Medical Director Behavioral Healthcare Providers, Golden Valley, MN

PRESENTER: Nicole Bauer, MA, DEC, Telehealth Services Manager Behavioral Healthcare Providers, Golden Valley, MN

PRESENTER: Sally Lewis Olson, MEd, LADC, Director of Marketing and Network Services

Behavioral Healthcare Providers, Golden Valley, MN

Rural emergency departments (ED's) face a continuing challenge of increasing behavioral crises with limited resources to properly assess and treat these patients. Fairview Range Regional Medical Center (FRRMC) in Hibbing, Minnesota serves both rural and frontier communities, with a catchment area over 6,000 square miles. Most behavioral patients assessed in the FRRMC emergency department were admitted on-site for inpatient psychiatric care, jailed or sent many miles away for a psychiatric admission. Inappropriate admissions were taxing the hospital's mental health resources, preventing patients who truly need this level of care from receiving treatment. Additionally, information shared between providers was minimal, often requiring reassessment and prolonged admissions. FRRMC sought a cost-effective, efficient solution that would increase access to resources and strengthen partnerships in their area, with the goal of improving treatment outcomes and patient satisfaction.

In June of 2014, FRRMC began utilizing the DEC services to enhance the treatment of patients presenting with a behavioral health crisis. In 2002, Behavioral Healthcare Providers (BHP), in a partnership with the University of Minnesota Medical Center (UMMC), created an innovative care model called the Diagnostic Evaluation Center (DEC $\left.{ }^{8}\right)$ that provides access in emergent settings to a licensed mental health provider who can thoroughly assess behavioral patients, coordinate appropriate care and follow up with discharged patients. The typical work flow involves patient triage, registration, contact with a clinician, assessment, and referral.

The convergence of multiple technologies allows for an adaptive process that integrates with a variety of care settings. Telehealth technology connects patients and providers in a secure manner, compliant with Health Insurance Portability and Accountability Act (HIPAA) and Health Information Technology for Economic and Clinical Health (HITECH) Act regulations. Additionally, the DEC application safely stores and shares important clinical information with all members of the patient's treatment team. Secure, Webbased scheduling technology allows patients to be connected to appropriate care providers at any time and all follow up information is tracked for reporting and continuity of care purposes.

Creative use of resources has enhanced the delivery of care in rural health settings. On-demand services can be difficult to staff due to unpredictable volumes and assessment times. Leveraging under-utilized clinicians already working on-site in other locations has provided backup support when primary telehealth resources were busy. Average wait times for services delivered in Northern Minnesota are under 30 minutes while assessment duration ranges from 30-45 minutes.
Partnerships and collaborations are critical to the success of a telehealth service. A commitment to networking must be made to improve access to community-based resources for patients needing care. Even when care teams understand what treatment is needed, rural communities struggle to engage the appropriate services. There is typically a huge shortage of outpatient resources available in remote communities and when appointments are made there is no follow up to measure success. A large network of providers is needed to ensure that patient needs are met in a variety of specialties.

The implementation of a telehealth behavioral crisis assessment service in FRRMC has already had a noticeable impact. Of 364 patients assessed at FRRMC, 183 (50.27\%) patients were discharged back into their communities for follow up care, resulting in a reduction of admissions to inpatient care of about 30\% according to hospital leadership. This reduction in inappropriate admissions allowed for better access to inpatient treatment for other community members needing this level of care. Additionally, the improvement in clinical documentation and collaboration among care team members, including DEC clinicians, contributed to reduced lengths of stay at FRRMC, dropping from 9.4 days to 9.0 days on average. Readmission rates to FRRMC's inpatient unit also dropped from $7.7 \%$ to $5.0 \%$, partially accredited to the referral of only clinically appropriate patients for this level of care. Attendance is tracked and over $85 \%$ of patients have attended their scheduled follow up appointments. Improved reporting capabilities have also helped to identify areas of need within the FRRMC care system, providing support to the leadership team who is attempting to expand services.

DEC has shown the ability to create a Web-based tool that shares information, with appropriate releases in place, and has created relationships in the community to improve the workflow with these patients as a viable solution to the ongoing behavioral health crisis we are facing.

Current metrics determined by on-going care delivery.

Objectives

1. Describe several ways in which the use of technology can enhance the delivery of crisis behavioral services in rural settings

2. Facilitate a discussion on networking and partnerships required to successfully treat behavioral crises in rural settings

3. Present metrics that demonstrate the success of delivering telehealth behavioral crisis assessments in rural Minnesota

\section{MONDAY, MAY 16, 2016}

8:00 AM-8:45 AM Monday, May 16, 2016

\section{Session 21 \\ Session Title: BUILDING SUSTAINABLE TELEPSYCHIATRY PROGRAMS: A HOW-TO GUIDE}

PRESENTER: Samir Malik, Chief Executive Officer 1DocWay, New York, NY

Telepsychiatry can be a profit center for all participants-patients, hospitals, clinics, and providers; however, to do so requires building a system that has the right incentives directed at the right players. This tutorial will teach attendees how to build such systems themselves.

For years, telepsychiatry has been dependent upon grant funding, foundation support or charity care funding in order to survive. However, the past few years have seen increasing reimbursement for telepsychiatry, both by government and commercial payors. In addition, by bridging the market between underserved patients and under-utilized hospital beds, telepsychiatry can be used to create competitive advantages for hospitals willing to participate. The environment has been primed for telepsychiatry to take off.

The right business model now exists to promote the sustainable use of telepsychiatry to improve access and outcomes. These models are driven by two key economic factors: firstly, delivering telepsychiatry can be reimbursed, 


\section{ORAL PRESENTATIONS ABSTRACTS}

helping cover the costs of delivering care into underserved communities; secondly, providing telepsychiatry can deliver considerable downstream value to providers in both fee-for-service and at-risk payment models. FFS providers can benefit from reaching new patients and tapping new markets for ancillary services (procedures, inpatient, etc.). At-risk providers benefit from the considerable clinical benefits demonstrated through telepsychiatry, including reduced ED usage and inpatient hospitalizations by patients who have access to telepsychiatry. We will walk attendees through the dynamics involved in each.

Following the above methods, we have importantly seen a level of stickiness and patient engagement that demonstrates the model's sustainability. $85 \%$ of the 20,000 patients we have treated come back for follow up visits. Clinics renew monthly contracts at a 98\% rate, and increase provider utilization 50\% year over year. Our proposed model has treated tens of thousands of patients in 8 states and delivered considerable ROI to its users. Building a hub-and-spoke model of telepsychiatry that focuses on reimbursement has created over $\$ 1,400,000$ in new revenue, at an ROI over 233\% for the hub hospitals delivering these services.

Given the opportunity to treat new patients (83\% of our patients were not seeking any services prior to telepsychiatry), create new revenue, and expand footprint, provider systems now have the opportunity do well by doing good through telepsychiatry. Telepsychiatry no longer has to be a subsidized or charitable endeavor.

\section{Objectives}

1. Teach attendees how to build their own sustainable telepsychiatry programs

2. Highlight strategies to address key challenges such as reimbursement, credentialing and provider selection

3. Build confidence amongst attendees that they can set these programs up effectively and rapidly

\section{MONDAY, MAY 16, 2016}

11:00 AM-12:00 PM

Monday, May 16, 2016

\section{Session 29 Session Title: TELEMENTAL HEALTH TREATMENTS FOR CHILDREN}

MODERATOR: Samir Malik, Chief Executive Officer 1DocWay, New York, NY

THE CHILDREN'S ADHD TELEMENTAL HEALTH TREATMENT STUDY (CATTS): REMOTE TRAINING OF THERAPISTS IN EVIDENCE-BASED CAREGIVER TRAINING AND CAREGIVERS' OUTCOMES

PRESENTER: Kathleen M. Myers, MD, MPH, MS, Professor, Director of Telemental Health ${ }^{1}$, Ann Vander Stoep, $\mathrm{PhD}^{2}$

${ }^{1}$ University of Washington and Seattle Children's Hospital, Seattle, WA

${ }^{2}$ University of Washington School of Medicine and School of Public Health, Seattle, WA

Introduction: The past decade has seen increased use of telehealth (TH) technologies to support primary care providers' (PCPs) in underserved geographic areas in their care of children with mental health conditions. ${ }^{1}$ The empirical evidence supporting TMH effectiveness with children and families is building incrementally. Studies have demonstrated the feasibility of providing TMH services to youth. ${ }^{2} \mathrm{PCPs}$ and caregivers have endorsed high levels of satisfaction with these services. ${ }^{3} \mathrm{~A}$ few small studies have tested TMH effectiveness in improving mental health outcomes for children. The Children's ADHD Telemental Health Treatment Study (CATTS) was a randomized controlled trial that compared the effectiveness of a new TMH-mediated service-delivery model to a standard model of telepsychiatry consultation within the context of primary care. Recently we reported results of the CATTS trial that demonstrated the effectiveness of using $\mathrm{TH}$ technologies to improve the care and outcomes for children diagnosed with ADHD is seven underserved communities. ${ }^{4}$ Here we present results regarding caregivers' response to the CATTS service delivery model using a combination of synchronous and asynchronous telehealth technologies to train community therapists in an evidence-based caregiver training intervention. We tested two hypotheses: H1: Caregivers of children assigned to the CATTS model will experience greater reductions in distress compared to caregivers of children randomized to the active control condition. H2: A significant part of the effects of the CATTS model on caregiver distress will be explained by improvement in children's ADHD symptoms.

Methods: We recruited children from primary care providers (PCPs) in seven underserved non-metropolitan communities in Washington and Oregon. Eighty-eight PCPs referred 223 children (ages 5.5 - 12.9 years) to the CATTS trial. Children randomized to the experimental service-delivery arm received six sessions of telepsychiatry paired with six behavior management training sessions for caregivers. The behavior management was delivered in person by community therapists who were trained and supervised remotely using a combination of synchronous and asynchronous technologies. Children randomized to the active control arm received usual primary care (PC) services augmented with one telepsychiatry consultation. Caregiver outcomes included changes in caregiver distress measured by the Patient Health Questionnaire (PHQ-9), Parenting Stress Index (PSI), Caregiver Strain Questionnaire (CSQ) and Family Empowerment Scale (FES). Child outcomes included reductions in ADHD symptoms and improvements in performance (Vanderbilt ADHD Rating System). Caregivers completed five assessments. Multilevel mixed effects regression modeling tested for differences between conditions in caregiver outcomes from baseline to 25 weeks. To test for mediation, we used a Barron \& Kenny framework to establish that the mediator was associated with caregiver outcomes and that the CATTS intervention condition was associated with an improvement in caregivers' outcomes. Previous analyses demonstrated that the CATTS model was associated with greater improvement in child ADHD symptoms. ${ }^{5}$ We used bootstrap estimates of the mediated effects and their confidence limits to determine if effects of the CATTS service delivery model on caregivers' outcomes were significantly mediated through improvement in children's ADHD symptoms.

Results: Compared to caregivers of children in the TMH-augmented usual PC services arm, caregivers of children in the experimental TMH arm showed statistically significantly greater improvements onPHQ-9 $((\beta=-1.41,95 \% \mathrm{CI}=-2.74$, $-.08, \mathrm{p}<.05)$, PSI $(\beta=-10.16,95 \% \mathrm{CI}=15.18,-5.62, \mathrm{p}<.001)$, CGSQ $(\beta=-5.41$, $95 \% \mathrm{CI}=-8.58,-2.24, \mathrm{p}<.001)$ and FES $(\beta=6.69,95 \% \mathrm{CI}=2.32,11.06, \mathrm{p}<.01)$. Improvements in parenting stress, caregiver strain, and family empowerment were partially mediated by improvements in children's ADHD symptoms. In post-hoc analyses, reductions in child inattention, hyperactivity, and ODD each demonstrated significant mediational effects on improvements in caregiver outcomes, with the highest percent of effect explained by child inattention symptoms.

Conclusions: The CATTS trial supports the effectiveness of a short term TMH-mediated service model compared to TMH-augmented PC services as usual in reducing the distress of caregivers of children with ADHD. The intervention was successfully and efficiently delivered by local therapists who were trained remotely and remained an enduring resource for their communities. This evidence will contribute to conversations among stakeholders from advocacy organizations and primary care, mental health, and health policy sectors as they work together to address the needs of families living in communities with low access to child mental health specialty treatment resources. Telehealth technologies can help to train communities in evidencebased care and improve outcomes for distressed caregivers of children with psychiatric disorders.

1. American Academy of Pediatrics. Clinical practice guideline: treatment of the school-aged child with attention-deficit/hyperactivity disorder. Pediatrics 2001; 108(4):1033-44 
2. Lau ME, Way BB, Fremont WP. Assessment of Suny Upstate Medical University's child telepsychiatry consultation program. Int J Psychiatry Med 2011; 42(1): 93-104.

3. Xie Y, Dixon JF, Yee OM, et al. A study on the effectiveness of videoconferencing in teaching parent training skills to parents of children with ADHD. Telemed J $E$ Health 2013; 19(3):192-99.

4. Myers K, Vander Stoep A, Zhou C, McCarty C, Katon W. Effectiveness of a telehealth service delivery model for treating attention-deficit/hyperactivity disorder: a community-based randomized controlled trial. A community-based trial. J Am Acad Child Adolesc Psychiatry 2015; 54(4):263-74.

\section{Objectives}

1. Attendees will learn about using synchronous and asynchronous telehealth technologies to train community therapists in evidence-based interventions

2. Attendees will learn about caregivers' outcomes in response to evidence-based interventions provided in person by therapists trained remotely through telehealth technologies

3. Attendees will learn about the delivery of evidence-based caregiver behavioral training through videoconferencing

DEVELOPMENT AND EVALUATION OF GAMIFIED MOBILE HEALTH SYSTEM TO INCREASE PATIENT MOTIVATION IN CHILD ANXIETY TREATMENT

PRESENTER: Gede Pramana, MS, School of Health and Rehabilitation Sciences ${ }^{1}$, Bambang Parmanto, $\mathrm{PhD}^{1}$, Jennifer S. Silk, $\mathrm{PhD}^{1}$,

Philip C. Kendall, $\mathrm{PhD}^{2}$

${ }^{1}$ University of Pittsburgh, Pittsburgh, PA, ${ }^{2}$ Temple University, Philadelphia, PA

Introduction: Cognitive Behavioral Therapy (CBT) is an efficacious treatment for child anxiety disorders. Although efficacious, many youth (40-50\%) do not show a significant reduction in symptoms and/or full recovery from primary anxiety diagnoses. Youth may fail to improve because they do not apply the skills learned in the clinic to their daily life. Homework is routinely assigned in CBT with the goal of generalizing skills beyond the clinic. However, clinicians have noted that youth are particularly likely to struggle with homework completion. This can occur for a variety of reasons, including (1) not remembering to complete the homework, (2) not wanting to complete the homework, and (3) not understanding the skills needed to complete the homework. Gamified mobile health (m-Health) application provides potential solution to improve CBT efficacy by delivering more playful and interactive Ecological Momentary Intervention (EMI) to encourage youth in learning and practicing CBT skills in their everyday lives (in vivo).

Methods: We developed an m-Health system called SmartCAT 2.0 (Smartphone-enhanced Child Anxiety Treatment) version 2.0, consisting of a smartphone application (app) for youth and an integrated clinician portal. The app contains: (1) a series of interactive games and activities to reinforce skill understanding, (2) an "in vivo" skills coach that cues the participant to use CBT skills during real-world emotional experiences, (3) a home challenge module to encourage home-based exposure, (4) a digital reward system that contains digital points and trophies, and (5) clinicianpatient messaging interface. Therapists use a secure Web-based portal connected to the app to setup required activities for each session, receive or send messages, manage child rewards and challenges, and view data and figures summarizing app's usage and skills coach entries. A usability study with five youth (aged 9 - 14) was conducted to measure app's effectiveness, efficiency, and satisfaction. Currently, a pilot study with ten (10) anxious youth (aged 9 - 14) receiving CBT treatment is still ongoing. The goal of the pilot clinical trial is to examine the platform's ability to effectively engage and motivate patient during treatment. Patient motivation will be evaluated based on engagement (time spent on app, the number of features initiated), retention (the number of app launch per day), the number of missed reminders, and proximity of the device from the youth.

Results: SmartCAT 2.0 was found to be capable of supporting CBT for child anxiety, and received positive feedback from both therapists and youth patients. Patients rated the app as highly usable (SUS Score $=78.5$ on $0-100$ range, Grade $=\mathrm{B}+$ ). Patient engagement measures will be reported when the pilot clinical trial is completed.

Conclusions: Patients found SmartCAT 2.0 both acceptable and easy to use. The integration of interactive games and gamification with CBT to support in vivo skills practice can increase patients' involvement in treatment.

\section{Objectives}

1. Understand challenges in CBT for anxious youth

2. Identify appropriate game elements/mechanics

3. Integrate game elements/mechanics to improve patient motivation in CBT treatment

\section{MONDAY, MAY 16, 2016}

1:15 PM-2:15 PM Monday, May 16, 2016

\section{Session 35 Session Title: ASYNCHRONOUS TELEPSYCHIATRY RESEARCH OUTCOMES}

MODERATOR: Tania Malik, JD, BA, Board Member

MindCare Solutions Group Inc, Nashville, TN

DESIGN AND ENGINEERING OF A LANGUAGE INTERPRETATION PLATFORM FOR ASYNCHRONOUS TELEPSYCHIATRIC AND LIVE IN-PERSON ENCOUNTERS

PRESENTER: Shang Wei, MS, Software Engineer / Data Manager, Steven R. Chan, MD MBA, Alberto Odor, MD MBA, Michelle Parish, MA, Peter Yellowlees, MD MBBS, Glen Xiong, MD, Andrés Sciolla, MD. University of California, Davis, Sacramento, CA

Introduction: Machine translation (MT) and automatic speech recognition (ASR) technologies can be used in both translation and interpretation: translation of static health education materials, and interpretation of face-toface and telemedical clinical encounters. MT technologies can be applied towards both synchronous and asynchronous telepsychiatry encounters, especially among those with different cultures. However, these technologies often exist as separate engines and components that are not directly usable for both asynchronous telemedicine and evaluating clinical effectiveness. Our project implements an efficient, provider compatible, administratively simple health IT solution: automated translation and transcription of Spanish spoken words recorded on video into English subheadings shown on the video recording of the original foreign language interview. This enables clinical assessment using asynchronous telepsychiatry consultations without the need for interpreters.

Methods: The platform interfaces with multiple MT engines - including Microsoft Translator, Google Translate, and IBM Watson Translation Service. To effectively use these translation services, we built a platform that can store usage data and abstract the programming interfaces of all MT engines. In addition, this platform accommodates various app clients and end-user devices, including iPhone and Android devices. The language interpretation platform was built on top of Node.js and MongoDB. Node.js is an event-driven web server based on Chrome's V8 JavaScript engine and MongoDB is a NoSQL, document-oriented database that supports JavaScript 


\section{ORAL PRESENTATIONS ABSTRACTS}

natively. The platform has a programmatic interface that any web applications or mobile apps can send the text to get translated. Key usage data like the original text, languages, translated text, and timestamps are stored in the database, so these data points can be used systematically for the study's accuracy analyses.

Conclusion: We discuss the creation of a language interpretation platform for use in asynchronous telemedicine and live in-person encounters. We will demonstrate the design, implementation, and use of an open-source clinical tool that will enable English-translated captions to appear in asynchronous telepsychiatry consultations. The voice recognition and language translation will occur automatically using software and can be checked for accuracy.

\section{REFERENCES}

1. Khanna RR, Karliner LS, Eck M, Vittinghoff E, Koenig CJ, Fang MC. Performance of an online translation tool when applied to patient educational material. J Hosp Med 2011; 6(9):519-25.

2. Balk EM, Chung M, Chen ML, Trikalinos TA, Kong Win Chang L. (2013) Assessing the Accuracy of Google Translate to Allow Data Extraction From Trials Published in Non-English Languages. Rockville (MD): Agency for Healthcare Research and Quality (US). Available from: http://www.ncbi .nlm.nih.gov/books/NBK95238

3. Sharif I, Tse J. Accuracy of computer-generated, Spanish-language medicine labels. Pediatrics 2010; 125(5):960-65.

\section{Objectives}

1. To explain the need for a language interpretation platform

2. To describe the current design and implementation of a language interpretation platform

3. To demonstrate various platform functionality via a web app

\section{ASYNCHRONOUS TELEPSYCHIATRY: A COLLABORATIVE CARE MODEL} AND A NEW PHYSICIAN SUPPORT ROLE

PRESENTER: Michelle B. Parish, MA, Research Manager; PhD Student, Steven Chan, MD, Lorin Scher, MD, Robert McCarron, MD,

Peter Yellowlees, MD

University of California, Davis, Sacramento, CA

Introduction: Collaborative care models in primary care improve care and lower costs, particularly for patients with chronic diseases and mental illness [1]. Within this model, coordinating patient care between specialty physicians, nurses, mental health professionals and primary care physicians is essential to improve patient care within the framework of the patient centered medical home [2,3]. Despite the promise of these models, collaborative models of care in mental health have not grown rapidly in the U.S. and most patient care continues to center around specialist models of care with limited collaboration between PCP and specialists and limited roles for physician support providers. The time burden PCPs are facing to manage growing panels of patients and their limited availability is now a legitimate concern as the rate of medical graduates specializing in primary care has dropped dramatically and will continue to decline over the next decade [1]. In this setting health information technologies such as telemedicine have been identified as important tools in advancing the PCMH by improving and streamlining communication between PCPs and specialists, in addition to increasing access to specialists [4]. In the specialty of psychiatry, the use of asynchronous models of telepsychiatry have been proposed to close the gap between PCP and specialist by increasing communication and by creating physician support roles to help PCPs manage patients with comorbid physical conditions and mental illness. Asynchronous telepsychiatry (ATP) is patient centered care that aims to increase access to care. The ATP consultations are recorded in the patient's primary care clinic by physician extenders which may save costs and increase the availability of appointments $[5,6]$.

Methods: We developed asynchronous telepsychiatry consultation to primary care which expands roles in healthcare for social workers and mental health therapists. The asynchronous telepsychiatry (ATP) clinical workflow enables primary care providers to receive psychiatric consultations for patients who they are managing mental health issues for in primary care. ATP consultations utilize a physician extender who is trained in psychiatric interviewing to conduct detailed mental health interviews with patients which are sent instantly via video to a consulting psychiatrist. The psychiatrist then reviews the data and sends a detailed consultation note back to the primary care provider. The interviewer uses in expensive and portable equipment to capture the video and securely send it to the psychiatrist for review. The ATP interview is a semi-structured interview based upon medical and clinical mental health interviewing [7] and guides we developed in our pilot research. We have standardized our ATP interview and with input from ATP clinicians and psychiatrists as part of a larger study. We will demonstrate the procedure of ATP interviewing and compare the inter-rater reliability of a subsample ATP interview notes across ATP clinician raters.

Results: We will present cohort data from the first 50 patients assessed by these interviewers. Each patient will have five interviews over two years during the course of the project and we will present data from the initial cohorts who have had up to three interviews each. We will present primary reliability data of ATP clinician raters.

Conclusions: We will present primary reliability data of ATP clinician raters. We will demonstrate the procedure of ATP interviewing and compare the inter-rater reliability of a subsample ATP interview notes across ATP clinician raters.

\section{REFERENCES}

1. Bodenheimer T, Chen E, Bennett HD. Confronting the growing burden of chronic disease: can the U.S. healthcare workforce do the job. Health Aff (Millwood) 2009; 28(1):64-74

2. Brett A, Blumberg L. Video-linked court liaison services: forging new frontiers in psychiatry in Western Australia. Australas Psychiatry 2006; 14(1):53-6.

3. Van Citters AD, Bartels SJ. A systematic review of the effectiveness of community-based mental health outreach services for older adults. Psychiatr Serv 2004; 55(11):1237-49.

4. Croghan T, Brown J. Integrating Mental Health Treatment Into the Patient Centered Medical Home. 2010, Agency for Healthcare Research and Quality (Prepared by Mathematica Policy Research under Contract No. HHSA290200900019IT02): Rockville, MD.

5. Yellowlees PM, et al. Transcultural psychiatry made simple asynchronous telepsychiatry as an approach to providing culturally relevant care. Telemed J E Health 2013; 19(4):259-64.

6. Butler TN, Yellowlees PM. Cost analysis of store-and-forward telepsychiatry as a consultation model for primary care. Telemed J E Health In Press.

7. Scher LM. Psychiatric Interview. Medscape, 2014.

\section{Objectives}

1. Describe standardized semi-structured asynchronous interviewing for psychiatric consultation to primary care

2. Present cohort data from the first 50 patients assessed by these interviewers

3. Present preliminary reliability data for asynchronous clinical interviewing 
Roundtable 1

Room 211 AB

MONDAY, MAY 16, 2016

1:15 PM-2:15 PM

Monday, May 16, 2016

\section{Session 84}

Session Title: ROUNDTABLE SESSION

\section{IMPLEMENTING A CONVERGED INPATIENT AND OUTPATIENT}

TELEMENTAL HEALTH PROGRAM

PRESENTER: Mark Berger, MBA, BA, Program Coordinator

CHI Franciscan, Tacoma, WA

Introduction: The goal of this presentation is to provide participants with the foundational information required to address the General, Clinical, Technical and Administrative / Financial requirements associated with implementing a TeleMental Health program. Additionally, the unique requirements and opportunities associated with a "Converged Inpatient \& Outpatient" program will be covered.

Background Narrative: CHI Franciscan faced numerous challenges and opportunities associated with cost effectively providing Mental Healthcare throughout the Puget Sound region. Limited access to Psychiatric Providers who are often centrally located at inpatient hospital units was a significant consideration. In 2013 the decision was made to develop and launch a Tele-Mental Health program. The program began with video "Cart" connectivity to one hospital for a single Psychiatrist conducting inpatient psychiatric consults. The program was deemed to be of value in meeting patient needs and was subsequently expanded to 3 additional hospitals. Approximately 30 Licensed Social Workers and 2 additional Psychiatrists have been added to the system. In January of 2015 the program was expanded to include Reimbursed Outpatient consults and is now serving 2 outpatient clinics. The Washington Parity Law comes into effect in 2017. We are in the process of developing a program expansion plan to fully leverage this exciting opportunity to meet our patient's mental healthcare needs.

Presentation Topics:

General Program Considerations

- Legislative Requirements (WA and in your state)

- Assess Organization Needs

- Champions \& Stakeholders

- "Process Mapping" ("swim lane" example to include ALL stakeholders)

- Deployment Plan

Clinical

- Mental Healthcare appointments conducive to Telemedicine

- Provider availability

- Types of available Providers who can provide care (i.e. Psychiatrists, Nurse Practitioners, LSW, Behavioral Analyst)

- "Tele-presentation" Requirements and Training

- Prescription processes

- Viable originating site options

- Charting

Technical

- Organization wide access to video conferencing

- Central Video Conference Platform

- Network Requirements

- Wide Area Network

$\circ$ Local Area Network / WIFI $\circ$ Internet (secure to homes)

- Video Endpoints (Carts, Fixed, Provider specific options)

- Workstation impacts

- Call Center Technology

- HIPAA Compliance

- Systems utilization reporting (call detail reports)

- EMR Development consideration (if part of IT)

Administrative / Financial

- Call Center Staff (receive / route calls, Schedule Pt. appointments)

- Patient Scheduling Management / Follow Ups

- "Reception" and Patient Registration

- Patient / Provider / Clinic Communications

- Payer Management

- Resource availability (Multi location: Provider, Site, Technology)

- EMR, Scheduling, Billing systems development

- Coding / Billing

- Reimbursement

Conclusion: Provide summary of lessons learned at CHI Franciscan to date and an update on emerging strategy.

\section{REFERENCE}

1. ATA, State Telemedicine Gaps Analysis, Coverage and Reimbursement; Latoya Thomas, Gary Capistrant; May 2015, pg 77.

Objectives

1. Identify key elements required for a Telemental Health program

2. Identify key Technical challenges and potential solutions for a successful program

3. Demonstrate awareness of legislative and organizational issues associated with successful program implementation

\section{Case Study Panel}

\section{MONDAY, MAY 16, 2016}

4:15 PM-5:15 PM

Monday, May 16, 2016

\section{Session 42 \\ Session Title: PRESCRIBING DURING TELEPSYCHIATRY PRACTICE: MODELS OF CARE FOR CHALLENGES ACROSS THE LIFESPAN}

MODERATOR: Kathleen M. Myers, MD, MPH, MS, Professor, Director Telemental Health

Seattle Children's Hospital, University of Washington, Seattle, WA

PRESENTER: Donald M. Hilty, MD, Professor, Vice Chair

Keck School of Medicine at USC, Los Angeles, CA

PRESENTER: Terry Rabinowitz, MD, DDS, Professor of Psychiatry and Family Medicine

University of Vermont College of Medicine, Burlington, VT

Few reports have addressed the challenges of providing pharmacotherapy for patients during telepsychiatry practice. No reports have addressed telepsychiatrists' fidelity to guideline-based care in prescribing. Different models of care are used depending on patient characteristics, resources at the patient site, medical needs, monitoring, and legal/regulatory guidelines. This panel will present prominent telepsychiatrists' experience in providing pharmacotherapy and research examining the delivery of pharmacotherapy. 


\section{ORAL PRESENTATIONS ABSTRACTS}

The presenters present their models of care in providing pharmacotherapy. They address three age groups (elders, adults, children) across three setting (nursing homes, primary care, and mental health/direct service settings). These nationally prominent telepsychiatrists describe their work in improving PCPs' ability to provide evidence-based care as a result of telepsychiatric consultation; telepsychiatrists' adherence to guideline-based care in providing direct service; challenges in working with patients and staff at nursing homes; legal and regulatory issues in prescribing across sites; interstate challenges, electronic prescriptions for amphetamines, and the Ryan Haight Act regulating the use of Schedule II medications. The panel addresses challenges and solutions, as well as provide an overview of issues in prescribing during telepsychiatry practice.

\section{REFERENCES}

1. ATA Practice Guidelines for Videoconferencing-based Telemental Health, 2009 (See also Yellowlees PM, Shore J, Roberts L. ATA Practice Guidelines for Videoconferencing-based Telemental Health. Telemed J E Health 16(10):1074-89.)

2. ATA Practice Guidelines for Video-based Online Mental Health Services. Tel eHealth September 722-730, 2013 (See also Turvey C, Coleman M, Dennison 0. ATA Practice Guidelines for Video-based Online Mental Health Services. Telemed J E Health 2013; 19(9):722-30.)

3. Fortney JC, Pyne JM, Mouden SB, Mittal D, Hudson TJ, Schroeder GW, et al. Practice-based versus telemedicine-based collaborative care for depression in rural federally qualified health centers: A pragmatic randomized comparative effectiveness trial. Am J Psychiatry 2013; 170(4):414-25.

4. Hilty DM, Ferrer DC, Parish MB, et al: The effectiveness of telemental health: A 2013 review. Telemed J E Health 2013; 19(6):444-54

5. Myers KM, Vander Stoep A, Zhou C, McCarty CA, Katon W. Effectiveness of a telehealth service delivery model for treating attention-deficit hyperactivity disorder: results of a community-based randomized controlled trial. J Am Acad Child Adolesc Psychiatry 2015; 54(4):263-74

6. Rockhill C, Tse JT, Fesenmeyer M, Gardia J, Myers KM. Telepsychiatrists' medication treatment strategies in the Children's ADHD Telemental Health Treatment Study(CATTS). J Child Adolesc Psychopharmacol ePub - Aug 10, 2015.

Objectives

1. To learn of the challenges and solutions to providing pharmacotherapy during telepsychiatry practice

2. To learn of three models of care for providing pharmacotherapy during telepsychiatry practice, across sites and patient characteristics

3. To learn of telepsychiatrists' adherence to guidelines of care in providing pharmacotherapy

\section{TUESDAY, MAY 17, 2016}

11:00 AM-12:00 PM Tuesday, May 17, 2016

\section{Session 50 Session Title: EFFECTIVENESS OF AUTOMATED SPEECH RECOGNITION APPS}

MODERATOR: Scott Baker, Business Innovations Manager InSight Telepsychiatry, Marlton, NJ

NATURAL LANGUAGE INPUTS FOR MEDICATION RECONCILIATION: A FEASIBILITY STUDY

PRESENTER: Marquise Hopson, BS ${ }^{1}$, Neal Sikka, $\mathrm{MD}^{2}$, Charles Jankowski, $\mathrm{PhD}^{3}$, Ann Thyme-Gobbel, $\mathrm{PhD}^{3}$
${ }^{1}$ The George Washington University, Washington, DC, ${ }^{2}$ The George Washington University - Medical Faculty Associates, Washington, DC, ${ }^{3}$ Gamgee, Inc, San Mateo, CA

Introduction: Medication errors and adverse events are common throughout the healthcare continuum, often at transitions of care. The clinical and financial impact of these events is significant, both to the patient and the healthcare system. To decrease medication errors and adverse drug events. In 2005, The Joint Commission identified medication reconciliation as National Patient Safety Goal (NPSG) No. 8 in an effort to reduce errors and subsequently improve patient outcomes. Our study aims to address medication reconciliation using an innovative approach that leverages a mobile natural language processing (NLP) software application for vocal medication intake. A physician focused vocal medical dictionary was tested with laypersons for feasibility. We captured recordings of medications spoken in a variety of dialects and accents from laypersons in order to determine the accuracy and sensitivity of voice recognition with natural language processing in various environmental settings. The future goal is to develop an application that allows medications spoken from patients directly into the EHR or PHR.

Methods: The study protocol was approved by the IRB. We recruited and interviewed a convenience sample of adult volunteers (18-99 years old) on the National Mall in Washington, DC, who spoke English. Once eligibility was verified, participants completed a demographics survey that also include questions related to smartphone use and healthcare utilization. Participants were then asked to recall up to five medications, supplements, or vitamins of their choosing to speak into the IOS mobile application run on an iPhone. Participants then spoke out loud the medications into the device microphone. The application processed the input first by recognizing the speech from the voice recognition engine and then matching recognized medications with a grammar derived from a limited National Drug Code (NDC) directory of approximately 75,000 medications. If identifiable, the screen displayed a text listing the medication names spoken. All captured voice utterances were transcribed to allow detailed and accurate data analysis.

Results: Forty-seven participants were recruited and forty-six inputs were suitable for analysis. Participants represented seventeen US states, the District of Columbia, and three countries outside of the US. Their median age at 47 51\% were male, $87 \%$ were native English speakers, 32\% were black, $62 \%$ white, and 4\% Asian. 40\% of the participants had completed college and 96\% have access to a smartphone in their household. In the last 12 months 15\% reported at least one hospitalization, 17\% at least one ED visit, and 67\% at least one outpatient visit. A significant number of participant records contained environmental noise and/or side speech, making the task challenging for the software. We used industry standard measures for speech recognition Word Error Rate (WER) and Information Retrieval measure of Precision and Recall, in order to gauge the accuracy of the application at recognition medications. Without accounting for medications spoken that were not listed in the NDC directory, the application had a Recall rate of 97\% with a Precision of $77 \%$ and a WER of $29 \%$. Including only identifiable medications, recall remained $97 \%$ and Precision increased to $82 \%$.

Conclusions: The results of this feasibility study suggest that the use of voice recognition and NLP for medication intake can be effective. We found that even with a variety of layperson inputs recorded against a wide range of background noise levels, the NLP software was able to accurately detect medication inputs from laypersons with high precision and recall rates. Some drug names spoken by participants were listed differently in the NDC directory and therefore not identified. Often laypersons call medications by a varied or partial terms that do not match the NDC list exactly, limiting recall. In particular, future methods for generating a grammar from the NDC directory will add terms for generic medications excluding final chemical compounds, as this is a frequent mode of speaking medications. In future testing of the software, we plan to include more common names and variations of medi- 
cations to improve recognition. We also want to control ambient noise and side speech by testing with real patients in task-appropriate environments to include the home, clinic or hospital. Finally, we want to further understand patient satisfaction, usability, and clinical efficacy in capturing and reconciling medication lists using vocal intake applications.

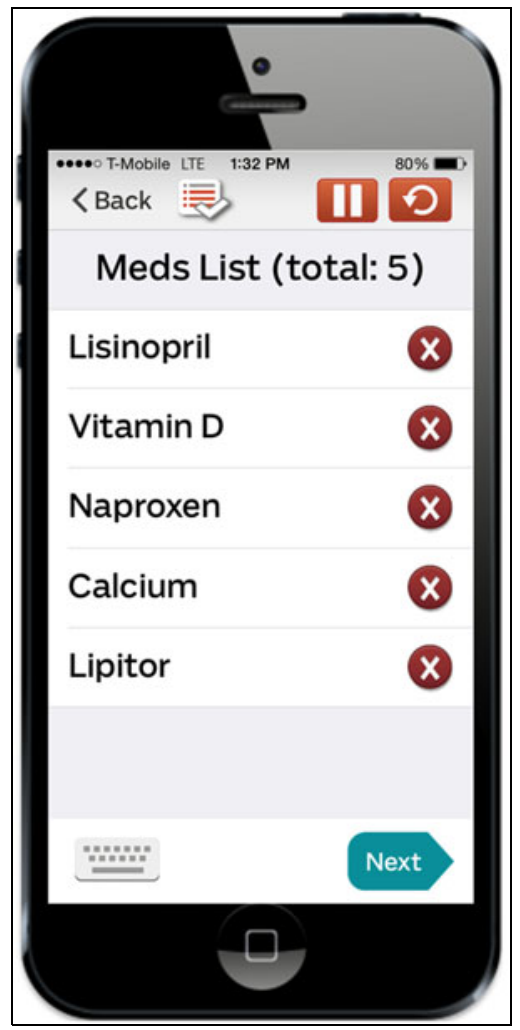

\section{REFERENCES}

1. Gleason KM, McDaniel MR, Feinglass J, et al. Results of the medications at transitions and clinical handoffs (match) study: An analysis of medication reconciliation errors and risk factors at hospital admission. J Gen Intern Med. 2010; 25(5):441-47.

2. Greenwald JL, Halasyamani L, Greene J, et al. Making inpatient medication reconciliation patient centered, clinically relevant and implementable: A consensus statement on key principles and necessary first steps. J Hosp Med. 2010; 5(8):477-45.

3. Centers for Medicare \& Medicaid Services. Stage 2 Eligible hospital and critical access hospital meaningful use core measures: measure 11 of 16. 2012:1-2. http:// www.cms.gov/Regulations-and-Guidance/Legislation/EHRIncentivePrograms/ downloads/Stage2_HospitalCore_11_MedicationReconciliation.pdf. Accessed May 23, 2015.

4. Nuance Communications I. Physician Speech Recognition Solutions. http:// www.nuance.com/products/physician-speech-recognition-solutions/ index.htm?ref=footer. Accessed May 23, 2015.

\section{Objectives}

1. Discuss challenges in medication reconciliation

2. Describe a mobile health application of natural language processing for medication intake
3. Analyze data related to effectiveness of vocal intakes for medication capture

ERROR RATES IN AUTOMATED MACHINE TRANSLATION AND SPEECH RECOGNITION IN TELEPSYCHIATRY PATIENT-CLINICIAN ENCOUNTERS

PRESENTER: Steven R. Chan, MD, MBA, Senior Resident Physician, Shang H. Wei, MS, Michelle Parish, MA, Glen Xiong, MD, Andrés Sciolla, MD, Peter Yellowlees, MD MBBS

University of California, Davis, Sacramento, CA

Introduction: Although machine translation (MT) and automatic speech recognition (ASR) technologies have been used for the translation of static health education materials, videoconferencing, and translation of face-toface clinical encounters, they have not previously been clinically validated in the known research literature. The use of such technologies could potentially boost access for limited English proficiency (LEP) patients who are more proficient at speaking a foreign language, thus decreasing language disparities.

Methods: Our current research in automated speech recognition and machine translation software tests whether such technologies are usable by healthcare providers. Our platform uses multiple commercially-available translation engines, designed to be portable and run on a wide array of hardware. We have created translations of 1) public health materials and patient education literature, 2) publicly-available patient interview videos, and 3) English and Spanish clinician-patient interview videos from our team's asynchronous telepsychiatry study, and compared them with translations generated by human interpreters. Error rates of MT and ASR will be compared with transcripts generated by human medical interpreting services. The accuracy will measure particular translation errors following the research of Glenn Flores et al in medical interpretation: omission, in which the interpreter (human or machine) did not interpret a word/phrase uttered; addition, in which the interpreter added a word/phrase not uttered by the patient; substitution, in which the interpreter substituted a word/phrase for a different word/phrase; and false fluency, in which the interpreter used an incorrect word/phrase that does not exist in that particular language. Back-translation (also known as round-trip translation, or RTT) will be used for Englishspeaking patients by translating to Spanish, then back to English, and will then be measured for the aforementioned error rates. Error rates are also computed using automated machine translation evaluation algorithms, including word error rate.

Results: Pending.

Conclusions: Initial results of our accuracy analyses of speech recognition and translation engines, based on computed error rates and semantic differences, will be discussed. The use of a translation platform as used in a live patient encounter and a recorded asynchronous telepsychiatry encounter will also be demonstrated.

\section{REFERENCES}

1. Flores $G$, Abreu M, Barone $C P$, Bachur $R$, Lin H. Errors of medical interpretation and their potential clinical consequences: a comparison of professional versus ad hoc versus no interpreters. Ann Emerg Med 2012 60(5):545-53.

2. O'Neill DD, Anthony S, Laws M. (2013). Every Language Now. In L. Berkowitz \& C. McCarthy (Eds.), Innovation with Information Technologies in Healthcare (pp. 167-177). London: Springer London.

3. Ohtani A, Suzuki T, Takeuchi H, Uchida, H. (2015). Language Barriers and Access to Psychiatric Care: A Systematic Review. Psychiatr Serv 2015; 66(8): 798-805. 
4. Wilson CC. Patient safety and healthcare quality: the case for language access. Int J Health Policy Manag 2013; 1(4): 251-53.

\section{Objectives}

1. To describe the advantages and disadvantages of automated speech recognition and machine translation software, and contrast this with other telemedicine interpretation technologies and in-person human interpreters

2. To apply speech and translation technologies to telepsychiatry, patientdoctor encounters, and patient education materials

3. To analyze error rates and describe issues stemming from use of speech and translation technologies

\section{TUESDAY, MAY 17, 2016}

\section{1:15 PM-2:15 PM Tuesday, May 17, 2016}

\section{Session 58 Session Title: CASE STUDIES IN TELEMENTAL HEALTH}

MODERATOR: Kimberly Sloan, PhD, Clinical Psychologist

St. Charles West Psychological, Mt Pleasant, SC

\section{TELEMEDICINE FOR PRISONS: HOW CALIFORNIA BUILT A THRIVING} PROGRAM

PRESENTER: Edward Kaftarian, MD, Chief of Telepsychiatry California Department of Corrections, Pasadena, CA

Prepare to embark on an eye-opening tour through the California prison telepsychiatry system. See how telepsychiatry helps buoy the one of the largest prison mental health systems in the country.

We will begin with a brief overview of the California prison system healthcare program. We will also discuss the national incarceration trends and explore how this affects the future of healthcare.

We will then explore the colorful history of how telepsychiatry was established within a large government agency. We will reveal the challenges and opportunities in correctional telehealth. This brief but entertaining journey

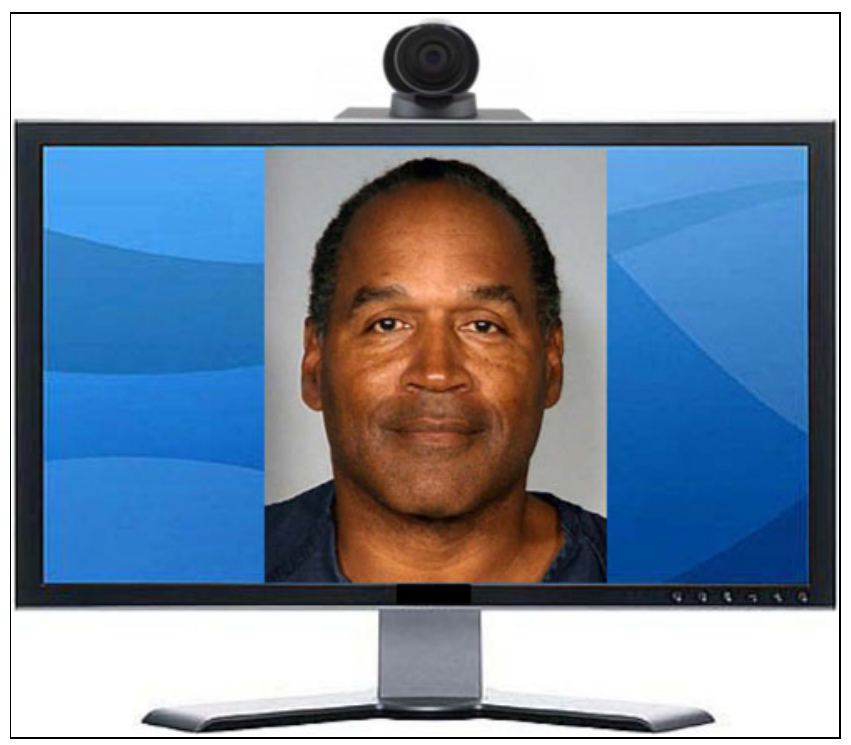

will be exciting and inspiring for anyone interested in developing a correctional telehealth program and delivering hope to the castaways of society.

Objectives

1. Examine and describe the steps needed to create and develop a telehealth program for the correctional population

2. Describe the population trends in the incarcerated population and discuss how this affects the need for telemedicine

3. Formulate strategies for overcome unique obstacles that correctional environments pose to telehealth

\section{UNINTENDED CONSEQUENCES: TELE-PSYCHIATRY \\ AS AN AGENT OF CHANGE}

PRESENTER: Deborah Wedick-Cooper, BS, RN, CNRN, Dignity Health ${ }^{1,2}$ ${ }^{1}$ Mercy Medical Center Redding, Redding, CA, ${ }^{2}$ Dignity Health, Redding, CA

Mercy Medical Center Redding (MMCR), implemented an ED psychiatric crisis telehealth service July 1, 2014. The goal for this service line was a reduced LOS for mental health patients and an overall improvement in ED total throughput times. It was anticipated that the services would see approximately 25 patients a month initially with a sustained goal of 35-50 consults monthly. The return on investment was justified by a reduction in opportunity costs for use of beds for non-billable services.

Early data showed a modest trend line towards LOS reduction and a increasing demand for behavioral health services. By the middle of the fiscal year, MMCR was the largest mental health service line for a non-designated facility in the Dignity Health Telemedicine Network, extremely popular with law enforcement as a drop-off and LOS was trending upward.

Further impact on the project was the delay in county services and the refusal of the county to grant LPS 5150 rights to the ED or Telehealth psychiatrists who were licensed and board certified in their specialty. Patients would be rapidly evaluated, have medications started, but could not be discharge without the approval of a county worker. Those requiring an inpatient stay had to be placed by the county. California in-patient psychiatric beds are at a premium (reference) beds are at a premium. When no beds were available, patients were boarded in the ED for days with no mechanism for treatment or recouping the cost of boarding. It was time to regroup and check our original assumptions and data sources.

Our initial data and tracking mechanism was based up a compiled synopsis for the daily ED activity log. When compared to an analysis of the log itself, there were very different numbers which gave an entirely different picture of ED activity. Long term psychiatric boarders were washed out of the LOS averages as outliers. Many psychiatric patients were missing from the totals as they were entered without identifiers that would include them in the appropriate groups. No record of county response times was tracked and their stated four to six hour response time was determined to be aspirational at best.

We validated our findings with the other hospital in Redding also operating a brand new tele mental health service who confirmed our hypothesis that the problem of crisis mental health service delivery in our community was far more extensive than the original data indicated. We shared our data and found nearly identical outcomes. We identified the key barrier to success for our original goal of reducing mental health LOS: Shasta County control of LPS 5150 status.

Over the next six months we met with the county to escalate community and hospital pressure to allow tele-psychiatrists parity with the county employees. According to our current statute, a Mental Health Technician can override a psychiatrist who is not designated by Shasta County when it comes to the disposition of a patient. We have had a number of instances when the tele-psychiatrist has suggested that the patient be referred for inpatient treatment and the patient has been discharged by the county worker. Two cases resulted in suicide.

Our first year of active delivery of mental health services via telemedicine has been challenging and educational. We have come face to face with the dys- 
functional system of behavioral health services in California which is beyond the remedy of any quick fix, flip a switch telehealth program. We have identified the staggering real opportunity costs incurred by our institution and the disproportional use of resources needed to handle mental health patients in the ED. We have also established best practices, a care timeline, a medication formulary for telehealth patients and discharge standards consistent for the entire community. We are now pending an agreement with the county for parity for our tele-psychiatrists for LPS designation, access to the Anazasi data base for treatment history and a joint data site for review of case outcomes. We have leveraged our telehealth service from a simple gap analysis filler to a dynamic change agent.

Be careful what you wish for.

\section{REFERENCES}

1. CA Overview (by County) of Behavioral ED Growth (2006-2011) - likely \% will be higher if looking at more recent years https://medamerica.box.com/ CABehavioralHealthEDGrowth

2. Psychiatric Boarding in U.S. EDs: A Multifactorial Problem that Requires Multidisciplinary Solutions by Urgent Matters, 2014 - provides a good overview of the psych ED boarding issues and potential solution, such as a PES: https:// medamerica.box.com/PsychEDBoardingMultiSolutions

3. The Banner Psychiatric Center: A Model for Providing Psychiatric Crisis Care to the Community while Easing Behavioral Health Holds in Emergency.

4. Little-Upah P, Carson C, Williamson R, et al. The Banner Psychiatric Center: A Model for Providing Psychiatric Crisis Care to the Community while Easing Behavioral Health Holds in Emergency Departments. The Permanente Journal 2013; 17(1):45-49.

\section{Objectives}

1. Identify and prioritize key decision points for process modification

2. Aline and maintain budgetary and regulatory compliance components of the service line

3. Predict potential consequences of scale

\section{TUESDAY, MAY 17, 2016}

\section{3:00 PM-4:00 PM Tuesday, May 17, 2016}

\section{Session 66}

\section{Session Title: A CHILD AND ADOLESCENT TELEMENTAL CLINICAL GUIDELINE: NEED FOR, OVERVIEW OF, AND COMPONENTS OF THE GUIDELINE}

MODERATOR: Kathleen Mary Myers, MD, MPH, MS, Professor, Director Telemental Health

Seattle Children's Hospital and University of Washington, Seattle, WA

PRESENTER: Donald M. Hilty, MD, Professor and Vice-Chair Keck School of Medicine at USC, Los Angeles, CA

PRESENTER: Eve-Lynn Nelson, PhD, Professor and Director University of Kansas Medical Center, Fairway, KS

PRESENTER: Terry Rabinowitz, MD, DDS, Professor of Psychiatry and Family Medicine

University of Vermont College of Medicine, Burlington, VT

The American Telemedicine Association (ATA) is the principal organization bringing together telemedicine providers, healthcare institutions, vendors and others involved in providing remote healthcare using telecommunications. Diverse groups from traditional medicine, academia, technology and telecommunications companies, e-health, allied professional and nursing associations, medical societies, government and others collaborate to overcome barriers to the advancement of telemedicine, including an effort to establish practice guidelines and technical standards, and to assure the uniform quality of service to patients. Work groups that include experts from the field and other strategic stakeholders put forward the preliminary guidelines work, which undergoes a thorough consensus and rigorous review, with final approval by the ATA Board of Directors. Adult telemental health (TMH) guidelines help practitioners make clinical, technological and administrative adjustments, but a child and adolescent TMH guideline is needed due to differences between child/adolescent and adult populations. Now that approximately 50 studies have generated significant evidence, we can build on adult guidelines and pre-existing practice parameters and key considerations for children and adolescents. Modifications of existing general guidelines appear necessary, e.g., based on developmental status, family involvement, and patient-site modifications for space and sound. Child telemental health often involves collaborating across systems of care (e.g., schools, primary care practices, foster care, juvenile corrections, etc.) which require consideration. Additional clinical issues include who, exactly, is the "patient" (i.e., the patient, family and /or other stakeholders), modalities of care (i.e., age-related psychotherapies such as play therapy or behavior management), and psychopharmacology. The guidelines encompass clinical practice across settings, the infrastructure and staffing, child-specific ethical-legal considerations, and the management of mental health emergencies. This new clinical guideline for TMH care of these young patients will help clinicians learn the evidence base supporting TMH practice with youth and to adapt his or her practice accordingly.

\section{REFERENCES}

1. ATA Practice Guidelines for Videoconferencing-based Telemental Health, 2009 (See also Yellowlees PM, Shore J, Roberts L. ATA Practice Guidelines for Videoconferencing-based Telemental Health. Telemed J E Health 16(10):1074-89).

2. ATA Practice Guidelines for Video-based Online Mental Health Services. TeleHealth September 722-730, 2013 (See also Turvey C, Coleman M, Dennison 0. ATA Practice Guidelines for Video-based Online Mental Health Services. Telemed J E Health 2013; 19(9):722-30).

3. Myers K, Cain S. Practice Parameter for Telepsychiatry with Children and Adolescents. J Am Acad Child Adolesc Psychiatry 2008; 247(12):1468483.

4. Hilty DM, Shoemaker EZ, Myers KM, et al. Need for and steps toward a clinical guideline for telemental healthcare of children and adolescents. J Child Adol Psychopharm, ePub - Feb 12, 2016.

5. Myers K, Turvey C: Telemental Health: Clinical, Technical, and Administrative Foundations for Evidence-Based Practice, Elsevier Press, 2013.

\section{Objectives}

1. Summarize the need for and steps toward formal child and adolescent TMH guidelines and a synopsis of how guidelines are developed

2. Provide both an outline and rationale of key components of child and adolescent TMH guidelines

3. Discuss "real-world" clinical, administrative, and technical issues related to TMH for clinicians

\section{TUESDAY, MAY 17, 2016}

4:15 PM-5:15 PM Tuesday, May 17, 2016

\section{Session 74}

\section{Session Title: HOW-TO TELEMENTAL HEALTH IN NON-INSTITUTIONAL SETTINGS}

MODERATOR: Donna Dittman Hale, MHA, Executive Director Bay Rivers Telehealth Alliance, Tappahannock, VA 


\section{ORAL PRESENTATIONS ABSTRACTS}

\section{ACT NOW FOR INNOVATION: DEVELOP A TELEMENTAL HEALTH PROGRAM FOR ACT TEAMS}

PRESENTER: Shelley Sellinger, MD, Scheduled Telepsychiatrist InSight Telepsychiatry, Marlton, $N J$

PRESENTER: Laura Marvel, Unit Director, Delaware ACT - ICM Resources for Human Development, Dover, DE

The shortage and unequal distribution of behavioral health providers especially prescribers - combined with the growth of the telemedicine industry, has led to the emersion of innovative telemental programs. One such program has developed in Delaware, where ACT teams are now using 4Genabled tablets to connect to remote psychiatrists while other providers are onsite in individuals' homes.

This case study presentation details perhaps the nation's first ACT program to utilize telepsychiatry and addresses the operational, community and regulatory buy-in necessary for a program to succeed.

Assertive Community Treatment (ACT) has been successful in treating individuals who have recently transitioned from inpatient care by facilitating collaboration between all of a consumer's mental health providers. Studies show that it is extremely effective and can reduce hospital days by up to $78 \%$ for outpatient clinical care. However, with a diverse and rare team, it becomes difficult and costly to bring everyone together frequently. To alleviate this challenge and connect remote ACT providers, Resources for Human Development (RHD) and InSight Telepsychiatry partnered to apply telemental health to an existing ACT program in Delaware.

First, the team established an unshakable proof of concept of telepsychiatry's efficacy for scheduled telepsychiatry services like those commonly offered within general community mental health outpatient clinics. Then RHD and InSight came together to find where more telemedicine innovation could fit into their programming and expand their psychiatric reach. RHD's ongoing ACT programs were the answer. Following a similar proof of concept approach, the partners first brought telepsychiatry to ACT consumers within RHD's established clinic sites under a model where ACT consumers were transported to RHD's sites to meet with their psychiatrist virtually via technology at the clinic. After having established the efficacy and acceptability of telepsychiatry with this team approach on behalf of ACT consumers, RHD and InSight sought to really maximize the power of the technology to bring psychiatric services directly to ACT consumers in their homes.

In order for this program to come to fruition, leaders at RHD and InSight had to cultivate buy-in from team members, consumers, providers, regulators, and state leaders at many levels. This case study highlights the behind the scenes work required to build and launch such an innovative program.

Key stakeholders involved in making this particular program happen included the Delaware Department of Health and Social Services, the Division of Substance Abuse and Mental Health, the Delaware Medical Society, the Delaware Board of Medical Licensure and Discipline, the Delaware Telemedicine Coalition, the Division of Medicaid and Medical Assistance, The Mid-Atlantic Telemedicine Resource Center and leadership from the American Telemedicine Association, all of whom worked together to lay a framework for the program. This collaboration resulted in regulatory updates and approvals, the revision of the state's Medicaid waiver, and statewide reimbursement from Medicaid and private insurers for many telemedicine services. The result was a forward-thinking structure for future programs and a successful pilot of an innovative ACT program. Today, Delaware has become a stand out example of a state acting to fully embrace telemedicine in order to improve its services and bring care to its citizens.

This case study will discuss the collaboration between these stakeholders to foster an environment for telemedicine innovation and will also cover some of the operational best practices for implementing telemental health into ACT programs. This will include tips for the technical setup and training needed for remote and onsite providers to adapt their clinical approach. We will also highlight new clinical indicators made available to the telemedicine team working within consumers' homes.

As our industry moves more and more toward direct-to-consumer models of telemedicine, the facilitated in-home services of ACT programs offer many valuable lessons on how we adapt our facility-based services to really leverage the power of telemedicine technology within home environments.

Led by one of the program designers and key influencers involved in the ACT telemental health program, this case study will give leaders tangible tools for developing similarly innovative models.

\section{REFERENCES}

1. Latimer, E. (2005). Economic considerations associated with assertive community treatment and supported employment for people with severe mental illness. Journal of Psychiatry and Neuroscience, 30(5), 355-359. Retrieved from http://www.ncbi.nlm.nih.gov/pmc/articles/PMC1197281

\section{Objectives}

1. Identify the buy-in needed to develop new telemedicine programs

2. Demonstrate the ways behavioral healthcare organizations can collaborate in order to develop innovative programs

3. Explain how multiple providers can collaborate in practicing via direct-to-consumer telebehavioral health

\section{FAST-PACED WORK FROM THE COMFORT OF HOME: CLINICAL CONSIDERATIONS FOR CRISIS TELEPSYCHIATRY}

\section{PRESENTER: Doug Ikelheimer, MD, On-Demand Telepsychiatrist InSight Telepsychiatry, Marlton, $\mathrm{NJ}$}

One model of telepsychiatry that is growing in popularity, but has received little attention to date, is crisis telepsychiatry. Crisis psychiatric providers who practice telepsychiatry immerse themselves in the buzz of a hospital emergency department, mobile health crisis units, ACT programs or other crisis settings from the comforts of their home office. By "beaming in," they offer much-needed, on-demand psychiatric expertise that can reduce the amount of time consumers in crisis wait for proper care.

For the organizations that crisis psychiatric providers serve via telepsychiatry, the benefit of timely access to psychiatric assessment and expertise is immeasurable. Utilization of crisis telepsychiatry results in consumers receiving the least restrictive and most appropriate level of care quickly, even on tough-to-staff but high-volume nights and weekends when the majority of psychiatric crisis occurs.

This presentation will review the training, setup and practical considerations needed to begin work as a remote crisis telepsychiatrist. Learning to adapt your clinical approach to translate well through technology requires communication adjustments, new environmental and cultural considerations and, most of all, practice. It is also vital to be thoroughly trained and comfortable with the video technology, remote connection tools and EMRs they will be using to serve consumers. This presentation will discuss tips for collaborating with onsite clinicians and navigating the murky waters of collecting collateral, ordering tests, making community referrals, writing prescriptions and staying up to date on cultural, community and organizational nuances all while practicing remotely.

For the health-administrators in the room, this presentation will give tips and best practices for developing and implementing a successful crisis telepsychiatry program through the lens of a crisis telepsychiatry provider who has gone through the hurdles and hoops of implementation, credentialing, training and care giving with over 10 crisis telepsychiatry programs.

Lastly, after establishing the foundation for appropriate, proper crisis telepsychiatry from an informed and engaged remote physician, this presentation will look at several crisis telepsychiatry case studies and review how a practicing telepsychiatrist dealt with each unique consumer encounter via 
televideo. This section will discuss practical lessons learned while weaving in clinical research studies surrounding the efficacy and best practices for telepsychiatry with several different consumer-populations.

This session will give crisis telepsychiatry hopefuls a comprehensive perspective on all of the considerations necessary to delve into this booming form of care.

\section{REFERENCE}

1. Williams, M., Pfeffer, M., Boyle, J. (2009). Telepsychiatry in the Emergency Department: Overview and Case Studies. California HealthCare Foundation. Retrieved from http://www.securetelehealth.com/images/downloads/edtelepsych.pdf

\section{Objectives}

1. Compare the benefits and hurdles of crisis telepsychiatry

2. Recall the training and resources needed to start practicing telepsychiatry

3. Apply telepsychiatry clinical best practices to their own remote consumer experiences

\section{Track: Pediatrics Room 208 AB}

\section{SUNDAY, MAY 15, 2016}

12:00 PM-1:00 PM Sunday, May 15, 2016

\section{Session 6 \\ Session Title: BEST PRACTICES IN PEDIATRIC TELEHEALTH}

MODERATOR: Kathleen Webster, MD, MBA, Director of Telemedicine Advocate Children's Hospital, Oak Lawn, IL

\section{TELEMEDICINE USE TO SUPPORT ORTHOPEDIC AND SLEEP MEDICINE PEDIATRIC SUBSPECIALTY CARE IN RURAL AREAS}

PRESENTER: John Garcia, MD, Pediatric Sleep Medicine,

Tom Novacheck, MD

Gillette Children's Specialty Healthcare, St. Paul, MN

Children with medical complexity (CMC) have multiple chronic conditions, require care from specialists and service providers to maintain optimal health, and often require life-sustaining technology. ${ }^{1} \mathrm{CMC}$ comprise less than $1 \%$ of U.S. and Canadian children, but account for disproportionately higher costs of healthcare. ${ }^{1}$ While technological advances have increased life expectancy and number of CMC, data from the American Association of Pediatrics indicates declining pediatric medical and surgical subspecialists, with most practicing in urban/academic settings. ${ }^{2}$ For CMC living in rural areas, accessing subspecialty care often requires extensive travel, family disruption and high out-of-pocket cost.

Gillette Children's Specialty Healthcare (GCSH), a sub-specialty system located in St. Paul, Minnesota, specializes in the care of complex child-onset conditions such as cerebral palsy, spina bifida, muscular dystrophies, epilepsy, and congenital syndromes. GCSH includes a 60-bed hospital and four outpatient clinics in the St. Paul metropolitan area. In addition, 14 outreach clinics located throughout Minnesota provide pediatric subspecialty care to rural children and are staffed by GCSH providers who travel from the St. Paul location.

This case study describes a telemedicine program between GCSH subspecialists and CMC living in rural Midwestern locations. Sleep medicine and orthopedics subspecialties were approached by rural non-GCSH physicians to provide additional outreach clinics that increase subspecialty care and decrease travel distance for rural families. With organizational support, telemedicine as an alternative to in-person outreach clinics was investigated, and robust clinic-to-clinic telemedicine consultation programs evolved. Both programs primarily serve children with cerebral palsy. Cerebral palsy carries an increased risk of sleep disorders in general and obstructive sleep apnea in particular, due to tone abnormalities that increase the risk of upper airway collapse. Cerebral palsy often results in spasticity and complex movement disorders requiring orthopedic surgical intervention.

Logistics for the programs vary. Both conduct monthly telemedicine clinics, utilize similar equipment (Polycom ${ }^{\mathrm{TM}}$ ), and the distant or hub site is the St. Paul GCSH outpatient clinic. What differs is duration of the program (years), originating site, telepresenter, assessment type, and number of in-person visits replaced by virtual visits. While both programs serve only children, neither originating site meets Medicare rural HPSA and MSA designations. However, families travelling to originating sites come from rural HPSA designations.

The pediatric sleep medicine program began in 2012 and serves 50-100 $\mathrm{CMC} /$ year. Typical consultation includes initial visit to assess all types of sleep disorders, and if obstructive sleep apnea is suspected an overnight polysomnogram at the St. Paul location is pursued. In this program, the initial visit is done via telemedicine. The originating site is a GCSH outreach clinic in northern Minnesota, 150 miles from St. Paul. The telepresenter is the GCSH outreach clinic nurse and except for turning on the telemedicine equipment, the visit protocol is identical to an in-person clinic visit.

The pediatric orthopedics program began in 2005 and serves approximately 50-60 CMC/year. Typical consultation includes initial visit to assess need for and type of orthopedic intervention, hospitalization at GCSH for surgical procedure, and post-operative follow-up at one, three and six months. Telemedicine replaces the initial assessment and two post-operative visits. The originating site is a non-GCSH physical medicine and rehabilitation (PM\&R) clinic in an adjacent state, 250 miles from St. Paul. The telepresenter is a local clinic physiatrist, responsible for overall management of the child's condition. Hands-on assessment is essential during orthopedic consultation. To support this component, the telepresenter visited the GCSH (distant site) provider to acquire unique physical assessment skills. The orthopedic specialist visited the originating site prior to program implementation and visit protocol guidelines were developed jointly.

Success is credited to organizational support that reduces barriers. ${ }^{3} \mathrm{GCSH}$ physicians conduct telemedicine visits in a St. Paul clinic room, with minimal workflow disruption. Trust between distant and originating sites is essential. The distant site must trust the accuracy of the originating sites physical assessment. The originating site must trust the ability of the distant site to provide optimal patient services with a focus on co-management rather than co-opting of patients. Face-to-face meetings prior to program initiation established this trust. Sustainability is ensured by billing all visits with telemedicine CPT modifier for optimal reimbursement.

Both programs reduce organizational and family costs while increasing access to subspecialty care. Substituting in-person with virtual clinics reduces organizational travel expenses by approximately $\$ 500 /$ clinic and lost billable time by approximately $\$ 3,000 /$ clinic. For families, out-of-pocket travel expenses are reduced by approximately $\$ 700 /$ visit. For both, the danger of travel is reduced. Children served by the programs often rely on equipment for mobility, communication, nutrition and breathing, and the potential for equipment failure during long periods of travel puts the child at risk. The telemedicine program is a 'win-win' situation for providers, CMC and their families. Similar to telehealth study in New Zealand, this program allows subspecialists to serve a wider geographic area while substantially reducing travel burden for providers, families and children. ${ }^{4}$

\section{REFERENCES}

1. Berry JG, Agarwal R, Cohen E, Kuo DZ. (2013). The landscape of medical care for children with medical complexity. Overland Park, KS: Children's Hospital 


\section{ORAL PRESENTATIONS ABSTRACTS}

Association. Retrieved from https://www.childrenshospitals.org/issues-andadvocacy/children-with-medical-complexity/issue-briefs-and-reports/thelandscape-of-medical-care-for-children-with-medical-complexity

2. Committee on Pediatric Workforce. Pediatrician workforce policy statement Pediatrics 2013; 132(2):390-97.

3. LeRouge C, Garfield MJ. Crossing the telemedicine chasm: Have the U.S. barriers to widespread adoption of telemedicine been significantly reduced? Int J Environ Res Pub Health, 2013; 10(12): 6472-484.

4. Rowell PD, Pincus $P$, White $M$, Smith AC. Telehealth in paediatric orthopaedic surgery in Queensland: A 10-year review. ANZ Journal of Surgery 2014; 84(12):955-59.

\section{Objectives}

1. Discuss characteristics of a successful urban-rural, clinic-to-clinic, pediatric subspecialty telemedicine program

2. Describe development of telepresenter skills for high-touch orthopedic examinations

3. Assess applicability of replacing in-person subspecialty clinic visits with virtual visits for rural populations

IMPLEMENTING VIRTUAL CARE FOR REMOTE SITE PEDIATRIC CRITICAL CARE EVENING ROUNDS

PRESENTER: Dwight Bailey, D0, Levine Children's Hospital Carolinas Healthcare System, Charlotte, NC

National and regional healthcare reorganization has led to the necessity of an increasingly efficient and effective means of patient care. Given the climate of today's healthcare system challenges, the pediatric critical care division of an academic, tertiary care children's hospital has expanded coverage to a regional Pediatric Intensive Care Unit (PICU) and driven the innovation to create a PICU telemedicine initiative. Equipment, cost, staff satisfaction and inter-hospital relationships were all considered for improvement and innovation in practice.

Our project mission was to explore the use of telemedicine technology between two regional PICUs to improve the patient rounding experience.

We aim to enhance team communication and plan of care via telemedicine that allows for visual-verbal contact between the two sites.

A team was developed to assess national guidelines and recommendations, review best practice, investigate equipment availability and assess this project against system goals for quality patient care. The multiphase project included implementation of a telemedicine cart within the regional PICU to allow high quality, 24/7 consultation, the creation of collaborative standardized telemedicine protocols for admissions, existing patient assessment and problem management, and nightly multidisciplinary rounding. These processes were predicted to lead to improved patient care and higher PICU team and family satisfaction. In order to ensure team-wide, interdisciplinary acceptance and embracement of this project, stakeholders were identified from the PICU physicians, nursing, respiratory therapy staff, and telemedicine administration. Data was tracked for set measures and displayed using run charts. Barriers to implementation were evaluated to improve the process.

The following run charts represent a dashboard of the outcomes being measured. Data was collected via nursing data cards and physician data log books. Of note, the project's three physician leads were the rounding attendings in the early weeks of the project. The physician leads felt they would be best able to role model and support the culture change necessary to make this project successful. This is reflected in the high initial scores for the goals that depend primarily on the leadership of these physicians (technology training, assessing technology challenges and time spent rounding). Overall, all stated goals were achieved and demonstrated sustained improvement. Changes (PDSA cycles) were tested in real-time based on regularly scheduled team meetings and discussions drawing on run chart reviews. PDSAs were also used as an evaluation method in the study of tested improvements and team effectiveness. Sustainability has been achieved and will be maintained by ongoing measurements with intermittent auditing and using a sustainability checklist.

The utilization of telemedicine for inpatient pediatric care is unique within our healthcare system and rare within the national community. This innovation provides expansion of regional PICU coverage in a financially responsible manner while providing improved patient outcomes and improved staff and family satisfaction via immediate access to pediatric intensive care physicians.

Implementation of a telemedicine protocol with consistent daily use allowed the breakdown of initial challenges including staff and parental perception barriers and technological uncertainties while improving the ease of accessibility and staff and parental satisfaction. This technology has led to the
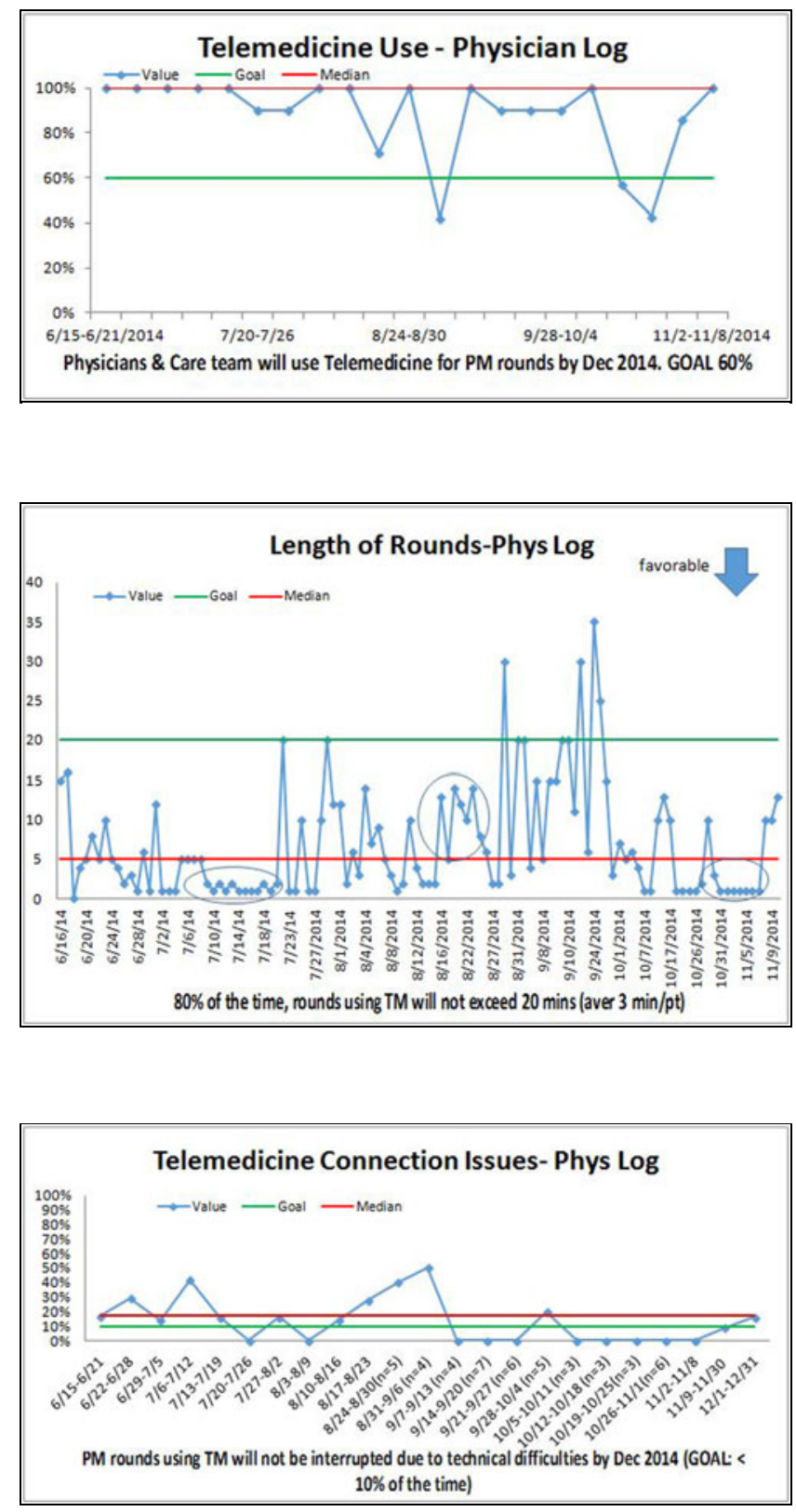


\section{ORAL PRESENTATIONS ABSTRACTS}

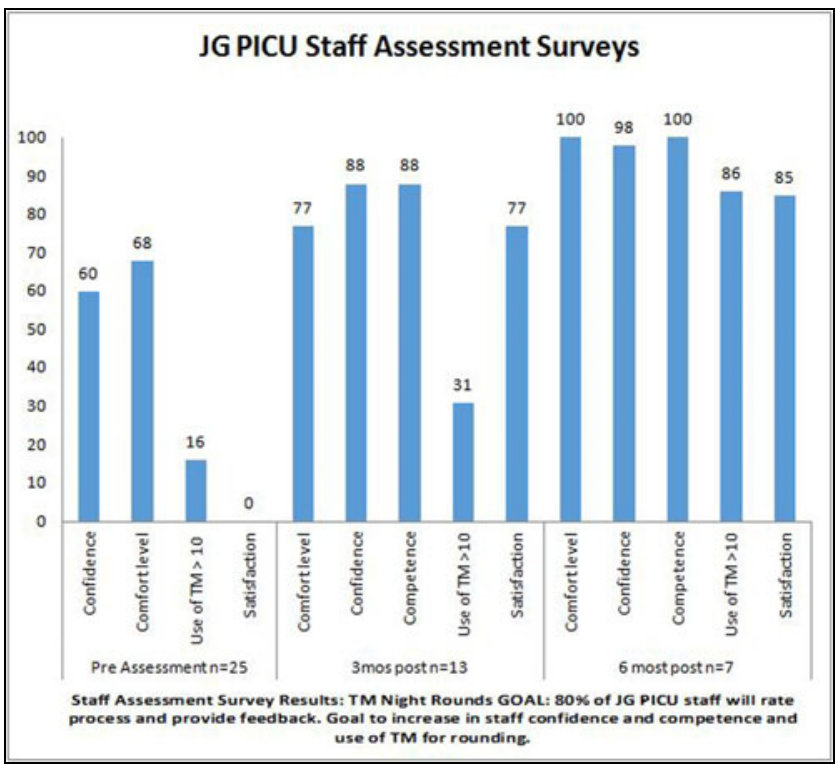

expansion of critical care capabilities and improved the delivery of care as it pertains to the regionalization of PICU coverage in which in-house intensive care physician staffing may not be feasible. Physician utilization increased to 98\% (goal $>80 \%$ ); technology difficulties had a median of $0 \%$ (goal $<10 \%$ ); and staff comfort with technology increased from $28 \%$ to $77 \%$ (goal $>60 \%$ ).

The knowledge gained from the PICU telemedicine rounding project has resulted in a culture change for the rounding model, with behavior alteration of the activities of all care providers within the multidisciplinary team. Additionally, the rounding project improvements are being implemented at other pediatric facilities within our healthcare system.

Future project endeavors will include assessment of a means to optimize medical management plan communication from the medical team to the patients and family, the assessment of staff satisfaction of the newly developed rounding model, and the quantification of cost benefit analysis as it relates to length of stay as well as nursing staff satisfaction and retention in the PICU.

\section{Objectives}

1. Describe the processes utilized in developing a QI framework with PDSA rapid cycle improvement for change to overcome the challenges to develop and staff a regional site Pediatric Intensive Care Unit

2. Describe key project components when planning a regional telemedicine implementation

3. Discuss potential barriers to a successful telemedicine implementation project

HOW TO BUILD A TELEHEALTH PROGRAM IN 18 MONTHS: A PEDIATRIC HEALTH SYSTEM CASE STUDY

PRESENTER: Kristi Moline, MBA, Program Manager

Children's Hospitals and Clinics of Minnesota, Minneapolis, $M N$

Creating a telehealth program can be intimidating and it's difficult to know where to start. From medical records integration to billing and clinical workflow, where does one start on the road to telehealth? In 2014, Children's Hospitals and Clinics of Minnesota (Children's) began its journey to offer virtual services. Eighteen months into the program, with only a Program Manager (0.8 FTE) and Medical Director (0.5 FTE), Children's has made progress toward replicating brick-and-mortar services in a virtual setting including:
- consultations to provide pediatric emergency medicine expertise to outlying hospitals;

- virtual appointments for patients with rare bleeding disorders to consult with a nurse practitioner from the hematology clinic;

- virtual follow-up pulmonary clinic visits

- a pilot program connecting a school nurse with a Children's pediatric nurse practitioner

- a direct-to-consumer program to provide patients with access to Children's virtual care for low acuity illnesses.

The initial progress and successes of the Children's telehealth program has not come without a wide variety of obstacles. This talk will outline significant obstacles and their solutions, important factors contributing to Children's initial success, and key lessons learned as we launched our virtual care programs including:

- The critical need for strong executive support

- The importance of vision casting and how to do it

- The value of strong relationships, both external and internal (legal, credentialing, IT, marketing, communications, Health Information Management, and others)

- Factors allowing movement from concept to implementation

\section{Objectives}

1. Identify key success factors in launching telehealth services

2. Provide knowledge and framework for approaching new virtual care initiatives

3. Discuss how to identify virtual care opportunities and areas of need

\section{SUNDAY, MAY 15, 2016}

1:15 PM-2:15 PM

\section{Sunday, May 15, 2016}

\section{Session 14 Session Title: ROUNDTABLES: PEDIATRIC SPEED ROUNDS}

MODERATOR: Neil E. Herendeen, MD, MS, Associate Professor of Pediatrics

University of Rochester, Pittsford, $N Y$

Connectivity and Technology

PRESENTER: Shawn Farrell, Vice President of Clinical Operations Avizia Inc, Reston, VA

Grant Availability

PRESENTER: Madan Dharmar, MD, MBBS, Assistant Research Professor UC Davis Health System, Sacramento, CA

ICU Care

PRESENTER: Kathleen Webster, MD, MBA, Director of Telemedicine Advocate Children's Hospital, Oak Lawn, IL

PRESENTER: James Marcin, MD, MPH, Professor, Pediatric Critical Care Medicine

UC Davis Health System, Sacramento, CA

Education

PRESENTER: Bryan Burke, MD, Professor, Section of Neonatology University of Arkansas for Medical Sciences, Little Rock, AR

Program Sustainability

PRESENTER: Julie Hall-Barrow, EdD, Senior Director of Healthcare Innovation and Telemedicine

Dallas Children Medical Center, Dallas, TX 


\section{ORAL PRESENTATIONS ABSTRACTS}

School-Based Health

PRESENTER: Steve North, MD, Founder and Medical Director

Center for Rural Health Innovation, Bakersville, NC

PRESENTER: Jimmy McElligott, MD, Medical Director

Center for Telehealth, Medical University of South Carolina, Charleston, SC

Medical Home

PRESENTER: Neil E. Herendeen, MD, MS, Associate Professor of Pediatrics University of Rochester, Pittsford, NY

Entering the field of pediatric telemedicine can be challenging and intimidating for providers interested in starting a new program. This panel will allow participants to choose 3 topics of interest to meet in a small roundtable format with content experts from the Pediatric Special Interest Group. Eight topics will be offered including: Connectivity and Technology, Grant availability, ICU care, Education, Program sustainability, School based health, Medical Home, and International opportunities. Participants are encouraged to bring their questions to the roundtable for immediate answers as well as networking suggestions during the conference. Each of the speakers are published experts in their area of interest and are enthusiastic supporters of new adopters of telemedicine.

\section{REFERENCES}

1. Herendeen N, Deshpande P. Telemedicine and the patient-centered medical home. Pediatr Ann 2014; 43(2):e28-32.

\section{Objectives}

1. Identify key issues to start up a pediatric telemedicine program

2. Identify collaborators and advisors to improve your telemedicine service

3. Network with leaders of the pediatric special interest group

\section{MONDAY, MAY 16, 2016}

8:00 AM-8:45 AM Monday, May 16, 2016

\section{Session 22 \\ Session Title: LESSONS LEARNED ACROSS THREE IN-HOME TELEMEDICINE INITIATIVES SUPPORTING PEDIATRIC PATIENTS AND THEIR CAREGIVERS}

MODERATOR: Janine Gracy, MSE, CHES, CPP, Project Director

KU Center for Telemedicine \& Telehealth, Fairway, KS

PRESENTER: Susana Patton, PhD, CDE, Associate Professor

University of Kansas Medical Center, Kansas City, KS

PRESENTER: E. LaVerne Manos, DNP, RN-BC, Program Director University of Kansas, Master of Science in Health Informatics Program, Kansas City, KS

PRESENTER: Eve-Lynn Nelson, PhD, Director KU Center for Telemedicine \& Telehealth, Fairway, KS

Introduction: Pediatric telemedicine has a growing evidence base in supervised settings. There is strong consumer and provider interest in extending pediatric services to unsupervised settings such as the home. Home-based telehealth services have advantages including convenience, decreased stigma, increased family participation, and increased generalizability of skills practices in the child's lived environment. While only a handful of in-home pediatric telehealth trials have been reported to date, studies are very much needed to inform benefits, risks, and family-centered best practices. The panelists will present research from three in-home telehealth projects supported through the Uni- versity of Kansas Center for Telemedicine \& Telehealth. The projects utilize tablets using a secure cloud-based videoconferencing solution in order to reach families in metropolitan as well as rural/remote areas.

Methods \& Results: The first panelist (Dr. Patton) will summarize a NICHDfunded project that tests the feasibility, acceptability, and initial efficacy of a real-time videoconferencing intervention addressing parents' fear of hypoglycemia and parenting stress in families of young children with type 1 diabetes (T1DM). By combining T1DM education, behavioral-parent training, and cognitive-behavior therapy, the project aims to reduce fear of hypoglycemia and stress in parents and improve health outcomes for young children. The presenter will summarize adapting an intervention protocol for the telehealth setting. Overall, 48 parents, recruited in 3 cohorts, are being randomly assigned to either the immediate treatment or wait-list control conditions to allow for iterative treatment refinement. The in-home televideo intervention includes 10-sessions alternating between group and individual meetings. The presenter will describe findings from the first cohort across parents' fear of hypoglycemia stress, children's HbA1c, and children's glycemic variability. The second panelist (Dr. Manos) leads the HRSA-funded QI- approach, Interprofessional Collaborative Acute-Care Practice-Pediatric Project. Technologies support training the pediatric care teams in TeamSTEPPS communication strategies in order to promote family-centered care and enhance care coordination. The telehealth follow-up component utilizes tablet technology to connect the inpatient team with the family within 24-48 hours post-discharge. Across the first 80 encounters (average 2 encounters/family), the most common presentations were asthma, dermatology, cystic fibrosis, and surgery follow-up. Some patients were discharged earlier due to the ability to follow complex healthcare conditions and newly diagnosed conditions more closely. It bridges the gap between the first outpatient follow-up. The televideo connection enhanced team-family communication around adherence and care coordination. Patient engagement was rated well both pre $(n=70, M=16.16, S D=8.42)$ and post $(n=41, M=16.56, S D=6.17)$ project implementation with no significant differences (Scotten, Manos, Malicoat, \& Paola, 2015), which may reflect a ceiling effect. The third panelist (Dr. Nelson) will present pediatric findings from the NIBIB-funded Mobile Technologies Assisting Patients and Family Caregivers in Healthy Living project (PI: Dr. Carol Smith, R01EB015911). Patients receiving home parenteral nutrition (HPN), a life-sustaining intravenous (IV) infusion that provides nourishment and hydration to patients with short gut or inflammatory bowel diseases, are often isolated and overwhelmed. Patients and family caregivers often experience difficulties with fatigue, depressive disorders, loss of sleep and worry over the potential life-threatening side effects and the expenses. The randomized clinical trial evaluated a mobile videoconferencing-delivered support group intervention for patients on HPN and their caregivers, connecting multiple families to trained facilitators in real-time. Of 126 enrolled in the randomized trial, 111 were adults and 15 were teens. Pediatric-specific lessons will be emphasized across positive outcomes related to feasibility (Smith et al., in press), cost (Kim et al., 2014), quality of life/“achieving normalcy” (Winkler \& Smith, 2015), and support for mental health concerns (Nelson et al., under review).

Conclusion: The three research projects support feasibility and initial positive outcomes providing pediatric telehealth services to the home. The secure, low-cost technology solution offers an appealing way to increase access to support families, including parents of young children with diabetes, children post-discharge from inpatient settings, and teens on home parenteral nutrition and their caregivers. Next research steps will be discussed, including plans for mixed methods, multi-site trials. The panelists will conclude by summarizing efforts to disseminate findings to inform clinicians as well as decision makers around policy and reimbursement.

\section{REFERENCES}

1. Smith CE, Werkowitch M, Piamjariyakul U, Yadrich D, Thompson N, Fitzpatrick S, Kim H, Nelson E. Translation of IV Home Care Interventions into Practice via Mobile Distance Delivery. Comput Inform Nurs, In Press. 
2. Nelson $E$, Werkowitch $M$, Piamjariyakul U, Yadrich D, Thompson N, Smith CE. (Under review). The Evaluation of Depressive Symptoms in Mobile Videoconferencing-Based Support Group Sessions for Home Parenteral Nutrition (HPN) Users and Their Family Caregivers. Telemedicine J E Health.

3. Scotten M, Manos EL, Malicoat A, \& Paolo AM. Minding the gap: Interprofessional communication during inpatient and post discharge chasm care. Patient Educ Couns 2015; 98(7):895-900.

4. Patton SR, Odar C, Midyett LK, Clements MA. Pilot study results for a novel behavior plus nutrition intervention for caregivers of young children with type 1 diabetes. J Nutr Educ Behav 2014; 46(5):429-33.

\section{Objectives}

1. Summarize implementation lessons from three in-home pediatric telehealth research projects

2. Explain results from across the three pediatric telehealth studies

3. Describe next research steps building upon the three studies

\section{MONDAY, MAY 16, 2016}

11:00 AM-12:00 PM

Monday, May 16, 2016

\section{Session 30 Session Title: URGENT CARE AT SCHOOL OR MUCH MORE?}

MODERATOR: Steve North, MD, Founder and Medical Director Center for Rural Health Innovation, Bakersville, NC

PRESENTER: Kelli Garber, MSN, APRN, PPCNP-BC, School-Based Telehealth Nurse Practitioner

Medical University of South Carolina, Charleston, SC

PRESENTER: Amanda Martin, BA, Executive Director Center for Rural Health Innovation, Spruce Pine, NC

PRESENTER: Elana Wells, MPH, CHES, Program Manager, School-Based Telehealth

Medical University of South Carolina, Charleston, SC

Introduction: Long travel distances, missed work days for parents, and missed school days are barriers to accessing healthcare for many rural or underserved children. School-based Telehealth (tSBHC) is an innovative and effective way to improve access to acute sick care, chronic disease management, and mental health services by bringing the care to children right where they are, in school. With many for profit companies seeking to add schools to the growing list of direct to consumer telehealth sites, it is important to understand how a true School-Based Telehealth program is different. School-Based Telehealth can also be an important part of an Accountable Care Organization (ACO) contributing to the goal of providing quality care with cost savings.

Methods: Challenges exist to developing a successful tSBHC program. Each school, community, culture and delivery system may require a different approach. As school-based telehealth has expanded, a variety of models have developed to fit the needs of the community. Experts from two successful school-based telehealth programs will participate in this panel discussion exploring various models of school-based telehealth. A discussion of each program's scope of care, structure, workflow, funding, support, and services offered will highlight the variety of ways telehealth can be provided in schools. Key methods to the success of a program, including how to integrate the program into the local culture, as well as lessons learned will be shared.

Results: A successful tSBHC program includes integration with the culture of the school, the local community, and local healthcare providers. It will seek to compliment the medical home rather than to replace it. The goal is not one of financial gain, rather of improving access to quality healthcare.
Conclusion: The value of tSBHC care lies in its ability to expand access to quality healthcare to all children while preserving the medical home. This presentation will provide an overview for the various models of tSBHC, methods for integrating the program into the local community, and share how the various program models offer more comprehensive care than the emerging direct to consumer trend.

\section{REFERENCES}

1. Council on School Health. School-based health centers and pediatric practice. Pediatrics 2012; 129(2):387-93.

2. North SW, McElligot J, Douglas G, Martin A. Improving access to care through the patient-centered medical home. Pediatr Ann 2014; 43(2):e33-38.

3. Reynolds CA, Maughan ED. Telehealth in the school setting: an integrative review. J Sch Nurs 2015; 31(1):44-53.

\section{Objectives}

1. Increase knowledge in the various program models of school-based telehealth

2. Demonstrate awareness regarding the difference between school-based telehealth and urgent care

3. Increase knowledge for how to integrate a program into community's culture

\section{MONDAY, MAY 16, 2016}

1:15 PM-2:15 PM Monday, May 16, 2016

\section{Session 36 Session Title: RESEARCH RESULTS FOR PEDIATRIC PROGRAMS}

MODERATOR: S. David McSwain, MD, MPH, Medical Director, Inpatient and Emergency Teleconsultation

MUSC Center for Telehealth, Associate Professor, Pediatric Critical Care Medicine

MUSC Children's Hospital, Charleston, SC

PEDIATRIC TELEHEALTH PROGRAMS IN THE UNITED STATES: GROWING AND DIVERSIFYING

PRESENTER: James T. McElligott, MD, MSCR, Medical Director for Telehealth ${ }^{1}$, Kelli Garber, MSN, APRN, PPCNP-BC ${ }^{2}$,

Elana Wells, MPH, CHES ${ }^{2}$

${ }^{1}$ The Medical University of South Carolina, Charleston, SC, ${ }^{2}$ MUSC, Charleston, SC

Introduction: The use of telehealth has grown rapidly in recent years, with a 400\% increase in its use since 2012 to estimated 100 million e-visits performed in 2014. Pediatric services that utilize telehealth are likely to be growing, but no assessment of growth or variation in focus of these services has been done to date. Methods: The Pediatric Special Interest Group (Peds SIG) of the American Telemedicine Association conducted a Pediatric Telehealth Census in 2011 to determine the number and diversity of various pediatric telehealth programs across the country. In 2011, the census was compiled through a survey of the members of the American Telemedicine Association (ATA) Pediatric Special Interest Group. Each respondent indicated whether the institution for which they work offers pediatric telemedicine services for each of the Accreditation Council for Graduate Medical Education (ACGME) specialties for inpatients as well as for outpatients. The same method was used to evaluate the diversity of international pediatric telemedicine services. The survey was repeated in 2015 and expanded to include the members of the American Academy of Pediatrics' (AAP) Section on Telehealth Care. 


\section{ORAL PRESENTATIONS ABSTRACTS}

Results: The number of responding organizations grew from 22 in 2011 to 48 in 2015. The findings for inpatient specialties indicated a $24 \%$ increase in the number of specialties offering pediatric telehealth services (from 25 to 31 programs). The diversity of outpatient pediatric programs grew even more significantly with a 61\% increase (from 23-37 programs). The most notable increase was seen in the international pediatric telemedicine services being offered as evidenced by growth of 380\% (from 5 to 24 programs). The census also revealed a number of unique pediatric telemedicine programs. The inpatient unique programs identified were: hospice and palliative medicine, plastic surgery (including craniofacial surgery), and thoracic surgery (including congenital cardiac surgery). The only unique outpatient program was that of pain management. The largest growth in unique programs was in international telemedicine. The programs identified were burn surgery, general pediatrics, infectious disease, nephrology, neonatal-perinatal medicine, nuclear medicine, ophthalmology, pathology, rheumatology, and congenital cardiac surgery.

Conclusions: The increase in total number of pediatric organizations utilizing telehealth as well as a greater number of services offered by each organization indicates that the use of telehealth in pediatrics is following the national trend of growth. In fact, several services in the census identified 10 or more existing telehealth programs including inpatient critical care, outpatient psychiatry, general pediatrics, and endocrinology and interpreting services. The development of common services across multiple institutions provides an opportunity for the development of pediatric telemedicine subgroups. By partnering with one another through the implementation of specialty specific subgroups, each telemedicine program will benefit by the experience and expertise of other programs providing similar services. This will not only improve the quality of care being provided but also allow for the development of guidelines and standards. The lack of existing evidence within the pediatric telehealth field could also be addressed by the development of pediatric specialty specific subgroups. By establishing a common set of measures and combining data across each subgroup, outcomes will be stronger and more generalizable.

\section{Objectives}

1. Describe the change in growth and diversity of pediatric telehealth services since 2011

2. Identify the types of pediatric services being provided via telehealth

3. Analyze the benefits of developing pediatric specialty specific subgroups for telehealth

THE EFFECT OF APRN TELEHEALTH CARE COORDINATION ON MISSED SCHOOL AND WORK DAYS FOR CHILDREN WITH COMPLEX MEDICAL CONDITIONS AND THEIR CAREGIVER

PRESENTER: Stanley M. Finkelstein, PhD, Professor ${ }^{1}$, Rhonda Cady, $\mathrm{PhD}^{2}$, Wendy Looman, $\mathrm{PhD}^{1}$

${ }^{1}$ University of Minnesota, Minneapolis, $M N,{ }^{2}$ Gillette Children's Specialty

Health Care, St. Paul, MN

Introduction: This observational study was part of the TeleFamilies Research Study (NIH R01- NR01883) to investigate the effectiveness of advanced practice registered nurse telehealth care coordination for children with complex medical conditions (CMC). One of the effectiveness measures was the number of school/day-care days missed by the child with CMC and work missed by the child's caregiver as a result of the TeleFamilies care coordination intervention. TeleFamilies was a three armed randomized controlled trial using usual LPN/RN delivered care coordination and telephone triage (control group), or advanced practice registered nurse (APRN) delivered care coordination via telephone (telephone group), or APRN delivered care coordination using telephone plus video (video group) communications with families at home. Caregivers (parents/guardians) of participating families were asked to complete a Health Care Services Utilization (HCSU) calendar each month during a six month baseline data collection period and the two year ran- domized trial intervention period. The HCSU calendar was used to track information including hospital and clinic visits and whether the child missed school/day-care and the caregiver missed work during each reported visit. This observational study compared the number of reported missed school and work days during baseline and the RCT intervention periods for subjects in each of the three study arms.

Methods: TeleFamilies was based in a large urban pediatric primary care clinic affiliated with a non-profit children's hospital. Children recruited for TeleFamilies were between 2 and 15 years of age, from English speaking households, and met at least four of five criteria in the Children with Special Health Care Needs Screener. There were 163 subjects consented for TeleFamilies following Institutional Review Board guidelines. Subjects were randomized into one of the three study arms. Of these, 149 subjects completed the 30- month data collection period ( 6 month baseline and 2 year intervention). Some subjects did not complete the study due to death, moving out of state, or situational barriers. The HCSU data from the 149 subjects who completed the study were analyzed. All 149 subjects were considered as a single group during baseline data collection. During the RCT intervention these subjects constituted the control group (47 subjects), the telephone group (50 subjects) and video group (52 subjects). The electronic medical record (EMR) was used to obtain utilization data for subjects who did not provide HCSU self reports. The missed school/work information reported on the HCSU form was not available in the EMR. Visit reports based solely on EMR review were not included in this missed school/work analysis. Clinical visits outside the study institution were not available in the EMR.

Results: There were 505 visits that resulted in missed school during the 6 month baseline and 2082 during the 24 month intervention periods. There were 304 reports of missed work time during baseline and 1063 during the intervention period. Based on the number of subjects, there were 0.56 days of missed school/subject/month and 0.34 days of missed work-time/month for caregivers during the baseline period. During the intervention period these values changed to 0.58 missed school days/subject/month for the overall study population, broken down to 0.46 missed school for the control group, 0.59 for the telephone group and 0.68 for the video group. Missed work time per month also changed to 0.30 for the overall group during the intervention period, broken down to 0.28 for the control group, 0.32 for the telephone group and 0.30 for the video group. Conclusion: The monthly per subject missed school increased while missed work decreased from baseline to intervention, but these directional changes were not consistent between the three study groups during the intervention. The control group showed fewer missed school and work during the intervention compared to baseline periods, and less missed school and work than the intervention groups during the intervention period. The increase in missed time for the intervention groups may be due to the closer surveillance and intervention as a result of the APRN training and scope of practice, or due to increased missing HCSU self-reports from the control group during the intervention period. This reduced self-reporting among controls could be a result of fewer interactions between subject caregivers and the control triage nurses than the interactions between caregivers and the intervention APRN. Relationship building was an important part of the APRN care coordination effort and went beyond the usual nurse triage practice, and may have resulted in better HCSU calendar return rates. Finally, school/work information is not a part of the institution EMR, so that reliance on EMR for missing HCSU selfreports will overlook the missing school/work issues related to clinic visits.

\section{REFERENCES}

1. Looman W, Presler E, Erickson M, Garwick A, Cady R, Finkelstein S. Care coordination for children with complex special healthcare needs: The value of the advanced practice nurses enhanced scope of knowledge and practice. $J$ Pediatric Health Care 2013: 27(4):293-303.

\section{Objectives}

1. Apply telehealth supported care coordination for children with complex medical conditions 
2. Learn how care coordination by advanced practice registered nurses and LPN/RN differ

3. Learn about impact of telehealth care coordination on missing school and work for child and caregiver, respectively

\section{FAST AND FURIOUS 2: THE DEVELOPMENT AND EXPANSION OF THE FASTEST GROWING SCHOOL-BASED TELEHEALTH PROGRAM IN THE COUNTRY: A CASE STUDY}

PRESENTER: Tamara Perry, BS, Director of Telehealth Operations Children's Health System of Texas, Dallas, TX

Access to primary care for children who are Medicaid eligible in North Texas is limited due to lack of providers accepting Medicaid, non-enrollment in Medicaid and limited or no pediatric providers in geographic locations. With funding from the Texas Waiver Project - Children's Health System of Texas launched a school-based telehealth program that provides acute primary care services in 26 schools in the Summer of 2014. By the Fall of 2015, the program expanded to 58 schools and has a projected expansion to add 26 more schools by the end of school year 2015-2016. This deployment includes both Independent School Districts and Charter Schools located in urban and rural locations.

Utilizing emergency room data usage and pediatric coverage data - potential schools administrators were contacted for participation. This presentation will provide the details of implementing school telehealth in eleven different school entities for a total of 84 schools including: Work plans, Clinical Work-flow, Infrastructure and deployment, Training and Education of Nurse Presenters, Regulatory hurdles, Registration and Access, On-going monitoring and assessment, Best Practices and Lessons Learned.

The program's objective is to improve access to primary care for North Texas students, to reduce emergency room visits for non-emergent conditions, decrease absenteeism for health related reasons and assess patient/family satisfaction with telehealth. Deploying telehealth in schools on a large-scale is achievable but not without lessons learned along the way. As the school-based telehealth program expands in 2015-2016; many of the operational and implementation lessons will be included in the operation and implementation plan.

All information is the intellectual work of the Children's Health System of Texas Telehealth Team.

\section{Objectives}

1. Apply valuable techniques in the development and implementation of school based telehealth

2. Identify tools to the operational development of a diverse school based telehealth program

3. Develop a plan of action for the development of their own school based telehealth program

\section{MONDAY, MAY 16, 2016}

4:15 PM-5:15 PM

Monday, May 16, 2016

\section{Session 43 \\ Session Title: TELEMENTORING FOR COMMON AND COMPLEX DISEASE MANAGEMENT}

MODERATOR: Sucheta Joshi, MD, MS, FAAP, Clinical Associate Professor University of Michigan, Ann Arbor, MI

PRESENTER: Trisha Calabrese, MPH, Director, Division of Innovation American Academy of Pediatrics, Elk Grove Village, IL

PRESENTER: Jeremy Toler, MD, Physician, Pediatric Neurology. Children's Hospital Colorado, Aurora, CO
It is estimated that 32 million Americans, including a large number of children, will become newly insured in the next decade (1). Although children and youth are one of the fastest growing populations and suffer from chronic conditions such as epilepsy, many do not have access to pediatric specialists for high-quality coordinated care provided in a medical home, especially in rural and medically underserved areas. This is further exacerbated by a national shortage of pediatric subspecialists and primary care providers who treat common and complex diseases. On average, there are 100,000 to 200,000 children per pediatric specialty provider in the U.S. (2), and there are only 28,000 pediatric subspecialists and surgical specialists to care or over 80 million children (3). As a result of increased demand for pediatric subspecialty care, many families have to travel several hours from rural areas or endure lengthy waiting times for clinic appointments.

To help remediate appointment wait times, increase access to pediatric subspecialty care and develop quality improvement strategies with a mobilized community of learners, the American Academy of Pediatrics (AAP)'s Coordinating Center for Access and Services for Children and Youth with Epilepsy (CYE) has adapted the national Project ECHO (Extension for Community Healthcare Outcomes) model for CYE. As a step toward this goal, the program is assessing the feasibility of the ECHO model for CYE across diverse regions of the country- New Mexico, Colorado, Illinois, Kansas, Missouri, and New York. It is estimated that one in 26 individuals will develop epilepsy in their lifetime. Epilepsy is one of the most common and complex neurologic conditions in the United States among children and serves as an ideal paradigm for care coordination of children with complex health needs, including multiple co-morbidities. The ECHO model links expert specialist teams at the academic 'hub' with primary care clinicians in local communities 'spokes' using state-of-the-art video-conferencing technology. This partnership helps patients get the right care, in the right place, at the right time, standardize treatment and referral patterns and promote medical home goals. Ultimately, the goal is to de-monopolize knowledge and increase workforce capacity to provide best-practice specialty care and reduce health disparities. Collaboration between specialists and community providers enables patients to receive care from the professionals they know and trust in their own communities. For providers, co-management advances knowledge and feelings of confidence in managing complex cases, as well as decreases professional isolation. Together, they manage patient cases so that patients get the care they need rapidly.

As the program is disseminated, the long-term outcome is for healthcare providers to increase knowledge, competency and self-efficacy in the management of pediatric epilepsy, which in turn, enables patients to receive culturally competent care from local primary care providers, resulting in better quality and great access to healthcare. To-date, preliminary baseline findings indicate that family members feel uncomfortable about medication management, knowing the basics about seizures and how to support their child's mental or emotional health. Moreover, specialists and non-specialists have low levels of confidence and knowledge when providing different levels of care for CYE.

As healthcare moves towards a population health approach and physician practices transform, it is essential that we build a patient-centered medical home incorporating telementoring to address children with any complex, medical conditions within the quality framework and empower care providers. This presentation will describe an innovative health delivery model, Project ECHO, discuss outcome evaluation assessing increase in awareness and access to care and changes knowledge and self-efficacy among family members and PCPs with regards to managing and treating CYE and discuss the benefits of telementoring to extend the continuum of remote care and create a community of learners to build capacity among all primary care physicians (PCPs) to treat other common and complex health conditions.

\section{REFERENCES}

1. Association of American Medical Colleges. Physician Shortages to Worsen without Increases in Residency Training. Retrieved September 11, 2015, from 
https://kaiserhealthnews.files.wordpress.com/2014/04/physician_shortages_ to_worsen_without_increases_in_residency_tr.pdf

2. Mayer ML. Are we there yet? Distance to care and relative supply among pediatric medical subspecialties. Pediatrics 2006; 118(6):2313-321.

3. American Academy of Pediatrics. America's Children Need Access to Pediatric Subspecialists. Retrieved from https://www.aap.org/en-us/about-the-aap/ departments-and-divisions/department-of-education/Documents/ Sec5203FactSheet.pdf

\section{Objectives}

1. Recognize how innovation builds capacity among primary care providers while increasing access to subspecialty care

2. Understand how Project ECHO increases access to quality care resulting in improved outcomes for children and youth with chronic health conditions

3. Envision how this model of care can influence other changes in the delivery of healthcare

\section{TUESDAY, MAY 17, 2016}

\section{1:00 AM-12:00 PM Tuesday, May 17, 2016}

\section{Session 51}

\section{Session Title: UNDERSTANDING OUTCOME MEASURES AND EVALUATION METHODS IN PEDIATRIC TELEHEALTH}

MODERATOR: Madan Dharmar, MBBS, PhD, Assistant Research Professor UC Davis Children's Hospital, Sacramento, CA

PRESENTER: Kathleen Webster, MD, MBA, Director of Telemedicine Advocate Children's Hospital, Oak Lawn, IL

PRESENTER: Neil E. Herendeen, MD, Associate Professor of Pediatrics University of Rochester, Pittsford, NY

PRESENTER: Madan Dharmar, MBBS, PhD, Assistant Research Professor UC Davis Children's Hospital, Sacramento, CA

Introduction: In assessing the impact of telemedicine programs, it is important to keep in mind how to determine if established goals have been met. The goals of telemedicine programs are often cited as the need to improve the quality of, access to, and efficiency of care in a financially sustainable manner. In designing a program, it is important to consider how these goals will be evaluated.

Methods: This panel will discuss various types of outcome measures that can be evaluated in a tele-health program based on the programmatic and institutional needs. The panel will explore explicit outcome measures such as medication error rates, transfer rates and criterion-based outcomes; implicit measures such as quality and satisfaction of care; utilization measures such as cost-effectiveness, emergency department utilization and health dollars spent per patient. To achieve our objectives, the panel will illustrate the why, who, how and what defines possible outcome measures by discussing the experiences of researchers in developing an evaluation plan for their program. This panel will discuss the following successful telemedicine programs: (1) Internal telemedicine programs, designed to meet quality and patient safety goals needs within a health system; (2) Pediatric Telemedicine program, which has shown higher quality and satisfaction of care, improved patient safety, and cost effective delivery of pediatric specialist to rural/underserved hospitals; and (3) Health-e-Access program which has shown a decrease in emergency department utilization and healthcare dollars spent by providing access to daytime pediatric telemedicine services to school and childcare centers.

Conclusion: The use of telemedicine keeps increasing exponentially and continues to change the way healthcare is delivered. Through this panel, we will help enable providers to establish evaluative outcome measures which will assess the success of their telehealth program.

\section{Objectives}

1. Discuss the importance of measurable outcomes in evaluating and improving tele-health programs which deliver telemedicine services to care for children

2. Discuss the why, who, how and what defines possible outcome measures for tele-health program evaluation

3. Discuss cost/utilization outcome measures

\section{TUESDAY, MAY 17, 2016}

1:15 PM-2:15 PM

Tuesday, May 17, 2016

\section{Session 59 \\ Session Title: USING TELEHEALTH TO SUPPORT PEDIATRIC PATIENTS IN CRITICAL SITUATIONS IN THE U.S. AND ABROAD}

MODERATOR: Mark Waltzman, MD, Chief of Pediatrics

South Shore Hospital, Weymouth, MA

PRESENTER: Kathleen Kramer, MAT, BS, PMP, RT (R), Consultant Cincinnati Children's Hospital Medical Center, Cincinnati, $\mathrm{OH}$

PRESENTER: Shayan Vyas, MD, Medical Director

of Nemours Florida Telemedicine

Nemours Children's Hospital, Orlando, FL

PRESENTER: Alejandro J. Lopez-Magallon, Assistant Professor, Critical Care Medicine

Cardiac ICU/Telemedicine/eCICU, Children's Hospital of Pittsburgh, Pittsburgh, PA

Telemedicine technology enables pediatric hospitals across the nation to provide timely access to high quality care, with the goal of improving health outcomes and the patient and family experience. In this moderated panel, four children's hospitals will describe successful implementations of telehealth programs focused on delivery of acute pediatric care from a distance. The TeleConnect Program at Boston Children's Hospital (BCH) uses real-time interactive video to support on-demand clinical collaboration between BCH's critical care intensivists and community emergency department (ED) physicians in the evaluation and stabilization of acute pediatric patients. Cincinnati Children's Hospital Medical Center (CCHMC) integrated telehealth technology into a new 42-bed inpatient unit located 25 miles north of the main medical campus to support Code and Medical Response Teams and critical care rounding. Nemours Children's Health System has developed a telemonitoring program in which telehealth technology is used to remotely monitor 150 patient beds including PICU beds in Delaware from the Florida campus. Finally, Children's Hospital of Pittsburgh of UPMC partners with hospitals in South America to provide critical care services to pediatric patients, including rounding and specialty consultations. This panel presentation will demonstrate how telehealth can expedite access to high quality pediatric specialty care in the community hospital setting in the United States and abroad to support collaborative clinical decision making in critical situations, leading to improved health outcomes and better patient and family experiences. Presenters will discuss the importance of customizable training, simulation, and involvement of key stakeholders, families, and patients in the development of these programs.

Objectives

1. Summarize how children's hospitals are using telehealth to provide access to critical care services

2. Compare models for the use of telehealth in the United States and abroad in meeting the healthcare needs of pediatric patients in critical situations

3. Describe best practices that can be used to implement telehealth programs to improve access to pediatric critical care services 


\section{TUESDAY, MAY 17, 2016}

3:00 PM-4:00 PM

Tuesday, May 17, 2016

\section{Session 67 \\ Session Title: PILOTING A TELEHEALTH GROUP WITH ADOLESCENTS: LESSONS LEARNED FROM A PEDIATRIC TRANSPLANT GROUP}

PRESENTER: Sarah L. Kelly, PsyD, Clinical Psychologist

Children's Hospital Colorado, Aurora, CO

PRESENTER: Cindy Buchanan, PhD, Clinical Psychologist

University of Colorado School of Medicine, Aurora, CO

Solid organ transplantation is a life-saving treatment in many end-stage diseases. However, it is a chronic illness requiring strict adherence to a complex post-transplant medical regimen. Poor medication adherence is associated with serious and potentially fatal consequences and adolescents are most at-risk for nonadherence. Unlike other post-transplant complications, adherence can be directly modifiable. Individual, family, and group interventions can be effective in improving adherence in adolescents. At our pediatric transplant center, families and providers have identified a need for adolescent group treatment; however, many teens are not able to attend groups as the majority of our families reside greater than 100 miles away.

Behavioral health provider shortages, barriers to care, and rapidly evolving changes in the healthcare landscape drive the implementation of telehealth services. Natural settings, such as the patient's home or school, are optimal settings for telehealth (Grady, Myers, Nelson, Belz, Bennett et al., 2011) and youth are well suited for interactive videoconferencing. Hommel, Hente, Herzer, Ingerski, and Denson (2013) have described family interventions to improve adolescent adherence via videoconferencing and found this medium to be feasible and acceptable to patients and caregivers. Studies examining videoconferencing groups are extremely limited, but it is a promising tool in behavioral health with comparable satisfaction, retention of information, attendance, attrition, group cohesion, and effectiveness, especially for patients who struggle with an isolating condition (Grady et al., 2011; McGrath Davis, Sampilo, Gallagher, Landrum, \& Malone, 2013). However, to date there are no telehealth interventions focused specifically on adolescent solid organ transplant recipients and no videoconferencing groups have been conducted with youth.

We designed a five-session videoconferencing group targeted at improving adherence in teens aged 13-17 who have received a heart, kidney, or liver transplant. This telehealth group provides peer support, education, behavioral modification, problem-solving strategies, and goal-setting to address family and peer issues related to transplant and to improve medication adherence. Pre- and post-intervention assessments track changes in factors such as adherence, hope, health-related quality of life, mood, and family environment. Prior to the first intervention group, each adolescent participated in an individual technology orientation session. After the group, a mixed quantitative and qualitative Telehealth Group Satisfaction Survey was administered to assess participants' satisfaction with the group and with the telehealth technology.

The telehealth groups have begun and we continue to enroll adolescents. This tutorial presentation will describe results in ways that illustrate practical suggestions and strategies for implementation of telehealth groups with youth. Early analyses suggest that this group is acceptable to adolescent transplant recipients, though they experienced occasional technical difficulties. Preliminary open-ended responses from the adolescents highlight strengths of the group and offer suggestions for how to improve the patient experience. For example, one participant stated, "I liked how we could all relate to each other and speak openly with one another about our experiences and learn from one another," and described, "logging in was easy but staying connected for the full hour was the hard part because sometimes the WiFi connection wasn't working very well." The facilitator also documented obstacles to implementation. Challenges to feasibility that required further problem-solving included patients being hospitalized during the group, patients with slow Internet connections, and having to end a group prematurely due to technological issues. We learned, for example, that it is important to include parental oversight in scheduling the group and provide frequent reminders to help youth remember to log in for each group. Additionally, we found that though we recommended using a computer or tablet for the group, many youth utilized a mobile phone due to lack of access to a larger device despite some limitations associated with mobile participation.

Our experience facilitating telehealth groups with adolescents provides insights into strategies to optimize feasibility, the patient experience, and effectiveness of the intervention as delivered via a videoconferencing platform. We will explore and discuss the challenges to implementing a home-based group, suggest practical strategies and adjustments when working with adolescents, and help the audience to anticipate obstacles to implementing a telehealth group with youth. Implementation of groups such as these have the potential to expand services and improve outcomes for youth and they provide one innovative solution to barriers to quality care.

\section{REFERENCES}

1. Grady B, Myers KM, Nelson EL, Belz N, Bennett L, Carnahan L, et al. Evidencebased practice for telemental health. Telemed and E Health 2011; 17(2):131-48.

2. Hommel KA, Hente $E_{1}$ Herzer M, Ingerski LM, Denson LA. Telehealth behavioral treatment for medication nonadherence: A pilot and feasibility study. Eur J Gastroenterol Hepatol 2013; 25(4):469-73.

3. McGrath Davis A, Sampilo M, Gallagher KS, Landrum Y, Malone B. Treating rural pediatric obesity through telemedicine: Outcomes from a small randomized controlled trial. J Pediatr Psychol 2013; 38(9):932-43.

\section{Objectives}

1. Demonstrate awareness of the current state of the literature for conducting group telehealth interventions with youth

2. Identify practical strategies for implementing a telehealth group with pediatric patients

3. Apply knowledge to anticipate obstacles when utilizing home-based telehealth with a group of adolescents

\section{TUESDAY, MAY 17, 2016}

4:15 PM-5:15 PM Tuesday, May 17, 2016

\section{Session 75}

\section{Session Title: CREATIVE SOLUTIONS TO SCHOOL NURSING NEEDS}

MODERATOR: Julie Donner, MSSL, BS OT, Quality and Innovation Manager

Avera eCARE, Sioux Falls, SD

PRESENTER: Molly Satter, RN, MSN, Health Services Supervisor Sioux Falls School District, Sioux Falls, SD

PRESENTER: Linda J. Young, RN, MSN, FRE, BC, Nursing Program Specialist

South Dakota Board of Nursing, Pierre, SD

PRESENTER: Mary Oyos, RN, MS, BC-ADM, CDE, Diabetes Program Manager

Avera McKennan, Sioux Falls, SD

Students in rural school systems who have chronic health conditions face challenges in their ability to access care during their school day due to limitations in school nurse services. Through an innovative and collaborative Virtual Nurse Pilot (Young and Damgaard, 2014), an evidence base investigation lead to public policy changes that allows supervised and trained unlicensed assisted personal (UAP) to provide certain diabetic care in the schools (Young and Damgaard, 2015). Technology to allow virtual supervision is an 


\section{ORAL PRESENTATIONS ABSTRACTS}

important part of this change. These changes have inspired additional applications of technology and lead to additional exploration.

Another eCARE School Nurse pilot which is providing school nurse coverage for urgent care needs to UAPs in schools systems. The pilot began with six urban elementary schools which had in house nurse coverage for student needs only part days each week. Through this program, UAPs who want a nurse consultation can contact a dedicated nurse at all times during the school day using technology. UAPs are trained to present student health needs over video with use of peripheral devices. This pilot is now beginning its second school year, and looking to expand the care model from a larger school system to a rural school system where face to face school nurse time is reserved for routine screenings, and the administration of school health required activities only. Providing the UAPs which are in many cases school clerical staff a way to get a nurse's opinion for unplanned student health concerns has been a big satisfier of school staff. Early data shows that virtual school nurses were consulted in 5\% of student health requests, and 84\% of the cases were seen and returned to class. Keeping students well, ready to learn and in school are primary objectives of all school health interventions and programs. Additional lessons learned during the initial months were the importance of relationship building and equipment ease as factors in the success of this care model. There are continued barriers to the application of this model and the expansion of this model. Those include sustainability, staffing challenges for peak times where students need care, and the appropriate balance of expertise in nursing care to provide urgent nurse consultations as well as the supervision of chronic disease management as in the case of diabetes care discussed.

\section{REFERENCES}

1. South Dakota's Administrative Nurse Delegation Rules (a) 20:48:04.01:09. Registration required for delegated medication administration and (b) 20:48:04.01:11. Medication administration tasks that may not be routinely delegated and require written protocol.

2. ARSD 20:48:04.01:16. Written protocol required for the delegation of insulin administration by the subcutaneous route to unlicensed assistive personnel.

3. Young L, Damgaard G. Virtual Nursing Care for School Children with Diabetes. J Nurs Regul 2014; 4(4):15-24

4. Young L, Damgaard G. Transitioning the Virtual Nursing Care for School Children With Diabetes Study to a Sustainable Model of Nursing Care. J Nurs Regul 2015; 6(2):4-9.

\section{Objectives}

1. Demonstrate how a collaborative group investigated and developed a safe way to expand diabetic nursing care to students in rural schools through the use of technology

2. Explain the challenges schools face in providing health services to their students

3. Discuss the public policy changes that resulted from this investigation of care delivery, and suggest additional ways to expand health services to overcome additional challenges

\section{Track: Operations Room 200 AB}

\section{SUNDAY, MAY 15, 2016}

\section{2:00 PM-1:00 PM}

Sunday, May 15, 2016

Session 7

\section{Session Title: TELEMEDICINE AND POPULATION HEALTH: CASE STUDIES IN BOLIVIA, BRAZIL, AND FRANCE}

MODERATOR: Xavier Urtubey, MD, MBA, Chief Executive Officer AccuHealth, Santiago - Las Condes, Chile
SHARE OF TECHNOLOGICAL COMPONENTS ON INNOVATIVE TELEMEDICINE PROJECTS IN THE LARGEST FRENCH REGION

PRESENTER: Christophe Couvreur, Ing, MBA, Program Director, Ile de France Region Telemedicine Program ${ }^{1}$, Yann L'Hermitte, MD, Medical Referent of Telemedicine Project ${ }^{2}$, Sebastien Leclerc, Senior Project Manager $^{2}$, Chloé Wozniak, Project Manager ${ }^{2}$, Chul Hong, Project Manager ${ }^{2}$, Julien Parigot, Project Manager², Clémence Charon, Project Manager², France Woimant, $\mathrm{MD}^{3}$, François Dolveck, MD, Head of Medical Departement ${ }^{2}$, Pierre Boiron, Head of GCSDSISIF ${ }^{2}$

${ }^{1}$ GCSDSISIF,IT Project Management Group funded by French Health Organization, Paris, France, ${ }^{2}$ GCSDSISIF, Paris, France, ${ }^{3}$ French Regional Health Agency of Paris, Paris, France

Introduction: The analysis of many Telemedicine activities deployments shows that the implemented technology is rarely shared. Thus, many hospitals find themselves with multiple technological solutions to practice telemedicine: a solution for tele-stroke, another for mental healthcare (autism, multiple disabilities ...) or for tele-pathology. In a difficult health economic environment, is the sharing of technological components on innovative telemedicine projects vain?

Methods: A regional group (GCS D-SISIF) consisting of health facilities, medical and social structures, prison facilities and primary care in the Ile de France region (12 million inhabitants) steers the implementation of a telemedicine technological platform, based on developments made by manufacturers. In order to achieve the technological sharing, governance is centered around three themes: the search for the economic sustainability of the platform concerning its operation, cost control related to expenditure arising from new developments to incorporate new projects, and finally securing the platform. For sustainability, a SaaS economic model was jointly built with manufacturers, model balanced between a bearable cost by the platform member structures to limit their initial investments that could be an obstacle to the development of their telemedicine project and profitability for the manufacturers. This model avoids the risks of models based on grants to fund the operation. For the control of development costs, a partnership innovation has been established with industry, win-win partnership where the functional needs of medical projects promoted by a medical carrier fuel specifications. The code remains the property of the industrial and in return the cost of development and maintenance of new developments thus produced are supported by the industry. For securing the platform, manufacturers have an obligation to respect national safety standards (hosting data of health that integrates iso13xxx) and national health professionals directory (which guarantees the recognition of inter regions degrees) and an obligation to respond to the audits led by the regional group.

Results/Discussion: The group now consists of nearly 100 members of the telemedicine platform called ORTIF. ORTIF is a full web platform whose offer is a catalog of services allowing medical data sharing, transfer and consultation of medical images, videoconsultation and use of collaborative functions between multiple players and several sites. The access to the platform economic model is a subscription cost based on selected services and the expected service level (Service Level Agreement) by the member structure. The subscription cost is capped, allowing structures a visibility on cost with an unlimited act volume. The innovation partnership is based on a principle of development bandwidth reserved annually by the manufacturers for the regional group. ORTIF first version has been implemented in October 2014 for uses in tele-stroke, and teleneurosurgery, teleradiopediatric. In 2015, new features have been added to meet new mental healthcare and teledermatology projects needs between 11 primary care for autistic, 17 primary care (in jail) and severely disabled children and 5 hospitals. An audit has been carried out and it has allowed to verify standards compliance, to identify areas for improvement in terms of ergonomics with a view to a national opening of the platform. 


\section{ORAL PRESENTATIONS ABSTRACTS}

Conclusion: Technology pooling seems possible provided that a governance of evolution of the technological platform is in place in which a balanced research of interests converge between manufacturers, regional group and the group members (hospitals, primary care) Prospects for applications related to the pooling of ORTIF platform in 2016 are telepathology, diabetes and retinopathy, and home monitoring for chronic care. The pooling opens saving outlook opportunities for technological establishment of new telemedicine projects, estimated between 200 and $400 \mathrm{~K} €$ per project and reduce the time of implementation between 2 and 4 months per project. The effects of pooling are not limited to technology, but also affect the change management tools in place (training and communication materials, e-learning). This will contribute to further improve the profitability of investments.

\section{Objectives}

1. Save outlook opportunities for technological establishment of new telemedicine projects

2. Reduce the time of implementation of telemedicine project

3. Capitalize on the change management tools in place

TELEMEDICINE IN BOLIVIA TO IMPROVE POPULATION HEALTH: A CASE STUDY DESCRIBING THE ACHIEVEMENTS AND CHALLENGES INVOLVED IN THE IMPLEMENTATION OF A NATIONWIDE PROGRAM

PRESENTER: Alfredo Levy, Master in Public Administration, Executive Director

ITTI Bolivia S.A., La Paz, Plurinational State of Bolivia

The main problem in rural Bolivia has always been limited access to specialized healthcare. This sparsely populated country extends over an area, roughly the size of Spain and France combined, characterized by rough terrain and poor civil infrastructure. In addition, health posts in rural areas are typically manned by young, inexperienced doctors or just a nurse. Thirty- four percent of the population lives in rural areas. Another large percentage lives in semi-rural areas, with also limited access to healthcare.

The telemedicine program in Bolivia can improve the life of over three million Bolivians.

In early 2013, the Ministry of Health of Bolivia, in an effort to improve the state of the healthcare system in the country, established, as a priority, the implementation of a nationwide, telemedicine program. Recent advances in telecommunication and information technology in the country, set the stage for providing an integrated solution, designed to maximize social impact.

Expected short and long term benefits of the telemedicine project are:

- improvement in coverage and quality of the care provided;

- democratization of specialized care;

- initiation of digitized medical history registry;

- establishment of education processes and procedures for healthcare givers;

- improved epidemiological monitoring and control;

- stream lining administrative processes.

In order to design and implement the project, the Ministry of Health teamed up with two cross- sector partners- International Telecommunication and Technology Integration S.A. (ITTI), a private Bolivian corporation, and Entel S.A., a public telecommunication company, owned by the State. Successful implementation and operation of the telemedicine network requires a high level of coordination between all parties involved, including the supplier of telemedicine equipment and software.

The Ministry of Health is responsible for the overall design of the project, supervision, assignment of medical personnel, and legislation initiatives. It designated three hundred and forty sites across the nine administrative departments of Bolivia to be equipped, trained and operationalized. These sites have a fourtiered classification. There are 269 remote TEL1 sites with five medical devices plus video conference equipment; 61 intermediate TEL 2 sites with 8 devices; 9 TEL3 sites located in the departmental hospitals with just video conference equipment; one TEL4 site in the ministry of health equipped with computers and servers.

ITTI's role consists of providing, installing and configuring, medical devices, as well as hardware and software equipment and programs. In addition, ITTI produces training materials, trains and certifies users. It provides a support desk that is accessible around the clock. It is also responsible for corrective and preventive maintenance of the equipment.

Entel's role is to provide connectivity through a secure network with a broadband superior to $1 \mathrm{Mbps}$ symmetric. It currently provides three connectivity solutions- fiber optics, microwave, and satellite.

Deciding which sites qualify for TEL1 or TEL2 equipment was a function of population density around the site and the quality of civil infrastructure. The decision to standardize equipment across all TEL1 and TEL2 sites was meant to facilitate training and management; and the type of medical devices was determined based on the most common health problems identified in Bolivia.

To date (9/11/2015) we can point out the following progress:

- The telemedicine network is operational in 6 of the 9 Departments;

- 120 sites (35.2\%) are fully operational;

- 251 sites (73.8\%) are fully equipped;

- 225 sites (66\%) with tested connectivity;

- 157 sites (46.1\%) with trained users;

- 2500+ telemedicine consultations.

These results indicate that one of the main constraints on the timely implementation of the project and its sustainability has to do with human resources. The Ministry is having difficulties recruiting and assigning doctors for training, particularly to remote locations. Furthermore, in some cases, because of the low IT proficiency of the doctors, ITTI's basic training is not sufficient to ensure an efficient operation. To overcome this challenge, the Ministry can introduce incentives to their recruitment program, develop a continuing education/training programs that go beyond what is currently provided by ITTI, and develop protocols.

Other constraints have to do with costs. In Bolivia an adequate satellite connectivity is cost-prohibitive at the present. Until costs come down, the mode of operation for $13.5 \%$ of the sites will be "store-and-forward." Furthermore, the Ministry, initially, assumed the cost of implementation and operation of the network. It is now faced with the challenge of gradually transferring financial responsibility to the municipalities.

The Ministry is working hard to educate the public on the availability of Telemedicine in an environment where traditional medicine, curanderismo is the preferred choice. But more effort needs to be made to launch public awareness campaigns about the benefits that telemedicine will bring them.

The network is expected to be fully operational by early 2016. Future developments may include increasing the density of sites and creating links to specialized hospitals, abroad, for special consultation cases.

\section{REFERENCES}

1. Ministerio de Salud del Estado Plurinacional de Bolivia, Huanuco R., Maturano M.; 2013; "Proyecto Telesalud Para Bolivia"; La Paz - Bolivia.

2. Organización Panamericana de la Salud (OPS), Doctor Jack Antelo Solis; Evolución y situación actual de los distritos de salud en Bolivia.

3. Programa de las Naciones Unidas para el Desarrollo (PNUD), Verónica Paz Arauco; Los cambios detrás del cambio Informe Nacional sobre Desarrollo Humano; 2010, La Paz Bolivia.

\section{Objectives}

1. Identify similarities and differences between the Bolivian case and attendees own environment, with respect to the conditions for implementing a Nationwide telemedicine program

2. Adapt some of the lessons learned from the Bolivian case and be able to anticipate political, cultural and technical challenges, in their own environment 


\section{ORAL PRESENTATIONS ABSTRACTS}

3. Apply technical factors involved in implementation, and select the right technology, platform and partnerships as learned from the Bolivian case

\section{CAN TELEMEDICINE INCREASE WORKFORCE DURING AN EPIDEMIC?}

PRESENTER: Milton Steinman, MD, Telemedicine Coordinator, Leonardo Lima Rocha, MD, Renata Albaladejo, RN, Gustavo Amorim Cunha, Alexandre Marra, MD, Alberto Kanamura, MD,

Oscar Pavao dos Santos, MD, Oscar Fernando Pavao dos Santos, PHD

Hospital Israelita Albert Einstein, Sao Paulo, Brazil

Introduction: Dengue is a viral infectious disease caused by an arbovirus from Flaviviridae family. In the first semester of 2015, Brazil had the highest incidence of dengue fever worldwide (179.24 cases/100,000 population), with 360,331 laboratory confirmed cases and 600 deaths. The number of notified cases of dengue fever in Sao Paulo was overwhelming, resulting in massive demand for medical assistance from a public system that already operated at its maximum capacity resulted in long waiting lines, restricted access to healthcare services and a shortage of hospital beds, both in public and private sectors. In this context, some emergency measures, such as the implementation of hydration tents, proved to be a key factor in epidemic control in order to lower the disease morbidity, the number of hospitalizations and if possible, the mortality rates. Purpose: To assess the use of telemedicine consultations performed by a physician to help reduce the burden for healthcare demand during dengue epidemics in Sao Paulo State in 2015.

Methods: A hydration tent were deployed in a high prevalence site in São Paulo to receive patients referred by primary care units and emergency hospitals with symptoms suggestive of dengue fever. The multidisciplinary team consisted for professionals from various sectors of our hospital (physicians and nurses) and was divided in 4 groups. If any major complication was detected, the patient was transferred to a tertiary hospital. Over the study period, there were no patient deaths among those treated at the hydration tent. The 5th group (TM group) was composed by a telemedicine cart (encrypted IP communication and audio \& video using videoconference) staffed by a senior nurse supported by emergency medicine physicians located 20 miles away, at Hospital Israelita Albert Einstein. Every decision making was based upon standard protocols. Effectiveness of telemedicine was studied by means of the proportion of service failures, considering failure as the need for the patient to receive personal assistance after consultation by telemedicine.

Results: A sum of 4,533 cases was seen during the taskforce. Thirteen telemedicine consultants performed 265 consultations in the TM group. Most of the patients were females, 146 (55\%), ranging from 20 to 49 years, 190 (71\%) from white, 104 (39\%), and brown, 96 (36\%) races. Patients with non-complicated dengue fever (Group A) were the most frequent risk classification found, 239 (90\%). First infection was present in 251 (94\%) of the cases. Additionally, a positive rapid dengue test (NS1 kit) was present in 184 (69\%). In 14 patients (5\%), was not possible to finish the TM consultation. The main cause of failure was due to some level of insecurity on finishing TM consultation, mainly related to comorbidity (5 patients), clinical findings (4 patients) and transitory response to hydration (5 patients).

Conclusions: A major contribution of this approach was to demonstrate that simple, low-cost telemedicine program provided safe, high-quality and costeffective primary care services under the direction of a doctor in the care of patients with dengue fever during the epidemics in São Paulo, increasing workforce. TM assistant concept might provide epidemics anywhere with a novel strategy for addressing its medical workforce shortage, particularly in rural and remote settings.

\section{REFERENCES}

1. Marra $A R$, de Matos $G F$, et al. Managing patients with dengue fever during an epidemic: the importance of a hydration tent and of a multidisciplinary approach. BMC Res Notes 2011; 4:335.

2. Pan American Health Organization. Number of Reported Cases of Dengue 2015. http://www.paho.org/hq/index.php?option=com_topics\&view=article \&id= $1 \&$ Itemid $=40734$

\section{Objectives}

1. Study telemedicine during epidemics

2. Discuss strategies in dengue fever

3. Verify efficacy of telemedicine

\section{SUNDAY, MAY 15, 2016}

1:15 PM-2:15 PM

Sunday, May 15, 2016

\section{Session 15}

\section{Session Title: INTERNATIONAL TELEMEDICINE}

MODERATOR: Humberto J. Alves, MD, PhD, Vice Dean School of Medicine Federal University of Minas Gerais, Belo Horizonte, Brazil

\section{IMPLEMENTING BEST TELEHEALTH PRACTICES INTERNATIONALLY}

PRESENTER: Tomas J. Sanabria, MD, Cardiologist

Centro Medico De Caracas, Caracas, Venezuela, Bolivarian Republic

“Implementing Best Telehealth Practices Internationally" Canada, the USA and Latin America are moving from theoretical discussion of implementing telehealth to initiatives that improve outcomes and lower costs throughout the continuum of care. The joint effort by medical leaders, international institutions and private companies is demonstrating how knowledge of the practical uses of telehealth in one country can benefit others and improve the collective experience. The ATA Special interest Groups for International and for Latin America have long been at the forefront of similar efforts to share knowledge of use to all. The elements of this international collaboration are: - Best practice Clinical protocols, specially designed for remote application and for adaptation, translation and "tropicalization" to localities and changing health needs. - Common Operating Systems operating off of the cloud and able to connect clinicians in remote sites with Internet connections anywhere on the globe. - Knowledgeable and experienced clinical, management and technical teams in the uses of remote health monitoring and care. The intent of this presentation is to discuss practical measures now underway to improve access to better health for all. Participants in his freewheeling discussion will include experts from Venezuela, Peru, Canada, Haiti and the United States who have been collaborating on implementation of telehealth projects now benefitting over 10 million people. Applications: A Three (3) level model of Telemedicine (Maniapure model) initiated two decades ago has become a "leapfrogging" experience now expanding in different Latin American countries over which primary care and educational experiences have been added and is showing unlimited possibilities of up-scaling to reduce costs in healthcare, adding previously excluded communities (ecosystems) to adequate medical attention and services and giving subspecialty access to almost anyone in the system. The concept of VIRTUAL TRIAGE CENTER (level 2) differentiates from a routine "call center" being the operative brain of the system where anyone requesting information in health related science/services (level 1) can converge, and through which the more sophisticated health related subspecialty information/opinion (level 3 ) is matched with the "consulting" part. The 3 components for the concept: (a) Technology, (b) Processes (operating model changes) and the needed, and (c) Behavioral changes, which are dynamically evaluated and are adapted for each situation. Following this adaptability (our goal has been cost/effectiveness) we 


\section{ORAL PRESENTATIONS ABSTRACTS}

have been able to work with almost any technological equipment (brands). We provide services to any level of healthcare professional, patients (as end users), and nursing homes, chronic hospitals, insurance companies and many others Our model allows store-and-forward or real-time service, we give top priority to imaging and are very conscious about connectivity and bandwidth restrictions so have to dynamically migrate with changing sensor technology. A functionally adaptation and integration of software to provide diagnostic/therapeutic assessment to health professionals versus providing disposition advice to patients/insurance institutions all these add on to a joint partnership an over 35 years of experience being adapted to many regional and international regulations.

\section{Objectives}

1. To prove that cooperation with different experiences and model can accelerate the resolution of chronic difficulties

2. Each group of barriers has a different solution, even if using the same scheme, some problems and solutions can be common but many are different and have to be managed as such

3. Including our different operations on a matrix resulting from detail analysis can accelerate the management of cost and exclusion problems

IS THERE A NEED TO PLAN TELEMEDICINE PROGRAMS? A NEW MODEL FROM THE DEVELOPING WORLD: PART 1

PRESENTER: Maurice Mars, MBChB, MD, Professor of Telehealth Nelson R. Mandela School of Medicine, University of KwaZulu-Natal, Durban, South Africa

Many telemedicine projects fail to become sustainable programs and much has been written about factors that need to be addressed to achieve success and sustainability. These include: needs assessment; e-readiness assessment; development of business, implementation, change management, monitoring and evaluation, and scale-up plans; and ongoing program refinement. Uptake of telemedicine has been particularly low in the developing world as telemedicine adds extra steps to the workflow of already overburdened health professionals with limited infrastructure and government support. We have noted the spontaneous development of telemedicine services in several disciplines in the public sector in South Africa where doctors in government hospitals are salaried. This paper reports the evolution of two such spontaneous, unplanned, mobile phone based services and identifies aspects of these services which require improvement.

Case Study 1: m-Dermatology

The dermatology department at the Nelson R Mandela School of Medicine has run a synchronous teledermatology program since 2005. Previous attempts to establish e-mail store-and-forward telemedicine for doctors at rural hospitals (2001) and residents in training (2008) failed. In 2013, the dermatologists reported that their residents had begun sending them text messages and photographs via WhatsApp with requests for advice on diagnosis and management of problem cases. They responded via WhatsApp and saw this as an additional and beneficial form of training. This was not planned and occurred because the junior doctors had unmet needs and found a simple solution - taking photographs with their phones and sending them together with text messages. This informal service has spread and doctors at several rural hospitals now regularly send cases. The senior dermatologists are happy to respond as they are reducing the number of patients referred to their clinics. While they estimate receiving 1-2 cases each per day and claim reduction of referrals by about 70\% they have no data to support this. The service is unstructured and unregulated. Some dermatologists keep images and text messages as WhatsApp chats, some delete all images and texts while others only keep cases of interest. What happens to the images and text messages from referring doctors in unknown. The referring doctors and the dermatologists are satisfied with the service being provided and see it growing.

\section{Case Study 2: m-Burns}

The burns unit at Inkosi Albert Luthuli Central Hospital in Durban has 45 beds and serves approximately 5 million people. For admission to a unit's bed or the burns clinic the referring doctor must telephone the burn surgeon on call to discuss the case following a pre-admission pro-forma, after which a decision is made on acceptance of the patient or further management at the referring hospital or clinic. For the last three years, when burns surgeons have been unable to attend wound dressing changes because of staff shortages and surgical commitments, junior doctors have photographed the wounds on their mobile phones and taken them to the operating theatre to show the surgeons and receive management advice. Based on this experience the burns surgeons have since December 2014, required referring doctors to submit photographs of the burn wound taken using mobile phones and send them by MMS or WhatsApp, after completion of the telephone conversation and pro-forma. The decision on further management is then sent to the referring doctor as an SMS or WhatsApp message. For patients who require further resuscitation before transfer the burns surgeon stays in regular telephonic contact. The information gathered during the phone consultation and the photographs are entered and saved in password protected database which is backed up on a secure hospital server. The images are then deleted from the surgeons' phones. In the first eight months of this service 119 cases were referred. Photographs changed the management of $70 \%$ of cases with admission avoided (47\%) or postponed (23\%). Prior to this service approximately 30\% of the referrals accepted to the burns unit were inappropriate because of overestimation of the burn area and depth by the referring doctor as were $60 \%$ of the referrals to the clinic.

Conclusions: These services were unplanned. They show that telemedicine services can develop without following the many steps advocated. They began with trainees within departments using their phones for photography and messaging to solve their problems, with all costs absorbed by the doctors. Different approaches have been taken to record keeping, reporting, data security and confidentiality. Whether consent for the teleconsultation is obtained is unknown and authentication remains a problem. Careful assessment of the ethical and other shortcomings of each service is required and procedures and processes need to be implemented to address these. We are aware of spontaneous telemedicine activity in ophthalmology, orthopaedics, intensive care, radiology, cardiology (ECG) and psychiatry. This simple approach may facilitate provision of telemedicine in the developing world but is unlikely to work in the more regulated environs of the developed world.

\section{Objectives}

1. Identify new ways of providing telemedicine services without the need for prior planning

2. Describe telemedicine services that have evolved spontaneously

3. Summarize the strengths and weaknesses of spontaneous telemedicine services

IS THERE A NEED TO PLAN TELEMEDICINE PROGRAMS? A NEW MODEL FROM THE DEVELOPING WORLD: PART 2

PRESENTER: Richard E. Scott, BSc 1st (Hons), PhD, Professor Nelson R. Mandela School of Medicine, University of KwaZulu-Natal, Durban, South Africa

Subject Matter Solutions suitable for the developing world must be designed and developed in alignment with and recognition of the prevailing setting, however the realities of the developing world are often understated or misrepresented. The developing world struggles with dire health circumstances and consequences. Issues extend across the globe (Places), and impact several clear issues (Problems), many of which might be facilitated through technologically appropriate use of telehealth (Possibilities). This presentation will provide perspective on Places, Problems, and Possibilities from around the world, highlighting the reality of developing world issues and needs. The health dilemma of the developing world is most evident in sub-Saharan Africa, 
with $24 \%$ of the global burden of diseases serviced by just 3\% of the world's healthcare workers with access to merely $1 \%$ of world health expenditure. Problems: The WHO identified 5 developing countries in Latin American and the Caribbean, 12 in Asia, and 39 in Africa facing severe shortages of health workers. Many developing world health systems are hampered by a desperate lack of human health resources in absolute number, skills, and specialty. In many parts of the world, rural health centers are run by unqualified health workers. Community Health Workers have been suggested as a solution through task shifting and more training, but many are unpaid, have minimal education and literacy, and are already pressed with current activities. The world is becoming urbanized with half of humanity living in cities, and about one quarter of them ( 1 billion) in poor health in slums. There is exaggeration of the penetration of mobile phones (often said to be 'ubiquitous'), erroneously implying that vulnerable and poverty stricken groups (those most in need) have significant ownership, ready access, and ability. Possibilities: Technologically appropriate and culturally sensitive telehealth solutions implemented at household, community, societal, national, and international levels could address some of these problems, whilst other issues must be tackled at similar levels but through changes in policy and practice. How might telehealth (ehealth) help the developing world? It must focus on health, health system, and health service NEEDS, some of which have been highlighted above. These and others will be presented and discussed in the workshop. The goal is to raise awareness and dedication of participants to identifying and implementing needs based and innovative telehealth solutions that successfully and sustainably respond to the realities of the developing world.

Objectives

1. Gain an understanding of the realities of the developing world to be considered in telehealth applications

2. Gain an Appreciation that the least expensive and simplest technologically appropriate solutions are required in the developing world

3. Gain insight into the needs of the poverty stricken and vulnerable groups and how health 'systems' and contexts differ significantly between developed and developing world countries, as will their telehealth solutions.

\section{MONDAY, MAY 16, 2016}

8:00 AM-8:45 AM

Monday, May 16, 2016

\section{Session 23}

Session Title: DEMONSTRATING VALUE BASED

ON A DATA-DRIVEN APPROACH TO BUILD THE

\section{RETURN ON INVESTMENT MODEL FOR TELEHEALTH}

PRESENTER: Najib Ben Brahim, PhD, Chief Executive Officer and Founder Telehealth Management, Charlottesville, VA

PRESENTER: Karen Rheuban, MD, Professor and Senior Associate Dean for CME, Director

Pediatrics Continuing Medical Education and External Affairs, Center

for Telehealth at University of Virginia, Charlottesville, VA

Background: The exponential growth of telehealth programs nationwide reflects a host of factors that include technology innovation, greater broadband deployment, provider acceptance, consumer demand and an increasingly favorable public policy environment. It has been reported in 2012 that nearly half of U.S. hospitals engage in some form of telehealth (1). The benefits of telehealth include expanding access to high quality of care, improved triage, a reduction in healthcare costs, and mitigating geographic and socioeconomic barriers to care. Problem/Opportunity: As healthcare systems, providers and payers seek to analyze a host of factors related to investments in and outcomes related to telehealth, the need for a standardized framework for performance reporting/ assessment and quality assurance for telehealth services and programs has become apparent. (2)

Methods/Solution: Based on data continuously captured over the last 20 years of operations, a state of the art, data-driven, HIPAA compliant, integrated approach to the development and implementation of an ROI model for telehealth was implemented at the University of Virginia Health System. This model, designed as an automated data collection tool captures data from the electronic medical record, institutional financial systems, clinical videoconferencing call logs, and patient registration databases, to combine the clinical, financial and operational data for the telehealth program. In addition, processes and algorithms were defined to measure and report the performance of different telehealth service lines. The ROI tool can track both start-up and operational costs, and measure direct \& indirect revenue streams (fee-for-service, contracts, savings and downstream revenue) to the program. As a pilot project, we also linked the data analysis tool with clinical quality metrics for our telestroke program.

Conclusion: Based on a data-driven approach, the University of Virginia Center for Telehealth is now able to accurately measure and report performance metrics of the telehealth program. This methodology provides standardized data collection processes, and analytic tools to guide and support institutional investments in telehealth and requisite quality reporting requirements.

Key words: Telehealth, Telemedicine, ROI, standards

\section{REFERENCES}

1. Institute of Medicine. The role of telehealth in an evolving healthcare environment. Washington, DC: National Academies Press, 2012.

2. McLean $S$, Sheikh $A$, Cresswell $K$, et al. The impact of telehealthcare on the quality and safety of care: a systematic overview. PLoS One 2013; 8(8):e71238. Objectives

1. Demonstrate the value of telehealth based on a data-driven approach currently implemented at the University of Virginia Health System. The data used for the analysis spans more than 20 years of operations at the UVA Center for Telehealth.

2. Define and measure the return on investment (ROI) of telehealth and link it to quality of care metrics

3. Define the ROI model for telehealth and identify standards for quality assurance and business key performance indicators for telehealth. Discuss the implications of deployment of such a model in large institutions

MONDAY, MAY 16, 2016

11:00 AM-12:00 PM

Monday, May 16, 2016

\section{Session 31 \\ Session Title: INTEGRATING TELEMEDICINE INTO THE HEALTHCARE DELIVERY SYSTEM}

MODERATOR: Edwin Vaughan, MBA, President

CHI Virtual Health, Englewood, CO

PRESENTER: Randall Moore, MD, MBA, President

Mercy Virtual, Chesterfield, MO

TEN STEPS TO OPERATIONALIZING TELEHEALTH WITHIN

A HEALTH SYSTEM

PRESENTER: Anya Sanchez, MD, MBA, Enterprise Director, Special Projects

UC Health, Cincinnati, $\mathrm{OH}$ 
The application of telehealth (or "connected care") technology has been accelerating rapidly over the past decade. However, many healthcare organizations struggle to integrate connected care technologies into existing operating systems and care delivery models. This may result in telehealth programs that are isolated from mainstream operations and disconnected from the care delivery model. Within our organization, we came to refer to these as "pop up" programs, because they existed within their own separate ecosystem.

As we considered the huge spectrum of telehealth applications along the patient care continuum, encompassing different end-users, sites of care, and patient populations, we quickly realized that a "pop-up" model would not be sustainable. The scale of building a series of isolated, stand-alone programs would not be economically feasible, and would introduce other problems that arise from a complex and highly fragmented system. Instead, we needed to build an integrated, simple system that would mesh with our existing care delivery model in a way that was economical and low risk. In addition, we needed to ensure that telehealth served our strategic priorities (instead of becoming a strategic priority in itself) and fit within the operational context of our various care environments. We needed to determine what infrastructure or platform all telehealth programs required in order to be successful, and then provide that support in a way that was scalable and economical.

"Ten Steps to Operationalizing Telehealth" outlines 10 practical and actionable steps to building an enterprise-level approach to the integration of telehealth into a health system's care delivery model. We identified these steps by carefully assessing the evolution of our telehealth program at UC Health, and identifying critical junctures which we needed to navigate in order to move telehealth forward within our institution. Despite the fact that these are subjective learnings from our team, we have received enormous interest from other institutions in the methodology by which we've rapidly advanced our telehealth capabilities in a relatively short period of time. Our progress has been noted by third-party telehealth experts, such as Chicago-based healthcare think tank, Sg2. Several of the steps outlined in this presentation were highlighted in Sg2's recent publication, Virtual Health, Taking the Next Step.

To date, our approach has demonstrated significant success, as measured by the number of telehealth programs currently in design, implementation, and active pilot phases. Another objective measure of success includes the variety in the types of programs we have in development across the care continuum. This provides support to the flexibility of our telehealth infrastructure in being applicable and effective across various end-users and sites of care.

\section{Objectives}

1. Demonstrate awareness of the changing healthcare environment and the need to integrate telehealth capabilities into health systems

2. Describe 10 actionable steps to build a telehealth infrastructure within a health system that is both strategically aligned and cost effective

3. Assess the short-term and long-term effectiveness of a telehealth infrastructure

MONDAY, MAY 16, 2016

1:15 PM-2:15 PM

Monday, May 16, 2016

\section{Session 37 Session Title: INTEGRATING TELEHEALTH CLINICAL MODELS WITHIN THE EMR AND ACROSS THE CARE CONTINUUM}

PRESENTER: Steven Dean, MS, Director of Telemedicine Inova Health System, Falls Church, VA

PRESENTER: Edward Loo, MSECE, Telemedicine Engineer Inova Health System, Falls Church, VA

After a decade of telehealthcare delivery, many new clinical models have emerged to support service lines within an urban health system. This effort required a wide scope of clinical expertise and coordination to create a system of programs linking providers and patients via electronic communications. Clinical areas such as emergency departments, critical care, neurosurgery, pediatric ICU and behavioral health are supported with remote clinical teams using synchronous technology. The purpose of this presentation is to describe one organization's journey in developing robust telehealth services in many specialty areas and its subsequent integration of those capabilities inside an EMR.

The team will describe essential processes and lessons learned such as program coordination, stakeholder engagement, and change management techniques impacting successful EMR integration and technology acceptance. The value of communication to promote consistency and understanding cannot be underestimated to sustain clinical programs.

Learn how various models leverage the same infrastructure in different capacities to deliver clinical practice in settings across the care continuum. Multiple programs will be described and how technology was matched to meet the clinical needs of the patient.

Leadership role characteristics essential to telehealth program development and sustainment will be identified and discussed. Leaders in both the telehealth and clinical arenas play influential roles in change management, ongoing development, implementation and sustainment of programs. Communication and technical barriers will be explored and solutions for enhanced communication will be described.

Length of stay, time to treatment, and encounter data will be shared to illustrate utilization and outcome metrics. This presentation will also highlight innovative ideas in consideration for future telemedicine solutions.

\section{Objectives}

1. Demonstrate the migration and integration of telemedicine services inside an EMR

2. Deliver outcomes and metrics highlighting program success

3. Identify barriers and outline effective leadership roles to ensure program success

\section{Room 211 AB}

\section{MONDAY, MAY 16, 2016}

1:15 PM-2:15 PM

Monday, May 16, 2016

\section{Session 84 Session Title: ROUNDTABLE SESSION}

\section{Roundtable 2}

\section{DEVELOPING A MULTI-NATIONAL TELEMEDICINE COLLABORATION}

\section{STANDARD}

PRESENTER: Peter Killcommons, MD, CEO

Medweb, San Francisco, CA

In an effort to improve collaborative medical response to natural disasters or population displacement, a project was developed to evolve a collaborative mechanism for telemedicine consultations. This panel draws from initial experiences in this process and invites feedback and the development of additional efforts in this area.

\section{Objectives}

1. Present initial findings from a multi-national telemedicine effort

2. Discuss standards used and develop a dialogue around further evolution of technical standards

3. Invite additional institutions to participate in an expanded study in this area of collaboration 


\section{ORAL PRESENTATIONS ABSTRACTS}

\section{Roundtable 3}

\section{IMPROVING TELEHEALTH BUSINESS PERFORMANCE USING PROCESS MANAGEMENT TOOLS AND HUMAN FACTORS}

PRESENTER: David Ben-Arieh, PhD, Professor, Industrial Engineering Kansas State University, Manhattan, KS

This roundtable will briefly discuss the connection between operational parameters of any telehealth system such as arrival rate, service rate and variability on the actual performance of such a system. The audience need not have a background in operation research or production management as these topics will be covered just enough to provide a substantive link between the parameters and system's performance. In this roundtable I will also introduce the concept of Human Factors as a necessary ingredient for successful operation of any telehealth practice.

I will also link the financial performance or the business aspect of a practice to these issues.

Issues such as direct and indirect costs of providers, resources and patients waiting times, office preparation times and providers' productive and nonproductive times will be discussed.

Expected results include understanding the operational parameters of a telehealth system and the effect of these parameters on the system's performance including utilization and throughput.

Also the participants will be able to connect the Human Factors issues to operational parameters and then to the financial performance of the telehealth operation including revenue and cost. This will allow the practitioners to design and tune-up the process for the best financial outcomes.

\section{REFERENCES}

1. Wickens $C D$, Lee JD, Liu Y, Becker, SEG. An Introduction to Human Factors Engineering, 2nd Edition, Pearson Prentice Hall, 2004

2. Hopp JW, Spearman ML. Factory Physics, 3rd Edition, Waveland Press Inc., Long Grove IL, 2008

3. Ben-Arieh D, C-H Wu, 2015 Optimal Surgery schedule based on PICU Nurses workload, Healthcare Systems Process Improvement (formerly SHS conference), February 18-20, Orlando FL.

4. Ben-Arieh D, Ryan Aeschliman, 2015, Implementing Telemedicine Processes modeling and analysis, Healthcare Systems Process Improvement (formerly SHS), February 18-20, Orlando FL.

\section{Objectives}

1. Present process improvement concepts such as Lean operations and six sigma and their impact on telehealth operations

2. Discuss the relations between process efficiency and effectiveness and the telehealth financial performance

3. Discuss the importance of Human Factors considerations on the success of telehealth practices

\section{Roundtable 4}

SHOW-ME ECHO: IMPLEMENTATION OF A STATEWIDE CAPACITY BUILDING PROGRAM

PRESENTER: Rachel Mutrux, BA, Director, Telehealth Network University of Missouri, Columbia, MO

Introduction: America's Health Rankings show that in (2014), Missouri ranked 36 of the 50 states $^{1}$. Counties located in the southern portion of the state rank among the worst in the state for all health outcome rankings ${ }^{1}$. According to a Missouri Foundation for Health Report from 2009, 40\% of Missouri's population live in rural areas, but only 25\% of Missouri physicians practice in rural areas ${ }^{2}$. Traditional telemedicine works to reduce access issues in rural and underserved Missouri, but is limited to a 1:1 ratio of providers to patients seen at any given time. Show-Me ECHO (Extension for Community Healthcare Outcomes) changes the ratio by not only increasing access to specialty care, but increasing the capacity of specialty care available. It works as an effective triage system for specialty care by shifting only the highest acuity patients to the specialists. It provides much needed medical educational credits to participating primary care providers free of charge while forging professional relationships. ECHO disseminates best-practices and evidencebased treatment recommendations. Additionally, ECHO will provide an economic development benefit to participating communities by allowing healthcare dollars to be spent locally.

Background: Dr. Sanjeev Arora, hepatologist at the University of New Mexico, developed a transformational program that increases capacity for specialty care in rural and underserved areas called Project ECHO. Started in 2003, ECHO is a type of virtual grand rounds that brings together a voluntary group of primary care providers (PCPs) with an interdisciplinary team around a specific disease state or condition. The participants meet with the specialist team at a regularly scheduled day and time using telehealth equipment. Deidentified cases are presented and discussed, recommendations are made, and outcomes are measured. The original Project ECHO was designed to increase the number of providers treating patients for Hepatitis C. With the great success of his project, Dr. Arora envisioned the model being used by many different conditions and diseases where PCPs partnered with interdisciplinary teams from academic medical centers. The model has been published on, has won awards, and is spreading around the globe ${ }^{3}$. The Missouri Telehealth Network (MTN) began in 1994 to improve rural and underserved access to specialty medical care ${ }^{4}$. Our model, which has evolved over the years, is to partner with many different types of healthcare organizations around the state to assist in starting telehealth programs and to offer services from the University of Missouri when appropriate. Because of our operational model, the ECHO project has layered perfectly onto existing services and utilizes existing staff in similar roles. In January of 2014, with the help of current and retired legislative champions, MTN signed a replication agreement with the University of New Mexico with the vision of creating a state-wide ECHO umbrella organization.

Methods: The breaking point in the success of Show-Me ECHO was when our legislative champion introduced legislation that would create Show-Me ECHO under MTN. We worked with the Missouri legislature to add a $\$ 1.5 \mathrm{M}$ appropriation for Show-Me ECHO. We were not able to obtain state funding during the first year, however, stakeholders helped to fund two ECHO pilot projects, Chronic Pain Management and Autism. The pilots ran from fall 2014 to fall 2015. Pilot topics were chosen largely due to the buy-in from the expert teams. Both of the pilots were successful in recruitment of willing PCPs, and in getting the operational team thoroughly tested. During the 2015 legislative session, the Show-Me ECHO state appropriation of \$1.5M back was reintroduced and signed into law in June, 2015. We have started/continued 6 ECHO projects with the appropriated funds including Chronic Pain Management, Autism, Hepatitis C, Endocrinology, Childhood Asthma, and Dermatology.

Conclusion: The Show-Me ECHO projects will increase capacity to care for patients with specific disease states or conditions by creating mentoring relationships between PCPs and interdisciplinary expert teams. Outcomes of the project will be measured in terms of clinical outcomes for the deidentified cases presented during the ECHO 'clinics', by measuring selfefficacy of the PCPs, and by researching the MO HealthNet (Missouri Medicaid) claims data over time. This session will inform participants of the step-by-step implementation process in Missouri, lessons learned, challenges and success factors. 


\section{ORAL PRESENTATIONS ABSTRACTS}

\section{REFERENCES}

1. Missouri Annual State Health Rankings. America's Health Rankings. Web. Accessed 10 Sept. 2015.

2. Recent Studies and Reports on Physician Shortages in the US. 1 Oct. 2012. Web. Accessed 10 Sept. 2015.

3. Arora S, Thornton K, Murata G, Deming P, Kalishman S, Dion D, et al. Outcomes of treatment for hepatitis $C$ virus infection by primary care providers. $N$ Engl J Med 2011; 364(23), 2199-207.

4. Missouri Telehealth Network: General information. http:// medicine.missouri.edu/telehealth/general.html. Accessed 10 Sept. 2015.

\section{Objectives}

1. Participants will demonstrate understanding of the phases needed for successful ECHO project implementation

2. Participants will use lessons learned to assess feasibility of implementing ECHO in their own area

3. Participants will be able to prepare a project plan for an ECHO program in a specific disease state

\section{Roundtable 5}

LOWERING THE COST OF HEALTHCARE DELIVERY VIA VIRTUAL CARE

PRESENTER: Ronald F. Dixon, MD, MA, Medical Director, Director of Virtual Practice Project, Assistant Professor of Medicine, Harvard Medical School, Director of Center for the Integration of Medicine and Innovative Technology - Delivery System Innovation Program

Massachusetts General Hospital, Boston, MA

One of the challenges facing Partners Healthcare and other large provider organizations is the rising cost of healthcare delivery in the setting of decreased payments. This challenge is especially relevant to the chronic disease population which makes up the majority of expense of the delivery system and has been a target of both insurers and providers in the field of population management. At Partners, the population health management group has been asked to decrease total medical expenditures over the next 10 years. One of the ways of doing this has been identified as decreasing the total number of visits per patient to doctor's offices, in order to decrease overhead and overall utilization. Virtual healthcare delivery has been identified as having significant potential in decreasing the volume of visits to outpatient settings. We will examine and discuss a number of tested modes of delivering care virtually, covering key aspects for any organization considering these modes of care: regulatory/ reimbursement; impact on clinician resource utilization; organizational/financial barriers; ease of implementation. Asynchronous modes of care have demonstrated advantages at Partners in many of these criteria, and will be explored more closely.

\section{REFERENCES}

1. Dixon RF, Rao L. Asynchronous Virtual Visits for the Follow-up of Chronic Conditions. Telemed J E Health 2014;l 20(7):669-72.

2. Ganguli I, Wasfy JH, Ferris T. What Is the Right Number of Clinic Appointments? Visit Frequency and the Accountable Care Organization. JAMA 2015; 313(19):1905-906.

3. Dixon $\mathrm{RF}$, Stahl J. A randomized trial of virtual visits in a general medicine practice. J Telemed Telecare 2009; 15(3):115-17.

\section{Objectives}

1. Identify ways to decrease costs through virtual care delivery

2. Compare asynchronous and synchronous modes of virtual care delivery and their impact on your organizational goals
3. Diagnose potential barriers to adoption of virtual care in your organization

\section{Roundtable 6}

\section{RESOURCES AND OPPORTUNITIES IN THE GLOBAL HEALTHCARE SECTOR}

PRESENTER: Michelle L. Ouellette, MBA, Senior International Trade Specialist

U.S. Department of Commerce, US Commercial Service, Boston, MA

Mobile health and telehealth are appealing Health IT subsectors to examine for export opportunities. As mobile health applications continue to be a priority for research and development, technologies and affordability continues to improve at a rapid rate. Additionally, many countries are upgrading mobile network services, particularly those supported by large telecommunications companies.

Explore the global opportunities for telemedicine. Whether it is your first forray into exporting, or you would like to expand your business to additional markets, learn about opportunities via case studies coordinated via our global network of the U.S. Embassies and U.S. Consulates.

Discover the federal resources that the U.S Department of Commerce's U.S. Commercial Service offers to grow your international sales of U.S. products and/or services.

During the session, you will hear case studies outlining global opportunities for telemedicine, in addition to understanding more about the work that our posts in Africa are working on in coordinating with aid agencies working in rural Africa.

\section{REFERENCES}

1. http://trade.gov/topmarkets/pdf/Health_IT_Top_Markets_Report.pdf - July 2015 U.S. Department of Commerce, International Trade Administration - 2015 Health IT Top Markets Report.

\section{Objectives}

1. Demonstrate international telemedicine opportunities from metropolitan centers of excellence supporting rural areas

2. Analyze three case studies on three global telemedicine opportunities

3. Outline federal programs and resources available to assist U.S. telehealth companies

\section{Roundtable 7}

\section{LEVERAGING TECHNOLOGY TO ENHANCE PATIENT CARE IN AN INTEGRATED SPECIALTY SERVICES MODEL}

PRESENTER: James Reynolds, MBA, MHA, Director of Clinic Operations Sanger Heart \& Vascular Institute, Charlotte, NC

With rapid change in the healthcare environment and the similar pace of change in technological advancement we set out to marry to the two to transform the way we deliver care to our cardiology patients. The goals outlined for this project were to decrease cost of care to the patient, improve appropriate care scores, improve patient satisfaction, improve access to Cardiovascular (CV) services, and deploy a population health model for cardiovascular care utilizing virtual technology. We felt as though we could do this by deploying embedded personnel (RN, ACP, CMA) within primary care practices or Regional Care Centers (RCC) to facilitate cardiology teleconsults between patients and physicians at a centralized location or Integrated Practice Unit (IPU). The equipment used to perform the 
teleconsult includes a laptop, screen, and camera mounted on a mobile cart with wireless capabilities for flexibility coupled with an integrated stethoscope system allowing the MD to hear heart and lung sounds remotely. We also felt that with the amount of potential downtime between patient encounters there would be opportunity for those persons to have a positive effect on population health metrics of the host practice's patient panel in the realm of cardiology, CAD/IVD and CHF metrics specifically. To positively impact patient access we sought to reduce the median lag days to new patient visit using the Sanger Heart \& Vascular Institute (SHVI) Main campus as an internal benchmark. Patient satisfaction was measured through a short survey administered to patients post virtual interaction with emphasis on likelihood to utilize telemedicine in the future for care. Cost of care to the patient was measured as a decrease in miles/time travelled as compared to travelling to the nearest traditional care site. For the purposes of this presentation we will use data sets from 3 pilot practices representing the most complete and longest running information possible. These sites will be named for the host practice in each location Bessemer City Family Medicine, Eastridge Family Medicine, and Riverbend Family Practice (Mt. Holly). Across these three locations at the conclusion of the pilot phase, ending in July of 2015, there were 299 total virtual encounters across all three these locations. The majority, 156, being at the Riverbend Family Practice location with 98 and 45 visits at Eastridge Family Medicine and Bessemer City Family Medicine respectively. The virtual program was able to reduce the median time to new patient consult from 10 days at our benchmark practice to 3 days at the pilot sites or a reduction of 70\%. Across all three practices we had 100\% participants' say that they were not only satisfied with the level of care they received with our program but they would seek out this modality for future care as well. Most patients commented ease of access and the ability to connect close to home were the reasons for this decision. Across the three sites within a 12 month pilot study the amount of miles travelled by patients was reduced by 8,979 miles with an estimated reduction in drive time of 236 hours. At the individual practice level all sites saw an increase in adherence to CAD/IVD and CHF appropriate care metrics. Eastridge Family Medicine saw an increase from $68.7 \%$ adherence in CAD/IVD metrics to $79.4 \%$ and an increase from $84 \%$ to 95\% in CHF related metrics. Bessemer City Family Medicine also saw positive impact on their scores increasing from 79\% to 93\% and 95\% to 99.4\% in CAD/IVD and CHF metric. Riverbend Family Practice saw gains in their appropriate care scores as well increasing from $77 \%$ to $88 \%$ in CAD/ IVD adherence and 94\% to 99\% in CHF. Over the past year we have been able to prove this program is a success. We have made positive impacts on all of the metrics that were set forth at the beginning of the pilot study surrounding access, patient satisfaction, population health management, and reducing financial burden to our patient population. Some refinement is still to be had in data collection specifically surrounding patient satisfaction with the inherent problems connected to face to face surveying of patients. As an organization however we have come to learn that there are definite positives to leveraging technology and expanding our ability to provide care for patients in a more convenient fashion. Using the knowledge gained here we are working to extend this program to other sites where it makes geographic and financial sense and our goal is double the number of live sites by the end of 2015 .

\section{REFERENCES}

1. Porter, M., \& Lee, T. (2013, October 1). The Strategy That Will Fix Healthcare. Retrieved November 1, 2013.

\section{Objectives}

1. Illustrate how the use of telemedicine can enhance the patient experience in non-traditional care models

2. Identify important factors in site selection for potential host practices

3. Evaluate program outcomes and explore potential opportunities for development
MONDAY, MAY 16, 2016

4:15 PM-5:15 PM

Monday, May 16, 2016

\section{Session 44 \\ Session Title: KNOW THE DIFFERENCE BETWEEN REIMBURSEMENT AND GETTING PAID - NEW REVENUE MODELS FOR VIRTUAL CARE}

PRESENTER: Nina M. Antoniotti, RN, MBA, PhD, Executive Director of Telehealth

SIU Health Care, Springfield, IL

Understanding the new quality-based payment models and how virtual care adds value to such models is often a mystery to the clinician and administrators of virtual care programs. What exactly is a shared savings model, a value-based purchasing model, and how does virtual care add to net earnings at the end of the day is often an un-asked question, let alone an unanswered one! This presentation begins with an overview of today's payment models for health care including the two payment options for accountable care, private health plan shared savings models, and direct contracting with self-insured employers, TPAs, and some commercial and indemnity health plans. Understanding how quality drives cost is imperative for the virtual care administrator and clinician who are attempting to use virtual care to lower cost, improve access and quality, while improving the patient experience. The presentation reviews several programmatic virtual care options used in a multi-specialty clinic environment to help improve revenue streams from non-fee-for-service revenue streams. Financial metrics are discussed with relevance to comparisons of cost pre-and post-use of virtual care, as well as how to integrate financial metrics into contracting with payers for quality-based payments. The use of risk contracting is ever increasing in quality-based payment models and the virtual care organization must understand how risk contracting and virtual care strategies work together. A full explanation of risk contracting and how to weave virtual care into the discussion and terms is included in this presentation. Objectives

1. Understand the differences in revenue models in the current health care paradigms

2. Demonstrate how virtual care adds value to quality-based reimbursement models

3. State how to develop a fiscally meaningful discussion with the executive team on using virtual care in shared savings revenue models

\section{Room M100 DC}

\section{TUESDAY, MAY 17, 2016}

10:00 AM-12:00 PM Tuesday, May 17, 2016

\section{Session 85 Session Title: WORKSHOP}

WORKSHOP: HOW TO GET YOUR WORK PUBLISHED

MODERATOR: Terry Rabinowitz, MD, DDS, Professor of Psychiatry and Family Medicine

University of Vermont College of Medicine, Burlington, VT

PRESENTER: Elizabeth Brooks, PhD, Assistant Professor University of Colorado Denver, Aurora, CO

PRESENTER: Carolyn Turvey, PhD, Professor, Research Health Scientist University of Iowa, Iowa City VA Medical Center, Iowa City, IA 
PRESENTER: Charles R. Doarn, MBA, FATA Research Professor, Family and Community Medicine and Director, Telemedicine Program, Co-Editor-inChief, Telemedicine and eHealth Journal

University of Cincinnati, Cincinnati, $\mathrm{OH}$

PRESENTER: Ronald C. Merrell, MD, FATA Professor of Surgery, Co-Editor-in-Chief, Telemedicine and eHealth Journal

Virginia Commonwealth University, Mentone, $A L$

PRESENTER: Elizabeth Krupinski, PhD, FSPIE, FSIIM, FATA, Professor and Vice Chair of Research

Department of Radiology and Imaging Services, Emory University, Atlanta, GA

The importance of sharing professional knowledge and experiences of practitioners are critical to advancing expertise, research and best practices in any field or discipline. Yet, barriers to getting started in academic and professional publishing exist and stand in the way especially for new or junior researchers and academics. In this workshop, attendees will learn from the experts and gain practical insights and the tools needed to get beyond these challenges and get their work published.

\section{REFERENCES}

1. Brooks E, Turvey C, Augusterfer EF. Provider Barriers to Telemental Health: Obstacles Overcome, Obstacles Remaining. Telemed E Health 2013; 19(6):433-37.

2. Hilty D, Yellowlees PM, Parrish MB, Chan S. Telepsychiatry: Effective, EvidenceBased, and at a Tipping Point in Health Care Delivery? Psychiatr Clin North Am 2015; 38(3):559-92.

3. Menon PR, Stapleton RD, McVeigh U, Rabinowitz T. Telemedicine as a Tool to Provide Family Conferences and Palliative Care Consultations in Critically III Patients at Rural Health Care Institutions: A Pilot Study. Am J Hosp Palliat Med 2015; 32(4):448-453.

\section{Objectives}

1. Describe the importance, value and role of scholarly publications for personal and professional growth, and to foster continuous improvement and quality assurance efforts by individuals and organizations

2. Explain how to manage the manuscript development and publication process and to deal with feedback at each stage

3. Utilize new and existing data effectively to support the main premise or focus of the practitioner research efforts

\section{TUESDAY, MAY 17, 2016}

11:00 AM-12:00 PM

Tuesday, May 17, 2016

\section{Session 52}

\section{Session Title: WHERE THE RUBBER MEETS THE ROAD: TELEHEALTH CODING, COVERAGE, AND REIMBURSEMENT}

MODERATOR: Cybil Gregory Roehrenbeck, JD, Counsel Polsinelli PC, Washington, DC

PRESENTER: Jay Ahlman, Vice President, Coding and Reimbursement American Medical Association, Chicago, IL

PRESENTER: Terrence Lewis, JD, Senior Associate Counsel University of Pittsburgh Medical Center, Pittsburgh, PA

PRESENTER: Natasa Sokolovich, JD, MSHCPM, Executive Director, Telehealth University of Pittsburgh Medical Center, Pittsburgh, PA

In this course, participants will discuss current efforts to identify and effect solutions for coding, coverage, and reimbursement of telehealth services. With respect to coding, the panel will offer insight into the Current Procedural Terminology $(\mathrm{CPT} \circledast)$ process, educate participants about the AMA's formation of a CPT® Telehealth Services Workgroup, and identify opportunities for stakeholders to recommend solutions for the reporting of telehealth services. The panel will also examine the related issues of reimbursement and coverage for telehealth services. Panelists will offer policy, legal, and business perspectives, allowing for a multi-dimensional discussion that offers attendees a $360^{\circ}$ picture of the state of telehealth coding, coverage, and reimbursement.

\section{REFERENCES}

1. Lewis T, Sandy II EA. eHealth, Women's Health and the Legal Framework for a Successful Clinical Program: A View from Across the Atlantic. International Society of Telemedicine and E-Health Global Journal (2015).

2. Roehrenbeck C. E-Health Industry Overview," E-Health, Privacy, \& Security Law, Bloomberg/BNA (2015).

\section{Objectives}

1. Identify and explore key issues concerning telehealth coding, coverage, and reimbursement

2. Educate participants on CPT® code submission and development processes and specific efforts related to coding for telehealth

3. Offer practical suggestions and solutions for industry participants to engage in advocacy and the development of telehealth coding, coverage, and reimbursement

\section{TUESDAY, MAY 17, 2016}

1:15 PM-2:15 PM Tuesday, May 17, 2016

\section{Session 60}

\section{Session Title: PLANNING AND DEVELOPING A MULTI-STAKEHOLDER RURAL AND URBAN TELEHEALTH NETWORK}

MODERATOR: David P. Mortimer, MDiv, Director, Innovation Institute Hospital Sisters of St. Francis Foundation, Hospital Sisters Health System, Springfield, IL

PRESENTER: Gurpreet S. Mander, MD, MBA, CPE, FAAP, MA, FACC, Chief Medical Officer

HSHS St. John's Hospital, Springfield, IL

PRESENTER: Mark R. Stampehl, MD, MA, FACC, Medical Director of Heart Failure

HSHS St. John's Hospital, Springfield, IL

PRESENTER: Chris Schmidt, RN, BSN, Regional Stroke and Telemedicine Nurse Coordinator HSHS St. John's Hospital, Springfield, IL

Background: Healthcare in the United States is experiencing a paradigm shift from a fee-for-service model to a value-based population health model, and from volume to value. Telehealth is paramount in advancing this shift, particularly in rural areas. To further the three-part aim of better health, better healthcare, and lower costs, and to increase rural access to care, health systems must explore new models to proactively plan and develop telehealth networks. Innovative network models are critical to collaborating with diverse and nonintegrated hospitals that may be outside of one's own health system. The creation of a cohesive network from non-integrated stakeholders requires a resolution of the problem of the commons. Elinor Ostrom, in Governing the Commons: The Evolution of Institutions for Collective Action, described this process: "The central question ...is how a group of principals who are in an interdependent situation can organize and govern themselves to obtain continuing joint benefits when all face temptations to free-ride, shirk, or otherwise act opportunistically" (Cambridge University Press, 1990). 
Intent: This presentation will detail the planning, development and design strategies of Illinois Telehealth Network (ITN), a collaborative "consortium network model." This network is comprised of 21 rural and urban hospital stakeholders in Illinois. ITN includes both telehealth and telemedicine services to provide:

1. Acute care telemedicine decision making and evaluation to serve patients in underserved rural emergency rooms (a program that began in 2010);

2. Telehealth remote monitoring for high risk post-acute heart failure patients (a post-acute program that began 15 years ago and is now being expanded in outpatient settings).

The creation, planning and development of this model requires collective action to be conducted by the resource users themselves. The session will explore the use of this innovative model to:

1. Conduct a provider needs assessment to identify critical needs for services that may be provided via telemedicine and telehealth;

2. Create and deploy a strategic and financial plan;

3. Develop an operational plan with focused action and results monitoring.

This presentation will illustrate how this network model helps identify and meet rural hospital needs, uses shared clinical protocols to achieve telemedicine and telehealth efficiencies, builds capacity by expanding access to specialists in underserved rural areas, coordinates care, improves quality, and strengthens the rural healthcare system.

\section{REFERENCES}

1. HRSA. "The Network Guide: A technical assistance resource developed by the Health Resources and Services Administration (HRSA) and the National Association of Community Health Centers (NACHC). Available at: http:// www.hrsa.gov/healthit/networkguide/networkguide.pdf (2012).

2. Komaramy JK, et al. Partnering Urban Academic Medical Centers And Rural Primary Care Clinicians To Provide Complex Chronic Disease Care. Health Affairs 2011; 30(6):1176-184.

3. Ostrom, E. (2008). Governing the Commons. Cambridge, NY: Cambridge University Press.

4. Verhaegh, K., MacNeil-Vroomen, J., Eslami, S., Geerlings, S., de Rooij, S., et.al. Transitional care interventions prevent hospital readmissions for adults with chronic illnesses. Health Affairs 2014; 33(9):1531-539.

\section{Objectives}

1. Understand the planning and operational deployment of a telestroke and telehealth remote monitoring program in a setting with diverse stakeholders

2. Gain insights into the planning and development of an innovative consortium network model to create a multi-stakeholder telehealth network to save lives and improve patient outcomes

3. Acquire an understanding of care integration planning that brings rural and urban telemedicine and telehealth remote monitoring stakeholders together to create economies of scale and reduce costs

\section{TUESDAY, MAY 17, 2016}

3:00 PM-4:00 PM Tuesday, May 17, 2016

\section{Session 68 Session Title: ATA PRACTICE GUIDELINES: HOW CAN THEY BE USED EFFECTIVELY?}

MODERATOR: Elizabeth Krupinski, PhD, FSPIE, FSIIM, FATA, Professor and Vice Chair of Research

Department of Radiology and Imaging Services, Emory University, Atlanta, GA
PRESENTER: Alexis Gilroy, JD, Partner

Jones Day, Washington DC

PRESENTER: Fredda Kermes, RN, BSN, MHI, Director of Professional Development/Telemedicine Coordinator

Summit Healthcare Regional Medical Center, Show Low, AZ

ATA has been producing practice guidelines for telemedicine since 1999, with 14 freely available for download from the ATA website. In the past, Committee members have presented results on surveys about how those involved in telemedicine are using them and how often they are downloaded. In this session, speakers will discuss four key ways in which practice guidelines are used to promote telemedicine: why having practice guidelines is important from a legal or regulatory perspective; how guidelines can be effectively integrated into clinical practice; how they can be used to start a telemedicine program and convince stakeholders to participate; and how they can be used in telemedicine research and program evaluation. Attendees will also hear about recent guideline developments from the ATA Practice Guidelines Committee.

\section{Objectives}

1. Gain insight about ways practice guidelines promote quality care and improve patient outcomes

2. Understand how to utilize practice guidelines and integrate guidelines into clinical practice

3. Learn about active guidelines projects underway at ATA

\section{TUESDAY, MAY 17, 2016}

4:15 PM-5:15 PM Tuesday, May 17, 2016

\section{Session 76 Session Title: TELEMEDICINE IN EDUCATION}

MODERATOR: Ellen R. Cohn, PhD, CCC-SLP, Professor, Department of Communication Science and Disorders

School of Health and Rehabilitation Sciences (SHRS), University of Pittsburgh, Pittsburgh, PA

INNOVATIVE INTERPROFESSIONAL TELEMEDICINE EDUCATION: PREPARING FUTURE PROVIDERS FOR COLLABORATIVE PRACTICE

PRESENTER: Sue Chase-Cantarini, DNP RN, Assistant Professor University of Utah, Salt Lake City, UT

Recent and proposed changes in healthcare delivery necessitate interprofessional collaborative practice to improve healthcare safety and quality. Concurrently, there is a substantial increase in implementing technology to effectively manage and optimize the health of individuals with multiple chronic conditions, especially those living in rural communities. In 2013, the University of Utah College of Nursing was awarded a three year Health Resources and Services Administration Advanced Nursing Education training grant to address these initiatives. The primary goal of the grant was to help alleviate the disparities in healthcare access for residents of rural regions of Utah who lack appropriate and necessary healthcare providers and services. The unique and novel dimension to this project was the use of telehealth video conferencing activities to connect healthcare experts from multiple disciplines and specialties from one central hub to the rural practitioners who are managing patients with multiple chronic conditions.

The overarching goal is to promote and facilitate interprofessional collaboration and clinical teamwork among graduate students representing diverse health professions through an innovative education model. The design and implementation of this model was embedded in 


\section{ORAL PRESENTATIONS ABSTRACTS}

current health science curricula in which students from a variety of disciplines participated in a structured and shared experience of resolving clinical issues encountered in the care of complex patients in rural communities. General aims are three-fold: (1) introduce students to best practices of telemedicine, (2) promote an understanding of interprofessional practice competencies through tele-video conferencing (TVC) and, (3) improving the healthcare of rural patients who lack specialty medical and allied healthcare services through the implementation of telemedicine technology.

There are two distinct, yet complementary phases of our innovative educational program. Both phases include graduate students from various professions and involved in the program's collaborative partnership: medicine, health, nursing, pharmacy and social work. Multiple levels of assessment and evaluation are tied to each phase of the program. During the first phase, we developed a series of self-paced interactive online modules followed by a simulation exercise where students attend using secure TVC software. The modules evolved into a credit bearing course that was integrated in our Interprofessional Education Program. The first three modules introduced key aspects of telemedicine standards applicable to clinical settings, interprofessional practice competencies, and healthcare needs of patients living in rural communities. A pre- and post-survey for each module assessed student knowledge of the content provided. A fourth module comprised a patient chart, student schedule, timeline, provider scripts and log-in instructions. The actual simulation sessions had teams of students collaborating with each other and providing consultation/recommendations for a patient with multiple chronic conditions. Best practices of simulation and debriefing were implemented to promote successful student experiences and outcomes. To date, over 200 students have participated with another 200 planned for the current academic year. Student's attitudes and beliefs towards interprofessional practice and use of telemedicine technology were collected via survey instruments prior to and at the completion of the course. The survey data indicated an overwhelmingly positive response and confirmed that an interactive online module followed by a simulated telemedicine scenario was an effective learning strategy. Students were able to increase their understanding of interprofessional roles and acknowledge the unique contributions of various disciplines in managing patients with multiple chronic diseases. Several students suggested that the course be offered to all health sciences students and saw the value of using telemedicine technology in future practice.

The second phase began in 2015, when we implemented Clinical Immersion (CI) experiences for students to join actual encounters with patient consultation through TVC. Interprofessional care management cases were presented by Doctor Nurse Practice distance students (and their preceptor) to teams of multidisciplinary colleagues. Depending on the case, students from various professions attended with faculty preceptors to provide mentorship in professional consultation. CIs are conducted in partnership with Project ECHO (Extension Community Healthcare Outcomes) and will continue each semester. All individuals participating in the CIs engage in a debriefing after the session in addition to completing evaluation tools (e.g., self-evaluations, preceptor/student performance feedback and reflection assignments.) The data has indicated important outcomes: 1) it is a positive experience for students and exceeded their expectations, 2) improved knowledge, 3) and desire to use telemedicine technology in the future. Many see themselves future leaders of this technology in their clinical practice. Additional plans to extend the educational program are: 1) to provide video-taped session will be used as relevant future case studies in interprofessional classrooms; 2) Transportable Exam Stations are being introduced and used by students in physical assessment, diagnostic reasoning and practicum courses, and 3) to develop a continuing education course and building scenarios to include additional disciplines.
Survey data has been collected from all phases of the grant project and preliminary results will be presented and implications for education and health practice will be discussed.

\section{REFERENCES}

1. Bulik RJ, Shokar GS. Integrating telemedicine instruction into the curriculum: expanding student perspectives of the scope of clinical practice. J Telemed Telecare 2010; 16(7):355-58

2. Conde JG, et al. Telehealth innovations in health education and training. Telemedicine and e-Health 2010; 16(1):103-106.

3. Gray DC, Rutledge CM. Using new communication technologies: An educational strategy fostering collaboration and telehealth skills in nurse practitioners. J Nurs Pract 2014; 10(10):840-44.

4. Weinstein, R. S, et al. Technologies for interprofessional education: The interprofessional education-distributed "e-Classroom-of-the-Future". J Allied Health 2010; 39(3)Pt 2:238-245.

\section{Objectives}

1. Describe benefits of telemedicine education in academic based health science education

2. Identify strategies to incorporate telemedicine technology into university-based multidisciplinary health science curricula

3. Explore innovative and effective approaches to combine best practices of telemedicine and interprofessional competencies in education and practice settings

VIRTUAL CARE OPTIONS FOR COMMUNITY COLLEGES - NAVIGATING A POPULATION SPECIFIC HEALTH INITIATIVE FOR COMMUNITY COLLEGE STUDENTS AND EMPLOYEES

PRESENTER: Nina M. Antoniotti, RN, MBA, PhD, Executive Director of Telehealth and Clinical Outreach

SIU Health Care, Springfield, IL

In the United States, community colleges are a gateway to opportunity for many students, offering low tuition rates for two to four year college degrees. There are approximately 12 million community college students, with an average age of 28 . Fifty seven percent of community college students are female, and $49 \%$ of students are in minority groups. With their open-access model and low tuition rates, community colleges are a gateway to opportunity. Nearly half of all undergraduates in the United States attend community college. More than half of community college students enroll part time. In Illinois, most community colleges are in areas where access to care is limited and most students are under or not insured. Meeting the needs of student populations who have unique vulnerabilities to population health issues is difficult when access to primary care is limited, and specialty care in nonexistent. This presentation outlines the approach used by SIU Health Care to meet the needs of a regional community college with 7,000 students and 1,000 employees. The approach used to provide access to care used a blended approach of traditional clinic to clinic telehealth and direct-to-consumer options for after hours, urgent care. Most direct-to-consumer models offer cash pay options for convenience and privacy. But how well do these economic models work in a community college population? Does the same business model work? Does the same healthcare delivery model work? This presentation covers all aspects of key considerations when developing a model of virtual care for community colleges. Community colleges are one of the last untapped areas where telehealth can play a key role in shaping and improving the health of populations. This detailed presentation covers the best approach to success for TeleHealth and community colleges. 
1. American Association of Community Colleges. 2014 FACT Sheet. Washington DC

2. Katz DS, Davison K. Community college student mental health. Community College Rev 2014; 42(4):307-26.

Objectives1. Identify the unique healthcare needs of a community college population2. Understand virtual care and direct to consumer strategies that might help address the unique healthcare needs of community college populations3. Demonstrate the key steps in developing a comprehensive virtual care and direct to consumer strategy for working with community colleges

\section{Track: Policy and Legal Room 101 HG}

\section{SUNDAY, MAY 15, 2016}

12:00 PM-1:00 PM

Sunday, May 15, 2016

\section{Session 8}

\section{Session Title: BUILDING US TO CHINA INTERNATIONAL TELEMEDICINE ARRANGEMENTS}

MODERATOR: Nathaniel Lacktman, JD, CCEP, Partner

Foley and Lardner LLP, Tampa, FL

PRESENTER: Nathaniel Lacktman, JD, CCEP, Partner Foley and Lardner LLP, Tampa, FL

PRESENTER: Terrence Lewis, JD, Senior Associate Counsel

University of Pittsburgh Medical Center, Pittsburgh, PA

PRESENTER: Peter Kung, BA, MS, MBA, System Vice President, Virtual Health

SCL Health, Broomfield, CO

U.S. hospitals and providers, seeking to share their clinical expertise and broaden their footprint/patient base, are looking abroad for telemedicine opportunities, particularly in China, a country with a robust economy and limited healthcare access.

U.S. hospitals want to explore telemedicine arrangements with China medical institutions, but are fumbling in the dark, trying to figure it out. The legal issues, business structures and cultural differences are significant, but arrangements can be created for international bridges of healthcare.

This session, hosted by three speakers from three different systems, each of whom has built U.S. to China telemedicine arrangements, will educate attendees on the legal and regulatory issues plus business and economic considerations when creating an arrangement between a U.S. hospital and a China medical institution.

\section{REFERENCES}

1. China Technical Guideline for Telemedicine Information System Construction (2014).

2. China Opinions of the National Health and Family Planning Commission Regarding Promoting Medical Institutions' Telemedicine Services (2014).

3. China Administrative Measures for Internet-based Medical and Health Information Services (2009).

4. China Notice on Strengthening the Administration of Telemedicine Consultation (1999).

\section{Objectives}

1. Understand the business case and decision points for U.S. to China telemedicine arrangements
2. Learn legal and regulatory steps to create a viable, compliant contractual arrangement

3. Explore variants to these models and obtain useful handouts

\section{SUNDAY, MAY 15, 2016}

\section{Session 16}

Session Title: BOOTS ON THE GROUND: A PRACTICAL REVIEW OF RISKS AND REGULATORY PITFALLS IN TELEHEALTH ENGAGEMENT

PRESENTER: Sidney Welch, JD, Partner

Kilpatrick Towsend \& Stockton LLP, Atlanta, GA

From state regulations to vendor negotiations, there are a number of steps along the way of telehealth engagement that can derail the most promising of clinical models. This session will provide the practical insights of an attorney who counsels a diverse range of clients who have sought to engage in telehealth innovation and deployment. A discussion of how to assess risk in telehealth ventures; legislative and regulatory developments on the horizon; key contractual considerations; and business elements will help inform attendees' own telehealth projects and goals. Among other takeaways, attendees will be provided a checklist of key contractual considerations when working on telehealth partnerships.

\section{REFERENCES}

1. Looney, K., Schaff, M., Welch, S. "Telemedicine and the Impediments Facing Effective Implementation," Physician Organizations, American Health Lawyers Association (June 2015)

2. C. Roehrenbeck, "E-Health Industry Overview," E-Health, Privacy, \& Security Law, Bloomberg/BNA (2015).

Objectives

1. Offer attendees a practical checklist of contractual issues to consider

2. Provide examples of contractual or regulatory hurdles and methods to overcome those challenges

3. Educate attendees as to meaningful risk profiles vs. issues that can be easily mediated

MONDAY, MAY 16, 2016

8:00 AM-8:45 AM

Monday, May 16, 2016

\section{Session 24}

Session Title: REGULATORY TOPICS FOR M-HEALTH

MODERATOR: Gary Capistrant, Chief Policy Officer

American Telemedicine Association

REGULATORY CONSIDERATIONS FOR CONNECTED MOBILE DIAGNOSTICS

PRESENTER: Douglas Foster, MBA, Chief Executive Officer

DigiSight Technologies, San Francisco, CA

The development of low-cost mobile diagnostics will lead to a paradigm shift in how we think about medical devices, and more importantly, medical data. We no longer need bigger and more complex systems that cost more money to generate better outcomes for patients or to create more efficient models for providing care. Instead, our healthcare system needs diagnostic 
tools that are "good enough" while simultaneously providing more information than ever before on patient wellbeing.

DigiSight Technologies develops connected mobile tools to provide physicians with deeper insights on patient health by generating medical data and transmitting, storing and displaying this data on a cloud-based platform that can be accessed by physicians. These mobile tools, however, are subject to both medical device regulations as well as data regulations for protected health information (PHI).

DigiSight will share key lessons and insights from its own experience with these regulatory requirements in the context of developing its mobile tools, which include a dozen standard ophthalmic tests. Specifically, we will focus on DigiSight's cloud-based Paxos platform, which includes Paxos Checkup, a vision testing application, and Paxos Scope, a mobile ophthalmic camera utilizing a hardware adapter for smartphones.

In this presentation, DigiSight will use its own experiences as a framework to provide an overview of the following regulatory considerations for connected mobile diagnostics:

Device regulation

The FDA regulates medical devices, and mobile diagnostics are no exception. Similar to standard medical devices, these diagnostics are classified into one of three categories (Class I, Class II, or Class III) based on the device's associated level of risk and diagnostic claims. However, regulations and guidelines that pertain specifically to mobile diagnostics are still being defined and interpreted; therefore, they are not as well understood as traditional medical devices. As a result, the regulation of mobile diagnostics is often highly complex.

Data security

Connected mobile diagnostics that are used in the United States generate and transmit electronic PHI that must be managed in compliance with HIPAA regulations. HIPAA outlines processes, procedures and technical requirements for PHI management. These technical requirements include guidelines for the collection, transmission and storage of patient information.

International data management

The portability of mobile diagnostics makes it easy to transfer these tools over international borders, especially diagnostics that lack a hardware component.

However, regulations differ across international borders, both for devices and for the transmission and storage of patient health information, and it is important for both developers and users of mobile diagnostics to recognize and understand these requirements prior to using these tools internationally.

\section{Objectives}

1. Apply knowledge of connected mobile diagnostic regulations to development and use of these technologies

2. Navigate FDA classification for mobile diagnostic tools

3. Evaluate requirements for the generation, transmission and storage of patient health information, both in the US and abroad

MONDAY, MAY 16, 2016

11:00 AM-12:00 PM

Monday, May 16, 2016

\section{Session 32 \\ Session Title: ARE YOU READY FOR DIGITAL HEALTH PATENT DISPUTES?}

PRESENTER: Doug Pearson, PhD, JD, BS, Attorney

Jones Day, Washington, DC

PRESENTER: Mark Paulson, JD, BA, Attorney

Jones Day, Washington, DC

With exponential growth occurring in the availability of telemedicine and other digital health products and services, patent disputes are sure to erupt and, in fact, are already starting. These disputes are occurring on the heels of the America Invents Act, which became effective in 2012, and which gave birth to post-grant proceedings - inter partes reviews (IPRs), post-grant reviews (PGRs), and covered business method reviews (CBMs) - which permit third parties to challenge the validity of granted patents before the Patent Trial and Appeal Board (PTAB) of the U.S. Patent and Trademark Office (USPTO). IPRs and CBMs have become wildly popular and can now be expected to be key counterparts of many, if not the majority of, district court patent suits. With the ability to challenge the validity of patents in both federal courts and the USPTO, the need for patent owners to acquire strong patents, and the need for challengers to understand likely areas of weakness, are more important than ever. The terrain is particularly treacherous for computer-implemented inventions (also called software inventions) in light of several recent court decisions addressing, among other things, i) subject matter eligible for patenting, ii) how direct infringement may or may not be found in the aggregate acts multiple different entities, iii) the effect on patent validity/scope of unintended "means" interpretations of patent claims, and iv) how functional claiming common in telemedicine/digital-health patents may run afoul of the requirement that patent claims recite the scope of the patent right with "reasonable certainty." While the challenges facing digital health patent holders can be significant given recent court rulings addressing these issues, they can be overcome in many instances with an eye toward good claim drafting for new and pending patent applications and through possible use of the "reissue" process at the USPTO to correct patent claims before asserting the patent in litigation. This tutorial will assist participants in understanding these areas of potential weakness for digital-health patents, strengthening patent portfolios in light of these issues, challenging the patents of others in light of these issues, how the new post-grant proceedings at the USPTO are transforming patent litigation, and how patent applicants can draft patent applications to increase their chances of surviving a post-grant challenge at the USPTO.

\section{REFERENCES}

1. Pearson, D., et al., Jones Day, Digital Health Law Update 2015; 1(4), Washington, DC, Jones Day. http://www.jonesday.com/digital-health-law-update-vol-1issue-4-08-07-2015

\section{Objectives}

1. Recognize areas of potential weakness of telemedicine/digital-health patents

2. Apply that recognition to understand how to strengthen one's own patents and patent applications and how to challenge the patents of others

3. Develop strategies for creating strong digital-health patent portfolios given an understanding of areas of potential weakness and given the nature of post-grant challenges at the USPTO

\section{Roundtable 8 Room 211 AB}

\section{MONDAY, MAY 16, 2016}

1:15 PM-2:15 PM

Monday, May 16, 2016

\section{Session 84}

\section{Session Title: ROUNDTABLE}

TELEMEDICINE START-UPS: WHAT YOU NEED TO KNOW

PRESENTER: Nathaniel Lacktman, JD, CCEP, Partner

Foley and Lardner LLP, Tampa, FL 
In the telemedicine space, there are so many great people with the next "big idea" in health innovation. But even the best ideas need a business framework in order to launch. This Roundtable session will start with a topical outline and checklist of everything a telemedicine start-up needs to have from a businesslegal perspective. This includes, for example, corporate structure, contracting, privacy practices, NDAs, forms, terms of use, advisory board creation, etc. Then participants will have open Q\&A to have any of their start-up questions answered on the spot. The goal by the end of the session is for the attendees to feel confident they have a solid roadmap and checklist of the documents they need to launch their telemedicine start-up.

Many entrepreneurs and start-ups feel confident in their skills and experience, but overwhelmed, confused (or even intimidated) by all the variations across state laws and all the fraud $\&$ abuse rules in the healthcare industry. This session is designed to give a practical, interactive crash course in what a telemedicine start-up needs to have from a business-legal perspective.

\section{Objectives}

1. Identify the set of documents and legal decisions a telemedicine startup requires

2. Learn the legal issues through practical examples

3. Discuss and answer the pressing questions of telemedicine start-up entrepreneurs

\section{MONDAY, MAY 16, 2016}

4:15 PM-5:15 PM Monday, May 16, 2016

\section{Session 45}

\section{Session Title: ACOS AND TELEHEALTH - USING ACO WAIVERS TO GET HEALTH IT INTO THE HANDS OF DOCTORS}

PRESENTER: John M. Kirsner, JD, Partner

Jones Day, Columbus, $\mathrm{OH}$

PRESENTER: Michelle Johnson Tidjani, JD, Counsel

Cleveland Clinic Foundation, Cleveland, $\mathrm{OH}$

The purpose of this session will be to examine the legal and regulatory situation currently in the USA regarding the ability to take advantage of governmentally promulgated waivers from the healthcare regulatory laws relating to accountable care organizations and related ventures, such as clinically integrated networks. The presentation will include a detailed overview of the current regulatory scene relative to using waivers, including reference to several "real world" examples of putting waivers into place at specific healthcare organizations. Presenters will discuss how The Cleveland Clinic has considered using the ACO waivers relating to placing outstanding patient care health IT tools into the hands of physicians participating in the CCF ACO. Strategies to allow for the use of innovating health IT in a clinically integrated network setting will also be considered. After attending this panel, attendees should be more familiar with the ACO, with the ability to use an ACO to waive areas of existing law to promote innovative healthcare strategies to bring better, more efficient care to patients, and how to perfect an ACO waiver, even if the ACO is not yet fully up and running. Attendees should also have a good understanding of the "state of play" of what organizations are doing nationally regarding using the ACO waiver to acquire digital health and health IT for their organizations.

Objectives

1. Outline structure of ACO waiver process given CMS requirements

2. Identify unique aspects and opportunities for telehealth within ACO structure

3. Identify positive use cases for ACO structure with respect to digital health
TUESDAY, MAY 17, 2016

11:00 AM-12:00 PM

Tuesday, May 17, 2016

\section{Session 53 \\ Session Title: EXAMINING HOW MEDICAL ETHICS MUST EVOLVE IN TANDEM WITH TELEHEALTH}

MODERATOR: Jennifer Ruschman, ScM, Director, Center for Telehealth Cincinnati Children's Hospital Medical Center, Cincinnnati, $\mathrm{OH}$

PRESENTER: Kathleen Kramer, MAT, Consultant, Center for Telehealth Cincinnati Children's Hospital Medical Center, Cincinnati, $\mathrm{OH}$

PRESENTER: Kelsey Hofacer, MHSA, Consultant, Center for Telehealth Cincinnati Children's Hospital Medical Center, Cincinnati, $\mathrm{OH}$

As healthcare has evolved over the last decade, and there is a greater emphasis placed on quality outcomes, many organizations struggle with providing high quality care across large geographies within a single healthcare institution or system. To combat this challenge, many institutions are leveraging telehealth in innovative capacities. However, as telemedicine continues to disrupt the healthcare delivery system, and there are emerging uses for telehealth applications, this may inadvertently create previously undiscussed ethical considerations.

This presentation will provide a general overview of ethics, and will address how to apply the framework of medical ethics to telemedicine. The presentation will highlight three telemedicine programs at a large pediatric academic medical center, and the ethical considerations of seeing patients in their home, in a suburban medical center, or within their international or rural communities. Specifically the presentation will address privacy and reporting issues that arise when non-home health providers see patients via telemedicine in their homes, how telehealth may inadvertently enable community hospitals to care for patients that have a higher acuity, and how telehealth improves access for international patients, despite their ability or resources to receive ongoing care or best-practice medical therapies. Finally, the presentation will address how one institution has begun to work to mitigate potential ethical issues through provider education, consent, and well-defined clinical practice algorithms.

Objectives

1. Provide a general understanding of bioethics, specifically medical ethics

2. Recognize, identify, and understand how to mitigate ethical issues as they arise within the constructs of telehealth

3. Promote conversations surrounding ethical considerations in telehealth

TUESDAY, MAY 17, 2016

1:15 PM-2:15 PM Tuesday, May 17, 2016

\section{Session 61}

Session Title: WITH EXPANSION COMES COMPETITION: THE ANTITRUST IMPLICATIONS OF AN EVOLVING TELEMEDICINE MARKET

MODERATOR: Alexander Hecht, JD, Vice President of Government Relations

ML Strategies LLC, Washington, DC

PRESENTER: Dionne Lomax, JD, Member

Mintz, Levin, Cohn, Ferris, Glovsky and Popeo, PC, Washington, DC

PRESENTER: Andrew Adair, JD, Government Relations Representative American Academy of Family Physicians, Washington, DC 
Antitrust issues in the Telemedicine space are becoming increasingly important. As telemedicine continues to expand and change how healthcare is delivered, it is critical for industry participants to ensure that they are not violating the antitrust laws as they seek to compete. The DOJ, FTC, and other healthcare authorities are paying close attention to the healthcare industry, including transactions among providers and health insurers. They are also closely scrutinizing the conduct of various industry participants - healthcare service providers, insurers, and even state regulatory bodies that monitor certain aspects of healthcare delivery. This session will discuss the types of antitrust issues that can arise as telemedicine continues to transform the competitive landscape in the industry and provide conference attendees with insight into how the antitrust authorities are likely to view competitive reactions to new market participants. Telemedicine providers will also gain keen insight into the various ways to ensure they steer clear of antitrust violations.

One of the key issues arising as telemedicine continues its entry and expansion into the provision of healthcare services, is the extent to which state medical boards can impose regulations that restrict the ability of telemedicine providers to practice in the state. This issue is front and center in Texas where ongoing litigation is occurring between Teladoc, Inc. (a national telemedicine provider) and the Texas Medical Board (Complaint, Teladoc Inc. v. Texas Medical Board, No. 1-15-CV-343-RP (W.D. Tex. April 29, 2015), ECF No. 1) regarding the Board's issuance of a new regulation which would have effectively prevented the practice of telemedicine medicine in the State. Earlier this year Teladoc and other plaintiffs sued the Board, alleging that the Board's new regulation violates Section 1 of the Sherman Act and the Commerce Clause. This ongoing legal battle involves issues regarding the standards for appropriate telemedicine practice in Texas and is one of the first major cases challenging the actions of a state medical board in the wake of the Supreme Court's decision in North Carolina State Board of Dental Examiners v. Federal Trade Commission, 135 S. Ct. 1101 (2015). In that case, the Supreme Court held that the antitrust laws would apply to - and the state action exemption would not protect - activities of state agencies or boards made up of market participants, absent active state supervision of the Board's challenged conduct.

The rise of telemedicine medicine may also impact how traditional healthcare service providers may react and respond to the entry and expansion of telemedicine. Hospitals, physicians, and various provider associations will need to be particularly careful about the rules, regulations and other changes they seek to limit or restrict the way telemedicine providers may provide healthcare services.

Finally, telemedicine service providers may also need to be mindful of the antitrust laws as they grow and expand. While the remote provision of healthcare services likely has many procompetitive benefits (i.e., improved access to underserved patients, improved quality, and decreased costs), the antitrust agencies want to ensure that telemedicine providers (like any new entrant) is engaged in efficiency-enhancing growth without restricting competition.

\section{REFERENCES}

1. Complaint, Teladoc Inc. v. Texas Medical Board, No. 1-15-CV-343-RP (W.D. Tex. April 29, 2015), ECF No. 1.

2. North Carolina State Board of Dental Examiners v. Federal Trade Commission, 135 S. Ct. 1101 (2015).

\section{Objectives}

1. Understand antitrust enforcement trends in the healthcare industry and how the antitrust laws impact the telemedicine industry

2. Recognize the potential effects certain conduct by telemedicine providers and other market participants may have on competition in the industry and identify conduct that may raise red flags and be of concern to antitrust enforcers

3. Synthesize the changes in federal and state legislation that impacts telemedicine and comprehend the scope of telemedicine services covered by Medicare

\section{TUESDAY, MAY 17, 2016}

3:00 PM-4:00 PM

Tuesday, May 17, 2016

\section{Session 69 \\ Session Title: SCHOOL TELEMEDICINE: MOVING THE LEGISLATIVE NEEDLE}

MODERATOR: Julie C. Hall-Barrow, EdD, Vice President, Virtual Health and Innovation

Children's Health System of Texas, Dallas, TX

PRESENTER: Danielle Wesley, MHA, Senior Director, School Children's Health System of Texas, Dallas, TX

PRESENTER: Stormee Williams, MD, Medical Director, School Programs Children's Health System of Texas, Dallas, TX

PRESENTER: Tamara Perry, BS, Director, Telehealth Operations Children's Health System of Texas, Dallas, TX

Telemedicine adoption rates are continuing to increase at a fast pace but regulators and payors adoption is much slower. There is no single widely accepted standard for private payers as well as many states regulate payments (Medicaid) for telemedicine in no consistent manner. This disparity creates sustainability barriers for many telemedicine programs that are focused on providing access to care to vulnerable populations (Reynolds, C \& Maughan E, 2014).

Children's Health of System of Texas (CHST); a recipient of the 1115 waiver program established a school based telemedicine program to improve access to care and reduce emergency room visits for low-acuity conditions. The robust program consists of 57 school based telemedicine clinics across North Texas. The waiver funding allows for a three-year program that should be sustained at end of program. The barrier to sustainability was payment from Medicaid (the largest patient population in North Texas). Although the school is a billable site for Medicaid, it must be the Primary Care Provider on record. As CHST is not the PCP for all students in the 57 schools, payment was non-existent.

The solution to this problem was to initiate a bill that would allow for any provider to bill in a school based telemedicine clinic. CHST Government Relations and the Telemedicine team developed a strategy to educate key stakeholders about the CHST school based telemedicine program. The bill was passed in the 84th Texas Legislature - HB 1878 Telemedicine in Schools Bill.

The program allows school nurses to connect with providers at Children's Health Pediatric Group for consultations during school hours. Utilizing a live video and audio feed and the use of clinical peripherals, providers can assess and diagnose common illnesses and send prescriptions electronically to the families pharmacy of choice if needed. By expanding the resources available to the school nurse, children in North Texas will be able to avoid urgent and emergency care for low acuity events.

The program is utilizing the waiver funding for the initial start-up and equipment purchases. As we close out our third and final year of funding - the barrier to billing has been eliminated. Our focus will remain on providing quality care through telemedicine for years to come.

\section{REFERENCES}

1. HB 1878; 84th session Texas Legislature. Telemedicine in Schools Bill.

2. Reynolds CA, Maughan ED. Telehealth in the School Setting: An Integrative Review. J Sch Nurs 2015; 31(1):44-53.

\section{Objectives}

1. Demonstrate awareness of influencing legislative constituents

2. Identify key successes and barriers in developing a school-based telemedicine program

3. To describe key strategies for developing acceptance of telemedicine by senior leaders in school districts 


\section{ORAL PRESENTATIONS ABSTRACTS}

\section{TUESDAY, MAY 17, 2016}

\section{4:15 PM-5:15 PM Tuesday, May 17, 2016}

\section{Session 77}

\section{Session Title: DEVELOPING AN ACTION AGENDA} FOR YOUR STATE

MODERATOR: Latoya Thomas, Director, State Policy Resource Center American Telemedicine Association, Washington, DC

PRESENTER: Catherine S. Britain, Executive Director

Telehealth Alliance of Oregon, Medford, OR

PRESENTER: Kofi Jones, Vice President of Government Affairs American Well, Boston, MA

PRESENTER: Nancy L. Kaszak, JD, Director

Illinois Telehealth Initiative, Chicago, IL

PRESENTER: Rachel Mutrux, Senior Program Director of Telehealth Missouri Telehealth Network, Columbia, Missouri

Telemedicine is getting increased attention from state policymakers around the country. They seek to reduce health care delivery problems, contain costs, improve care coordination, and alleviate provider shortages. Many may want to use more telemedicine to achieve these goals. Driving the momentum for telemedicine adoption is the creation of new laws that enhance access to care via telemedicine, and the strengthening of existing policies with greater implications. Patients and health care providers are benefitting from policy improvements to existing parity laws, expanded Medicaid service coverage, and the removal of arbitrary statutory and regulatory barriers. This session will highlight key strategies to develop relationships with lawmakers, build multi-stakeholder consensus, identify telemedicine issues that are appropriate for the lawmaking process, and outline effective approaches for statewide legislative and regulatory policy improvements.

\section{Objectives}

1. Build relationships and partnerships with key lawmakers and multistakeholders and identify telehealth policy areas that can achieve mutual stakeholder agreement

2. Understand the implications of crafting state telemedicine legislation and regulation

3. Leverage key resources including anecdotal and quantitative outcomes to improve state telehealth policies 


\section{ePoster Presentations Abstracts}

\section{The American Telemedicine Association 2016 Annual \\ Meeting and Trade Show}

\section{Track: Direct to Consumer}

\section{SUNDAY, MAY 15, 2016}

4:45 PM-5:10 PM Sunday, May 15, 2016

\section{EPOSTERS: MEET THE AUTHOR IN THE ATA EXPERIENCE ZONE/EXHIBIT HALL}

\author{
P1 TELEMARKETING: IT'S NOT WHAT YOU THINK: A HOW-TO GUIDE \\ FOR PROMOTING DIRECT-TO-CONSUMER TELEHEALTH

\section{PRESENTER:} \\ Olivia C. Boyce, BA, Marketing Manager \\ InSight Telepsychiatry, Marlton, NJ
}

Telehealth is a hot new topic that allows for unprecedented access to care and unmatched flexibility for services and appointments, but many questions still abound about this topic - What is it? How does it work? What's legal? What's appropriate? This presentation will teach providers and health organizations how to successfully market telehealth services to their current clients, potential clients and the wider communities. Attendees will learn how to define their target audience and refine their telehealth messaging accordingly. They will also learn to assess and prioritize potential outreach mediums for promoting telehealth services including digital ads, social media, print media, events, blogging, PR and educational opportunities. By the end of the presentation, providers and health organizations will be well versed in explaining the benefits and conveniences of telehealth to different audiences and have an understanding of the advantages and challenges of marketing telehealth services to a wide audience across multiple mediums.

\section{objectives}

1. Learners will leave the session able to define their target telehealth audiences

2. Learners will leave the session able to adapt their messaging to be successful with different audiences and across different mediums

3. Learners will leave the session able to make informed decisions when selecting marketing mediums for telehealth promotion

\section{Track: Acute Care}

MONDAY, MAY 16, 2016

10:35 AM-11:00 AM

Monday, May 16, 2016

\section{EPOSTERS: MEET THE AUTHOR IN THE ATA EXPERIENCE ZONE/EXHIBIT HALL}

\section{P25 Simulation BASED MOCK CODES IN A TELE-ICU PRACTICE} PRESENTER:

Sarah Bell, RN, MSN, MHA, Nurse Manager - Enhanced Critical Care Mayo Clinic, Rochester, MN
Mayo Clinic's Tele-ICU program involves remote monitoring of ICU patients by a centralized team of Intensivist's, NP/PA's, and critical care RNs. Collaborating via video during a cardiopulmonary arrest can prove to be difficult. Simulation-based mock codes between tele-ICU and bedside staff were implemented to promote team work, provide definition of roles, and increase collaboration between teams.

Educators traveled on-site to take bedside members through the event while nursing education staff were in the monitoring center located in Rochester, MN. Bedside staff were told to respond as they normally would to the event, they were not prepped about the possibility of involving tele-ICU in the event. This was intentional to measure the rate that tele-ICU staff were included by the bedside staff. Monitoring center RNs and Intensivist were not informed that mock codes were taking place so the scenarios could elicit an un-prepared reaction.

Results found that traditional team member positions and roles need modification for interaction with tele-ICU. Closed-loop communication has greater emphasis so remote team members can be effective. Identification of a team leader remains an essential part of resuscitation, however the location of the team leader, whether remote or at the bedside, does not impact their overall ability to lead.

Simulation-based mock codes were instrumental in defining tele-ICU team members' roles in resuscitation events. It also provided the opportunity for bedside staff to identify the value of remote team members during a critical event.

Objectives

1. Define role of remote monitoring and bedside staff during cardiopulmonary arrest

2. Demonstrate the process of performing simulation based mock codes

3. Summarize the benefits of performing simulation based mock codes in a Tele-ICU practice

P26 THE RELIABILITY OF ROBOTIC TELEMEDICINE FOR ASSESSING CRITICALLY ILL PATIENTS WITH THE FULL OUTLINE OF UNRESPONSIVENESS (FOUR) SCORE AND GLASGOW COMA SCALE: TELECOMA

PRESENTER:

Amelia Adcock, MD, Vascular Neurology Fellow

Mayo Clinic, Phoenix, AZ

Introduction: Telemedicine is increasingly utilized in the evaluation of critically ill patients including those with decreased level of consciousness (LOC) or coma. Improving access to providers with neurologic expertise affords earlier triage and directed patient management. However, objective data regarding the reliability of using standardized coma scales traditionally employed at the bedside for remote assessment are largely lacking.

Hypothesis: Bedside and remote assessments of patients with decreased LOC using either the Glasgow Coma Scale (GCS) or Full scale of Unresponsiveness (FOUR) score are equivalent.

Methods: Prospective trial comparing the reliability of bedside and remote coma assessments using GCS or FOUR score clinical evaluation tools and already validated robotic telepresence technology. Total scores of the GCS and FOUR score were compared between bedside and remote physician assessors by paired t-test and Pearson correlation coefficient (PCC). 


\section{ePOSTER PRESENTATIONS ABSTRACTS}

Results: 100 subjects have been enrolled to date. Mean age was 70.8 $(\mathrm{SD}=15.9)$ years and the average exam time took $5.16(\mathrm{SD}=2.04)$ minutes. Mean GCS total score at bedside was $7.5(\mathrm{SD}=3.67)$ vs. exam conducted remotely $7.49(\mathrm{SD}=3.62)$; difference in scores $0.01(\mathrm{SD}=0.39) ; \mathrm{p}=0.80$. Mean FOUR total score mean at bedside was $9.63(\mathrm{SD}=4.76)$ vs remote 9.21 $(\mathrm{SD}=4.74)$; difference in scores $0.40(\mathrm{SD}=2.00) ; \mathrm{p}=0.05$. PCC for GCS was 0.994; $p<0.001$ and for FOUR score $0.912 p<0.001$. 94.6\% of remote providers rated GCS and $89.2 \%$ rated FOUR score as good (4/5) regarding overall satisfaction and ease of use.

Conclusions: We did not detect any significant difference between total bedside and remote GCS and FOUR scores. Furthermore, Pearson Correlation Coefficients between remote and bedside assessments were excellent $(0.99$ (GCS), 0.91 (FOUR). These results provide persuasive evidence that LOC can be accurately assessed using existing in-Touch telemedicine robots and telecoma evaluation could be employed to improve early evaluation of critically ill patients. Practical implications of this data are also favorable as overall, technical satisfaction was high and evaluation was completed in a timely manner. Limitations to this study include those typical with any real world environment; patients' families were sometimes present, potentially affecting assessment, the majority of assessors were neurologists examining patients with neurologic diagnoses, therefore conceivably limiting application to broader populations with LOC.

1. Wijdicks E. et al. FOUR score and Glasgow Coma Scale in predicting outcome of comatose patients: A pooled analysis. Neurology 2011; 77 (1):84-85

2. Fuhrman SA, Lily CM. ICU telemedicine solutions. Clin Chest Med 2015; 36(3):401-7.

3. Teleneurology applications: Report of the telemedicine work group of the American Academy of Neurology. Neurology 2013; 80:670.

4. Prasad K. The Glasgow Coma Scale: a critical appraisal of its clinimetric properties. J Clini Epidemio. 1996; 49: 755-63.

\section{Objectives}

1. Recognize the importance of expanding validated remote assessment tools to ICU patients

2. Demonstrate awareness of acceptable agreement between bedside and remote patient assessments using standardized coma scales

3. Hypothesize on the benefit of early evaluation of neurologically critically ill patients

\section{P27 IMPLEMENTATION OF A RAPID TRANSFER PROCESS FOR STROKE PATIENTS IN NEED OF ENDOVASCULAR REPERFUSION THERAPY \\ PRESENTER: \\ Jason Close, BS, RRT, Program Manager \\ Dignity Health Telemedicine Network, Carmichael, CA}

The Dignity Health Telemedicine Network instituted a TeleStroke program in 2008. As more spoke hospitals adopted this service, and the volume of TeleStroke consults grew, the need for rapid intervention also increased. Patients could be transferred to any facility offering advanced neurological interventional services, and spoke hospitals were left to determine where, how, and when to send these patients. Often, a lack of communication, understanding of the needs, and coordination led to delays in transportation of the patients; which ultimately led to an inability to treat the patient upon arrival at a Primary or Comprehensive Stroke Center. In response, the Dignity Health Telemedicine Network instituted a Rapid Transfer Process to eliminate these delays. Instead of the spoke hospital being responsible for arranging transport, the TeleStroke neurologist, in conjunction with the Dignity Health Transfer Center takes over. The Transfer Center quickly determines which transport provider has the shortest estimated time of arrival to the spoke hospital, to pick up the patient. They coordinate immediate dispatch of the transport team, even if the decision to transfer has not yet been made. In addition they coordinate an accepting physician, available bed, and clinical reporting. Through the adoption of this method, a significant decrease in transport times has been observed. Patients at remote hospitals are able to receive advanced stroke care, within an appropriate time frame.

1. Rishi G, Zimmermann S, Connelly K, Kommor C. (n.d.). Developing a Collaborative Acute Stroke Network. Retrieved September 11, 2015, from http://my.americanheart.org/idc/groups/ahamah-public/@wcm/ @sop/@scon/documents/downloadable/ucm_448951.pdf

2. Rogers D. (n.d.). Stroke Center Enacts Regional Rapid-Transfer System. Retrieved September 11, 2015, from https://med.stanford.edu/news/allnews/2009/05/stroke-center-enacts-regional-rapid-transfer-system.html

\section{Objectives}

1. Identify the negative effects of delays in patient transport, for patients in need of hyper-acute advanced clinical procedures

2. Discuss the Rapid Transfer Process and its positive implications on patient transport and clinical care.

3. Explain the procedure for implementing a Rapid Transfer Process

\section{P28 EAST MEets WeST: CASE STUDIES IN TELE-ICU}

\section{PRESENTER:}

Teresa Rincon, RN, BSN, CCRN-E, FCCM, eICU Operations Director UMass Memorial Medical Center, Worcester, MA

Understanding the costs and benefits of establishing a Telehealth intensive care unit (Tele-ICU) program is important to defining the role of this evolving technology in promoting more efficient and safer adult critical care. Tele-ICU teams augment care delivery using telecommunications, health information systems, and hardware tools to monitor and assess as well as use surveillance and information gathering methods to promote appropriate therapeutic interventions for critically ill and injured patients (Goran, 2010; C. Lilly \& Thomas, 2010). Tele-ICU teams are generally staffed with experienced critical care nurses, intensivist physicians, critical care trained advanced practice providers and other disciplines that allow expert knowledge to be disseminated to geographically-dispersed and clinically-diverse hospitals (ATA TeleICU Practice Guidelines Work Group, 2014). A case study approach of the first Tele-ICU on the west coast of the US (implemented in 2003) and an academic center Tele-ICU on the east coast (implemented in 2006) will be used to highlight lessons learned as well as financial and clinical outcomes.

Case One

In 2004, nurses in the west coast Tele-ICU started screening patients remotely for severe sepsis for multiple ICUs across one geographical area known as the Sacramento-Sierra region (Rincon, 2012; Rincon, Bourke, \& Seiver, 2011). The west coast Tele-ICU nurses used a paper form downloaded from the Surviving Sepsis Campaign website to develop an online sepsis screening and checklist-to-care tool. These nurses were instrumental in translating knowledge into evidence (knowledge translators), collecting data related to the severe sepsis screening criteria (knowledge acquisition), and using data to develop and refine a sepsis alert known as the sepsis prompt (knowledge creation). Screening patients for severe sepsis is a resource intensive process that requires high-level cognitive processing. There are no tests or biomarkers to aid in prognostic and diagnostic efforts. Using experts (knowledge translators) and designing sophisticated, deterministic algorithms built into health information systems (alerts) were needed to support these efforts.

Expert nurses working in a controlled environment with a specific role to observe and respond to a sepsis alert was an effective and efficient approach to managing a complex sepsis screening process. Much like Intelligence, Surveillance and Reconnaissance (ISR) teams disseminate knowledge to support better decision-making and shape operations, Tele-ICU nurses performed an important role in knowledge translation, acquisition, and creation in order to 


\section{ePOSTER PRESENTATIONS ABSTRACTS}

satisfy the strategic, operational and tactical needs of a large healthcare organization. Several peer reviewed manuscripts and multiple abstracts will be discussed.

\section{Case Two}

Intensivists and advanced practice providers (APPs) working on the east coast demonstrated that using Tele-ICU to supplement care has been associated with shorter length of stay (LOS) and lower mortality rates (Lilly et al., 2011). Using a 24/7 support model, the east coast Tele-ICU provides remote care planning and review of adult critically ill and injured patients, responds to alerts and alarms, and collaborates with local teams to focus on adherence to best practices. Results from a landmark study will be discussed.

A study has been conducted and results will be published in the near future on analyses of the financial impact of implementing the east coast Tele-ICU program. The primary outcome of this before and after study of an ICU telemedicine intervention was a change in contribution margin. Secondary outcomes included changes in case volume, net revenue, direct costs, case mix index, hospital length of stay, and the time to recover the initial capital investment. The intervention, which consisted of a Tele-ICU support model using intensivists and APPs that included use of the Tele-ICU intensivist as the gatekeeper to ICU beds at 7 ICUs in large academic center, will be discussed.

1. ATA TeleICU Practice Guidelines Work Group. (2014). Guidelines for TeleICU operations. ATA Standards \& Guidelines. Retrieved from http://www.americantelemed.org/resources/standards/ata-standardsguidelines

2. Goran S. A second set of eyes: An introduction to Tele-ICU. Crit Care Nurse 2010; 30(4):46-55.

3. Lilly C, Cody S, Zhao H, Landry K, Baker S, Mcllwaine J. Irwin R. Hospital mortality, length of stay, and preventable complications among critically ill patients before and after tele-ICU reengineering of critical care processes. JAMA 2011; 305(21):E1-9.

4. Lilly C, Thomas E. Tele-ICU: Experience to date. J Intensive Care Med 2010; 25(1):16-22.

5. Rincon T. Integration of Evidence-Based Knowledge Management in Microsystems: A Tele-ICU Experience. Crit Care Nurs Q 2012; 35(4): $335-40$.

6. Rincon T, Bourke G, Seiver A. Standardizing sepsis screening and management via a Tele-ICU program improves patient care. Telemed $J$ E Health 2011; 17(7):560-64.

\section{Objectives}

1. Discuss the experience (lessons learned) of Tele-ICU nurses in severe sepsis surveillance

2. Describe the strategies for achieving and sustaining local acceptance of Tele-ICU services

3. Outline the use of Tele-ICU services that can create an equitable and ethical ICU admission process

\section{P29 SHIFT HANDOFF COMMUNICATION AMONG NURSE PRACTITIONERS IN ACUTE CARE/TELE-ICU}

\section{PRESENTER:}

Katheryne T. Amba, DNP/PhDc, MSN, ACNP-BC, CCRN, Acute Care Nurse Practitioner

University of Chicago Hospital Goldfarb School of Nursing at Barnes Jewish College, St. Louis, MO

Introduction: More than a decade after the Institute of Medicine published the report; To err is human: Building a safer health system, (2000) concerns on patient safety have amplified as the public has become more responsive to the fatal consequences of medical errors. Shift handoff communication plays a critical role in addressing patient safety concerns in the healthcare system because miscommunication can lead to fatal medical errors. This session evaluates recent evidence on shift handoff among nurse practitioners in an attempt to establish a standardized format for shift handoff among nurse practitioners in an acute care setting or tele-ICU environment.

Methods: A literature search was performed on CINAHL, PubMed and The Cochrane Review over the last 15 years on studies and publications related to shift handoff in a hospital setting. Exclusion criteria eliminated studies that were not multidisciplinary, focused on bedside nursing reports and involved transitions of care out of the hospital setting. Inclusion criteria involved multidisciplinary studies on shift handoff in acute care environments/tele-ICU.

Results: There is a paucity of studies exploring shift handoff standards among nurse practitioners acute care environments/tele-ICU.

Conclusion: Patient safety is an important quality indicator in the US healthcare system. Miscommunication is a known major cause of sentinel events and improving communication process during shift handoffs may play an important role in decreasing medical errors. Despite the publications on medical errors and recommendations from organizations such as Joint Commission and The Institute of Medicine, the errors still persist and it is important for care providers to seek innovative methods to improve care processes that will decrease errors and enhance patient safety. Shift handoffs are an area that would benefit from the use of evidence-based practices to improve quality of care. Standardization of shift handoff protocols may be a start off point to improve shift handoff communication. It is important to consider the validity of tools currently in place to standardize communication so implementation can be beneficial to a multidisciplinary team of care providers. More research still needs to be conducted to evaluate the shift handoff process best practices. Since there has not been a randomized control trial to establish a bench mark on shift handoff process, more quantitative studies are required to demonstrate empirical data as to what form of best practice would support the shift handoff process.

1. Apker J, Mallak LA, Applegate EB, Gibson SC, Ham JJ, Johnson NA, Street RL. (2010). Exploring emergency physician-hospitalist handoff interactions: Development of the handoff communication assessment. Ann Emerg Med 2010; 55(2):161-70.

2. Arora VM, Johnson JK, Meltzer DO, Humphrey HJ. A theoretical framework and competency-based approach to improving handoffs. Qual Saf Health Care 2008; 17(1):11-14.

3. Benham-Hutchins MM, Effken JA. Multi-professional patterns and methods of communication during patient handoffs. Int J Med Inform 2009; 79(4):252-67.

4. Berger JT, Sten MB, Stockwell DC. The patient handoff: Delivering content efficiently and effectively is not enough. Int J Risk Saf Med 2012; 24(2), 201-5.

5. Kohn LT, Corrigan J, Donaldson MS. (2000). To err is human: Building a safer health system. Washington, DC: National Academy Press.

\section{Objectives}

1. Demonstrate awareness of the importance of standardized shift handoff communication during patient handoffs

2. Apply communication and change strategies to establish standardized shift handoff tool

3. Identify factors involved with implementing change in a tele-ICU/ Acute care practice

P30 TECHNICAL FEASIBILITY AND LIMITATIONS OF USING MOBILE TELEMEDICINE TO MEASURE THE NIH STROKE SCALE

\section{PRESENTER}

Tarin Casadonte, Master of Science - Industrial Engineering,

Health Systems Engineer

Mayo Clinic, Jacksonville, FL 
Introduction: Ischemic stroke is a time-based disease, with better outcomes linked to shorter times from onset to treatment. Reduction of door-to-needle time is associated with improved outcomes in IV/ rtPA eligible ischemic stroke patients. We hypothesized that remote ambulance-based measurement of the NIH stroke scale (NIHSS) using a HIPAA-compliant mobile platform immediately prior to arrival was feasible and would reduce door-to-needle times in patients eligible for treatment with IV rtPA.

Methods: We conducted a 3-part administrative, proof-of-concept feasibility pilot as part of our Comprehensive Stroke Center and stroke systems of care model (SSCM). The first part consisted of 'beta testing' a stroke patient scenario in simulation with EMS, over aspects of the NIHSS measurement using a HIPAA-compliant video platform. The second part was a 30-day feasibility period in the mobile EMS environment with timed measurement of NIHSS on stroke patients' en-route to our facility. All patients were treated by standard of care on arrival by the receiving neurologist/neurosurgery teams. Five EMS ambulances were equipped with a 4G/LTE enabled tablet preloaded with a secure HIPAA-compliant video application. The third part was a 30-day extension of the feasibility period to improve the logistical efficiency with EMS providers. The primary aim was video feasibility with timed measurement of the NIHSS. Secondary outcomes included satisfaction with the platform, comparing NIHSS measurement time against historical controls at our Stroke Center to calculate a theoretical 'DTN' time or impact.

Results: Part one of the pilot was successful in beta video testing a simulated patient scenario in 3 of 3 attempts. Part two was successful in video testing the mobile NIHSS measurement in 9 of 9 patients and part 3 was successful in 1 of 2 attempts. The one video attempt that was unsuccessful was due to local LTE being turned off on the device, which was later fixed. A separate attempt had the video signal drop twice during a severe thunderstorm, delaying, but not preventing the NIHSS measurement. NIHSS measurement time averaged $7.65 \mathrm{~min}$ in 10 of 11 total successful cases (range 3 to 9.8min). Satisfaction was rated good to excellent from the neurologist perspective, and satisfied to very satisfied in 100\% of successful attempts with EMS, and poor with the one failed video attempt. Given the average NIHSS measurement time, a theoretical DTN was $38 \mathrm{~min}$ with a theoretical impact, $\mathrm{P}$ - value 0.9 via paired t test. A power calculation using these data and standard deviation in DTN of $40 \mathrm{~min}$ showed that this theoretical DTN would require a sample size of 197 patients to test this hypothesis with an alpha of 0.05 with $80 \%$ power.

Conclusion: This proof of concept pilot demonstrates that remote ambulance-based $\mathrm{NIH}$ stroke scale measurement is feasible. The average time of the NIHSS measurement during transport was 7.65 minutes. Despite some technical limitations and the small sample size to adequately prove this hypothesis, this care model could have a positive impact on stroke patients as it theoretically reduced door to needle times by shifting clinical data collection to the pre-hospital setting and allowing patients to go directly to CT scan and potentially receive IV/ rtPA and/or interventions earlier.

1. Padrick M, et al. NIH stroke scale assessment via tablet-based mobile telestroke during ambulance transport is feasible - pilot data from the improving treatment with rapid evaluation of acute stroke via mobile telemedicine (iTreat) study. http://www.neurology.org/content/84/14_ Supplement/I1-2B

2. Wu TC, et al. Prehospital utility of rapid stroke evaluation using inambulance telemedicine: A pilot feasibility study. Stroke 2014; 45(8): 2342-47.

3. Kleindorfer D, et al. US geographic distribution of rt-pa utilization by hospital for acute ischemic stroke. Stroke 2009; 40(11):3580-584.

4. Schwamm LH, et al. A review of the evidence for the use of telemedicine within stroke systems of care: A scientific statement from the American Heart Association/American Stroke Association. Stroke 2009; 40(7):2616-634.

Objectives

1. Leverage HIPAA compliant mobile technology to evaluate patients in a moving ambulance
2. Expedite care of stroke patients through earlier Neurologist evaluation, while remaining Joint Commission compliant

3. Demonstrate potential reduction in average door to needle times of stroke patients

\section{Track: Chronic Care}

\section{SUNDAY, MAY 15, 2016}

5:35 PM-6:00 PM

Sunday, May 15, 2016

\section{EPOSTERS: MEET THE AUTHOR IN THE ATA EXPERIENCE ZONE/EXHIBIT HALL}

\section{P16 MAXOUT NETWORKS TO PREDICT HOME TELEMONITORING PATIENTS' ADVERSE EVENTS \\ PRESENTER:}

Kevin Mistry, Account Manager

AlayaCare, Montreal, QC, Canada

Introduction: As populations age, the number of patients with chronic conditions grows. This patient cohort is subject to frequent and costly hospital readmissions and emergency room visits. Those adverse events can often be prevented by a care worker intervention if foreseen. Home telemonitoring enables care workers to periodically assess their patients' health state by analyzing their vital signs. As shown in (Suh et al.), they mostly rely on manually setup ranges of acceptable values for every monitored data point. Complex rules that include multiple vitals can sometimes be used but are difficult to manage. Furthermore, making the rules evolve with the patient medical conditions requires human intervention. Machine learning is a potential solution. Even though it has been a game-changer in a number of industries from retail to finance, it has not yet been as widely adopted as it should in healthcare because it is seen by care workers as a black-box (Zhu et al.), i.e., hard to interpret. By using data gathered by a home healthcare agency who ran a 5 year home telemonitoring pilot with 320 patients, this research aims at providing experimental results to support the use of machine learning algorithms to predict home telemonitoring patients' adverse events.

Methods: Patients in our study were aged from 31 to 101 with an average age of 79 . Women represented $56.25 \%$ of the patient group. $26.56 \%$ of patients experienced at least one hospital readmission or emergency room visit while on the home telemonitoring program. The average number of events per patient was 0.49 with a standard deviation of 1.09 . 91.56\% of patients had comorbidities, hypertension being the more frequent at $55.31 \%$ followed by Chronic Heath Failure with 46.25\%, Diabetes with 39.69\% and chronic obstructive pulmonary disease at $38.13 \%$. In total, we had access to 76,359 observations representing a day of a patient's monitoring. For every observation, the manually setup alerts were available. Alerts were divided in three classes based on the care worker's configuration: low, medium, and high. The medical record registered diseases were first used to model a risk score of patients experiencing adverse events while on the program. This was done by modelling mean time between events through the use of a Cox Proportional Hazard (CoxPH) model. The monitored vital signs were used as covariates (X) alongside the patients' medical record registered diseases, vital signs' 1 and 2 days' log ratios, and risk score predicted for the patient by the CoxPH model. The indicator target variable $(\mathrm{Y})$ was coded to be true when the monitoring day was 1 of the 3 days preceding the day a patient experienced an event, so that care workers would be able to intervene. In the case of missing data, values were imputed with the mean value of the patient, and if unavailable, by the 


\section{ePOSTER PRESENTATIONS ABSTRACTS}

mean of all patient values for this vital sign. Then, the covariates were preprocessed with principal component analysis to lower the number of variables while maintaining more than $95 \%$ of the available variance. A state-of-the-art maxout neural network (Goodfellow et al.) trained with dropout was used to assess machine learning performance. Tenfold crossvalidation was used to make predictions on out-of-sample data. The models were trained with back-propagation and stochastic gradient descent. The optimal cutoff point to predict an event was optimized using a validation fold that represented 30\% of the training folds' number of observations. Because the adverse event class only occurred in $0.6 \%$ of all the dataset observations, we oversampled it on the training and validation folds, while not modifying the testing fold proportions. This used technique is often preferred to subsampling because it leverages all available data. Expanding on the evaluation of machine learning performance, we compared it to manually setup alerts. If an alert was triggered for an observation, we assumed that the care workers considered the day as an event for the patient. Likewise, if the predicted value for the observation was equal or above the cutoff point, we considered the observation as an alert.

Results: Out of the 447 events in the dataset, machine learning predicted 376 , while low alerts predicted 240, medium alerts 126, high alerts 109 and all alerts combined 340. Machine learning predictions resulted in 25,006 false positives while low alerts resulted in 40,080 , medium alerts in 18,847 , high alerts in 12,197 and all alerts combined in 54,384.

Conclusions: Our research shows that state-of-the-art maxout networks are very well suited to the task of predicting adverse events for home telemonitoring patients. Not only were they able to predict more events than manually setup alerts, but they also made half of the false positive errors that result in unnecessary work and over-diagnosis stress for the patients.

1. Goodfellow Ian J, David Warde-Farley, Mehdi Mirza, Aaron Courville, and Yoshua Bengio. "Maxout Networks." arXiv preprint arXiv:1302.4389 (2013). Google Scholar. Web.

2. Suh, Myung-kyung, Lorraine S Evangelista, Chien-An Chen, Kyungsik Han, Jinha Kang, Michael Kai Tu, Victor Chen, Ani Nahapetian, and Majid Sarrafzadeh. Proceedings of the 1st ACM International Health Informatics Symposium. N.p.: n.p., 2010. Google Scholar. Web.

3. Zhu, Mu, Lu Cheng, Joshua J Armstrong, Jeff W Poss, John P Hirdes, and Paul Stolee. Machine Learning in Healthcare Informatics. N.p.: Springer, 2014. Google Scholar. Web.

\section{Objectives}

1. Understand and assess how maxout neural networks can improve adverse events prediction

2. Understand how to evaluate machine learning models with out-ofsample observations and cross-validation

3. Understand how to use prediction models in a home healthcare agency

\section{P17 COYOT1 CLINIC: USE OF WEB-BASED VIDEO CONFERENCING} TO INCREASE ACCESS TO MEDICAL CARE FOR YOUNG ADULTS WITH TYPE 1 DIABETES

\section{PRESENTER:}

Jennifer Raymond, MD, MCR, Assistant Professor, Pediatric Endocrinology Barbara Davis Center for Diabetes, University of Colorado Anschutz Medical Campus, Children's Hospital Colorado, Aurora, CO

Backround: In patients with type 1 diabetes (T1D), glycemic control is at its worst during the late teens and early twenties and recent data from the T1D Exchange shows that glycemic control continues to worsen for this age group (Miller et al, 2015). Complicating matters, these young adults who are transitioning from childhood to adulthood, often lose connection with their diabetes care team (Duke et al, 2013 \& Edwards et al, 2014). The American Diabetes Association (ADA) recommends that patients with T1D complete a routine diabetes medical visit with an endocrinologist and a multidisciplinary diabetes care team four times per year, which doesn't successfully happen in the young adult population. The purpose of this study is to investigate the use of web-based video conferencing to increase access to evidence-based care and to reduce the barriers to care faced by young adults - to in effect, bring the care to them without interrupting their lives. Young adults, especially college students, are tech-savvy, but no research has been done using technology to engage young adults with T1D in routine diabetes care.

Methods: CoYoT1 (Colorado Young Adults with Type 1 Diabetes, said: coyote) Clinic was designed to meet the medical care needs of young adults (18-25 years) with T1D in a patient-based, shared medical appointment, teleenabled care model. Participants of CoYoT1 complete their clinic visits from home, work or other locations of their choosing, using HIPPA-approved, webbased, video conferencing. Through this method, patients are able to see their medical providers and interact with other young adults to gain psychosocial support. The requirements for participation include being an existing patient with access to the Internet via a device (e.g. I-Pad, laptop, PC, smart phone) equipped with a webcam and speakers. Participants complete their "virtual" clinic through a link they receive by email from their provider. Prior to the scheduled clinic visit, participants upload their diabetes devices and complete hemoglobin A1c $(\mathrm{HbA1C})$ measurements, and any other needed labs, at an outside laboratory (e.g. PCPs office, student health center or private lab company). Patients upload their devices to web-based programs; allowing the diabetes provider to access the patients' device reports via the Internet. The lab results are faxed from the testing laboratory to the provider at the diabetes center.

Results: Between April and August 2015, 45 patients completed a virtual CoYoT1 Clinic visit (mean age 19.8 years, mean HbA1C 8.7\%, 56\% female, 88\% non-Hispanic white). Prior to completing the virtual clinic visits, patients completed an average of 2.5 diabetes clinic visits in the preceding year. Only 10 patients $(22 \%)$ completed the recommended 4 visits in the preceding year and 10 patients (22\%) completed just one or fewer visits in the previous year; with 2 patients (4\%) having not been seen at all. At the time of enrollment in CoYoT1 Clinic, 13 patients (29\%) had not been seen in clinic in at least 5 months, but once recruited to CoYoT1 Clinic, they were seen within a few weeks. Prior to participation in CoYoT1 Clinic, patients reported losing an average of 0.67 days of work or school for a traditional, in-person, diabetes clinic visit. Comparatively, when patients completed their clinic visit virtually, via CoYoT1 Clinic, patients reported losing an average of 0.03 days from work or school; with only $2 \%$ of patients reporting any lost time. This resulted in a time savings of 0.64 days for patients completing their diabetes clinic visit via CoYoT1 Clinic compared to the traditional, in-person diabetes clinic visit $(p=<0.0001)$. Satisfaction surveys, completed by patients, found 95\% felt comfortable completing their medical visits via technology, 76\% thought the virtual clinic visit was easier to complete than an in-person clinic visit, 92\% would recommend the technology to others, and 95\% desired to have a virtual clinic visit again.

Conclusion: CoYoT1 Clinic may be an effective care model to engage young adults with T1D in routine diabetes care. Patients reported high satisfaction with the virtual clinic setting as well as a desire to complete a virtual clinic appointment again, which may result in increased visit frequency over time. The convenience and ease of completing a clinic visit from home resulted in significant time savings for patients, which may make it easier for young adults to fit diabetes clinic visits into their already busy schedules. Further evaluation of the CoYoT1 Clinic intervention is needed to determine if these results increase adherence to ADA standards and translate to improved glycemic control in this challenging population.

1. Duke DC, Raymond JK, Shimomaeda L, Harris MA. Recommendations for transition from pediatric to adult diabetes care: patients' perspectives. Diabetes Manag 2013; 3(4):297-4.

2. Edwards D, Noyes J, Lowes L, Haf Spencer L, Gregory JW. An ongoing struggle: a mixed-method systematic review of interventions, barriers and facilitators to achieving optimal self-care by children and young 
people with type 1 diabetes in educational settings. BMC Pediatr 2014 Sep 12; 14:228.

3. Miller KM, Foster, NC, Beck RW, et al. The T1D Exchange Clinic Network: Current state of type 1 diabetes treatment in the U.S.: updated data from The T1D exchange clinic. Diabetes Care 2015; 38(6):971-78.

\section{Objectives}

1. Describe applications of Web-based video conferencing for medical care

2. Identify how home use of Web-based video conferencing may increase access to medical care

3. Analyze the strengths and limitations of home-based telemedicine, using web-based video conferencing, for medical care
2. Nutescu E. Anticoagulation management services: Entering a new era. Pharmacotherapy 2010; 30(4):327-29.

3. Singh LG, et al. Implementation and outcomes of a pharmacist-managed clinical video Telehealth anticoagulation clinic. Am J Health-Syst Pharm 2015; 72(1):70-73.

\section{Objectives}

1. Describe the use of video technology as a tool to achieve individual patient therapeutic outcomes

2. Compare video-based anticoagulation management services to standard clinic-based care

3. Identify innovative strategies to manage high-risk medications in the home
P18 InNOVATIVE ANTICOAGULATION MANAGEMENT SERVICE: USING VIDEO TECHNOLOGY AND HOME POINT-OF-CARE TESTING IN AN INTENSIVE AMBULATORY CARE TELEHEALTH PROGRAM

\section{PRESENTER:}

Stephanie L. Schneck, PharmD, BCPS, BCACP, CACP, Clinical Pharmacy Specialist

Banner Health, Mesa, AZ

Warfarin has been the mainstay drug used to manage stroke and thromboembolic disease for over 50 years, and over two million patients in the United States are treated with warfarin today[1]. Routine medical care surrounding a physician office visit, designated anticoagulation clinics, and patient-directed self-testing are the three standard models currently in place to manage anticoagulation therapy. It is estimated that there are over 3000 anticoagulation clinics in the United States, yet over one-half of patients on warfarin therapy are still managed by the routine medical care model [2]. The routine medical care model includes patients traveling to the laboratory for blood work or an additional physician office visit solely for anticoagulation management. Anticoagulation clinics have been shown to improve safety and quality of anticoagulation management, yet oftentimes still require an inperson office visit to maintain therapeutic drug levels. Barriers to current care models include: delayed INR results reporting, delayed patient contact to adjust therapy and schedule follow up, time constraints on both the patient and the provider, financial burden for monitoring, and transportation to and from an anticoagulation clinic or physician office.

Telehealth has been a strategy to increase quality and efficiencies in anticoagulation management, yet most patient outreach is done telephonically or via virtual visits with a provider in a clinic setting [3]. In these cases care can be fragmented, and still requires an office visit to obtain INR results and manage anticoagulation therapy.

Banner iCare ${ }^{\mathrm{TM}}$ is an intensive ambulatory care model aimed at improving health care quality of life and reducing health care costs by utilizing telehealth technology. The Banner iCare ${ }^{\mathrm{TM}}$ program is a trans-disciplinary practice model that includes physicians, pharmacists, social workers, nurses, and health coaches to support the chronically ill through vigilant vital sign monitoring and outreach to detect adverse trends before they become adverse outcomes. The foundation of Banner iCare ${ }^{\mathrm{TM}}$ technology includes home-based monitoring equipment (such as a scale, blood pressure cuff, pulse oximetry, glucometer) along with a personal health tablet to enable video visits with patients in their homes. The development of patient-driven point-of-care INR testing, coupled with an anticoagulation management service helps to improve the quality of care, decrease complications associated with anticoagulation therapy, increase patient satisfaction, and reduce care gaps associated with high risk medication therapy. The virtual anticoagulation clinic greatly increases access to high-quality care without the patients leaving their home.

1. Ansell JE, Hughes R. Evolving models of warfarin management: anticoagulation clinics, patient self-monitoring, and patient self-management. Am Heart J 1996; 132(5):1095-100.

\section{MONDAY, MAY 16, 2016}

10:10 AM-10:35 AM Monday, May 16, 2016

\section{EPOSTERS: MEET THE AUTHOR IN THE ATA EXPERIENCE ZONE/EXHIBIT HALL}

\section{P19 HEART FAILURE READMISSION REDUCTION PROGRAM UTILIZING TELEMEDICINE}

\section{PRESENTER:}

Meghan E. Emig, MPAS, PA-C, Clinical Director, Advanced Heart Failure Carolinas HealthCare System, Charlotte, NC

Heart Failure (HF) is a chronic illness that affects more than five million patients in the United States and the prevalence is expected to increase by 25\% by 2030. The increasing cost burden is adding urgency to the need for consistent care. Much of the cost of HF is attributed to hospital admissions and readmissions. Many readmissions have been linked to variation in care. Although this patient population has been known to challenge compliance, a more proactive approach to education, communication and monitoring can significantly reduce the failure rate as measured by 30 day readmissions. As a result of the economic challenges associated with HF, Carolinas HealthCare System (CHS) recognized the need to address readmissions. HF is among the most common diagnoses across all CHS hospitals. It was recognized that there was a significant variation in managing $\mathrm{HF}$ across the continuum from primary care through complex inpatient services. An oversight team designed a phased approach to improving the quality of care for HF patients.

A navigator role was developed for $\mathrm{HF}$ with a goal to direct the course of the patient care as they transform form acute to ambulatory care after a HF admission. The nurse navigator is the patient advocate and also serves as the liaison to ensure the patients and families received needed resources. The Heart Success Transition Clinic (HSTC) was developed using a transitional care model to support the cross-continuum care for the HF patient. The nurse navigator enrolls each patient in the program with the first appointment within 3 to 5 days of discharge. The patient and family meet weekly with a HF specialty trained interdisciplinary team including an advanced clinical practitioner (ACP), nurse, pharmacist, social worker and dietitian. The care provided includes specialized HF monitoring and assessments, patient education, pharmacy support, social work support and diet education. The HTC team ensures the patient's HF condition remains stable as it relates to fluid management and clinical indicators. Nurse navigators track program enrollment and documented reasons for patient refusal for the HSTC program. This patient population was identified as "non-capture" and the majority of these patients was due to distance and transportation. As a result, CHS developed a virtual model of HSTC, partnering with a regional CHS facility. Patients with a $\mathrm{HF}$ admission at either facility could be enrolled in the virtual program. These 
patients connected with the HSTC team at the quaternary center via telemedicine, using basic videoconferencing technology. The value creation from the virtual program was evidenced by the number of miles and hours saved by the patient while receiving the same standard of care as patients enrolled in the HSTC at the quaternary center. There was also an increased compliance to follow up post discharge. Readmission rates in all three HSTC programs has shown dramatic declines in readmission rate and in the observed/expected ratio.

The HF team also utilizes device diagnostics to manage the need for HF admission or readmission. The early recognition and prompt treatment of decompensated HF has reduced the need for inpatient admission. Symptoms of HF are an insensitive marker of clinical decompensation and often represent an irreversible point in the congestion. Device-based diagnostics have been shown to predict clinical decompensation. Assessment of volume status by measuring thoracic impedance is effective in clinical practice. Utilization of device diagnostics accessed remotely allows convenient, regular assessment of HF patients at home. Through routine scheduling of transmissions, potential decompensation can be recognized early and therapy adjusted prior to symptoms. Remote monitoring has proven to be an excellent management tool and has assisted HF patients to better self-manage.

1. Grif Alspach J. Slowing the revolving door of hospitalization for acute heart failure. Crit Care Nurse 2014; 34(1):8-12.

2. Jones K, Kaewluang N, Lekhak N. Group visits for chronic illness management: implementation challenges and recommendations. Nurs Econ 2014; 32(3):118-47.

3. Kornburger C, Gibson C, Sadowski S, et al. Using "teach-back" to promote a safe transition from hospital to home: an evidence-based approach to improving the discharge process. J Pediatr Nurs 2013; 28(3):282-91.

\section{Objectives}

1. Demonstrate the use of technology to connect and provide care to patients in rural communities

2. Compare the traditional and virtual transitional care models

3. Summarize the effectiveness of using an interdisciplinary team approach to patients with chronic conditions

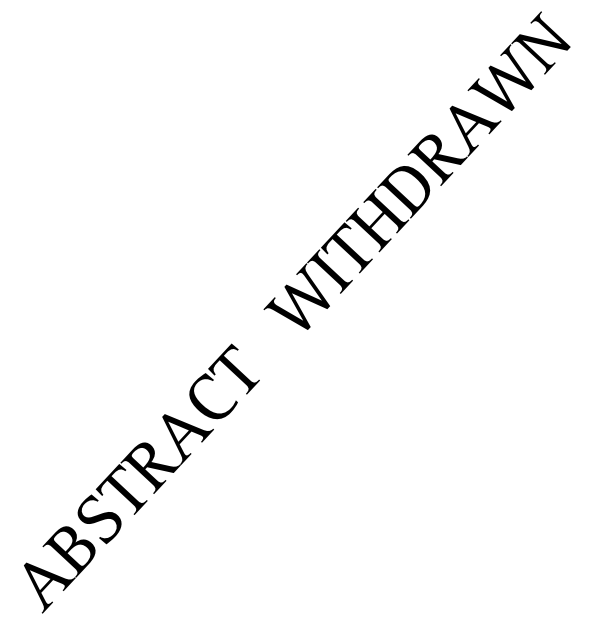

P21 AXON E-CLINICS: INNOVATIVE STRATEGIES TO DELIVER HEALTHCARE SERVICES FOR CHRONIC PATIENTS IN COLOMBIA

\section{PRESENTER:}

Carlos Augusto López, MD, MSc, Director

Glya Networks, Bogotá D.C., Colombia

The health system in Colombia, considering its nature of insurance, its goal of universal coverage and its limited resources available to support delivery models, needs innovative health services that transcend beyond conventional 


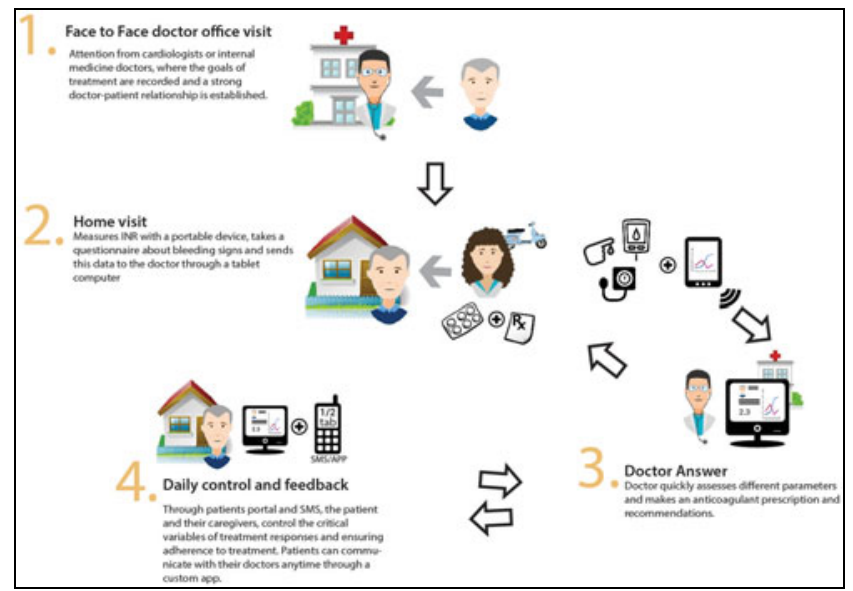

practices to ensure efficiency and expenditure rationalization, besides improving the timeliness and accessibility of the delivery.

The goal of the Axon E-Clinics is to develop innovative services that enable healthcare delivery to chronic patients, improving their quality of life, decreasing trips, improving doctor-patient relationship and creating a smart healthcare environment through information technology maximizing efficiency and service quality.

The Axon E-Clinics are a group of healthcare and technology strategies to treat patients with specific chronic diseases, using a two-stage scheme:

- One-time doctor office visit: using internal medicine doctors, doing a full EHR and establishing strong doctor-patient relationships and longterm treatments goals.

- Home visits and hometelecare: using medical assistants MA, IT driven care protocols and real-time doctor's surveillance.

- Clinics are developed specifically to treat disease groups with the following characteristics

- Diseases or conditions over 12 months of treatment

- Greater evidence available and full care protocols.

- Diseases with proper control and monitoring which reduce unnecessary medical events or complications.

- These clinics can bill for services packages, including drugs, labs and consultations.

Methods: Our first experience, Axon Anticoagulation E-Clinic manages patients on anticoagulant therapy, through monitoring of INR and other parameters, visit of a MA and remote monitoring by a cardiologist or internal medicine doctor. Patients of E-Clinic get face to face attention from cardiologists or internal medicine doctors, where the goals of treatment are recorded and a strong doctor-patient relationship is established. Thereafter, patients are monitored from home, where an MA visits them and measures INR with a portable device, takes a questionnaire about bleeding signs and sends this data to the doctor through a tablet computer who quickly assesses different parameters, including bleeding and stroke risk scores and makes an anticoagulant prescription, recommendations and sets the time of a new MA visit. Then the MA dispenses the anticoagulant, repeats doctor's recommendation, and hands a doctor's signed document with infographics details. The patient is sent an SMS reminder every day with the anticoagulant dose of the day. Patients at any time can start a conversation threat with their doctors through a custom app on their mobile phones.

Results: In six months of operation with 309 patients keeping a Time in Therapeutic Range - the TTR has been above 75\% while traditional methods do not exceed 55\%. When there has been two minor bleeding events, there has been an index of satisfaction $88 \%$ with the service. Additionally, city trips have dramatically decreased and maximized the use of scarce specialized doctors.

Conclusion: The Anticoagulation E-Clinic as a first experience of the Axon E-Clinics, improved the healthcare delivery output and increase of the profitability inside of a developing country insured healthcare model.

1. Pengo V, Pegoraro C, Cucchini U, Iliceto S. Worldwide management of oral anticoagulant therapy: the ISAM study. J Thromb Thrombolysis 2006; 21(1):73-77.

2. Taboada LB, Silva LE, Montenegro AC. Beneficios de la clínica de anticoagulación. Acta Med Colomb 2013 38:239-43.

3. Ocampo C, Hernández 0 , Velásquez C, Tobón I, Mejía F. La clínica de anticoagulación del Hospital Universitario San Vicente de Paúl: demografía, efectividad y complicaciones. Iatreia 2004; 17(2):105-14.

\section{Objectives}

1. Demonstrate an innovative method for treating chronic patients through mixed models of care

2. Identify within a telemedicine experience the importance of the innovative design of health model over the technology experience.

3. Develop smart healthcare models for developing countries

\section{P23 COMMUNITY PARAMEDICS' USE OF REMOTE MONITORING TO IMPROVE PATIENT CARE \\ PRESENTER: \\ Rick Whittaker, General Manager \\ Wellington Waterloo Futures Development Corporation, Elora, ON, Canada}

Remote Patient Monitoring (RPM) has been available for many years; however it has failed to gain a large uptake because it has not been well integrated into the existing care delivery system and because the technology costs have traditionally been quite high. The intent of this project is to integrate RPM into existing front line care providers, and demonstrate a business case for broader replication and sustainability of Remote Patient Monitoring.

The Community Paramedicine Remote Patient Monitoring (CPRPM) program is a two year, $\$ 2.1$ million, program that will provide RPM service to 1,500 patients, funded by Canada Health Infoway. As part of the program, Community Paramedics install Ideal Life Remote Patient Monitoring devices such as, Bluetooth enabled Blood Pressure Cuffs, Glucometers, Weight Scales and Pulse 0ximeters that automatically send the readings to the paramedics. Community Paramedics monitor patients with Congestive Heart Failure (CHF) and Chronic Obstructive Pulmonary Disease (COPD) and if the patient's readings fall outside of the thresholds established by the Clinical Advisory Committee or the patient's physician, an alert is triggered and the paramedic can follow up with the patient. Paramedics can be proactive to address the patient's current health status and can refer patients to other allied health care professionals such as the patient's physician and/or pharmacist when applicable. Constant patient monitoring allows patients to feel safe, and stay in the comfort of their homes longer.

To be eligible for this program, the patient must have a diagnosis of CHF or COPD and one of the following; three 911 calls, two emergency room visits, one hospital admission in the past 12 months. The effectiveness of the program will be evaluated by Queens University and success will be measured by a reduction in the number of 911 calls, reduced number of emergency room visits and admissions, and user satisfaction measurement of the program. The CPRPM program is currently being implemented in eight different counties across Ontario. There are over 170 patients currently enrolled in the program and early indications suggest a high level of satisfaction of both the RPM equipment and the Community Paramedic support.

The CPRPM program also connects the patient's family and health care professionals using an easy to use online web-based platform allowing the paramedics the ability to provide information to individuals in the patient's 
Circle of Care. On this platform, community paramedics are able to provide health care professionals biometric data reports generated by the patient's daily measurements. This up-to-date information can be used to assist healthcare professionals in making informed decisions on their patient's health care plans. This could include medication changes or dosage adjustments through the physician or pharmacist. Overall, the Circle of Care creates a communication dialogue between the family and circle of care members allowing for a more comprehensive and complete interdisciplinary approach to improving the patient's health.

In summary, the goal of this innovative program is to improve quality of life, help prevent 911 calls, hospitalization visits and admissions, as well as to improve communication amongst health care providers to deliver the highest quality of coordinated care for the patients. This is a unique RPM program as installation and monitoring is conducted by community paramedics. This presentation will discuss the unique lessons learned in the development, implementation, and launch of the CPRPM program.

\section{Objectives}

1. Understand how remote patient monitoring is being utilized in a universal healthcare system

2. Learn about alternative methods for deploying equipment and monitoring patients

3. Hear how this grant funded program is designed for scalability and continuation after funding ends

\section{P24 RETROSPECTIVE EVALUATION OF THE PHILIPS LIFELINE CARESAGE PREDICTIVE MODELS ON PATIENTS OF PARTNERS HEALTHCARE AT HOME}

\section{PRESENTER:}

Stephen Agboola, MD, MPH, Associate Director Connected Health Data Science \& Analytics

Partners Connected Health, Boston, MA

Introduction: The most common cause of emergency transports in elderly people (older than 65 years) is deterioration in their health status due to multiple chronic conditions that cause medical emergencies. The Philips Lifeline's CareSage program for elderly and frail people utilizes a Personal Emergency Response Service (PERS) with a unique AutoAlert feature that is designed for timely detection of medical emergencies and to promote independent living in the elderly. The PERS consists of a call button worn on the wrist or as a pendant that activates an intercom system in the home when pressed and directly connects the user with a response agent at a PERS callcenter. The system tracks the types and outcomes of all incidents. The intervention can potentially improve outcomes of emergency transport-related events. For example, falls occur in one-third of the elderly population per year resulting in over 2.5 million ED visits, 700,000 hospitalizations and about $\$ 34$ billion in direct medical costs. Timely detection of these falls and other medical emergencies is critical to optimizing outcomes. The purpose of this retrospective cohort study is to evaluate the clinical and financial impact of the Philips Lifeline's CareSage program with embedded PERS on patients of Partners Healthcare at Home. PERS is widely utilized but never as a source of data to identify patients at risk of emergency transports or ED visits or hospitalizations that cause huge healthcare expenditure. This is the primary objective of this study that makes it unique.

Methods: This evaluation of the Philips Lifeline PERS is a retrospective database review of the medical records of patients receiving care at any of the Partners Healthcare network of hospitals, enrolled in the Partners Healthcare at Home (PHH) program over the past 3 years (2012-2014) and who also subscribed to the Philips PERS. Currently, there are about 4,000 PHH patients who have subscribed to the Philips PERS. The data for this evaluation will be extracted from the Partners Healthcare Research Patient Data Repository - a clinical data registry that gathers medical records from various Partners
Healthcare-affiliated hospitals and stores them in a central location. Clinical outcomes (ED visits and hospitalizations) and financial outcomes (healthcare expenditure) will be assessed by multivariate regression models. Additionally, with the electronic medical record (EMR) as gold standard, we will also validate the predictive ability of an existing Philips Lifeline PERS algorithm to predict 30-day emergency transports using various performance measures such as model accuracy, sensitivity, specificity, positive predictive value (PPV), negative predictive value (NPV), area under the receiver operating characteristic (ROC) curve and diagnostic odd ratios.

Results: Our previous data analysis shows that 25\% of elderly patients become progressively more expensive yearly due to deterioration in their medical conditions. Clinicians need to identify these high risk patients and propose interventions to prevent possible clinical deterioration. This study is focused on identifying these patients. Data analysis is in progress and study results, expected later this year, will be updated before the conference.

Conclusions: We expect that predictive models based on PERS and EMR data will be valuable in identifying patients at risk of emergency transports, ED visits, hospitalizations that account for high healthcare cost. Hospitals and other healthcare organizations can use the outcome of the predictive models to design relevant interventions targeting their high risk patients.

\section{Objectives}

1. Demonstrate impact of the Personal Emergency Response Service on clinical outcomes

2. Demonstrate economic impact of the Personal Emergency Response Service

3. Delineate risk factors associated with emergency transports

\section{Track: Clinical Services}

\section{MONDAY, MAY 16, 2016}

5:30 PM-5:55 PM Monday, May 16, 2016

\section{EPOSTERS: MEET THE AUTHOR IN THE ATA EXPERIENCE ZONE/EXHIBIT HALL}

P31 GeRMWATCH: HEALTH INFORMATION TECHNOLOGY SUPPORTING POPULATION-BASED CLINICAL PRACTICE FOR ACUTE RESPIRATORY AND GASTROINTESTINAL TRACT INFECTIONS IN UTAH

PRESENTER:

Per Gesteland, MD, MSc, Associate Professor of Pediatrics and Adjunct Assistant Professor of Biomedical Informatics

University of Utah Dept. of Pediatrics and Intermountain Healthcare, Salt Lake City, UT

Background: Clinicians, patients and potential patients can benefit from timely, easily accessible, and region-specific information about communicable infectious disease activity in their communities. Access to such information has the potential to improve decision making in the context of extremely common conditions that account for a majority of acute, ambulatory healthcare utilization and are associated with threats to patient safety (e.g., unnecessary antibiotics, adverse drug events) and patient satisfaction (e.g., complaints relating not getting an antibiotic).

Objective: Develop a suite of technologies aimed at providing information tailored to the needs and work flows of clinicians, patients and potential healthcare consumers. 


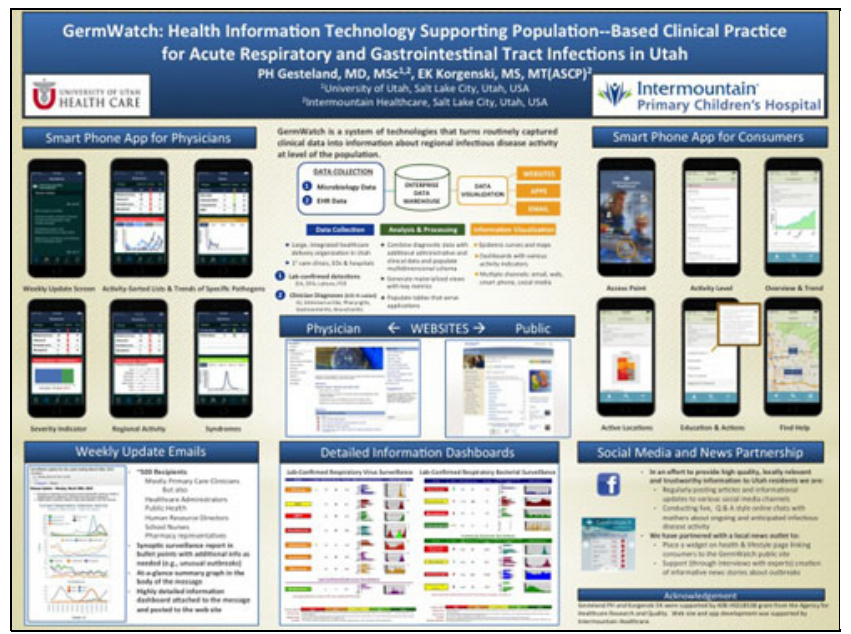

Design/Methods: We used 1) focus groups with clinicians and parents of patients, 2) key informant interviews and cognitive task analyses with practicing clinicians and 3) iterative design principles to inform the design and development of multiple approaches for disseminating information about regional infectious disease activity in Utah.

Results: Over 5+ years, we developed a collection of technologies including: an automated data collection and warehousing system; visual analytic and information displays; a provider-facing web site and iPhone application; an email distribution list comprised of $\sim 500$ individuals (clinicians, public health partners, school nurses, HR managers, healthcare facility administrators) who receive weekly updates; a public-facing web site and companion cross-platform mobile application; a partnership with local news media to disseminate the "viral weather report"; and a process for harnessing the power of social media to engage consumers in discussion/education about upcoming and ongoing outbreaks.

Conclusions: Both clinicians and the public are eager to have access to high quality information from a trusted source. GermWatch provides a compelling demonstration of how modern technologies can be combined to meet this information need.

\section{Objectives}

1. Understand modern approaches to infectious disease surveillance

2. Understand how health information technologies can be combined to support provider and consumer situational awareness about regional infectious disease activity

3. Understand how these technologies have the potential to impact the efficiency, quality and safety of health care delivery in the context of acute infectious diseases

\section{P32 SHOW-ME ECHO: A KNOWLEDGE-SHARING TELEHEALTH PARTNERSHIP FOR CO-MANAGEMENT OF CHRONIC PAIN}

\section{PRESENTER:}

Mirna Becevic, PhD, MHA, Assistant Research Professor

University of Missouri, Columbia, MO

Background: Primary care providers (PCPs) encounter a wide array of conditions in their clinics on any given day. In addition, rural providers experience professional isolation, and frequent inability of their patients requiring specialty care to travel to large medical centers for treatment. One common patient complaint in primary care is chronic pain ${ }^{1}$. Some studies report that as much as $1 / 3$ of weekly adult visits in a primary care setting are chronic painrelated $^{1}$. However, PCPs report dissatisfaction and concerns with their training in chronic pain, the availability of CMEs related to chronic pain issues, opioid abuse and addiction, and decreased patient compliance ${ }^{1}$.

Introduction: ECHO stands for Extension for Community Healthcare Outcomes. It is a knowledge-sharing network linking academic health care centers to rural providers via telehealth, facilitating co-management of chronic diseases ${ }^{2}$. Developed at the University of New Mexico by Dr. Sanjeev Arora to alleviate the burden of hepatitis $\mathrm{C}$ in rural New Mexico created by the inability of patients to seek timely and adequate care, resulting in sicker patients and higher mortality rates ${ }^{2}$. ECHO is a weekly or bi-weekly telehealth 'clinic' where rural providers connect to a multi-specialty team at an academic medical center and present de-identified cases in a case-based learning format. Multi-specialty teams mentor and educate PCPs on management and treatment of those patients. Each clinic includes a CME-approved didactic presentation related to the specific condition. ECHO has been replicated in over 40 disease states in the US, US Department of Defense, and abroad. The Missouri Telehealth Network (MTN) at the University of Missouri (MU) started a pilot Chronic Pain ECHO in October 2014, ending in June 2015. After this pilot, Chronic Pain ECHO continued, utilizing the knowledge and data collected during the pilot to improve and extend the services. Chronic Pain ECHO utilizes technological advances to facilitate the ECHO clinic. This technology, accomplished through the use of software called Zoom, utilizes cameras and speakers located on most current pcs, laptops, cell phones, tablets and other devices. Zoom is a cloud-based software that installs a client application on end-users' devices that enables them to connect to the Zoom server. Once connected, users are able to fully engage each other via questions and answers. Not only will Zoom accommodate most every device, it also works seamlessly with other technologies, including Polycom. The Chronic Pain ECHO multi-disciplinary hub team consists of a pain management physician (MD or D0), clinical psychologist (PsyD or PhD), pharmacist (PharmD), and an RN. The hub team operates out of the Missouri Primary Care Association (MPCA). The participating sites include Federally Qualified Health Care Centers (FQHCs), and rural health clinics in Missouri.

Materials and Methods: In order to learn about the participating providers' perceptions and satisfaction with the Chronic Pain ECHO, we conducted a post-satisfaction survey, consisting of 33 questions, at the end of the pilot. The study was approved by the MU Institutional Review Board, number 1213197. Data was collected online, via REDCap. Initially, two locations with an average of 14 PCPs attended the pilot ECHO. As the recruitment of providers continued, there were 4 locations with an average of 22 PCPs at the end of the pilot. A total of 6 PCPs participated in the study, with only one submitting the completed survey, and 5 leaving some of the questions as incomplete. This resulted in $27 \%$ participation rate.

Results and Conclusion: Seventy five percent of respondents to the survey indicated highest learning with treatment of behavioral health and substance abuse issues, 50\% in management of side effects associated with patients being treated for chronic pain. Fifty percent of the respondents indicated moderate learning in identification of patients eligible for chronic pain treatment. However, participating providers were divided in new knowledge in screening patients for chronic pain or pharmacological management, with half of the participants indicating no learning, and another half moderate or high learning. Clinic observations show high participant engagement during case and didactic presentations. In a largely rural state, such as Missouri, ECHO provides not only access to education and specialty consultations, but also builds peer relationships and support. Our obvious challenge was small sample size of providers. However, this was the very first ECHO clinic in Missouri and provider recruitment was expected to be a main challenge. After the pilot, 22 additional providers signed up to participate in Chronic Pain ECHO. It is expected that 20 sites will be participating in Chronic Pain ECHO by the end of year 1 of the project. This year the Missouri Senate appropriated $\$ 1.5 \mathrm{M}$ in the higher education appropriations bill, HB3 for Show-Me ECHO, supporting 6 ECHO Projects in 2015: chronic pain, autism, endocrinology, hepatitis C, dermatology, and pediatric asthma. Findings from this study, as 


\section{ePOSTER PRESENTATIONS ABSTRACTS}

well as clinic observations, are used as quality improvement tools to develop and manage all ECHO clinics started between 2014 and 2015.

1. Upshur CC, Luckmann RS, Savageau JA. Primary care provider concerns about management of chronic pain in community clinic populations. J Gen Intern Med 2006: 21(6):652-55.

2. Arora $\mathrm{S}$, et al. Academic health center management of chronic diseases through knowledge networks: Project ECHO." Acad Med 2007; 82(): 154-60.

\section{Objectives}

1. Recognize the implications and difference between 1:1 telemedicine vs. knowledge-sharing network

2. Identify successes and challenges with building a state-wide ECHO project

3. Generate ideas on possible other applications on ECHO projects

\section{P33 INCREASED IDENTIFICATION OF DIABETIC RETINOPATHY AND REDUCTION IN UNGRADABLE IMAGE RATE WITH ULTRAWIDE FIELD IMAGING WITHIN A NATIONAL TELEOPHTHALMOLOGY PROGRAM}

\section{PRESENTER:}

Paolo S. Silva, MD, Ophthalmologist

Harvard Medical School, Joslin Diabetes Center, Boston, MA

Introduction: To compare DR identification and ungradable image rates between nonmydriatic ultrawide field imaging (UWFI) and nonmydriatic multi-field fundus photography (NMFP) in a large multi-state populationbased diabetic retinopathy (DR) ocular telehealth program.

Methods: Within the Indian Health Service - Joslin Vision Network (IHSJVN) program which serves American Indian and Alaska Native communities at 97 sites across 25 states, all patients undergoing JVN imaging either with NMFP or UWFI from May 1, 2014 to August 30, 2015 were evaluated. All retinal images were graded using a standardized validated protocol in a centralized reading center.

Results: 35,052 eyes of 17,526 patients were imaged using NMFP and 16,218 eyes of 8,109 patients were imaged using UWFI. The ungradable rate per patient for DR and DME was significantly lower with UWFI compared to NMFP (DR: $2.8 \%$ vs $26.9 \%, p<0.0001$; DME: $3.8 \%$ vs $26.2 \%, p<0.0001$ ). Identification of eyes with either DR or referable DR (moderate nonproliferative DR or more severe levels of DR or presence of DME) was increased using UWFI from $11.7 \%$ to $24.2 \%(\mathrm{p}<0.0001)$ and $6.2 \%$ to $13.6 \%(\mathrm{p}<0.0001)$, respectively. In eyes with DR imaged with UWFI ( $N=3,926$ eyes of 2,402 patients), the presence of predominantly peripheral lesions suggested a more severe level of DR in $7.2 \%$ of eyes and 9.6\% of patients.

Conclusions: In a large, widely-distributed DR ocular telehealth program, nonmydriatic UWF imaging reduced the number of ungradable eyes nearly $90 \%$ (to <3\%), increased the identification of DR nearly 2 -fold and identified peripheral lesions suggesting more severe DR in $9.6 \%$ of patients.

1. Silva PS, Cavallerano JD, Tolls D, Omar A, Thakore K, Patel B, Sehizadeh M, Tolson AM, Sun JK, Aiello LM, Aiello LP. Potential efficiency benefits of nonmydriatic ultrawide field retinal imaging in an ocular telehealth diabetic retinopathy program. Diabetes Care 2014; 37(1):50-55.

2. Wilson C, Horton M, Cavallerano J, Aiello LM. Addition of primary care-based retinal imaging technology to an existing eye care professional referral program increased the rate of surveillance and treatment of diabetic retinopathy. Diabetes Care 2005; 28(2):318-22.

3. Silva PS, Cavallerano JD, Sun JK, Noble J, Aiello LM, Aiello LP: Nonmydriatic Ultrawide Field Retinal Imaging Compared with Dilated Standard 7-Field 35-mm Photography and Retinal Specialist Examination for Evaluation of Diabetic Retinopathy. Am J Ophthalmol 2012; 154(3):549-59.
4. Kernt M, Hadi I, Pinter F, Seidensticker F, Hirneiss C, Haritoglou C, Kampik A, Ulbig MW, Neubauer AS: Assessment of diabetic retinopathy using nonmydriatic ultra-widefield scanning laser ophthalmoscopy (Optomap) compared with ETDRS 7-field stereo photography. Diabetes Care 2012; 35(12):2459-63.

\section{Objectives}

1. To determine the efficiency benefits of ultrawide field retinal imaging in UWFI within a nationally distributed teleophthalmology program

2. To determine the distribution and proportion of diabetic retinal findings on ultrawide field imaging within a nationally distributed teleophthalmology program

3. To determine the proportion of patients with peripheral diabetic retinopathy lesions on ultrawide field retinal imaging that would not have been identified with routine retinal photography

\section{P34 USE OF TELEMEDICINE TECHNOLOGIES AND SYSTEMS TO INCREASE ACCESS TO HIV/AIDS SPECIALTY CARE AND TREATMENT IN ZAMBIA}

\section{PRESENTER:}

Kelley Kesler, MS, Director of Special Projects

Global Partnership for Telehealth, Waycross, GA

With a growing population of over fifteen million people, the African county of Zambia is facing a huge health crisis. Zambia currently is fighting an HIV/AIDS epidemic, but the larger challenge is the lack of physicians to care for their population. Currently, there is one physician for every twenty three thousand individuals. As a nation, Zambia realized the physician shortage would not allow proper access to medical care for their residents or allow the control of the HIV/AIDS epidemic.

In an exceptional partnership, Global Partnership for Telehealth (GPT) has joined with the American International Health Alliance (AIHA) and Zambian Department Force (ZDF) to develop and implement a telemedicine program for the Zambian military and Zambian citizens located in neighboring villages.

ZDF oversees more than 50 health sites across the country that serve both civilian and military populations, which puts it in an excellent position to take the lead on new and cutting-edge health initiatives like telemedicine. Because many of these health sites are situated in rural areas and lack access to specialized care, patients - particularly those living with HIV or other complex or chronic conditions - often face significant barriers to accessing health services, including time, distance, transportation, and cost concerns. Telemedicine effectively eliminates these barriers and therefore has great potential to reduce morbidity and mortality rates, as well as to improve the quality of life for people living in rural areas.

The project is designed to improve provider access to evidence-based clinical resources as a means of enhancing care management for PLHIV and other military and civilian patients treated at ZDF clinical sites throughout the country.

On September 3, 2015 in Lusaka, Zambia Working in partnership with the Government of Zambia, the Zambian Defense Forces (ZDF) Military Medical Services, and the American International Health Alliance (AIHA), Global Partnership for Telehealth celebrated the launch of a telemedicine initiative that will improve access to high quality diagnostics and specialized care to people living in rural, underserved parts of Zambia. Supported by the US President's Emergency Plan for AIDS Relief (PEPFAR) and the US Department of Defense in Zambia, this innovative national eHealth initiative links clinical experts at Maina Soko Military Hospital with pilot ZDF health sites in Lusaka West, Mansa, Kabwe, and Kaoma, as well as at the Defense Force School of Health Sciences in Lusaka.

As with any program implementation there were challenges to solve and overcome, but with innovative and collaborative partnerships there isn't a challenge that cannot be solved. This innovative partnership is the first of its 


\section{ePOSTER PRESENTATIONS ABSTRACTS}

kind for Zambia. Telemedicine is necessary for future Zambians to have access to quality medical care and to gain control over the HIV/AIDS epidemic.

AIHA Partners Launch New Telemedicine Initiative in Zambia. (2015, September 3). Retrieved September 3, 2015, from http://www.AIHA.com

\section{Objectives}

1. Describe the challenges to be addressed while planning for an international program

2. Define an effective partnership

3. Describe the benefits of an effective partnership

\section{P35 InPATIENT VIRTUAL ROUNDING \\ PRESENTER:}

Stephen D. Cassivi, MD, Consultant

Mayo Clinic, Rochester, $M N$

Background: A multidisciplinary team assembled to design and test workflow and technology processes to create a virtual rounding interaction in the inpatient setting. The team tested an experimental prototype and the feasibility and value of a virtual telehealth process that permits a surgeon/physician to interact with the patient via video, utilizing an electronic media tool.

Methods: Pros and cons were considered including the effectiveness of existing technologies: wall-mounted unit, telemedicine Cart (plug-in cable connection), and an iPad (wireless connection). Criteria included ease of use for surgeons/providers, intimacy and comfort for the patient, cost, as well as functionality, convenience and scheduling flexibility. The team aimed at demonstrating the ability to bring together a meaningful and efficient clinical conversation in a safe, convenient and secure mechanism. The iPad technology was chosen for the study. The team worked with the health unit coordinator (HUC) and call-routing software (SBR) to develop a process to make the connection seamless and fit within the work flow of the clinical practice. Five surgeons and one hospitalist participated in the six-month pilot (January 2015 - July 2015), with 23 unique patients and 34 patient - provider virtual visit connections. Patients and providers were surveyed to gather immediate feedback and ensure their needs were being met.

Results: This pilot demonstrated efficiency and satisfaction of virtual rounding. Patient satisfaction with the service earned 4.9 on a 5.0 Likert scale. All six providers reported 100\% satisfaction, stating use of the iPad met their needs as a convenient and efficient communication tool. Providers were able to utilize iPhone technology to connect to the patient via iPad from around the globe. Feedback gathered prompted the team to execute small enhancement cycles to improve the system efficiency, reliability and patient and surgeon experience using an iPad. The team was able to identify situations when virtual rounding had value and when face to face interaction was not required.

Conclusion: Inpatient virtual rounding offered a mobile innovative option for healthcare providers to interact with the patient. The iPad provided a cost effective electronic media tool when the patient did not require a hands-on face to face patient encounter or a face to face visit was impractical. The virtual rounding tool was developed as part of work flow of the inpatient unit and improved coordination and connectivity between primary providers, consulted services, interpreters and the patient - creating a secure and convenient patient experience.

1. Gardner MR et al. Perceptions of Video-Based Appointments from the Patient's Home: A Patient Survey. Telemed J E Health 2015; 21(4): 281-85.

\section{Objectives}

1. Recognize the feasibility and value of a virtual telehealth process that permits a surgeon/physician to interact with the patient via video

2. Understand the efficiency in practice through a convenient and simple technological mechanism

3. Identify the use of design principles in evaluating various technical platforms to determine the most effective systematic process

\section{P36 TELEREHABILITATION AND TREATMENT ALGORITHM OF VICTIMS WITH COMBAT INJURIES OF THE LOWER EXTREMITIES \\ PRESENTER:}

Andriy J. Hospodarskyy, PhD, Associate Professor

Ternopil Medical University, Ternopil, Ukraine

Background: Orthopedic rehabilitation is a subspecialty that involves the care of patients who have complex musculoskeletal problems. Rehabilitation combines physiology and biomechanics in a unique manner that focuses on improving the patient's functional outcome and overall well-being. After the injury in the body of the victim, physiological constants are temporarily violated, causing protective, restorative and compensatory reactions. With timely start of physical activity rehabilitation of injured muscle occurs due to functional loading (exercise, massage, training, dosed walking with or without crutches). Currently the sequential algorithm of dosed loads on the injured extremity after immobilization and postoperative treatment of victims is not sufficiently studied, nor is the physiological and pathophysiological response for the sequence and dose of axial load on the limb.

Aim: Develop a computerized system ("server and the doctor" - "patient and sensor") which allows performing remote monitoring and correction of dosed axial load on injured limbs in real time.

Methods: We monitored patients after early immobilization with injuries of the lower extremities. All patients underwent generally accepted methods of rehabilitation in hospital (massage, myostimulation, walking without a load on the injured limb, and exercises in the pool). Patients were trained with a set and sequence of exercises at home:

- Fixation of the smartphone to the injured limb and working with software

- Passive flexion-extension, abduction in the hand, a circular motion in adjacent joints

- Active flexion-extension, abduction in the hand, a circular motion in joints

- Dosed load on injured limb by using crutches

- Dosed load on injured limb without using crutches

Patients trained with the method of assessment of pain on a 10-point scale, the method of measuring the volume of soft tissue and compared it with healthy limb before and after each stage of the exercise.

Results: Three patients with combat injuries of the lower extremities affected more than 2 months prior participated in the telerehabilitation program. The system of remote monitoring includes smartphones (with gyroscope, G-sensor, magnetometer, barometer) fixed to the injured limb and software. During the execution of exercises data from the smartphone's sensors goes to a server through the internet and to the personal smartphone of the doctor and is displayed as digital data and graphs. During the telemonitoring the physician controls the adequacy of execution of each stage of rehabilitation exercises and has the ability to adjust the load in real time depending on the functional state of the limb. Software allows monitoring the exercises time, the frequency of active movements of the injured limb, the number of steps per hour, walking speed, and heart rate changes. The 3-month rehabilitation period started with the power load of $10 \%$ of body weight, following 3-5 steps five times per day with a speed no more than $1 \mathrm{~km} / \mathrm{h}$. The algorithm allows increasing the daily load 1\% if the assessment of pain after exercise was not more than 7 points and progressive limb edema was absent. Otherwise, the patient was invited to visit the doctor with the next correction of rehabilitation algorithm. We propose the use of telerehabilitation as a key component in the long-term management of combat-wounded veterans who have lower extremities injuries.

Conclusion: Telerehabilitation is a good alternative for providing access to specialty care for the growing number of service members returning with traumatic injuries. Modern smartphones, computer networking, and software 
systems allow for the rehabilitation and treatment of patients in various locations, including the home environment. Telerehabilitation systems and dosed load algorithms can be used in complex rehabilitation of victims with combat injuries of the lower extremities. This will improve the quality of life in this group of patients and significantly reduce the cost of the rehabilitation period.

1. Baldwin K, Namdari S, Hosalkar H, Spiegel DA, Keenan MA. What's New in Orthopaedic Rehabilitation. J Bone Joint Surg Am 2012; 94(22):2106-111.

2. Doarn CR, McVeigh F, Poropatich R. Innovated new technologies to identify and treat traumatic brain injuries: crossover technologies and approaches between military and civilian applications. Telemed $J E$ Health 2009; 16(3):373-81.

3. Hauret KG, Jones BH, Bullock SH, et al. Musculoskeletal injuries; description of an under-recognized injury problem among military personnel. Am J Prev Med 2010; 38(1S):S61-70.

\section{Objectives}

1. Use modern smartphones as health sensors

2. Study telerehabilitation procedure

3. Telemonitoring patients

\section{MONDAY, MAY 16, 2016}

5:55 PM-6:20 PM Monday, May 16, 2016

\section{EPOSTERS: MEET THE AUTHOR IN THE ATA EXPERIENCE ZONE/EXHIBIT HALL}

\section{P38 EXCLUSIVE BREASTFEEDING EDUCATION AND SUPPORT FOR COMMUNITY NURSES IN TRIFINIO, GUATEMALA \\ PRESENTER: \\ John F. Thomas, IV, PhD, MSSW, Director of Telehealth, Co-Director of ECHO Colorado \\ University of Colorado, Childrens Hospital Colorado, Aurora, CO}

Exclusive breastfeeding provides optimal infant nutrition and health benefit for both the mother and baby dyad. The Trifinio area is an impoverished region in the coastal lowlands of southwestern Guatemala with a population of approximately 25,000 inhabitants. Children there are at high risk for malnutrition, parasites and diarrhea that are often linked to the lack of potable water, unsanitary living conditions and food insecurity. As part of the community home visitation program with nurses and comadronas (midwives) of Trifinio, formalized teaching was implemented with on-site classes as well as remote weekly Vidyo sessions. Curriculum includes content from World Health Organization, Breastfeeding Telephone Triage and Advice Book (author MB) translated into Spanish and the Wellstart International Lactation Self-study Modules. Topics addressed are exclusivity (no need for water), 10 screening questions to assess for concerns, true insufficient milk supply, hand expression when dyad is separated, poor weight gain, sore nipples, engorgement, correct latch, unusual nipple and breast shape/size, mastitis, complementary foods at age 6 months and in older infants caution with grazing and more solids besides caldito (soup). Close follow-up when nursing is not going well is key to success because failure to address lactation issues has significant financial and nutritional implications in this population. Moreover we emphasize the importance of observation of breastfeeding sessions during home visits with clinic referrals if problems are noted. Teaching tools used include weekly video-enabled 45 minute sessions that include short Powerpoint presentations (3-5 slides), photos of common problems, case presentations and interactive discussion.

\section{Objectives}

1. Understand the benefits of exclusive breastfeeding

2. Understand the impact of poverty and education in rural Guatemala

3. Understand the barriers to health and well-being in rural Guatemala

\section{P39 TELECONSULTATION AS ORTHOPEDICS QUEUE APPROACH STRATEGY IN THE BRAZILIAN HEALTH SYSTEM}

\section{PRESENTER:}

Alaneir F. Santos, MD, PhD, Vice Chair Department of Social Medicine Federal University of Minas Gerais, Belo Horizonte, Brazil

Introduction: The Brazilian National Telehealth Program started in 2006 as a pilot project in nine states to support primary care in order to improve the quality of care in the Unified Health System (SUS in Portuguese). Currently, the program is implemented in all Brazilian states. The main activities are: teleconsultation, remote diagnostics and tele-education depending on the expertise of each nucleus. It is noted that these activities are still underutilized. To address this problem the telehealth team of the School of Medicine of the Federal University of Minas Gerais (FM/UFMG) and the Belo Horizonte Health Department set out to develop a methodology to stimulate the use of teleconsultation as a tool to reduce queues in specialized consultations in SUS. Access to consultations and specialized tests has been a problem in all regions of Brazil. The causes of this problem are manifold: A. Low resolubility of primary care, whose professionals are still in training to assist population; B. Market supply of medical specialists with specific characteristics in each specialty, making it difficult to hire these professionals, and as a consequence low supply of appointments and tests, generating large queues; C. Lack of updated parameters needed for specialized consultations creating difficulties in planning and regulation of demand and supply; D. Lack of an information system for assessing the path taken by the patient in the system leading to irrational use of diagnostic and therapeutic resources. A pilot project was proposed in the orthopedic specialty, one of the three specialties with the highest number of people in the queue. In this area, the cumulative queue of patients requiring specialty was 1,900 patients in November 2014 at 21 primary care units (UBS in Portuguese) in the Northeast Health District of the City of Belo Horizonte - Brazil.

Objective: The aim of the project was to develop a method in which the Teleconsultation be used in a planned and managed way, impacting on the qualification of referrals to specialists and reducing waiting list.

Methods: The project was developed from November 2014 to May 2015 in UBS that integrate the regional Northeast and accumulated a row of 1,918 patients waiting for a specialist consultation in Orthopedics, following the steps:

- Orthopedics protocol update with the inclusion of teleconsultation and referral flow

- Talk with district managers and managers of health facilities to define deployment strategies

- Training of doctors in health centers in orthopedics protocol and in the use of telehealth resources

- Calling patients of to clinical reassessment performed by community health workers, by phone or home visit

- Structuring a flow of attention that allowed the realization of the MRI scan suggested by teleconsultants

Results and Discussion: Nine UBS were evaluated which had a total of 777 patients queue. When the quantitative systematization of data was made, it was observed that participated in the project 619 patients: $271(43 \%)$ had administrative discharge; 239 (39\%) were reevaluated clinically and 109 patients (18\%) did not attend the revaluation. Of these 239 patients clinically investigated by the family health doctors, it was observed that 81 patients (33\%) were discharged from the clinic, 39 patients (17\%) were referred to the UBS support services and 119 patients (50\%) had their cases discussed via teleconsultation. Of the 119 cases evaluated through teleconsultation, a significant number of non-registration of conduct adopted by the UBS professional, so 94 patients $(78 \%)$. Of the recorded cases $19(15 \%)$ remained in the facility or were referred to support services and $9(1 \%)$ remained on the waiting list of orthopedics, but had the degree of priority reclassified. In addition to the direct benefit to patients, there was also the training of professionals to work in similar cases. This data points to the importance of 


\section{ePOSTER PRESENTATIONS ABSTRACTS}

training in the use of institutional orthopedics protocol, as well as the advantages of the use of teleconsultation tools. It also pointed out the difficulty of available time on the work agenda for this activity, due to high demand for medical attention.

Conclusion: The measures used to reduce the waiting list were very effective, reducing the queue by $47 \%$ when considering discharges after revaluation (352) and after performing the teleconsultation (19). One believes this methodology can be reproduced for other specialties. Another point to note was the incorporation, after the project, of telemedicine as a key tool in improving the resolubility of primary care.

\section{Objectives}

1. Compare and exchange experience

2. Demonstrate the use of telehealth resources to reduce specialist waiting queue

3. Analyze the possible reproducibility of the project

\section{P40 USING TELEHEALTH MODALITIES TO DELIVER PRACTICE-BASED WEIGHT MANAGEMENT INITIATIVES TO RURAL COMMUNITIES \\ PRESENTER: \\ Ragan Aleise DuBose-Morris, PhD, Director of Telehealth Education Medical University of South Carolina, Charleston, SC}

Compounding an established lack of healthcare access in rural communities, providers are challenged in the management of patients who are diagnosed as overweight or obese. Given the high prevalence of overweight and obesity, it is essential that rural primary care providers be prepared and willing to address weight management with their patients. However, when it comes to obesity treatment, the problem of limited access is exacerbated by the fact that many healthcare providers do not adequately address excess weight with their patients. Weight-related bias and discomfort, pessimism about obese patient compliance, feeling inadequately trained and unconfident to treat overweight and obesity, and lack of insurance reimbursement have been cited as major barriers to providing weight management treatment.

As for the types of treatment recommended to treat obesity, in 2012, the US Preventive Services Task Force (UPPSTF) officially recommended "intensive, multicomponent behavioral interventions" for obese adults. These types of specialty services are typically not available in rural communities. In addition, primary care providers often lack the necessary practice resources and technical infrastructure required to link with specialty services through telehealth applications.

Through a quality improvement project for practice support (Wellness Connect), practices have been brought together across South Carolina to serve overweight and obese patients in a mix of geographic and practice-type settings. Telehealth technology is not only providing rural clinics with access to an interprofessional team of weight management experts, it is also supporting patients through the use of m-Health applications designed solely for collaborative use between the providers and clinical faculty to help monitor patient progress.

The project consists of: online education for clinical personnel and students covering a number of obesity-related topics that reflect the broad and interprofessional nature of the disease; bi-weekly group patient sessions led by a psychologist, registered dietitian, and exercise physiologist using videoconferencing systems; and a provider-focused mobile app that captures weight and blood pressure data from wireless peripherals while allowing manual input of height, hip and waist circumference, and self-reported survey responses. The tools and processes are constructed in such a way as to minimize practice interruption and support changes in health status among patients who have not previously had access to weight management services.

The Wellness Connect project has demonstrated that telehealth technologies can be used to effectively provide weight management interventions. Data show that patients achieved statistically and clinically significant weight loss (>4.0\%). Provider feedback and engagement have been strong and has resulted in additional collaboration across the state to address unresolved issues of insurance coverage and payment parity. Several knowledge points have emerged from the project. First, there is importance in being able to deploy a multi-disciplinary team including clinicians (which includes an interdisciplinary team of clinicians), technologists and educators. Improving the access to care for patients involves a great deal of education and practice refinement in addition to technical support. Next, telehealth technology is only problematic in that it expands services and processes into new areas. Results show that providers struggle less with technology than was expected and more with traditional practice issues like sending out patient reminders. Additionally, the program is highly dependent on nursing/medical staff for successful patient recruitment and retention.

The strength of individual clinic outcomes appears to align with the engagement of the physician champion and consistency of the nurse facilitator. Likewise, mobile apps that meet the needs of rural primary care providers for data collection, review and care coordination are important to fully support telehealth programs. Managing data using custom apps that are secure, compliant and tailored to the needs of the quality improvement project are imperative when working with numerous practices over geographic distances. Finally, insurers continue to provide little to no coverage of appropriate obesity treatment. Even insurers with perhaps the best obesity coverage rarely cover treatments/programs that conform to the USPSTF recommendation. The Wellness Connect project is demonstrating that, through academic and community provider partnerships, telehealth technology can be used to improve population health in rural communities, generally, and effectively deliver evidence-based obesity treatment, specifically.

\section{Objectives}

1. Describe the use of telehealth technologies and processes to support weight management services in partnership with primary care providers

2. Apply new models of telehealth and m-Health technologies to facilitate population health

3. Understand the processes required for successful implementation of evidenced-based weight management intervention

\section{P41 EFFECT OF TELEMANAGEMENT IN PATIENTS DISCHARGED TO A SKILLED NURSING FACILITY AFTER A HEART FAILURE HOSPITALIZATION \\ PRESENTER: \\ Santosh G. Menon, MD, Medical Director \\ Carl and Edyth Lindner Heart Failure Treatment Center, The Christ Hospital, Cincinnati, $\mathrm{OH}$ \\ Nancy T. Rector, BS, Medical Technology, Telemanagement The Christ Hospital, Cincinnati, $\mathrm{OH}$}

Purpose: The purpose of this study was to investigate if telemanagement of patients discharged to a skilled nursing facility (SNF) after a hospitalization for heart failure would reduce hospital readmissions and improve patient selfcare knowledge and satisfaction.

Background: Heart Failure - (1) In 2013, over one million patients were admitted to hospitals in the United States for HF, and 25\% of these patients were readmitted within 30 days (Hummel et al., 2014). (2) "Heart failure is also the most common reason for readmissions after any hospital discharge, regardless of the reason for the original hospitalization" (Au et al., 2012, p. 365). (3) Institutional data reveals patients discharged to a skilled nursing facility (SNF) have a higher risk of readmission compared with patients discharged home or with home health care (HHC). Telemedicine - Telemedicine has rapidly gained traction as a healthcare delivery model which has shown to decrease hospitalizations (Bashshur et al., 2014).

Methods: Setting - (1) Patients discharged from The Christ Hospital (TCH), a 555- bed, not-for-profit acute care tertiary facility located in Cincinnati, Ohio 
to Brookwood Retirement Community for skilled level of care. (2) Patients were roomed in same hallway at SNF and with consistent staffing. (3) Patients transitioning home after SNF stay utilized Stay Well Home Health Agency. Sample - (1) Patient eligibility - (a) Admitted with a primary or secondary diagnosis of heart failure, (b) Require skilled level of care after discharge, and (c) Established with TCH cardiology group. Data Collection - (1) Readmission rates: utilizing electronic health record review and SNF/HHC report. (2) $\mathrm{Pa}-$ tient self-care knowledge and satisfaction: using questionnaire. Intervention (1) Interactive telemanagement video sessions with Heart Failure Clinic MD or NP facilitated by SNF/HHC. (2) After transition from hospital to SNF, and from SNF to HHC. (3) As needed for change in patient condition. (4) HF education and monitoring. (5) Duration - Up to 30 days at SNF with an additional 30 days once discharged home with HHC. Technology - (1) Biopatch Device - (a) Heart Rate, (b) Respiration Rate, (c) EKG, (d) Posture/Position, and (e) Activity minutes; (2) Integrated Ancillary Devices for Zephyr Life Home System - (a) Blood pressure, (b) Body weight, and (c) Oxygen saturation; (3) Other Devices (a) $3 \mathrm{M}^{\mathrm{TM}}$ Littmann ${ }^{\circledR}$ TeleSteth ${ }^{\mathrm{TM}}$ Blue Tooth stethoscope and (b) Abbott i-Stat for point-of-care lab testing. Clinical interventions available (SNF) - (1) Change in medication administration, (2) Onsite Moderately Complex Laboratory Testing, (3) B-type natriuretic peptide (BNP), (4) Chem 8+: Sodium, Potassium, Chloride, TCO2, Anion Gap, Ionized Calcium, Glucose, BUN/Urea, Creatinine, Hematocrit, Hemoglobin, (5) Radiology testing, (6) Consult for Dietitian, (7) Respiratory Therapy, and (8) 12-lead EKG.

Results: (1) 42 patients enrolled to date, with goal enrollment of 60 by February 1, 2015, (2) 7 patients readmitted within 30 days of hospital discharge (16.7\%), (3) Only one patient readmitted for HF, (4) All patients who readmitted were New York Heart Association (NYHA) Class IV, (5) Average length of stay during initial hospitalization was 9.3 days, 26 days at SNF, (6) Average of one telemanagement session per patient at SNF, one at home, (7) Average length of session: 16 minutes, (8) Average number of cardiac interventions (medication) per patient at SNF: 8, and (9) There was one expected death. Baseline Sample Characteristics - (1) Demonstrated interaction between patient and other family members via use of the tablet, (2) Patient comments: "thankful," "excited," "did not want it to end", (3) Identified new diagnoses of atrial fibrillation and pneumonia through video session assessment, and (4) Replaced 7 day physician follow-up appointment with Vidyo (Virtual Care Works) appointment at an increased rate as the study progressed. Conclusion: (1) Comprehensive telemanagement of heart failure patients discharged to a skilled nursing facility is feasible and appears to a have a favorable effect on 30-day all cause readmissions. (2) Technology was well-received with high patient satisfaction and ease of use reported in elderly patients with HF. (3) Telemanagement is a new method used to achieve reduced readmission rates even in high risk populations admitted to SNFs. (4) This technology has the potential to enhance communication between the discharging team, the patient, the skilled nursing facility, home care agency, and heart failure team.

\section{References}

1. Au A, McAlister F, Bakal J, Ezekowitz J, Kaul P, Van Walraven C. Predicting the risk of unplanned readmission or death within 30 days of discharge after a heart failure hospitalization. Am Heart J 2012; 164(3):365-72.

2. Bashshur R, Shannon G, Smith B, et al. The empirical foundations of telemedicine interventions for chronic disease management. Telemed $J$ E Health 2014; 20(9):769-800.

3. Centers for Disease Control and Prevention (2013). Division of heart disease and stroke prevention. Retrieved from http://www.cdc.gov/ dhdsp/data_statistics/fact_sheets/docs/fs_heart_failure.pdf

4. Hummel S, Katrapati P, Gillespie W, DeFranco A, Koelling T. Impact of prior admissions on 30-day readmissions in Medicare heart failure inpatients. Mayo Clin Proc 2014; 89(5):623-30.

\section{Objectives}

1. Enhance communication between the discharging team, the patient, the skilled nursing facility, home care agency, and heart failure team to identify early detection of signs and symptoms that may indicate an exacerbation of CHF utilizing telehealth

2. Optimize treatment within the facility or at home to reduce readmissions to the hospital

3. Facilitate an easy and safe transition of the patient from the hospital to the skilled nursing facility and then to community based self-management through the use of telemedicine and point of care laboratory testing

\section{Track: Mental Health}

\section{TUESDAY, MAY 17, 2016}

10:35 AM-11:00 AM Tuesday, May 17, 2016

\section{EPOSTERS: MEET THE AUTHOR IN THE ATA EXPERIENCE ZONE/EXHIBIT HALL}

\section{P48 SUCCESSFULlY UTILIZING A BEHAVIORAL HEALTH SERVICE LINE AND LEAN PRACTICES TO REDUCE ED LENGTH OF STAY FOR THE VIRTUAL PSYCHIATRIC PATIENT}

\section{PRESENTER}

Amy K. Barrett, LPC, Program Coordinator

Carolina Healthcare System, Weddington, NC

North Carolina has had a dramatic increase in psychiatric patients presented to medical emergency departments with behavioral health needs. CHS is dedicated to improving the access and quality of care to the psychiatric population. Through the use of innovative technology, virtual teams, a service line, and Lean practices, we have succeeded in improving our process and addressing the specific needs of this population.

Clinical barriers for psychiatric patients in medical ED's include variation in care and documentation, decreased quality of care, chaotic environments increasing stress, and increased demand on nursing resources. Our information services team has created a multi-facility electronic tracking queue which prevents numerous phone calls to coordinate care and provide status updates. It also supports decreased patient length of stay and increases productivity of clinicians and providers. Considerable communication challenges are embedded in this professional collaboration between the ED and the behavioral health service line to coordinate and

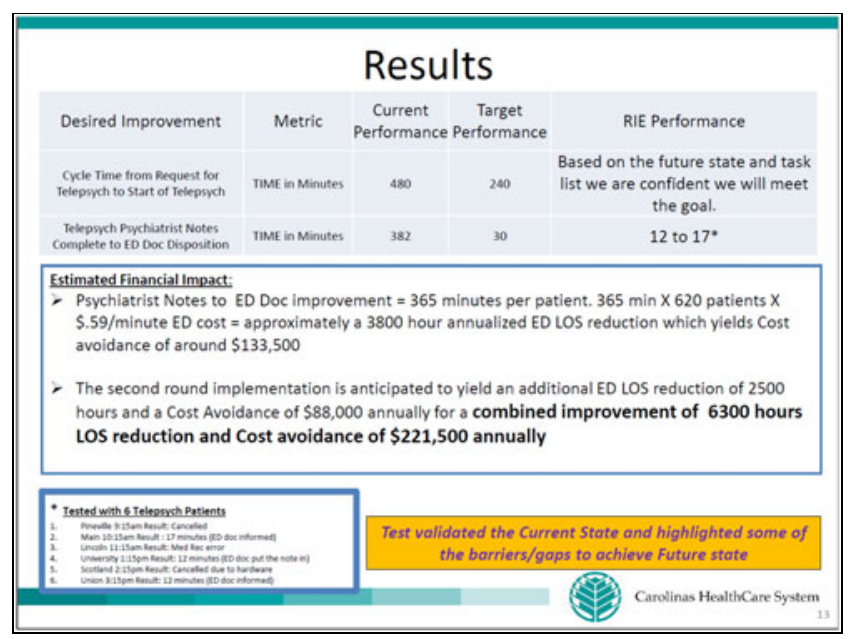


actualize a virtual consult result. Lean Value Stream Mapping and Rapid Improvement Events assist in the creation of an efficient, patient-centered, standardized virtual consulting process including the placement of patients requiring inpatient stabilization. Streamlining the psychiatric consultations process has enhanced patient treatment while increasing patient and employee satisfaction.

Lean techniques improve patient care by reducing delays, redesigning and standardizing the processes, and staffing to demand. The scientific method is used to solve problems in conjunction with the daily Huddle and direct observations. The Lean focus supports the reduction of patient length of stay, improves quality of care, and increases teammate engagement.

1. Venkat, Subbu, Navarro, Fernando, Thorsen, Dawn, Sparks, Wayne, Barrett, Amy, Metts, Michael, King, Stephanie, Zazzaro, Christine, Carroll, Anna, Cornellier, Kim, Callaway, Jennifer, DeSear, Nathan, Sawyer, Andrew, Cousino, Craig, and Smith, Stuart. (2014) RIE Report Out: Psychiatric Patient Length of Stay Pineville ED. Charlotte, NC: Carolinas Healthcare System.

\section{Objectives}

1. Identify hurdles in providing virtual care in acute medical emergency departments

2. Demonstrate how the use of LEAN resources assists in the elimination of waste and standardization of care

3. Explain the advantages of utilizing a Behavioral Health service line to improve the quality of care and efficiency of virtual psych consults

\section{TUESDAY, MAY 17, 2016}

10:35 AM-11:00 AM Tuesday, May 17, 2016

\section{EPOSTERS: MEET THE AUTHOR IN THE ATA EXPERIENCE ZONE/EXHIBIT HALL}

\section{P49 FEASIBILITY OF DELIVERING BEHAVIORAL SLEEP INTERVENTIONS THROUGH MOBILE HEALTH (M-HEALTH) APPLICATION FOR MILITARY POPULATIONS}

\section{PRESENTER:}

Andi Saptono, PhD, Assistant Professor

University of Pittsburgh, PA

Introduction: Sleep disturbances are prevalent among Active Duty Service Members (ADSM) and Veterans, and threaten psychological health, resilience, and the military mission. Hypnotics remain the most common sleep treatments among ADSM and Veterans, despite substantial concerns regarding their safety (particularly in operational settings), side effects, and potential for abuse. Behavioral sleep treatments have been shown to be safe and effective, and are associated with durable improvements. However, the use of evidence-based, resilience-focused, behavioral treatments to promote healthy sleep pre-deployment and to treat sleep disturbances post-deployment remains scarce among ADSM, Veterans, and the clinicians who serve them. Mobile health (m-Health) has the potential to increase access to these behavioral treatments and to improve their effectiveness by tailoring and adapting the sleep interventions based on each individual's needs.

Methods: We developed a cross-platform m-Health solution called iREST (interactive Resilience Enhancing Sleep Tactics) which consists of (1) a smartphone application (app) that records sleep data, shows feedbacks and related education materials, and provides cues and notifications (2) a Web-based portal that allows therapists to monitor sleep information, to prescribe treatment, and to engage participants in real-time via secure messages, (3) wearable sensors integration, and (4) a communication protocol that allows real-time bidirectional data exchange between the all components. A pilot study with 12 ADSM and Veterans examined the feasibility and usability of $i$ REST. The System Usability Score (SUS) and The Telerehabilitation Usability Questionnaire (TUQ) were used to measure iREST app usability. Participants completed Epworth Sleepiness Scale (ESS) and Insomnia Severity Index (ISI) prior and after four weeks of using the app.

Results: $i$ REST was found to be capable of supporting behavioral sleep treatments in ADSM and Veterans. Participants rated the app as highly usable with SUS mean score of $81.3(S D=13.9)$; score above a 68 would be considered above average. On the TUQ, participants reported high level of ease of use (4.25 out of 5), satisfaction (4.00 out of 5) and interface quality (3.93 out of 5). Before and after intervention test shows significant decrease in participant ESS score, a change of $-1.75(p<0.05)$, and a change of $-8.75(p<0.05)$ in their insomnia index (ISI). Five of the 6 participants (83\%) who have now completed the 4-week trial period fully remitted from insomnia.

Conclusions: A smartphone app is feasible within sleep intervention for ADSM and Veterans. Integrating an $\mathrm{m}$-Health platform may facilitate involvement in treatment and dissemination of effective tools in military and civilian samples.

\section{Objectives}

1. Recognize challenges in delivering behavioral sleep treatment

2. Identify technology and platform available to address those challenges

3. Discuss feasibility of $\mathrm{m}$-Health in delivering behavioral treatment

\section{P51 RECOVERY SUPPORT TECHNOLOGIES: PIONEERING NEW MODELS OF SUBSTANCE ABUSE RECOVERY SERVICES}

\section{PRESENTER:}

Joyce A. Hartje, PhD, Project Manager - Evaluation Research

University of Nevada, Reno, $N V$

Often what propels individuals toward seeking recovery services are a series of cascading problems associated with substance use (e.g., job insecurity, difficulties with family and social ties, co-occurring health issues, housing instability, legal difficulties, etc.). For this reason, availability of immediate services is best, as many studies show that delays in treatment access are associated with lower rates of recovery. Earlier definitions of recovery focused on abstinence and sobriety. However, most individuals with "lived experience," addiction treatment professionals, and peer support specialists agree that while recovery may include abstinence/sobriety, it also includes a focus on health and community. In 2012, the Substance Abuse and Mental Health Services Administration (SAMHSA) adopted a definition of recovery as being "a process of change through which individuals improve their health and wellness, live a self-directed life, and strive to reach their full potential."

For many individuals, finding a source of support is an essential need that must be met before recovery progression can occur. Accordingly, there are many highly personal and varied pathways to recovery, and recovery support services help people enter into and navigate systems of care, remove barriers to recovery, stay engaged in the recovery process, and live full lives in communities of their choice. These services can be provided through treatment and community-based programs by behavioral health care providers, peer providers, family members, friends and social networks, the faith community, and people with experience in recovery. However, the overwhelming failure of traditional models of treatment to meet the needs of so many with substance use disorders has led to the development of innovative approaches to treating and promoting recovery among those struggling with addiction.

New approaches, such as recovery support technologies, are a response to the intractable barriers that keep 20 million people from accessing recovery support. The pervasive use of computers, mobile phones, and other forms of technology points to its use as a critical means of reaching the populations who are at highest risk of needing but unable to attain substance use treatment and recovery services. Thus, technology offers one more avenue by which the behavioral health field can provide recovery support services, thereby increasing recovery participation and decreasing the likelihood of relapse. 


\section{ePOSTER PRESENTATIONS ABSTRACTS}

However, despite this move toward using technology to expand access to recovery services, there is no guarantee that staff and/or customers will embrace using that technology. Research has examined the complexity of individuals' relationships with technological innovation, identifying several paradoxes experienced when dealing with new technologies. Several theories have been used to examine: the choices an individual makes to accept or reject a particular innovation and the extent to which that innovation is integrated into the appropriate context (adoption); how an innovation spreads through a population (diffusion); and beliefs and attitudes that are formed over time that may influence decisions about the innovations adopted or rejected. This presentation will first identify some of the barriers to accessing recovery support services, discuss why mobile technology and the internet play a role in reducing the barriers, review the research on the benefits of using technology for recovery support, and showcase several types of technology that can be used to help deliver recovery support services, improve disease management, and compliment behavioral health treatment services. In addition, the presenter will discuss results of a recent study that suggest positive correlations between individuals' attitudes toward using technology and their propensity to deliver substance abuse recovery services using telehealth technologies.

1. Garner BR, Scott CK, Dennis ML, Funk RR. The relationship between recovery and health-related quality of life. J Subst Abuse Treat 2014; 47(4):293-98.

2. Laudet $A B$, Humphreys $K$. Promoting recovery in an evolving policy context: What do we know and what do we need to know about recovery support services? J Subst Abuse Treat 2013; 45(1):126-33.

3. Ratchford M, Barnhart M. Development and validation of the Technology Adoption Propensity (TAP) Index. J Bus Res, 2013; 65(8):1209-215.

4. SAMHSA. Recovery and Recovery Support. Retrieved June 2015 from http://www.samhsa.gov/recovery

\section{Objectives}

1. Following this presentation, participants will be able to define recovery and recovery support services

2. Following this presentation, participants will be able to identify three barriers to traditional recovery support service delivery

3. Following this presentation, participants will be able to explain how technology can address barriers to accessing recovery support services

\section{P52 THE TELEMENTAL HEALTH REVOLUTION: DISPARITIES} IN TRAINING FUTURE CLINICIANS TO USE TECHNOLOGY IN PRACTICE

\section{PRESENTER:}

Hannah M. Kersting, BA, Doctoral Student

Spalding University, Louisville, $K Y$

Introduction: The field of psychology's involvement with information and communication technology (ICT) to provide clinical services lags behind that of other healthcare disciplines (Maheu et al., 2004) and such disparity extends to graduate training program curricula. As sophistication of and access to technology evolve, more individuals with psychological concerns may seek mental health consultations and treatment via technology media (Maheu et al.). Despite the continued development of these applications, mental health clinicians are lacking in the training to utilize these programs effectively (Maheu et al., 2012). For example, research suggests that agencies are under utilizing telemental health services (e.g. focus is primarily on medication management). Additionally, practitioners and students are ill prepared to provide such services given their training and education in this subspecialty (Jameson et al., 2011; Maheu et al.). As many other researchers have stated, telemental health training is vital for preparing students to deliver treatment in the modern era (Belar, 1998; Glueckauf et al., 2003; Glueckauf \& Lustria, 2008; Maheu et al., 2004; Maheu, Whitten, \& Allen, 2001) even though only a few graduate and postgraduate programs currently include this training in their curricula (McKay et al., 2013). The current study was part of a larger mixed methods design project focusing specifically on the experiences with and utilization of telemental health services while in graduate training. Mentioned concerns with privacy, government/ ethical regulations, and the intricacies of the unfamiliar technology must be alleviated through comprehensive expert training. The companies that produce ICT along with clinical psychology graduate programs must unite to create comprehensive trainings that address graduate students' trepidation with telemental health. Until then, the field of psychology, with its resistance to utilizing these revolutionary technologies, will continue to fall behind other medical communities in its ability to enhance the wellbeing of underserved populations.

Methods: Participants - Masters and doctoral graduate students $(N=75)$ in APA approved clinical and counseling psychology programs were recruited. Students ranging from first year masters through students on doctoral internships responded. The majority of participants identified as Caucasian $(\mathrm{n}=65,86.7 \%)$ and women $(\mathrm{n}=64,85.3 \%)$. Respondents ranged in age from 22-44. Measures - As part of a larger study, participants were asked to complete a demographic questionnaire and a brief survey to assess graduate students' knowledge of, attitudes towards, and experiences with telemental health and current training opportunities in graduate psychology programs. Procedures - Recruitment was conducted by sending an IRB approved email to directors of clinical training and LISTSERV moderators of relevant divisions of the American Psychological Association.

Results: Results of the current study suggest that graduate students $(\mathrm{N}=75)$ are minimally exposed to the concepts surrounding telemental health. Only $50.82 \%$ of respondents reported being exposed to the term "telepsychology" during their undergraduate and graduate training programs. Of those who reported using ICT, only $22.67 \%$ of participants indicated ever using technology to deliver services to populations in diverse settings. Additionally, $76 \%$ of respondents noted a moderate to high level of dissatisfaction with their current lack of training in how to practically implement these technologies in their clinical practice. Similar themes were revealed through the qualitative exploration portion of the study, with participants noting high levels of discomfort with their lack of expert training and with the technologies in general. While participants mentioned the necessity of using telemental health technologies to comprehensively serve formerly unreachable populations, many reported apprehension with the field's ability to incorporate efficacious technology programs, universal confidentiality standards, and proper training with the high standards of care to which patients have become accustomed.

Conclusions: Burgeoning research touts a need for training programs to begin including supervised experiences for students to increase an understanding of telemental health, exposure to training opportunities, as well as guidance around navigating the parameters of such practice. Joint efforts are needed between graduate training programs and experts in telemental health practice to help future clinicians become comfortable in utilizing the software programs and technology in training as well as moderating the use of such services in supervised clinical practice. The mutual expertise could allow for the sharing of unique competencies to further promote the use of telemental health programs with clients and to help assuage students' aforementioned concerns. Additional future implications as well as study limitations will be discussed.

1. Glueckauf RL, Pickett TC, Ketterson TU, Loomis JS, Rozensky RH. Preparation for the delivery of telehealth services: A self-study framework for expansion of practice. Professional Psychology: Research and Practice 2003; 34(2):159-63.

2. Maheu MM, Whitten P, Allen A. (2001). E-Health, telehealth, and telemedicine: A guide to start-up and success. San Francisco: Jossey-Bass.

3. Maheu MM, McMenamin J. (in press). Telepsychology best practices \& professional training. In G. Koocher, J. Norcross, \& B. Greene (Eds.), Psychologists' Desk Reference. New York, NY: Oxford University Press.

\section{Objectives}

1. Demonstrate awareness of the disparities in training future clinicians to use technology in practice 
2. Identify why there is a lack of technology being utilized in the mental health field

3. Discover the needs of psychologist regarding their abilities to deliver services and consultations via technology

\section{Track: Pediatrics}

\section{TUESDAY, MAY 17, 2016}

10:10 AM-10:35 AM Tuesday, May 17, 2016

\section{EPOSTERS: MEET THE AUTHORS IN THE ATA EXPERIENCE ZONE/EXHIBIT HALL}

\section{P42 ACCURACY OF PEDIATRIC TELE-RHEUMATOLOGY PHYSICAL EXAMINATION FINDINGS AND DIAGNOSTIC CONCORDANCE AT A DISTANCE}

\section{PRESENTER:}

Michael Henrickson, MD, MPH, Associate Professor

Cincinnati Children's Hospital Medical Center, Cincinnati, $\mathrm{OH}$

Introduction: Telemedicine (TM) offers a strategic means of extending limited clinical pediatric rheumatology (PR) workforce capacity to improve access to care for patients in remote locations. Tele-rheumatology (TR) often depends on a remote site provider to convey physical examination (PE) findings. While adult TR has diagnostic accuracy of 97\% compared to inperson consultations, there are no published data for pediatric TR PE or diagnostic accuracy. This cross-sectional observational study assessed the accuracy of PE findings in 10 new PR TR consultations. Pragmatically, we envision a service delivery model involving initially inexperienced musculoskeletal examiners at remote locations. Hence, our aim was to determine the reproducibility of PE findings and diagnostic agreement. The primary outcome was comparison of the physician TR and remote examiner PE findings, and diagnostic concordance.

Methods: The physician conducted the interview within our hospital at a dedicated TM suite, then guided and documented the remote provider's PE. This examiner, a developmental pediatrics physical therapist, was physically located with the patient at our institution's PR clinic. We deliberately sought an examiner naïve to PR musculoskeletal examination. Knowing PE skills improve with repetition, we restricted the study to 10 patients to limit this effect. Patient selection focused on ensuring a variety of presenting complaints and ages. The initial 30\% knew they had TR consultation scheduled; the remainder were unaware. We sought parental TM satisfaction after each session via on-line survey and/or in-person interviews. We used a Likert scale to determine the impact revised PE findings had on clinical decision-making.

Results: We identified 26 PE errors in this cohort of broad age range (14 months to 16 years). The table summarizes concordance. PE errors mainly involved biomechanical disorders with or without generalized hypermobility $(7,2)$ and musculoskeletal abnormalities (5). Weighted averages for diagnostic concordance varied: overall (37\%), biomechanical disorders (31\%), inflammatory diseases (83\%) and pain amplification (0\%). The practical impact on decision-making (20\% none, 30\% minimal, 50\% moderate, 0\% marked) affected 8 patients: physiotherapy orders (5), and one patient each for lab/x-ray, medication options, and additional consultation. Completed surveys (50\%) indicated 100\% agreed or strongly agreed with using TR again, with overall satisfaction of $100 \%$.

Conclusions: Pediatric TR requires a remote site examiner trained in musculoskeletal PE with an emphasis on biomechanical abnormalities

\begin{tabular}{|c|c|c|c|}
\hline \multicolumn{4}{|c|}{ CONCORDANCE FOR PE FINDINGS AND DIAGNOSIS } \\
\hline & $\begin{array}{c}\text { PE } \\
\text { FINDINGS } \\
\text { IDENTIFIED } \\
\text { MD ONLY }\end{array}$ & $\begin{array}{l}\text { PE FINDINGS } \\
\text { IDENTIFIED } \\
\text { BOTH } \\
\text { EXAMINERS } \\
\text { (PT \& MD) }\end{array}$ & $\begin{array}{c}\text { DIAGNOSTIC } \\
\text { CONCORDANCE } \\
(\%)\end{array}$ \\
\hline Acrocyanosis & & 1 & 100 \\
\hline Complex regional pain syndrome & 1 & 0 & 0 \\
\hline $\begin{array}{l}\text { Hypermobile type Ehlers Danlos } \\
\text { syndrome }\end{array}$ & 2 & 4 & 67 \\
\hline $\begin{array}{l}\text { Oligoarticular JIA (TMJs, } \\
\text { peripheral joints) }\end{array}$ & & 2 & 100 \\
\hline $\begin{array}{l}\text { Other biomechanical disorders } \\
\text { (iliotibial band, ligament laxity, } \\
\text { patellofemoral } \\
\text { instability) }\end{array}$ & 7 & 0 & 0 \\
\hline $\begin{array}{l}\text { Other musculoskeletal (flexion } \\
\text { contracture, joint crepitus } \\
\text { or tenderness, malocclusion, } \\
\text { and muscle atrophy) }\end{array}$ & 5 & 0 & 0 \\
\hline $\begin{array}{l}\text { Periodic fever } \\
\text { syndrome }\end{array}$ & & 2 & 100 \\
\hline Reactive arthropathy & 1 & 1 & 50 \\
\hline
\end{tabular}

which are prevalent in PR. Concerns about reliably identifying juvenile arthritis do not appear to be a major risk of TR, although errors did occur in detecting all involved joints. Future application of pediatric tele-rheumatology will need to address the identified training gap or create technologies which can facilitate remote musculoskeletal examination by the hub physician. Pediatric TR can provide access to clinical care with high levels of satisfaction.

1. Leggett P, Graham L, Steele K, Gilliland A, Stevenson M, O'Reilly D, Wootton R, Taggart A. Telerheumatology _ diagnostic accuracy and acceptability to patient, specialist, and general practitioner. $\mathrm{Br} J$ Gen Pract 2001; 51(470):746-8.

\section{Objectives}

1. Compare hub physician and remote examiner physical examination findings by tele-rheumatology

2. Demonstrate the accuracy and reproducibility of physical examination findings identified during pediatric tele-rheumatology new encounters

3. Assess the diagnostic accuracy of pediatric tele-rheumatology

\section{P43 TELE-EARLY INTERVENTION PARTNERSHIPS AS A STATEWIDE STRATEGY TO INCREASE ACCESS TO SERVICES FOR TODDLERS WITH AUTISM SPECTRUM DISORDER AND THEIR FAMILIES}

\section{PRESENTER:}

Marilyn Espe-Sherwindt, PhD, Director, Family Child Learning Center Akron Children's Hospital, Tallmadge, $\mathrm{OH}$

Autism Spectrum Disorder (ASD) is the fastest growing developmental disorder in the United States. Currently, one out of every 68 children has been identified with ASD (CDC, 2015); the prevalence has more than doubled since 2002. Since signs of autism appear early, a diagnosis can often be made before age 


\section{ePOSTER PRESENTATIONS ABSTRACTS}

2 (even as early as 14 months), and a diagnosis of ASD in toddlers has been shown to be stable, valid and reliable (Kleinman et al., 2008; Lord et al., 2006). Research suggests that programs for children under three diagnosed with ASD should include parent involvement and engagement with their child, and that positive outcomes are related to (1) the intensity of that engagement and (2) starting as early as possible (Dawson et al., 2010; Rogers et al., 2012). Furthermore, parents are able to learn, implement, and maintain engagement strategies throughout the day in daily routine interactions with their toddler with ASD (Dawson et al., 2010; Hardan et al., 2015; Vismara, Colombi \& Rogers, 2009; Wetherby et al., 2014).

Toddlers diagnosed with ASD typically receive services through a state's system of early intervention (EI) system. Even though Ohio is now better able to make earlier and accurate diagnosis of ASD, not all families have access to EI providers who are specialized and highly trained to work with toddlers newly diagnosed with ASD. In addition, other factors contribute to the inability of some families to get the resources they need: (1) the recent economic ups and downs have had a devastating impact on early intervention in many Ohio counties; (2) while Ohio has a highway system that efficiently connects most parts of the state, the southeast region of the state (a land mass as large as the state of Connecticut), lacks a good highway system, making accessibility to both jobs and health care difficult.

Technology can provide a unique approach to meet these early intervention personnel, fiscal and geographic challenges (Baharav \& Reiser, 2010; Hamren \& Quigley, 2012; Olsen, Fiechtl \& Rule, 2012). The Family Child Learning Center (FCLC) is the early intervention demonstration, teaching and research center of Akron Children's Hospital. As a collaborative partner in a three-year (2011-2014) federal HRSA State Implementation Grant for Improving Services and Supports for Children with ASD and other Disabilities awarded to the state of Ohio, FCLC developed and tested a pilot program of tele-early intervention partnerships with nine counties across the state of Ohio who did not have providers trained to work with toddlers with ASD and their families.

In this model, FCLC used the hospital's videoconferencing system to conduct virtual early intervention visits in the homes of families with toddlers with ASD. On one end was an FCLC early interventionist trained to use evidence-based approaches with families of toddlers with ASD; on the other end, a local service provider (who had received introductory "virtual" training from FCLC in the intervention approach and the technology) carried a laptop, speakerphone, webcam and mobile broadband into the family's home and participated with the family and child in the virtual home visit. The home visits were recorded and made available for later viewing by caregivers who could not be present.

As a result, all family members and the local professionals were being coached in how to implement intervention strategies. Some anticipated and unanticipated technological barriers emerged at various points throughout the project, e.g., bandwidth, HIPAA compliance, and lack of confidence on the part of the professionals at both ends. Nevertheless, project data demonstrated that both families and local professionals experienced positive outcomes with "virtual" home visiting:

- Tele-early intervention virtual home visits were initiated on bi-weekly basis for 21 toddlers (16 males \& 5 females, average age at ASD Dx of 2.29 years) and their families;

- $100 \%$ of the families who received ongoing services and transitioned out of EI before the grant ended would recommend tele-early intervention to another family;

- Qualitative analysis of data from a focus group of the local service providers indicated that through their participation in the virtual home visits, they had increased their understanding of ASD and had learned intervention strategies that they were able to generalize to other families.

- A McNemar's test comparison of the submitted pre-post family ratings of their own knowledge and skills demonstrated significant positive changes $(p<.001)$, similar to pre-post ratings of families who had received face-to-face EI at FCLC.

Given the success of the pilot program, Akron Children's Hospital sought funding to continue and expand the tele-early intervention partnerships.
Currently FCLC is testing a virtual version of CONNECTIONS (a short-term intense intervention program for toddlers with ASD developed at FCLC) and comparing its outcomes to the family and child outcomes already documented in the face-to-face program (parent-child interactions, parental self-confidence, and family quality of life).

1. Baharav E, Reiser C. Using telepractice in parent training in early autism. Telemed J E Health 2010; 16(6):727-31.

2. Centers for Disease Control and Prevention. (2015). Autism Spectrum Disorder (ASD). Retrieved 7/10/15 from http://www.cdc.gov/ncbddd/ autism/data.html

3. Olsen C, Fiechtl B, Rule S. An evaluation of virtual home visits in early intervention: Feasibility of "virtual intervention." The Volta Review 2012; 112(3):267-81.

4. Rogers SJ, Estes A, Lord C, Vismara L, Winter J, Fitzpatrick A, Guo M, Dawson G. (2012). Effects of a brief Early Start Denver Model based parent intervention on toddlers at risk for autism spectrum disorders: a randomized controlled trial. J Am Acad Child Adolesc Psychiatry 2012; 51(10):1052-65.

5. Wetherby AM, Guthrie W, Woods J, Schatschneider C, Holland RD, Morgan L, Lord C. Parent-implemented social intervention for toddlers with autism: An RCT. Pediatric 2014; 134(6):1084-93.

\section{Objectives}

1. Identify the statewide barriers that families face in accessing evidencebased early intervention for their toddler with autism spectrum disorder

2. Discuss the process (challenges and successes) of developing tele-early intervention partnerships with resource-poor areas of the state as a strategy to deliver early intervention services to families in their homes

3. Describe the impacts of the tele-early intervention partnerships on families, their children, and the local service providers

\section{P44 TELEPRACTICE SERVICE DELIVERY FOR CHILDREN'S COMMUNICATIVE DISORDERS: IS IT EFFECTIVE?}

\section{PRESENTER:}

Kathy L. Coufal, PhD, Professor

Wichita State University, Wichita, KS

Introduction: The use of telepractice to provide speech/language services has become a more recognized option for service delivery to children in schools. There are companies now offering services to people that otherwise would not be possible. A high demand for services and a shortage of qualified professionals are persistent problems. There exists inaccessible rural areas and cities where caseloads for Speech-Language Pathologists (SLPs) are too high to manage appropriately. It has been shown that, for some, quality care is nonexistent due to unavailability of qualified professionals (Wilson et al., 2004). Access to care is a major challenge in the provision of speech/language services, and the introduction of telepractice may alleviate some barriers. According to the American Speech-Language-Hearing Association's (ASHA) position statement, "telepractice offers the potential to extend clinical services to remote, rural, and underserved populations and to culturally and linguistically diverse populations" (ASHA, 2005). The question remains, however, whether services provided through telepractice are as effective as traditional, face-to-face service delivery.

Comparing Service Deliveries: It is critical that a common metric be used to allow for comparisons between face-to-face services and telepractice. ASHA developed the National Outcomes Measurement System (NOMS), a voluntary data collection system, to standardize and quantify reportable outcomes for speech-language pathology services. NOMS allows outcomes-based reporting on ASHA's Functional Communication Measures (FCMs), which are "a series of disorder-specific, seven-point rating scales designed to describe the change in an individual's functional communication and/or swallowing ability over time" (ASHA, 2015). Clients are assigned initial and final scores (on a scale of 1-7, with lower scores meaning less functional ability), from which a change 


\section{ePOSTER PRESENTATIONS ABSTRACTS}

score can be calculated. Thus, the NOMS FCMs provides a common rubric and scoring system used to compare outcomes between face-to-face and telepractice intervention.

Purpose: The purpose of this retrospective investigation was to determine if there is a difference in the reported outcomes for children's speech sound production by comparing face-to-face service delivery (using the ASHA NOMS database) and telepractice service delivery (using a proprietary database provided by a private e-learning provider). Given that no reports of such comparisons exist, this study was exploratory in nature, as opposed to testing a directional hypothesis.

IRB Approval and Permissions: This study was approved by the Institutional Review Board at Wichita State University. Permission to use the datasets was given by both the ASHA and the private e-learning provider.

Case Selection: Inclusion in the analysis were the reported scores of children served in school settings, from any geographic region within the U.S., and meeting the following three selection criteria: (1) the children received therapy for the category "speech sound production," with no cooccurring communication disorder, (2) they were between six and nine years of age, corresponding to early elementary-school grades, and (3) the length of the children's treatment was four to nine months, which was the typical length of services during the school year. The ASHA NOMS dataset contained 1,870 cases, the e-learning provider's dataset had 588. For the current analysis, 500 cases from each dataset were randomly selected $(n=1,000)$.

Analysis: The dependent variable for this analysis was the change score between the initial and final NOMS scores. Because the NOMS scoring uses a seven-point scale, the range of change scores is limited. Moreover, because therapeutic improvement is usually a gradual process, change scores are atypically distributed, having high concentrations of scores ranging from 0 to 2 points. For both of these reasons, non-parametric analyses were used to test for differences between group medians and rank scores between the groups.

Results: A Mann-Whitney U Test revealed no significant differences in the change scores between the face-to-face group (Median $=1, n=500$ ) and the telepractice group (Median $=1, \mathrm{n}=500$ ), $U=117147.5, z=-1.784, p=0.074$, $r=0.079$.

Discussion: The results of this preliminary analysis of one Functional Communication Measures category (speech sound disorders) in a select cohort of early elementary-school children suggest there is no significant difference in treatment outcomes between traditional, face-to-face speech/ language intervention and services provided through telepractice. Moreover, because the NOMS is designed to reflect functional communication outcomes, the results of this cohort comparison support the use of telepractice as an effective service delivery option, with outcomes that do not differ statistically or functionally from traditional, face-to-face intervention. Given that telepractice and traditional service delivery did not result in meaningful differences in outcomes, the problem of access to qualified service providers and quality interventions for children with speech-sound disorders may be ameliorated through telepractice. The substantial number of children in the early grades who demonstrate speech-sound disorders often dominate the caseloads of school-based speech-language pathologists. If these children can successfully improve through services provided via telepractice, a gap in current practices can be addressed as an increasing number of telepractice providers work with schools. American Speech-LanguageHearing Association. (2005). Speech-language pathologists providing clinical services via telepractice [Position Statement]. Retrieved from http://www .asha.org/docs/html/PS2005-00116.html

1. American Speech-Language-Hearing Association. (2015). ASHA's National Outcomes Measurement System (NOMS). Retrieved from http:// www.asha.org/NOMS/

2. Wilson L, Onslow M, Lincoln M. Telehealth adaptation of the Lidcombe Program of Early Stuttering Intervention: Five case studies. Am J Speech-Lang Pathol 2004; 13(1):82-90.

\section{Objectives}

1. Identify the primary difficulties related to speech/language service delivery in school-based settings

2. Describe a rubric that can be used to compare face-to-face and telepractice service deliveries

3. Appraise a comparison of face-to-face and telepractice service delivery outcomes in elementary-school children

\section{P45 IMPACT OF PEDIATRIC TELEPSYCHIATRY ON PATIENT AND FISCAL OUTCOMES}

\section{PRESENTER:}

John Thomas, PhD, MSSW, BBA, Director of Telehealth, Co-Director Colorado ECHO

University of Colorado, Children's Hospital Colorado, Aurora, CO

Introduction: The management of pediatric behavioral health emergencies is a growing issue. In the past, if pediatric patients presented at a Children's Hospital Colorado network site with a behavioral health issue, they would be transferred to the main campus by ambulance. In the last year, CHCO deployed telemedicine in NOC sites with the purpose of providing telepsychiatry consults resulting in fewer transfers and improved outcomes. Despite an increasing number of consultations, little is known about the impact these telepsychiatric consultations have on clinical and fiscal outcomes.

Methods: Through a retrospective chart review we determined: quality of care (as measured by outcomes (discharge/transfer); patient/provider satisfaction with care delivery; appropriateness of care utilization; and cost effectiveness compared to usual care (telephone consultations) and transfer by ambulance to main ED. For reference (comparison) we compared the practice model of network of care sites continuing to conduct practice as usual (no telemedicine) during the same time period. The telemedicine capacity was deployed to 5 remote emergent care sites between December 2014 and May 2015. The purpose of the effort was to provide the emergency rooms in these network of care sites with access to behavioral health expertise. In this study we included a cohort of patients presenting at the emergency rooms of network of care locations with psychiatric symptoms.

Study Design: The study period for this project will be January 2014August 24, 2015.

Data Collection: Medical records will be copied by remote site personnel and de-identified of all patient and hospital identifications. These deidentified medical records will be used to abstract the necessary data to accomplish the objectives of this proposal. The investigators cannot ascertain the identity of the subjects. The de-identified charts will be scanned and securely uploaded into RedCap. The charts will then be reviewed to obtain data for our outcomes: we will review and score the surveys that are a part of the medical records as to the quality of care experienced by the patient and provider(s) (remote and treating sites); we will determine from the chart the patient disposition; knowing the disposition of care, we will determine the costs based on standardized average transfer, ER admission charges. We are therefore collecting several data points: costs of care, patient disposition and diagnosis.

Data Analysis: Descriptive analyses will be conducted to describe the sample characteristics, mean differences and standard deviations. Bivariate analyses and multivariate analyses will be conducted to report correlations between variables and covariates and any significant predictors of outcome measures. Economic Evaluation: To evaluate the economic efficiency of telepsychiatry, we will apply standard economic evaluation methods to data collected. Knowing the disposition of the patient, we will assign any of the following costs from external data sources: (1) Cost of emergency care treatment and (2) Transportation costs.

Results: A total of 250 patients presented with psychiatric symptoms during the time period. The mean age was 12.6 (SD 3.7 years). 51\% were 


\section{ePOSTER PRESENTATIONS ABSTRACTS}

male, 80\% were Caucasian, 6\% > 1 Race, 4\% Mexican-American, 4\% African-American, 2\% Asian and 4\% other. Diagnoses: Suicidal ideation 62\%, Major Depression 14\%, Anxiety 14\%, Aggression $=3 \%$, Don't know $=7 \%$. For telephone consultation, 92\% of patients were transferred to main ED, compared to the following results for the patients at the sites using telemedicine: 55\% discharged directly home, 15\% direct admit to inpatient (triage done at remote ED and transferred directly), 9\% direct admit to another facilty (compared to previous practice of tranferring first to ED and then transferring back out), 19\% were transferred to main campus emergency room, 2\% technical failure. Satisfaction surveys: 89\% felt comfortable completing their medical visits via technology, 93\% thought visit was easier to complete than an in-person, 82\% would accept "invitation" for other providers to conduct visit by telemedicine, $84 \%$ recommend the technology to others, 93\% thought the visit reduced inconvenience, 91\% thought telemedicine visit reduced time missed from school/work, and $89 \%$ thought telemedicine visits reduced delays for next available appointment.

Conclusions: Tele-enabled psychiatric care in emergency rooms may be an effective care model to increase access to psychiatric triage care, reduce unnecessary costs and trauma of ambulance travel and reduce costs of delivery of care. Patients and parents reported high satisfaction with care being delivered via technology as well as a desire to complete a virtual clinic appointment again and willingness to accept invitations from other speciality providers which may result in increased use of telemedicine integration over time. The convenience and ease of receiving their psychiatric consult remotely resulted in significant time savings for patients and time savings for providers. Further evaluation of this model is ongoing related to the cost benefit analysis and a parameter of use analysis that will be able to determine the $\%$ of use in the system when the cost benefit of using telehealth over in person care will demonstrate itself.

\section{Objectives}

1. Cost benefit of providing traditional mental health services via technology

2. Importance of executive buy-in for effort

3. Improved outcomes comparable to previous practice

\section{P46 DEPLOYING A PEDIATRIC TELEHEALTH PILOT PROGRAM: PROJECT MANAGEMENT APPROACHES, SUCCESSES, AND LESSONS LEARNED \\ PRESENTER: \\ Christopher Shafer, DHA (c), MBA, Director of Telehealth Shriners Hospitals for Children, Tampa, FL}

Shriners Hospitals for Children (SHC) operates 22 hospitals throughout North America, each with large geographic catchment areas requiring many of their patients to travel long distances for care. SHC was presented a challenge to identify and develop a solution that would allay this geographic problem, increase patient access to care, raise the level of convenience to patients and their families, and mitigate the amount of transportation expense incurred by the organization. Shriners Hospitals for Children (SHC) turned to telehealth as the solution to address those challenges. In 2015, the Board of Directors and Trustees of SHC approved a plan to invest, design, and implement a robust telehealth program at three of its hospital sites, with an anticipated system-wide rollout beginning in 2017. The program includes two Tele-Orthopedic sites and one Tele-Burn site in the pilot stage of the project. Telehealth, while not being an entirely new concept to SHC, had no formal structure in place prior. This fragmented approach often led to great frustration on the part of physicians and administrative staff, causing the near abandonment of telehealth utilization altogether. The challenge for the Director of Telehealth, who served as the project manager in the implementation of the Telehealth Pilot program, was taking this large undertaking and breaking it into manageable parts. A clear plan and accountable leadership, combined with engaging a multifarious group of stakeholders and solidifying their buy-in, were crucial components to the success of the project, and ultimately the program. Project management is vitally important in the deployment of any telehealth program, and poor execution can have negative impacts to the program post go-live. Learning from those that have walked down the implementation path prior, can help ensure others have a successful project, and ultimately a successful telehealth program.

SHC Telehealth Case Study Presentation Outline:

1. High-level overview of the SHC organization and why telehealth is such a vital component and complement to the services provided to their pediatric patients

2. Description and objectives of the SHC Telehealth Pilot program

3. Project Management Approaches:

a. Identifying the strategic value of telehealth

i. What are the project objectives?

ii. What does the final state look like?

iii. How can we measure key performance metrics (KPMs)?

b. Importance of project scope

c. Project Governance

d. Project Structure

e. Project Organization

f. Development of project timeline

g. Implementation roadmap

4. Project Successes

5. Lessons Learned

6. Questions and Answers

1. Cuyler R, Holland D. (2012). Implementing telemedicine: Completing projects on target, on time, on budget. Bloomington, IN: Xlibris Corporation.

2. Glandon G, Smaltz D, Slovensky, D. (2008). Information systems for healthcare management ( $7^{\text {th }}$ ed.). Chicago, IL: Health Administration Press.

Objectives

1. Telehealth project management approaches

2. Strategies for success

3. Lessons learned from prior implementation

\section{P47 IMPACTING PEDIATRIC OUTCOMES THROUGH A VIRTUAL COMPREHENSIVE APPROACH}

\section{PRESENTER:}

Elizabeth Cothren, MSN, RN, Assistant Vice President of Telemedicine Ochsner Health System, New Orleans, LA

Pediatric specialty support is a frequently requested telemedicine service among many urban and rural networks. This is typically coupled with poor regional health indicators for children. Several factors contribute to the determents of these poor outcomes, including limited or no access to a variety of maternal and fetal medicine and pediatric specialist. A comprehensive multispecialty approach must be taken to impact and improve infant mortality, postpartum depression, and childhood outcomes in these underserved areas. Traditional pediatric programs will provide access to a pediatric specialist via telemedicine visits in a reactive approach. A consult is requested, provided, and the link to the specialist is ended with little or no follow-up. This case study will highlight the approach one quaternary center took to provide a comprehensive clinical solution to high-risk deliveries and pediatric care using a system of interconnected virtual applications. Our program begins with the assessment and pro-active approach of telemedicine visits for high-risk pregnancies, where a specialist can follow a mother and her baby through delivery. This can be done without a visit to a large medical center and is proven to be cost effective. A high-risk remote monitoring unit was 
developed to support guidance and consultations during complicated deliveries. Partnerships with the pediatrics specialty departments were created to facilitate a smooth transition to appropriate levels of pediatric specialty care. These virtual visits range from scheduled to urgent on demand visits including neonatal specialty visits, live reading of pediatric echo cardiograms, and EEG interpretation for hypothermia management. This program also supports critical care of pediatric patients in emergency departments. Lessons learned in the development and ongoing management of this comprehensive solution will be discussed. Technology challenges and solutions will be highlighted, and quality outcomes including decreased unexpected NICU admits, and improved birth outcomes will be discussed.

\section{Objectives}

1. Identify the need for a multi-speciality approach in Pediatrics

2. Define elements remote monitoring for labor and delivery

3. Define elements of a virtual pediatric speciality program

\section{Track: Operations}

\section{SUNDAY, MAY 15, 2016}

\section{4:45 PM-5:10 PM Sunday, May 15, 2016}

\section{EPOSTERS: MEET THE AUTHOR IN THE ATA EXPERIENCE ZONE/EXHIBIT HALL}

\section{P2 10 YEARS OF PROVIDING SUPPORT - THE NATIONAL TELEHEALTH RESOURCE CENTERS}

\section{PRESENTER:}

Kristine Erps, Administrative Director

Southwest Telehealth Resource Center, Tucson, AZ

The National Telehealth Resource Centers (TRCs) have been providing health care professionals, legislators, federal and other officials, and the public comprehensive, unbiased information related to telehealth for 10 years. The TRCs are non-partisan, unbiased sources of information and assistance who offer their services to anyone interested in pursuing telehealth. There are fourteen TRCs - 12 Regional Centers and 2 National Centers focused on technology assessment and telehealth policy.

The TRCs must have a wide range of expertise and information to meet the demands of their constituencies. The questions range from the most basic such as what is telehealth, to complicated questions that include fine points of policy related to HIPAA, to technical queries on issues related to a specific piece of equipment. To assess the impact of the TRCs, data were collected from the TRCs for the period June 1, 2014 - June 31, 2015. This period was chosen as it is the most recent full year worth of data after all TRCs had been funded and were operating at full capacity (given that they started at different points in time).

The first variable assessed was the number of types of Technical Assistance provided. The results are presented in Table 1 . The most common request (X2 = 456.8, $\mathrm{p}<0.0001)$ made by TRC customers was for Program Development \& Operational Support followed by Reimbursement Provisions, Other and Legal $\&$ Regulatory. Market Analyses, Project Evaluation and Readiness Assessments were the least common types of requests. These data suggest that the majority of the TRC customers are primarily concerned with telemedicine operations rather than early stage investigational aspects associated with determining if there is a need for telemedicine. Best Practices, Strategic Planning, Technology and Equipment issues are the second major group of inquiry types, again suggesting that customers are beyond the early phases of implementing telemedicine and are actually starting up or upgrading programs.
Table 1. Technical Assistance provided by TRCs.

\begin{tabular}{|c|c|c|c|c|c|c|}
\hline & Average & SD & Minimum & Maximum & Median & IQR \\
\hline $\begin{array}{c}\text { Program } \\
\text { Development \& } \\
\text { Operational } \\
\text { Support }\end{array}$ & 107.71 & 146.06 & 2 & 569 & 63.00 & 102.00 \\
\hline $\begin{array}{c}\text { Legal \& } \\
\text { Regulatory } \\
\text { Issues }\end{array}$ & 73.86 & 63.21 & 10 & 214 & 56.00 & 82.00 \\
\hline $\begin{array}{c}\text { Readiness } \\
\text { Assessments }\end{array}$ & 7.07 & 9.94 & 0 & 36 & 2.00 & 11.00 \\
\hline $\begin{array}{c}\text { Business model } \\
\text { development }\end{array}$ & 14.79 & 22.51 & 0 & 72 & 6.50 & 17.00 \\
\hline $\begin{array}{c}\text { Project } \\
\text { Evaluation }\end{array}$ & 8.00 & 10.03 & 0 & 35 & 5.50 & 10.00 \\
\hline $\begin{array}{c}\text { Sharing of Best } \\
\text { Practices }\end{array}$ & 38.57 & 39.19 & 2 & 129 & 28.00 & 34.00 \\
\hline $\begin{array}{c}\text { Strategic } \\
\text { Planning }\end{array}$ & 26.79 & 36.88 & 0 & 130 & 13.50 & 26.00 \\
\hline $\begin{array}{c}\text { Market } \\
\text { Analysis }\end{array}$ & 8.50 & 11.50 & 0 & 34 & 4.50 & 16.00 \\
\hline $\begin{array}{c}\text { Reimbursement } \\
\text { Provisions }\end{array}$ & 99.79 & 143.31 & 5 & 559 & 55.00 & 120.00 \\
\hline $\begin{array}{c}\text { Technology } \\
\text { Assessment }\end{array}$ & 24.36 & 33.32 & 0 & 124 & 17.50 & 20.00 \\
\hline $\begin{array}{c}\text { Equipment } \\
\text { Selection }\end{array}$ & 29.50 & 32.61 & 1 & 120 & 17.00 & 37.00 \\
\hline $\begin{array}{c}\text { Tools and } \\
\text { Templates }\end{array}$ & 23.93 & 17.39 & 2 & 66 & 19.50 & 23.00 \\
\hline \begin{tabular}{c} 
Other \\
\hline
\end{tabular} & 123.71 & 17.39 & 0 & 428 & 86.00 & 156.00 \\
\hline
\end{tabular}

Table 2 shows the means by which TRCs are contacted. The most common means $(\mathrm{X} 2=1054, \mathrm{p}<0.0001)$ is meeting people at conferences followed by contact via websites and in person contacts. Email and personal contacts are common as well, with the 800 numbers being the least common mode.

Table 2. Means by which the TRCs are contacted for Technical Assistance.

\begin{tabular}{|c|c|c|c|c|c|c|}
\hline & Average & SD & Minimum & Maximum & Median & IQR \\
\hline In Person & 525.57 & 929.01 & 11 & 2764 & 90.50 & 285.00 \\
\hline Conference & 853.36 & 2998.35 & 0 & 11266 & 19.00 & 72.00 \\
\hline $\begin{array}{c}\text { Personal } \\
\text { Contact }\end{array}$ & 181.14 & 581.84 & 0 & 2200 & 13.50 & 57.00 \\
\hline Web site & 791.29 & 1974.15 & 0 & 5986 & 28.50 & 33.00 \\
\hline $\begin{array}{c}800 \\
\text { number }\end{array}$ & 99.93 & 192.11 & 0 & 742 & 37.50 & 49.00 \\
\hline Email & 340.57 & 597.91 & 36 & 2136 & 10.00 & 130.00 \\
\hline
\end{tabular}

Finally we assessed different ways in which the TRCs provide outreach, advertise their offerings and the benefits of telemedicine (Table 3). As reflected above, attending conferences and making presentations to large audiences is the most common type $(\mathrm{X} 2=7020, \mathrm{p}<0.0001)$ of outreach. Peer-to-peer connections are common as well, while hosting conferences and writing articles are less common.

Table 3. Types of outreach performed by the TRCs.

\begin{tabular}{|c|c|c|c|c|c|c|}
\hline & Average & SD & Minimum & Maximum & Median & IQR \\
\hline Presentations & 27.93 & 32.47 & 0 & 132 & 24.50 & 21.00 \\
\hline Audience & 1722.43 & 2910.19 & 0 & 11266 & 771.50 & 1519.00 \\
\hline $\begin{array}{c}\text { Conferences } \\
\text { Aftended }\end{array}$ & 20.00 & 13.92 & 6 & 51 & 14.00 & 20.00 \\
\hline $\begin{array}{c}\text { Conferences } \\
\text { Hosted }\end{array}$ & 6.29 & 10.10 & 0 & 33 & 1.50 & 3.00 \\
\hline $\begin{array}{c}\text { Peer-peer } \\
\text { Connections }\end{array}$ & 190.29 & 269.10 & 3 & 796 & 51.00 & 327.00 \\
\hline Articles & 4.64 & 5.68 & 0 & 16 & 2.50 & 7.00 \\
\hline
\end{tabular}

The demands for the level of technical assistance the TRCs provide continue to grow, and it is expected that these numbers will continue to rise. As telehealth is increasingly turned to as a solution for expanding services, meeting health needs and utilizing resources more efficiently, the work of the TRCs becomes even more important. As agnostic organizations dedicated to providing accurate, unbiased information and resources not influenced by any industry or political organization, the TRCs play a major role in the proliferation of telehealth delivered health services, especially for those in underserved areas. Additionally, the uniqueness of the TRCs, regionalized specialization but working collaboratively together to 


\section{EPOSTER PRESENTATIONS ABSTRACTS}

provide a consistent, cohesive voice, positions them perfectly to deliver accurate, comprehensive information to the wide range of parties interested in telehealth.

\section{Objectives}

1. Identify the key types of technical assistance provided by the TRCs to better understand what the telemedicine community still needs help with.

2. Provide attendees with information about where the TRCs are, how to contact them, and what services they provide.

3. Describe the types of outreach provided, the best ways to contact the TRCs, and how to obtain their services.

\section{P3 KNOWLEDGE AND USAGE OF MOBILE HEALTH APPLICATIONS AMONG RESIDENT DOCTORS IN OGUN STATE, NIGERIA}

\section{PRESENTER:}

Akintunde Akinpelu, BSc, MBBS Student

Olabisi Onabanjo University, Sagamu, Nigeria

Introduction: Nigeria is the most populous black nation in the world and has the highest penetration of mobile devices among Africans, health care providers included. With the proliferation and popularity of mobile devices such as tablets, smartphones, and other personal devices among healthcare providers, also comes an exponential increase in healthcare applications. Over 100,000 healthcare applications are known today. These applications cover a broad spectrum of services for medical training, disease diagnosis, treatment calculations, literature searches, clinical communication, health information systems, and general patient care. Some applications may have multiple or overlapping functions. Other applications are used as accessories to medical devices, and some transform the mobile platform into a medical device. Smartphone and tablet use among healthcare professionals has become widespread and will continue to grow in the coming years. The potential benefits that come with them can only be realized when the healthcare providers are aware of these applications and are committed to using them. The purpose of this study was twofold: to evaluate the knowledge and usage levels of medical applications among resident doctors and to determine the barriers to the adoption and usage of these applications. In May 2015, a survey was distributed to 120 resident doctors at the five residency training centres in Ogun State, Southwest Nigeria; asking them about their usage of mobile devices, and adoption of health apps on these devices for work purposes.

Results: There is a total of 120 respondents with response rate of $45.98 \%$ of which $81.7 \%$ are male resident doctors and $18.3 \%$ female. Doctors in residency training and medical officers in Psychiatry, Obstetrics and Gynaecology, Internal medicine, Family medicine, Community medicine, Surgery, Paediatrics, Paediatrics surgery and Opthalmology were involved. The mean age of the respondents is $35.13 \pm 4.93$, the average years spent in training is $3.79 \pm 1.89$ while the average years of experience is $8.17 \pm 3.75$. The study shows that all respondents (100\%) use smart phones of which $98.3 \%$ of them use internet enabled smart phones and 78.3\% are Android operating system. The challenges faced in the usage of mobile health applications among the resident doctors are erratic internet connectivity (78.3\%), excessive data consumption (35.8\%), high prices of smart phones and tablets (24.2\%), not easy to use mobile apps (9.2\%) and unavailability of relevant health apps in apps markets (26.7\%). The research shows that respondents are mostly aware of apps that can be used for making video calls/conferencing (93.3\%) while the most frequently used application is med apps for education (47.5\%). This study shows that 23.3\% have poor knowledge, $27.5 \%$ have average knowledge and $49.2 \%$ have good knowledge about mobile health applications. The study also shows that $23.3 \%$ are average users of mobile health applications while majority $(76.7 \%)$ are poor users. There was significant association between area of residency training and awareness of medical application on mobile platforms using chi square test of association at 95\% confidence interval $[\mathrm{X} 2(8)=31.51, \mathrm{p}=0.001]$. The study also found out that years of training $(<6$ or $\geq 6$ years) as resident has significant association with knowledge of mobile health application using chi square at 95\% confidence interval $[\mathrm{X} 2(2)=14.59$, $\mathrm{p}=0.001]$.

Conclusion: Smart phones are used by majority of resident doctors and level of awareness among them is very high. However, there is a need for specific training on how to integrate mobile medical apps into their work patterns.

1. Mosa AS, Yoo I, Sheets L. A Systematic Review of Healthcare Applications for Smartphones. BMC Med Inform Decis Mak 2012; 12:67.

2. McAlearney AS, Schweikhart SB, Medow MA. Doctors' experience with handheld computers in clinical practice: qualitative study. BMJ 2004; 328(7449):1162.

3. Lee VC. Mobile Devices and Apps for Health Care Professionals: Uses and Benefits. P T 2014; 39(5):356-64.

\section{Objectives}

1. To evaluate the knowledge of mobile health applications among resident doctors in Ogun state, Nigeria

2. To recognize the perceived barriers to the adoption and usage of mobile medical apps among resident doctors in Ogun State, Nigeria

3. To assess the usage of mobile health applications among resident doctors in Ogun state, Nigeria

\section{P4 PERSPECTIVES OF DEVELOPMENT OF TELEMEDICINE IN POLAND}

\section{PRESENTER:}

Paweł Januszewicz, Professor Doctor of Medical Sciences, Director of Institute of Nursing and Health Sciences

University of Rzeszów, Rzeszow, Poland

Introduction: There are no clear legal regulations defining telemedicine and legalizing its ability to function and that is the most important issue in the aspect of organizing activities in this area in Poland [1-3]. Legislative barriers are reinforced by the relatively low awareness of its benefits among politicians, healthcare professionals, patients and the rest of the population $[4,5]$. This contributes to a very rare use of new technological solutions allowing for the exchange of medical information through telecommunication tools to improve patient's health.

Aim: The aim of this study was to evaluate the possibilities of use of applications supporting health and a healthy lifestyle among employees of companies owning or intending to purchase mobile devices and, at parallel, assess their health behaviors.

Materials and Methods: The study group was 1942 people, including $70.1 \%$ of women and 29.9\% of men. The age division of the group ranged between: 18 29 years (32.6\%); 30-49 years (45.6\%); 50-64 years (19.8\%); > 65 years $(2.0 \%)$. As for the social characteristics of the group, 52.8\% lived in a city and $47.2 \%$ lived in a village. The education of particular groups was as follows: $8.0 \%$ vocational; 25\%-high school; 17.3\%-BA/BSc; 49.8\%-MA/MSc. The research was carried out by diagnostic survey questionnaire technique using two versions of the questionnaire interview. The study was conducted in the period from June 2014 to January 2015 among 1,942 people, including 734 employees of the University of Rzeszów, 490 employees of the Aviation Valley and 718 patients using primary health care services. The study used a questionnaire and Health Behaviors Inventory by J.Juczyński. The study was carried out with the use of statistical method STATISTICA 11.0. The study was financed from the funds allocated to research statutory (507) funding university research projects in accordance with the regulation No.100/2014 of Rector of the University of Rzeszów. The Bioethics Committee of the Regional Medical Chamber in Rzeszów approved the study, which was conducted in accordance with the Declaration of Helsinki. Results: Current state of the use of mobile healthcare devices and the perspective of this use in future. Only 7\% out of the research group declared the use of health applications currently, while 75\% of the population researched declared their interest in such use. At the same time, $70 \%$ of the group declared their interest in the use of healthy lifestyle applications. The current use and 


\section{ePOSTER PRESENTATIONS ABSTRACTS}

potential increase in the number of people using mobile health applications based on sex, place of residence, age and education: Despite the fact that men currently use the health applications more often than women (8\% men vs 6\% women), it is women who declare more interest in the use of healthy lifestyle applications (71\% women vs $66 \%$ in men), and in health and sickness applications (76\% women vs $71 \%$ men). As for age, it is mostly young people, between the age of 18 - 29 who use the health and healthy lifestyle applications $(9 \%$ currently use such applications, while almost $80 \%$ declare their interest in such applications. As for the place of residence of respondents, there are no clear differences between both current and declared use of mobile applications. As for the education level, it is mostly respondents with higher education (BA and MA) who are at the highest level using and also declaring their willingness of use of mobile health applications. Level of the health behaviors among the respondents is: low $42 \%$, medium 35\%, high $23 \%$. Research show that the level of health behaviors is growing together with the interest in the mobile healthcare applications.

Conclusions: (1) The respondents present great potential for growth opportunities in the future in the number of people using mobile health applications; (2) The highest percentage of respondents exhibited a low level of health behavior; and (3) As the level of health behaviors in the study group is growing, also their interest in mobile applications is growing.

\section{Resources:}

1. Zwiefka A. Aspekty prawne i regulacje jako bariery wdrażania innowacji telemedycznych. http://www.kigmed.eu/pliki/konferencja_2012-11-13/ Antoni_Zwiefka_-_UMWD.pdf, www.intermed-c2c.eu

2. Najbuk P. Telemedycyna aktualny stan prawny i perspektywy zmian $\mathrm{w}$ przyszłości. http://blog.dzp.pl/pharma/telemedycyna-aktualny-stanprawny-i-perspektywy-zmian-w-przyszlosci/

3. Ustawa o zawodach lekarza i lekarza dentysty. http://isap.sejm.gov.pl/ DetailsServlet?id=WDU19970280152

4. Bujok J, Gierek R, Olszanowski R, Skrzypek M. Uwarunkowania rozwoju telemedycyny w Polsce. Potrzeby, bariery, korzyści.

5. analiza rynku, rekomendacje. http://medgo.pl/wp-content/uploads/2014/ 08/Raport-telemedycyna-fin-22.07.2014.pdf

6. Telemedycyna rozwój i bariery. http://www.eioba.pl/a/4chh/telemedy cyna-rozwoj-i-bariery

7. http://www.zwodnik.pl/kolekcja/android/darmowe-aplikacje-zdrowotneoraz-fitness-na-androida-2/

Keywords: Mobile healthcare applications, health behaviors, potential of use

\section{Objectives}

1. Assess potential of telemedicine in Poland

2. Find out about the connection between health behaviors and the use of mobile phone health applications

3. Find out about the population's interest in health applications use

\section{P5 WHAT IS THE EVIDENCE FOR THE ECONOMIC VALUE OF DIFFERENT LEVELS OF VIRTUAL HEALTHCARE? A SYSTEMATIC REVIEW}

\section{PRESENTER:}

Leslie Wilson, PhD, Professor

University of California, San Francisco, San Francisco, CA

Introduction: The United States (U.S.) healthcare system continues to face increasing costs, with year-over-year projected cost increases that now exceeds the rate of increase of the Gross National Product (GNP). Virtual healthcare has been expanding rapidly in the past five years. Its integration within the U.S. healthcare system is driven primarily by its perceived ability to increase access in a cost-efficient way. However, there is little economic research that addresses the large diversity of virtual healthcare products and initiatives and how they are being integrated into the US healthcare landscape. The goal of this study is to evaluate the published economic evidence for virtual healthcare, analyze how well it supports the growth of the current virtual healthcare environment and suggest what evidence is needed.

Methods: A systematic literature search was conducted through PubMed and Cochrane Library databases from 2010 to 2015 using a search algorithm utilizing Mesh terms for economics and types of virtual healthcare. The initial 802 results were filtered by English-language, humans, and an abstract. The resulting 230 abstracts were read for relevance to inclusion and exclusion criteria; an economic outcome. We excluded studies that were telephoneonly, only web-based information dissemination, and foreign settings not applicable to US healthcare. 25 abstracts remained, including 5 systematic reviews, 4 reviews, 3 randomized controlled trials, 11 cohort studies, and 2 case series.

Results: Many different types of economic evaluations were represented in our review, from simple discussions about cost to comprehensive costeffectiveness decision trees and Markov models. The "gold standard" economic study is one that measures both costs and outcomes such as a cost-effectiveness, cost-utility, or cost-benefit study (Gold, 1996). Other economic studies examined just costs or were cost-consequence analyses, where the outcome is unknown but varied to determine at what level of outcomes a comparison is cost-effective. Economic studies also varied by if they were modeled or part of a clinical trial or other prospective study design. Modeled studies were either Markov models which allow for transition probabilities that can change over time, or simple decision analytic models. Costs were measured either directly or estimated from the literature. Some cost estimates were based on little real evidence except logic or speculation. Economic studies also varied by their perspective, with a societal perspective being the "gold standard" because they include all costs and outcomes to society. Others took either a healthcare system, industry, or other entity perspective and then measured only costs relevant to that system. The studies in our review covered all of these study types, but there were few studies of the highest quality.

Patient Access and Convenience: We grouped studies by their relevance to patient impact in today's healthcare system. For example, several studies addressed the ability of telemedicine to improve patient access and convenience. These included studies that only compared a few items of costs so that no accurate economic value conclusion could be drawn. There were two relatively strong cost-effectiveness studies using quality-adjusted life years (QALYs) which demonstrated the cost-effectiveness of two different virtual healthcare programs designed to improve patient access to cervical screening and to mental health. Preventive Care and Continuity: Our second grouping of economic studies addressed the ability of virtual healthcare to provide preventive care and continuity of care. They looked at the use of hub-and-spoke tele-health initiatives and networks and iPhonebased prevention measures in various chronic diseases and disorders. Most of these studies were simple and often incomplete cost studies, but there was a strong cost-effectiveness study demonstrating that asynchronous home-based blood pressure monitoring could cost-effectively control hypertension. There were also several economic evaluations that addressed tools for monitoring diabetes control and tele-retinal screening programs that had fairly good evidence of economic value.

Conclusions: The current level of evidence is promising, but clearly not comprehensive; focusing primarily on the value of telemedicine programs rather than more complete "stand-alone" virtual healthcare systems or virtual programs integrated into existing healthcare systems. The current landscape is much broader and less tailored to traditional telemedicine initiatives such as tele-stroke, tele-ICUs, and remote blood pressure monitoring. This discrepancy between the literature and what currently exists may be a reflection of how quickly the virtual healthcare landscape is evolving, leading to a lag in the body of strong economic evidence to support adoption of current initiatives. We suggest more rigor in the design of economic studies. In addition, we 
suggest the current virtual healthcare technologies be identified for analysis in different patient groups and within different healthcare systems. These systems should evaluate their ability to provide cost-effective care using rigorous economic analytic designs that include patient preference. Much work needs to be done to generate relevant economic evidence to better understand the cost-effectiveness of the current virtual healthcare landscape.

1. Kim A, Lai J, Lamont E, Szeto D, Wilson L. Virtual health care evaluated by UCSF and Stanford. Retrieved from http://pharmacy.ucsf.edu/ news/2015/08/virtual-health-care-evaluated-ucsf-stanford (Accessed Aug 7, 2015)

2. Urguhart AC, Antoniotti NM, Berg RL. Telemedicine - an efficient and cost-effective approach in parathyroid surgery. Laryngoscope 2011; 121(7):1422-425.

3. Anderson ER, Smith B, Ido M, Frankel M. Remote assessment of stroke using the iPhone 4. J Stroke Cerebrovasc Dis 2013; 22(4):340-44.

4. Gold MR, Siegel JE, Russell LB, Weinstein MC. (1996). Cost-effectiveness in Health and Medicine. Oxford, England: Oxford University Press.

\section{Objectives}

1. Summarize the economic evidence for virtual healthcare in the United States

2. Assess the applicability of the economic evidence's to the current United State virtual healthcare landscape

3. Identify the types of economic and patient preference evidence needed to meet the growing virtual healthcare integration

P6 BUILD A STRONG TELEHEALTH FOUNDATION FOR SUCCESSFUL POPULATION HEALTH MANAGEMENT

\section{PRESENTER:}

Blix M. Rice, MBA, Administrator

Integrated Health Services, Greenville Health System, Greenville, SC

When building a telehealth program, an organization can be tempted to begin with available technology and look for ways to use each potential solution. Excitement builds as innovators, vendors and clinicians engage one another. Financial return on investment becomes difficult to measure in a push to get programs started. Organizations often find clinical departments and administration in conflict around the merits of continued development and optimal prioritization of resource deployment. Greenville Health System is a large integrated delivery system that is taking a different approach to telehealth development. By focusing first on evidence-based clinical workflows, this system is letting form follow function and building a structure in which telehealth emerges from the clinical need rather than creating new ones. GHS has focused its investment on constructing an underlying infrastructure of standardization and technological capability that will permit clinical applications to emerge efficiently to meet the needs of clinical providers. In this session, we will explore potential pitfalls in telehealth program development, consider methods to begin with a population health-focused care model and let telehealth applications fulfill an identified need, rather than the other way around. We will also examine value-based reimbursement models and their potential to accelerate telehealth adoption by making the return on investment more apparent and measurable.

\section{Objectives}

1. Identify the need to connect care model development to telehealth solutions

2. Understand the role value-based reimbursement models play in the development of telehealth programs

3. Apply demonstrated methods to evaluate and modify telehealth development pathways in their organizations

\section{SUNDAY, MAY 15, 2016}

5:10 PM-5:35 PM

Sunday, May 15, 2016

\section{EPOSTERS: MEET THE AUTHORS IN THE ATA EXPERIENCE ZONE/EXHIBIT HALL}

\section{P7 EXPERIENCE REPORT OF SUPPORT TO THE TELEHEALTH CLUSTER OF THE STATE OF PIAUI BY THE SCHOOL OF MEDICINE FOR THE FEDERAL UNIVERSITY OF MINAS GERAIS - BRAZIL}

\section{PRESENTER:}

Humberto J. Alves, MD, PhD, Vice Dean School of Medicine

Federal University of Minas Gerais, Belo Horizonte, Brazil

Introduction: This study aims to present the report of the support by Telehealth Cluster of the School of Medicine of the Federal University of Minas Gerais (NUTEL-FM/UFMG, in Portuguese) for the implementation of the Intermunicipal Telehealth Clusters of the Piaui State, Brazil. The NUTEL began its activities in 2007 with the support of the Ministry of Health through the Secretariat of Work and Education Management (SEGETES) responsible for formulating guiding public policy management, training and qualification of health workers in Brazil. NUTEL is a Technical Scientific Telehealth Center with tradition in the deployment of telehealth resources implementation methodologies and is able to replicate its experience gained by studies and practices. The National Telehealth Program was established in 2007 and expanded in 2011 as Telehealth Brazil Network Program, restructuring the program by adding 63 new institutions including the Piaui State Cluster (PI), namely Carnaubais (municipality of Campo Maior), Floriano (municipality of Floriano) and Cocais (municipality of Piripiri). In 2012, the Ministry of Health Department of Primary Care (DAB) has requested the monitoring of Piaui Clusters by NUTEL in order to coordinate and support activities related to planning and implementation of telehealth resources for expanding the scope of activities offered by health family teams, changing care practices and organization of work process, through the provision of Teleconsultations, web conferencing and Formative Second Opinion. The purpose of the project was to guide the implementation and training steps of local telehealth coordinators on the design, development and management of telehealth resources.

Methods: This is a descriptive study based on information of the main activities carried out by three Inter-municipal Clusters of Piaui State during the implementation process of telehealth points of the Telehealth Brazil Networks Program. The actions consisted of the participation of strategic levels personnel, with continuous monitoring, in the implementation process of organizing stages of activities planning, training of local coordinators, and Program presentation to municipal and state management authorities. The main actions employed consisted of meetings with state management personnel and local coordination, and a representative of Ministry of Health in Piaui to present details of the Support Project; Communication with the clusters via webconferences for construction and application of the implementation process of the telehealth points; Technical visits to the towns to present every step of telehealth management and importance of its tools; Assistance in the IT area for setting up the network infrastructure; Training of Family Health Teams (ESF) for use of telehealth services.

Results: Presented in two phases.

Phase One: Meetings with the strategic levels state management authorities and telehealth coordinators; Videoconferences for guidance on the work process of the clusters and specifications for equipment acquisition for the 270 proposed locations; Technical visit to each municipality for raising awareness of managers and health professionals. 


\section{ePOSTER PRESENTATIONS ABSTRACTS}

Phase Two: Trainings for access to the platform; 169 professionals from 75 participating municipalities trained, and registered to use the telehealth platform for instrument; during the implementation period only, August September/2013, 199 requests for asynchronous teleconsultation took place; from the beginning of the process until June 2015 it totaled 4,688 teleconsultations.

Discussion: The NUTEL-FM/UFMG proposed to contribute in this project, with the following dimensions: structuring and implementation of the training course: "Structuring telehealth projects: main aspects involved," directed to managers and cluster coordinators. The participants in the course had insufficient telehealth management knowledge. This deficiency resulted in a positive move for the initial acquisition of these skills. During Phase Two, the first training cycle for Family Health Team (FHT) also took place, with the participation of the coordinators. The following was recommended: greater proximity between telehealth cluster coordinators and FHT personnel, and better ICT infrastructure for all telehealth points.

Conclusion: The process of supporting the cluster was instrumental in implementing the actions of Telehealth Brazil Networks Program in the Piaui State. It can be inferred that there is still a gap as it relates telehealth resource management and technological knowledge on the part of clusters. The poor infrastructure is still an impeding barrier for activities and implementation of telehealth resources by management. Considering the support proposed, one can conclude that the project is successful so far. One can evaluate that the method used for the deployment needs better consistency of the implementation strategies of telehealth resources in order to encourage improvement initiatives of services offered.

1. Brasil. Ministério da Saúde. Manual de Telessaúde para Atenção Básica / Atenção Primária à Saúde / Ministério da Saúde, Universidade Federal do Rio Grande do Sul. - Brasília : Ministério da Saúde, 2012. 123 p. : il. - (Série A. Normas e Manuais Técnicos).

2. Brasil. Ministério da Saúde. Telessaúde Brasil Redes. Brasil, 2010a. Disponivel em: Access: Sept. 12, 2015.

3. Brasil. Ministério da Saúde. Gabinete do Ministro. Portaria no 2.554, de 31 deoutubro de 2011. Institui, no Programa de Requalificação de Unidades Básicas de Saúde, o Componente de Informatização e Telessaúde Brasil Redes na Atenção Básica, integrado ao Programa Nacional Telessaúde Brasil Redes. Diário Oficial da União, Brasília, DF, 2011b, Sec. 1, p. 28-9.

4. Brasil. Ministério da Saúde. Gabinete do Ministro. Portaria no 2.546, de 27 de outubro de 2011. Redefine e amplia o Programa Telessaúde Brasil, que passa a ser denominado Programa Nacional Telessaúde Brasil Redes (Telessaúde Brasil Redes). Brasília, 2011c. Available at: Access: Sept. 12, 2015.

\section{Objectives}

1. Compare implementation experience

2. Apply to their own projects

3. Discuss particular difficulties

\section{P8 BH-TELEHEALTH PROGRAM: ANALYSIS OF TELECONSULTATIONS \\ PRESENTER}

Maria do Carmo B. Melo, Ph.D., Medical Doctor, Professor, Subcoordinator of Telehealth Center

Medicine School, Universidade Federal de Minas Gerais, Belo Horizonte, Brazil

Introduction: The BH-Telehealth Program (Belo Horizonte Telessaúde, in Portuguese) was established in 2004. The program offers support care for the public health system professionals in order to improve access to specialists, reduce cost, and improve the quality of care. Until now, the use of teleconsultation did not achieve its main goal of increasing the resolution of the
Primary Health Care service in the way it was expected. It is important to analyze the perception of managers and teleconsultants specialists, because they are strategic actors in the process and it was not investigated in previous studies.

Methods: The present study analyzed the organizational, technological and cultural factors related to the incorporation of technology through qualitative methodology. Interviews were performed and analyzed according to the content analysis technique. Software was used to analyze the interviews. Quantitative and data collection and analysis were conducted.

Results and Discussions: From 2006 to 2013, 1,827 subspecialist teleconsultations were performed. The data demonstrated that the more requested subspecialists were angiologists $(n=510)$, cardiologists $(n=199)$, endocrinologists $(n=153)$ and neurologists $(n=99)$. Until now, 23 interviews were conducted. The health professionals of the primary care system preferred to request asynchronous instead of synchronous teleconsultation, because they considered it difficult to prepare the case and access the system, moreover they did not have time to do this. The answers indicated that more support from the managers of the services and from the central organization is needed. The teleconsultants required a dedicated time to answer the requests and to respond to the primary care professionals in order to improve the relationship Most referrals can avoid an in-person visit with the specialist. Using a software program, it is possible to identify some categories of problems involving organizational, cultural and personal questions. Behavior change is a challenging process for anyone. The decision of physicians to adopt a new technology such as telehealth can be challenged by their relatively low computer literacy, the possible alteration of their traditional routines, and their high professional autonomy. Possibly that context influenced the technology adhesion.

Conclusion: It is necessary to reflect on the results in order to improve the use of the teleconsultations in the BH-telehealth program. The end result may point to possible strategies for increased use of teleconsultation in $\mathrm{BH}-$ Telehealth program, impacting on user assistance quality, providing a greater link between patients and healthcare team and reducing waiting list for specialist consultation. This system also has the potential to reduce costs by reducing transportation and optimizing patients' daily routine, but the program organization must be reviewed.

\section{Objectives}

1. Describe quantitatively the data of the BH-Telehealth Program

2. Evaluate the perception of managers and teleconsultants specialists about the numbers of teleconsultations request

3. Analyze the organizational, technological and cultural factors related to the incorporation of technology through qualitative methodology

\section{P9 TELEMEDICINE PROJECT - A SOLUTION TO SUPPORT OFFSHORE OIL PLATFORMS \\ PRESENTER: \\ Milton Steinman, MD, Coordinator \\ Hospital Israelita Albert Einstein, Sao Paulo, Brazil}

Introduction: For years, oil and gas companies (OG) have staffed the rigs and platforms with a paramedic, emergency medical technician (EMT), or nurse who would assess the worker and telephone findings to a physician on shore. In these locations, timely access to quality healthcare is becoming more logistically challenging and expensive making it an ideal environment for establishment of eHealth initiatives.

Purpose: To describe the telemedicine project implemented in two OG offshore platforms located at Santos shore, in Brazil.

Methods: Hospital Albert Einstein (HIAE), in Sao Paulo developed a real time telemedicine (TM) offshore program providing 24/7 hours/days of an emergency department (ED) coverage. Platforms were equipped with internet and satellite connections and with telemedicine cart with a video 


\section{ePOSTER PRESENTATIONS ABSTRACTS}

conferencing system outfitted with medical devices and scopes (FES GlobalMed $\circledast$ ). Using two-way video, the doctor conducts the visit in a real-time conversation with the worker and the EMT. Emergency medicine physicians on shore can examine the patient and order electrocardiograms (ECGs) and laboratory tests, allowing them to more accurately assess the patient and determine a plan of care. An electronic medical record keeps all the health information safe, organized and accessible over any distance, and eliminates the problems and delays of a paper-based record system. Strict controls ensure authorized access to the electronic medical record and keep all patient information secure. A helicopter evacuation plan was also implemented based upon standard protocols, divided in routine and emergency plans.

Results: Starting October 2014, a total of 603 offshore workers were treated by doctors over the video telemedicine link during the first twelve months of the service. TM consultation was effective in conducting 554 (91.9\%) cases. Thirty-nine workers were evacuated on a routine protocol and 10 workers ( $1.7 \%$ of the total) under emergency protocol. High audio and video quality connection was established in 515 (86.56\%) calls. The main telemedicine resources used were auscultation, dermatoscopy and oropharyngeal visualization. None of the workers died offshore during the period studied.

Conclusion: In order to improve the quality of medical decisions and the safety of the workers offshore, a telemedicine platform is feasible and offers better and faster decision-making. It also allows reevaluation that improves accuracy, even towards evacuation decisions.

\section{Objectives}

1. Understand offshore telemedicine operation

2. Discuss offshore decision making

3. Discuss offshore evacuation plans

\section{P10 NEEDS ASSESSMENT FOR TELEMEDICINE IN AFGHANISTAN \\ PRESENTER:}

Stephen Fischer, MD, MPH, Preventive Medicine Physician

Public Health Department, Walter Reed National Military Medical Center, Bethesda, MD

Mitigating the immense global, national, and personal burden of the lack of access to healthcare for millions of underserved Afghans will require a greater emphasis on coordination between local and national actors through improved communication infrastructure and access to Health IT. Afghanistan has been plagued with chronically poor health, metrics that have only begun to improve in recent years, following massive support from foreign donors. This study investigates telemedicine in Afghanistan and its ability to form a critical component of best practices in the effective health system strengthening the country. A comprehensive literature review and survey of Afghanistan's key public health leaders and stakeholders was conducted to investigate the implementation, management and sustainability of telemedicine initiatives. The results indicated the lack in technical capacity / resources, funding, policies / procedures to support telemedicine efforts and the need for greater demand and operational stability of such programs. Recommendations were then made in the aim to help define a national Afghan telemedicine plan, establish national standards and operational aspects, and better identify and evaluate all stakeholders needed for a successfully sustainable global telemedicine network for resource-poor, austere environments.

1. AAnensan, D. (2013) "Spatialepidemiology.net | Mapping Infectious Disease Epidemiology."Spatialepidemiology.net | Mapping Infectious Disease Epidemiology. Imperial College London, n.d. Web. 01 June 2013.

2. American Telemedicine Association. 1 May 2013 (http://www.atmeda .org/news/overview.htm).
3. California Telemedicine and eHealth Center, A Glossary of Telemedicine and eHealth (Sacramento, CA: California Telemedicine and eHealth Center, 2006) 25.

4. Durrani H, Khoja S, Naseem A, Scott RE, Gul A, Jan R. Health needs and eHealth readiness assessment of health care organizations in Kabul and Bamyan, Afghanistan. East Mediterr Health Journal, 2012;18(6): 663-70.

5. Harcke T, Montilla J, Statler J. Radiology in a hostile environment: experience in Afghanistan. Military Medicine 2006; 171(3):194-99.

6. Khoja, K. (2009). Connecting a Nation: Roshan Brings Communications Services to Afghanistan. Innovations: Technology, Governance, Globalization 2009; 4(1):33-50.

7. Khoja S, Scott R, Casebeer A, Mohsin M, Ishaq A, Gilani S. e-Health Readiness Assessment Tools for Healthcare Institutions in Developing Countries. Telemed J E Health 2007; 13(4):67-84.

8. Ministry of Public Health. Afghanistan Health Survey 2006: Estimates of Priority Health Indicators for Rural Afghanistan, MOPH, Kabul (2006), p. xi.

9. Nieburg P. Improving maternal mortality and other aspects of women's health: the United States' global perspective. Center for Strategic \& International Studies Journal 2012; 23(4):23-31.

10. "Roshan Announces launch of Afghanistan's first telemedicine project." Multimedia Production. Strategic Distribution. Roshan, Aga Khan Development Network, 20 June 2007. Web. 26 May 2013.

11. Patterson V, Swinfen P, Swinfen R, Azzo E, Taha H, Wootton R. Supporting hospital doctors in the Middle East by email telemedicine: something the industrialized world can do to help. J Med Internet Res 2007; 9(4):e30.

12. Sanghvi H, Ansari N, Prata N, Gibson H, Ehsan A, Smith J. Prevention of postpartum hemorrhage at home birth in Afghanistan. Int $J$ Gynaecol Obstet 2010; 108(3):276-81.

13. UCSD Telemedicine Statistics and Data. (2012). Retrieved November 17, 2012, fromhttp://telemedicine.ucsd.edu/

14. World Health Organization. Causes of Death Statistics. (2002, October 9). Retrieved November 19, 2012, from who.org: www.who.org

\section{Objectives}

1. Understand the current state of telemedicine in Afghanistan, public and private initiatives

2. Afghanistan Ministry of Public Health eHealth policy framework

3. Afghanistan population health metrics, challenges and opportunities

\section{P11 TELEMEDICINE IMPLEMENTATION CHALLENGES BY HEALTHCARE PRACTITIONERS}

\section{PRESENTER:}

Olatunji Obikunle, Doctor of Business Administration, Student

Walden University, Minneapolis, MN

As a result of the numerous challenges in healthcare accessibility faced by the people who live in rural and remote areas of the US, the author looks at the various challenges faced by healthcare practitioners when adopting telemedicine. The purpose of this qualitative study was to examine these challenges and come up with the most dominant pitfalls experienced by these healthcare practitioners and how new entrants coming to the emerging field can avoid those problems. A standardized conceptual framework was used as the basis of the investigation. Physicians were interviewed in a face to face manner based on a set of questionnaires. Themes were identified through the use of industry acceptable software, and appropriate solutions that would assist other healthcare practitioners were advanced. The issue of cost implications and benefits were evaluated. Implications for social change were enumerated, and recommendations that would benefit rural inhabitants, healthcare practitioners, and various stakeholders were provided. 
1. Fanale CV, Demaerschalk BM. Telestroke network business model strategies. J Stroke Cerebrovasc Dis 2012; 21(7):530-34.

2. Faust 0 , Shetty R, Sree S, Acharya S, Acharya U R, Ng E, et al. Towards the systematic development of medical networking technology. J Med Syst 2011; 35:1431-445.

3. Feldstein PJ. (2012). Health care economics. Clifton Park, NY: Delmar/ Cengage Learning.

\section{Objectives}

1. Learn how to adopt telemedicine in hospitals and clinical settings

2. Prepare for the challenges ahead before venturing into the field of telemedicine

3. Optimization processes in successful telemedicine adoption

\section{P12 LEVERAGING MEDICAL RESIDENCY EDUCATION UTILIZING PROJECT ECHO TECHNOLOGY}

\section{PRESENTER:}

Gregory J. Keough, MPH, Program Coordinator

University of Utah, Salt Lake City, UT

Introduction: Graduate Medical Education (GME) for medical residents is an integral part of our healthcare delivery system. GME builds upon the foundation of skills and experiences that individuals gain in medical school, and bring to their clinical practices. Understanding what tools are effective in sharing knowledge and clinical skills in this area can directly, and positively impact patient care. GME utilizing case based-learning has long been established as an effective method of gaining clinical skills [1] and Project ECHO (Extension for Community Health Outcomes) has worked to leverage the casebased learning methodology by using videoconferencing (VC) technologies. Patient presentations/consultations are supplemented by "clinical updates", 15-20 minute didactic presentations focusing on evidence-based best practices and standard of care algorithms and procedures. Project ECHO started at the University of New Mexico as a novel way of treating rural patients with common, complex, and chronic conditions by leveraging the knowledge housed at Academic Medical Centers (AMCs). This methodology has been proven effective, not only as a system of disseminating clinical practice knowledge but also showing that increased education directly relates, and improves clinical outcomes in rural areas. [2, 3] In 2011, University of Utah Health Care's TeleHealth Services adopted and adapted the Project ECHO model.

Methods: Working in collaboration with the Internal Medicine and Pediatric (Med/Peds) residents, Project ECHO evolved as a medical residency educational tool, tailored specifically to their needs. This unique twist on the traditional Project ECHO methodology was facilitated, in part, due to the resources and amount of clinical specialties housed at the University of Utah. Based upon resident/faculty input we were able to facilitate sessions with leading experts in the fields of: hepatitis b, depression, heart failure/a. fib, hepatitis c, and rheumatoid arthritis. After these 60-90 minute case centered sessions, with a supplemental 'clinical update' on best practices, a survey is distributed to the residents/faculty to gauge program effectiveness. These surveys are analyzed to evaluate the significance and effectiveness of this new utilization of Project ECHO principles. This is an on-going program with data points being collected indefinitely into the future.

Results: 40 paired (pre/post) quantitative data points were captured after each Med/Peds Residency ECHO session which gaged the participants "knowledge" and "comfort" level in diagnosing and treating different clinical areas/disorders covered, mentioned above, by a traditional Likert scale: 1 (minimal/beginner) to 5 (extensive/expert). Cumulatively across all clinical areas the knowledge level pre-ECHO session mean score was a 2.875 , and post-ECHO session score was 3.625. This increase was extremely statitistically significant with a two-tailed pvalue $<0.001$ as measured by a paired two sample for means, t-test. Likewise, data was also collected concerning the comfort level with diagnosis and treating a clinical area/disorder. Mean pre-ECHO session score was 2.70 on the Likert Scale and the mean post-ECHO session increased to 3.55. This increase is also extremely statistically significant with a p-value measured at $<0.001$. Beyond statistical results, much can be interpreted by the qualitative responses given by residents. A $3^{\text {rd }}$ year resident after a Cardiology session stated, "Excellent presentation and great learning points. Very valuable use of time." Additionally, after the Hepatitis C session a $4^{\text {th }}$ year resident simply stated, "I thought it was perfect. I really felt I learned a lot."

Conclusions: Integrating the Project ECHO methodology into a residency training program at an Academic Medical Center can improve the knowledge and comfort level concerning the "diagnosis and treatment" across multiple clinical areas. Further, allowing residents to pick what clinical areas they are interested in and/or need further education creates a tailored educational offering directly reflecting the needs of a specific population instead of general, scheduled, and wide-ranging education generally incorporated into medical residencies. This system of dynamic curriculum development is more flexible to the patient populations that these residents see in the community clinics around the Salt Lake Valley.

1. Strobel J, van Barneveld A. When is PBL more effective? A metasynthesis of meta-analyses comparing PBL to conventional classrooms. Interdisc J Problem-based Learn 2009; 3(1):4.

2. Arora S, Thornton K, Murata G, Deming P, Kalishman S, Dion D, et al. Outcomes of treatment for hepatitis $\mathrm{C}$ virus infection by primary care providers. N Engl J Med 2011; 364(23):2199-2207.

3. Mitruka K, Thornton K, Cusick S, Orme C, Moore A, Manch RA, et al. Expanding primary care capacity to treat hepatitis $\mathrm{C}$ virus infection through an evidence-based care model: Arizona and Utah, 2012-2014. MMWR Morb Mortal Wkly 2014; 63(18):393-98.

\section{Objectives}

1. Interpret the results of the statistical analysis and its application in graduate medical education

2. Conclude that the results have practical applications

3. Critique and provide feedback about study, and future study design

\section{SUNDAY, MAY 15, 2016}

5:35 PM-6:00 PM Sunday, May 15, 2016

\section{EPOSTERS: MEET THE AUTHORS IN THE ATA EXPERIENCE ZONE/EXHIBIT HALL}

\section{P13 THE IMPORTANCE OF CULTURAL INFUSION IN DESIGNING A TELEMEDICINE-BASED HEALTH CENTER \\ PRESENTER: \\ Shelley Cooper, BSBA, MAT, EDD (Candidate) \\ Nova Southeastern University, Kansas City, KS}

Acknowledgement and appreciation of a patient's culture is vital to the acceptance of healthcare by individuals from different backgrounds. When attempting to treat patients, it is critical to become aware and respectful of differences in values, beliefs and cultural heritages. Galanti (2004) cited several instances where awareness, acknowledgment and acceptance of differing cultural profiles were imperative to the physiological well-being of patients in their medical treatment and procedures.

Several areas of cultural variances affect the patient's acceptance of medical treatment, especially in the use of telemedicine when the caregiver and patient are not occupying the same physical space. Cultures, subcultures, stereotypes and generalizations affect a patient's reactions to birth, pain, and end of life situations. As telemedicine becomes more prevalent, caregivers and other participants operating within the field of healthcare must consider several topics when they relate to interactions with patients. Language, religion, communication style, time orientation, non-verbal communication, 
pain medication, sex roles and mental health play important roles in treating patients (Galanti, 2004). Consequently, the establishment of health centers within our communities and other settings such as schools will fare much better when these factors are infused into the design, construction, operation and management of these centers.

In addition, healthcare education that ignores the influence of culturebased models has been proven to be less effective in the learning process. Quite simply, "culture is critical to design and instruction" (Young, 2009, p. x). Keeping culture in mind when designing any type of interaction with diverse individuals can help caregivers understand how to treat a variety of patients.

Performing a needs assessment before designing the telemedicine program is critical to measuring the success of the program. Acknowledging the cultural differences between diverse populations as telemedicine programs are designed will help provide a more accepting environment for all participants. Several other steps are critical to the evaluation of success of the telemedicine program. Developers should acknowledge and infuse the following topics: values, cultures and subcultures, ethnicity, language, religious/spiritual influence and end of life. A pre- and post-questionnaire that inquires about the level of comfort for all patients will help identify and measure areas that need to be changed in order to create a more inclusive environment for all patients, regardless of their background.

1. Galanti GA. (2004) Caring for patients from different cultures, 3rd edition, University of Pennsylvania Press, Pennsylvania, PA.

2. Young PA. (2009) Instructional design frameworks and intercultural models. Information Science Reference, Hershey, NY.

\section{Objectives}

1. Provide an appreciation of cultural influences on telemedicine acceptance within diverse communities

2. Apply cultural differences to the design of telemedicine-based health care centers

3. Identify key areas where caregivers can acknowledge cultural differences among patients

\section{Track: Policy and Legal}

\section{SUNDAY, MAY 15, 2016}

5:35 PM-6:00 PM Sunday, May 15, 2016

\section{EPOSTERS: MEET THE AUTHOR IN THE ATA EXPERIENCE ZONE/EXHIBIT HALL}

\section{P14 BENEFITS AND BARRIERS TO CREDENTIALING BY PROXY \\ PRESENTER:}

Annalise F. Baker-Whitcomb, Program Coordinator

Medical University of South Carolina, Charleston, SC

Introduction: Telemedicine has quickly revolutionized health care, but is often slowed by key administrative barriers such as physician credentialing. The demands of physician credentialing can quickly become excessive when credentialing a large telemedicine roster at every site where the providers give consults. To address this administrative barrier, the Centers for Medicare and Medicaid Services instituted Credentialing by Proxy in 2011, a streamlined version of credentialing where the distant site hospital can act as the credentialing proxy for the originating site hospital. Originating site hospitals are required to maintain only limited documentation and are notified by the Proxy institution if any change in medical staff status is necessary. This process eliminates the repetition of these time intensive procedures and relieves significant administrative strain on the site hospitals and telemedicine program administrators, allowing higher efficiency and accelerated implementation. While this process is used extensively throughout the nation, a significant number of hospitals still opt for traditional credentialing of telemedicine providers. This project seeks to understand the barriers to implementing credentialing by proxy through the attitudes of relevant staff members within originating site hospitals.

Methods: This study utilized stratified purposeful sampling to recruit participants who manage credentialing at telemedicine site hospitals. Qualitative interviews were conducted, recorded and transcribed for accuracy in analysis. A semi-structured interview guide was utilized by the researcher to direct the conversation to the relevant subject matter. Data analysis was continuous, employing the constant comparative method to determine common themes. Results: The majority of study participants experienced uncertainty through a loss of control over the credentialing process for telemedicine providers. Participants often feared that the ultimate responsibility of any mistakes in this process would still fall back on them and their institution. Despite their uncertainty, many participants acknowledged the benefits and necessity of delegated credentialing when having to credential a large roster of telemedicine providers at a smaller institution. Certain factors were helpful in reducing concerns, including the awareness that the proxy institution is held by the same regulatory standards as the site hospital. Many of the site hospitals participating in delegated credentialing also mitigated their uncertainty by continuing to check certain requirements themselves, helping staff members to feel more confident in the qualifications of the providers. Many site hospitals also adopted a "wait and see approach" where they understood delegated credentialing to be in trial period at their institution, from which they could revert back if anything negative occurred.

Conclusions: This study resulted in several key findings that provide insight on reducing uncertainty regarding delegated credentialing. Assessing these concerns assists greatly in addressing them and overcoming the barriers to implementation of Credentialing by Proxy. The primary source of uncertainty for this sample was a loss of control over the process, which led to feelings of insecurity and uneasiness regarding the credentials of the telemedicine providers. This uncertainty could be reduced through several key strategies that increase the transparency and clarity of regulatory and hospital requirements for delegated credentialing.

1. Dresevic A, Kalmowitz CF. (2011). CMS Issues Final Rule on Credentialing and Privileging for Telemedicine. Regulatory Review, The Health Law Partners. Retrieved from http://www.thehealthlawpartners.com/files/ june_regulatoryreview.pdf

\section{Objectives}

1. Describes the challenges and delays of traditional credentialing for telemedicine providers and administrative staff

2. Identify key attitudes toward delegated credentialing at telemedicine sites

3. Apply tactics to reduce uncertainty for medical staff office members at telemedicine site hospitals

P15 ID PROOFING AND VERIFICATION USING WEB CAM, GESTURE BIOMETRICS AND PROFESSIONAL WITNESSES

\section{PRESENTER:}

Jeffrey Maynard, BSc, Chief Executive Officer

Biometric Signature ID, Lewisville, TX

The hypothesis is that reimbursement for telehealth services will be delayed as long as fraud is not reduced or eliminated. One of the best ways to reduce fraud is by effective identity proofing and verification that the patient about to receive services is the same one who initially registered. If we can develop a way to seamlessly address identity fraud we can provide more telehealth services and positively reduce morbidity and costs in the entire healthcare system. 


\section{ePOSTER PRESENTATIONS ABSTRACTS}

This presentation will outline how we can use a combination of "Witnesses" and software biometrics to provide an identity management strategy that can permit replacing a physical encounter with a live remote encounter. Ability to confirm that the patient ID is verified will help comply with emerging security and reimbursement issues. Fraud will become a large issue holding back telehealth services and reimbursements. It will be demonstrated that the process of ensuring that services provided are received by the rightful person can be performed and taped using the latest technology with trained authentication agents and using one or more software only biometrics. Reimbursement of the services will be more readily allowed since it is not subject to fraud. Participants will be exposed to the new emerging ANSI standards for Identity Proofing and Verification by North American Security Products Organization (NASPO). NASPO was chosen by the federal government to work with NIST and 32 other agencies and stakeholders to provide these new standards. The presenter is a committee member and a subject matter expert in this area with a biometrics company that has users in 70 countries.

The participants will be exposed to the new Identity Assurance levels 1-4 that will become the new ANSI standards for in person and remote identity proofing and verification. They will come away with an understanding of the technologies that are available to meet the federal standards. Recent data breaches will be addressed and their impact to the provider and the covered entities will be highlighted.

In his decree announced October 17, 2014 President Obama announced that use of Multi Factor Authentication is required for all agencies within 18 months. This decree will protect financial security fraud and reduce identity theft. The hypothesis is that the same regulations will be adapted by healthcare entities. Stakeholders should be made aware of the need for enhanced security and its availability through telemedicine.

1. IDPV Standard 6.5 - 2015 Requirements and Implementation Guidelines for Assertion, Resolution, Evidence, and Verification of Personal Identity Document No. NASPO-IDPV-090 September 10, 2015.

\section{Objectives}

1. Demonstrate awareness of new federal and regulatory identity proofing and verification strategies

2. Apply their new knowledge to determine the optimum ways they can increase reimbursements by offering identity verification

3. Identify differences in specific uses cases for biometric technologies 